Nevada

Environmental

Restoration

Project

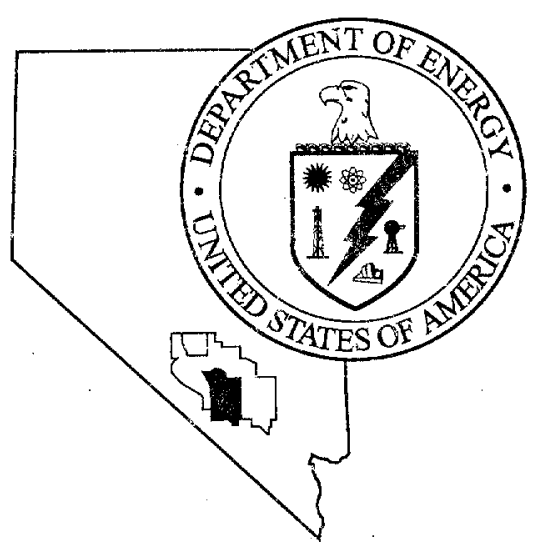

Closure Report for

Corrective Action Unit 340:

NTS Pesticide Release Sites

Nevada Test Site, Nevada

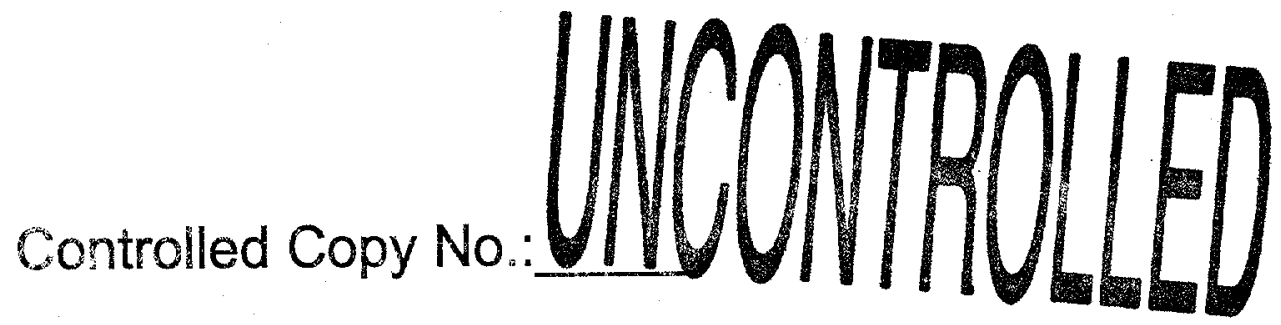

Revision: 0

May 2000

Environmental Restoration

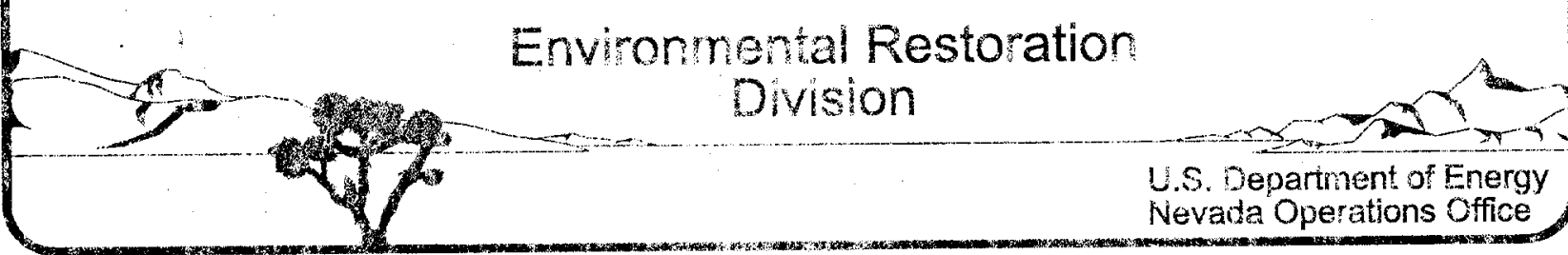




\section{DISCLAIMER STATEMENT}

Reference herein to any specific commercial product, process, or service by trade name, trademark, manufacturer, or otherwise, does not necessarily constitute or imply its endorsement, recommendation, or favoring by the U.S. Government or any agency thereof or its contractors or subcontractors.

\section{AVAILABILITY STATEMENT}

Available for sale to the public from:

U.S. Department of Commerce

National Technical Information Service

5285 Port Royal Road

Springfield, VA 22161-0002

Telephone: 800-553-6847 or 703-605-6000

Fax: 703-605-6900

E-mail: orders@ntis.fedworld.gov

Online ordering: http://www.ntis.gov/ordering.htm

Available electronically at http://www.doe.gov/bridge

Available for a processing fee to U.S. Department of Energy and its contractors, in paper, from:

U.S. Department of Energy

Office of Scientific and Technical Information

P.O. Box 62

Oak Ridge, TN 37831-0062

Telephone: $865-576-8401$

Fax: 865-576-5728

E-mail: reports@adonis.osti.gov 


\title{
CLOSURE REPORT FOR CORRECTIVE ACTION UNIT 340: NTS PESTICIDE RELEASE SITES, NEVADA TEST SITE, NEVADA
}

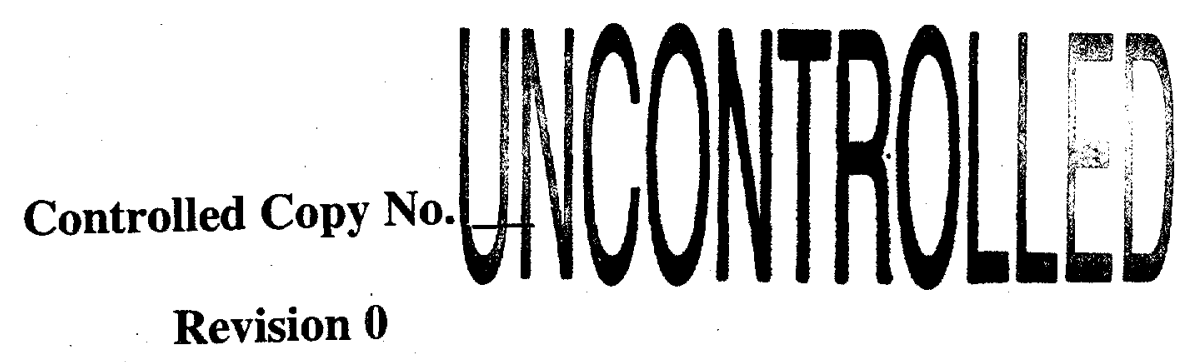

May 2000

\author{
Prepared for the U.S. Department of Energy \\ Nevada Operations Office \\ under Contract No. DE-AC08-96NV11718
}



DOE/NV-615

\section{CLOSURE REPORT FOR CORRECTIVE ACTION UNIT 340: NTS PESTICIDE RELEASE SITES, NEVADA TEST SITE, NEVADA}

Approved by: $\frac{\text { Jause }}{\text { Jandet L. Appenzeffer-Wing, Project Mahager }}$

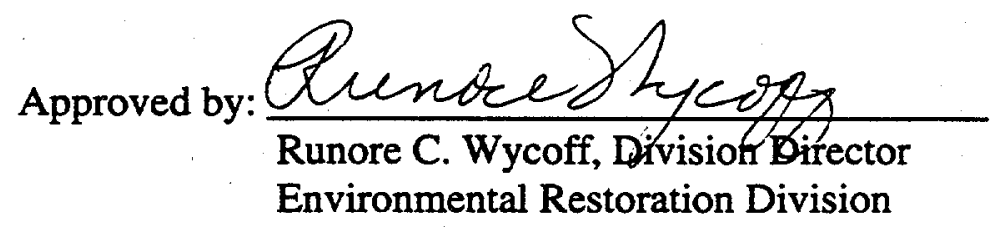
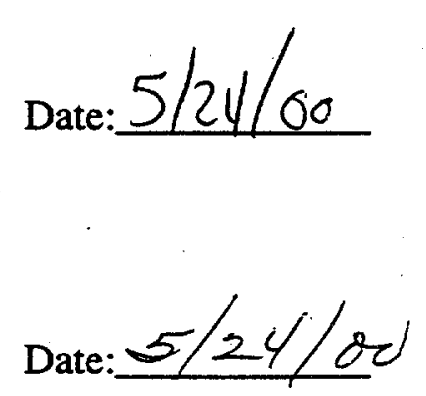



\section{TABLE OF CONTENTS}

ACRONYMS AND ABBREVIATIONS $\ldots \ldots \ldots \ldots \ldots \ldots \ldots \ldots \ldots \ldots \ldots$ vii

EXECUTIVE SUMMARY $\ldots \ldots \ldots \ldots \ldots \ldots \ldots \ldots \ldots \ldots \ldots \ldots \ldots \ldots \ldots$ ix

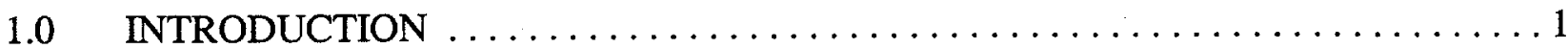

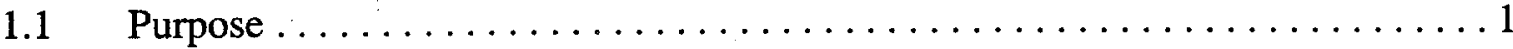

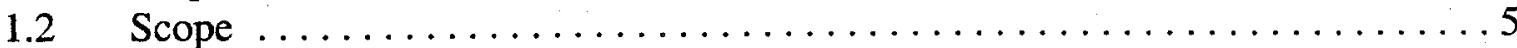

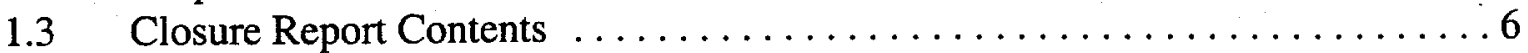

$2.0 \quad$ CLOSURE ACTIVITIES . . . . . . . . . . . . . . . . . . . . . . . . 9

2.1 Description of Corrective Action Activities $\ldots \ldots \ldots \ldots \ldots \ldots \ldots \ldots . \ldots$

2.1.1 Preplanning and Site Preparation ........................ 9

2.1.2 Excavation of Hazardous-Chlordane Soil . . . . . . . . . . . . . 9

2.1.3 Excavation of Petroleum Hydrocarbon Soil $\ldots \ldots \ldots \ldots \ldots \ldots \ldots 11$

2.1.4 Excavation of Nonhazardous Pesticide Soil $\ldots \ldots \ldots \ldots \ldots \ldots \ldots \ldots$

2.1.5 Disposal of Other Wastes . . . . . . . . . . . . . . . . . . . 13

2.1.6 Backfilling and Regrading of Excavations $\ldots \ldots \ldots \ldots \ldots \ldots \ldots \ldots$

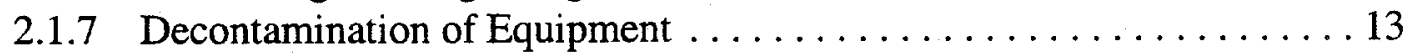

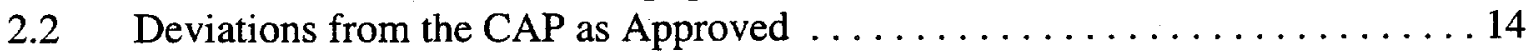

2.3 Corrective Action Schedule as Completed $\ldots \ldots \ldots \ldots \ldots \ldots \ldots \ldots \ldots$

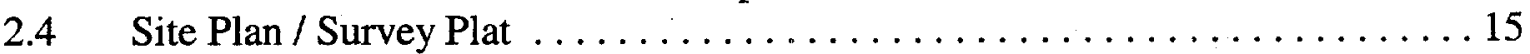

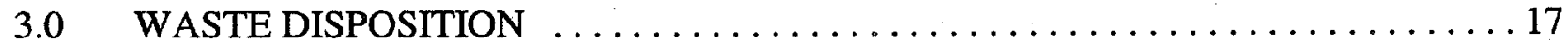

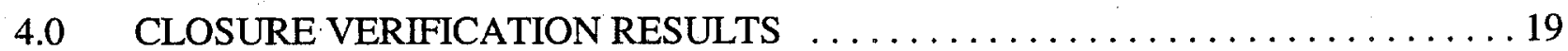

$4.1 \quad$ Verification Sample Analyses . . . . . . . . . . . . . . . . . . . . 19

4.2 Backfilling and Regrading of Excavations $\ldots \ldots \ldots \ldots \ldots \ldots \ldots$

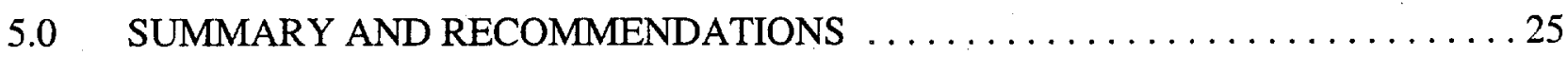

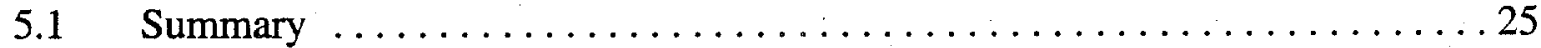

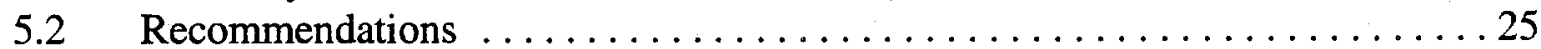

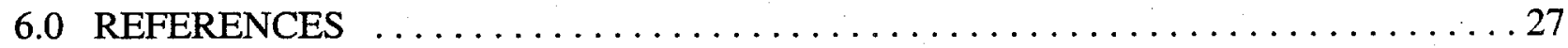




\section{TABLE OF CONTENTS (continued)}

\section{APPENDICES}

Appendix A: Photographs of Closure Activity Work Areas

Appendix B: Waste Disposition Documentation

Appendix C: Verification Sample Analytical Reports

Distribution List

NDEP Document Review Sheet

\section{FIGURES}

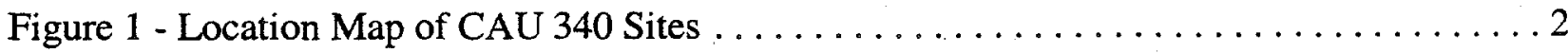

Figure 2 - Vicinity Map of CAU 340 Area 23 Sites $\ldots \ldots \ldots \ldots \ldots \ldots \ldots \ldots \ldots \ldots \ldots \ldots \ldots \ldots \ldots$

Figure 3 - Site Plan of CAU 340 Area 23 Sites Showing Areas and Depths of Excavations . . . 4

Figure 4 - Site Plan of CAU 340 Area 23 Sites Showing Verification Soil Sample Locations . 10

\section{TABLES}

Table 1 - CAU 340 Corrective Action Schedule as Completed . . . . . . . . . . . . . 16

Table 2 - Summary of Analytical Results for Detected Pesticides in Verification Samples ... . 20

Table 3 - Summary of Analytical Results for TPH in Verification Samples . . . . . . . . . 23 


\section{ACRONYMS AND ABBREVIATIONS}

\begin{tabular}{|c|c|}
\hline bgs & below ground surface \\
\hline $\mathrm{BN}$ & Bechtel Nevada \\
\hline CADD & Corrective Action Decision Document \\
\hline CAP & Corrective Action Plan \\
\hline CAS & Corrective Action Site \\
\hline $\mathrm{CAU}$ & Corrective Action Unit \\
\hline CR & Closure Report \\
\hline DOE & U.S. Department of Energy \\
\hline EPA & U.S. Environmental Protection Agency \\
\hline FFACO & Federal Facility Agreement and Consent Order \\
\hline $\mathrm{ft}$ & foot \\
\hline gal & gallon \\
\hline $\mathrm{L}$ & liter \\
\hline $\mathrm{m}$ & meter \\
\hline $\mathrm{m}^{3}$ & cubic meter \\
\hline $\mathrm{mg} / \mathrm{kg}$ & milligram per kilogram \\
\hline $\mathrm{mg} / \mathrm{L}$ & milligram per liter \\
\hline
\end{tabular}




\section{ACRONYMS AND ABBREVIATIONS (continued)}

NDEP

Nevada Division of Environmental Protection

NTS

Nevada Test Site

PRG

Preliminary Remediation Goal

TCLP

Toxicity Characteristic Leachate Procedure

TSDF

Treatment, Storage, and Disposal Facility

$\mathrm{ug} / \mathrm{kg}$

microgram per kilogram

$\mathrm{ug} / \mathrm{L}$

microgram per liter

$\mathrm{yd}^{3}$

cubic yard 


\section{EXECUTIVE SUMMARY}

The NTS Pesticide Release Sites are identified in the Federal Facility Agreement and Consent Order as Corrective Action Unit (CAU) 340. CAU 340 consists of the following three Corrective Action Sites (CASs) located in Areas 15 and 23 of the Nevada Test Site (NTS):

- CAS 15-18-02: Area 15 Quonset Hut 15-11 Pesticide Storage

- CAS 23-21-01: Area 23 Quonset Hut 800 Pesticide Release Ditch

- $\quad$ CAS 23-28-03: Area 23 Skid Huts Pesticide Storage

The Area 15 Quonset Hut 15-11 was formerly used for pesticide storage. The site characterization investigation did not find pesticides above U.S. Environmental Protection Agency (EPA) Industrial Preliminary Remediation Goals (PRGs) so corrective action was not required. EPA Industrial PRGs for pesticides and the Nevada Division of Environmental Protection (NDEP) Action Level for petroleum hydrocarbons were exceeded at the two CAU 340 sites in Area 23. The Area 23 Quonset Hut 800 was formerly used to steam-clean pesticide and herbicide equipment. The rinsate occasionally overflowed a sump into drainage ditches that ran south and southeast from the quonset hut. The Area 23 Skid Huts were formerly used for storing and mixing pesticide and herbicide solutions. Excess solutions were released directly to the ground near the skid huts. Clean-closure corrective actions were required for these two CASs in Area 23.

Based on analytical results reported in the site characterization report, soil in three broad areas at the Area 23 sites exceeded EPA Industrial PRGs for pesticides. Within these areas, soil in one location also exceeded the EPA hazardous waste level for pesticide toxicity. Soil in that location, and in two other locations, exceeded the NDEP Action Level for petroleum hydrocarbons. Finally, site debris, including remaining skid huts, needed to be removed and properly disposed.

The clean-closure corrective action consisted of excavation, disposal, verification sampling, partial backfilling, and regrading. Field activities began on November 3, 1999, and ended on January 13, 2000. Soil that exceeded the EPA hazardous waste level was excavated, staged in a covered rolloff bin within a 90-Day Pad, and hauled off-site for disposal. Soil that exceeded EPA Industrial PRGs for pesticides and the NDEP Action Level for petroleum hydrocarbons was excavated and disposed, along with the remaining skid huts and other site debris, in appropriate landfills at the NTS. Verification soil samples were collected and analyzed to determine if clean closure had been achieved. Some areas required additional excavation before resampling verified clean closure. Clean borrow soil was hauled to the site and used to backfill selected excavations. All excavations were then regraded to promote drainage and minimize ponding of surface water. 



\subsection{INTRODUCTION}

This Closure Report (CR) describes the remediation activities performed at the Nevada Test Site (NTS) Pesticide Release Sites where pesticides and herbicides were formerly stored, handled, and disposed. The NTS Pesticide Release Sites are identified in the Federal Facility Agreement and Consent Order (FFACO) of 1966 as Corrective Action Unit (CAU) 340 (FFACO, 1996). Remediation of CAU 340 is required under the FFACO (FFACO, 1996). CAU 340 consists of the following Corrective Action Sites (CASs) in Areas 15 and 23 of the NTS (Figure 1):

- CAS 15-18-02: Area 15 Quonset Hut 15-11 Pesticide Storage

- CAS 23-21-01: Area 23 Quonset Hut 800 Pesticide Release Ditch

- CAS 23-28-03: Area 23 Skid Huts Pesticide Storage

\subsection{PURPOSE}

The purpose of this report is to provide documentation of the completed corrective action and to provide data confirming the corrective action. The corrective action was performed in accordance with the approved Corrective Action Plan (CAP) (U.S. Department of Energy [DOE], 1999) and consisted of clean closure by excavation and disposal.

The Area 15 Quonset Hut 15-11 was formerly used for storage of farm supplies including pesticides, herbicides, and fertilizers. The Area 23 Quonset Hut 800 was formerly used to clean pesticide and herbicide equipment. Steam-cleaning rinsate and sink drainage occasionally overflowed a sump into adjoining drainage ditches. One ditch flows south and is referred to as the quonset hut ditch. The other ditch flows southeast and is referred to as the inner drainage ditch. The Area 23 Skid Huts were formerly used for storing and mixing pesticide and herbicide solutions. Excess solutions were released directly to the ground near the skid huts. The skid huts were moved to a nearby location prior to the site characterization performed in 1998 and reported in the Corrective Action Decision Document (CADD) (DOE, 1998). The vicinity and site plans of the Area 23 sites are shown in Figures 2 and 3, respectively.

The CADD (DOE, 1998) reported that at the Area 15 site, soil pesticide concentrations did not exceed U.S. Environmental Protection Agency (EPA) Region 9 Industrial Preliminary Remediation Goals (PRGs) to a depth of 0.3 meters $(\mathrm{m})$ (1 foot [ft]) below ground surface (bgs) (EPA, 1996a). Corrective actions at the Area 15 site were therefore not required. 


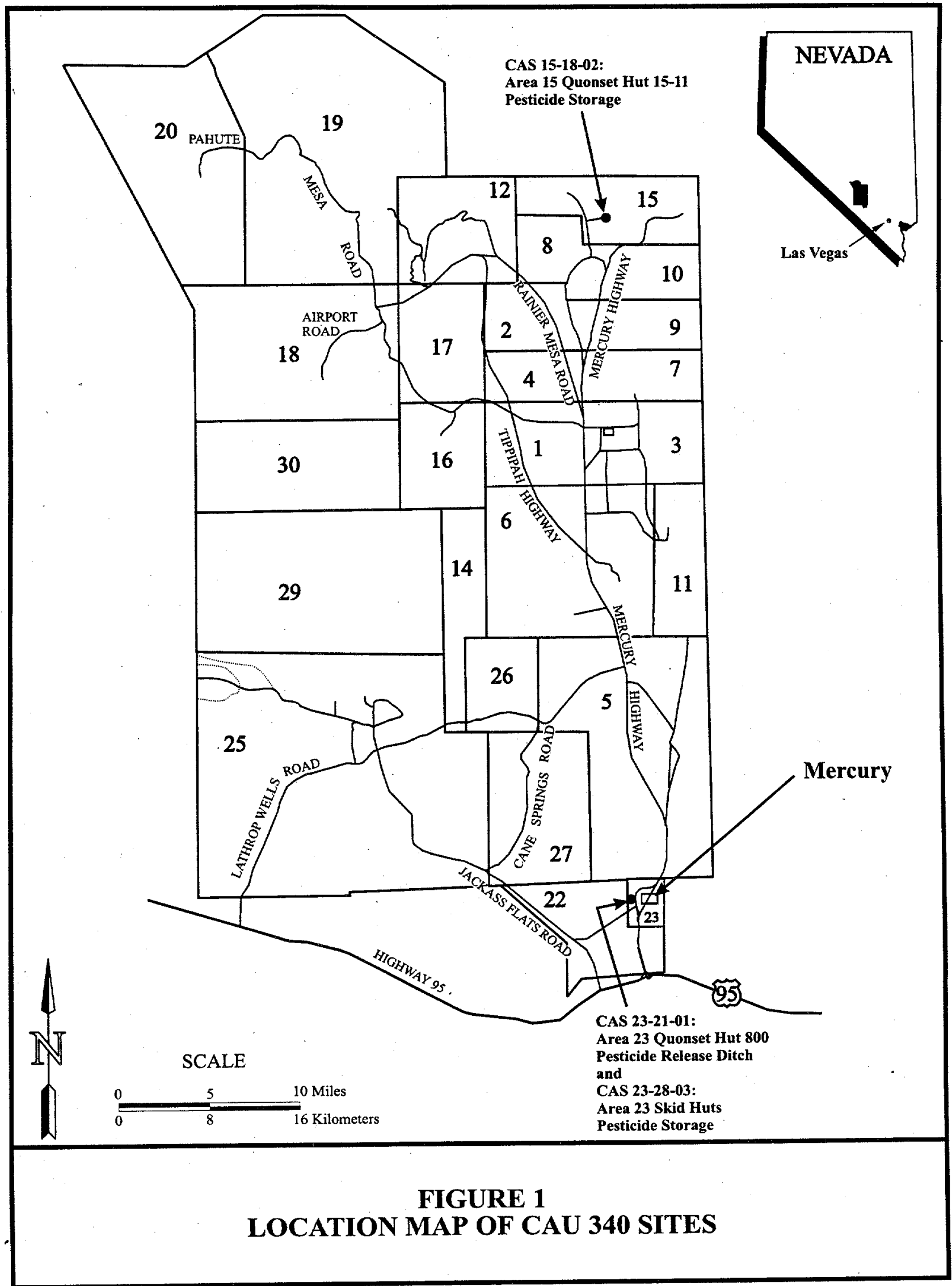




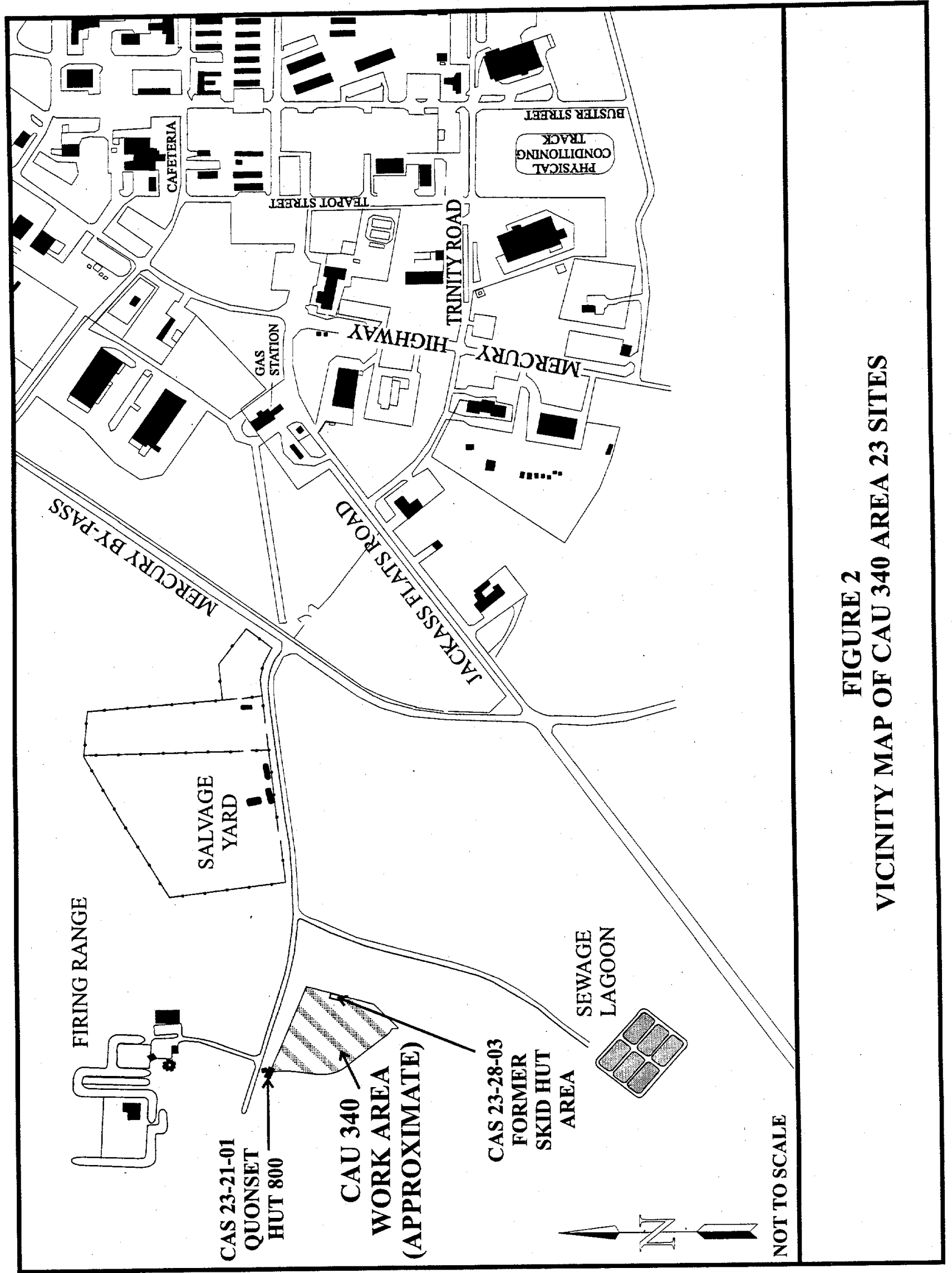




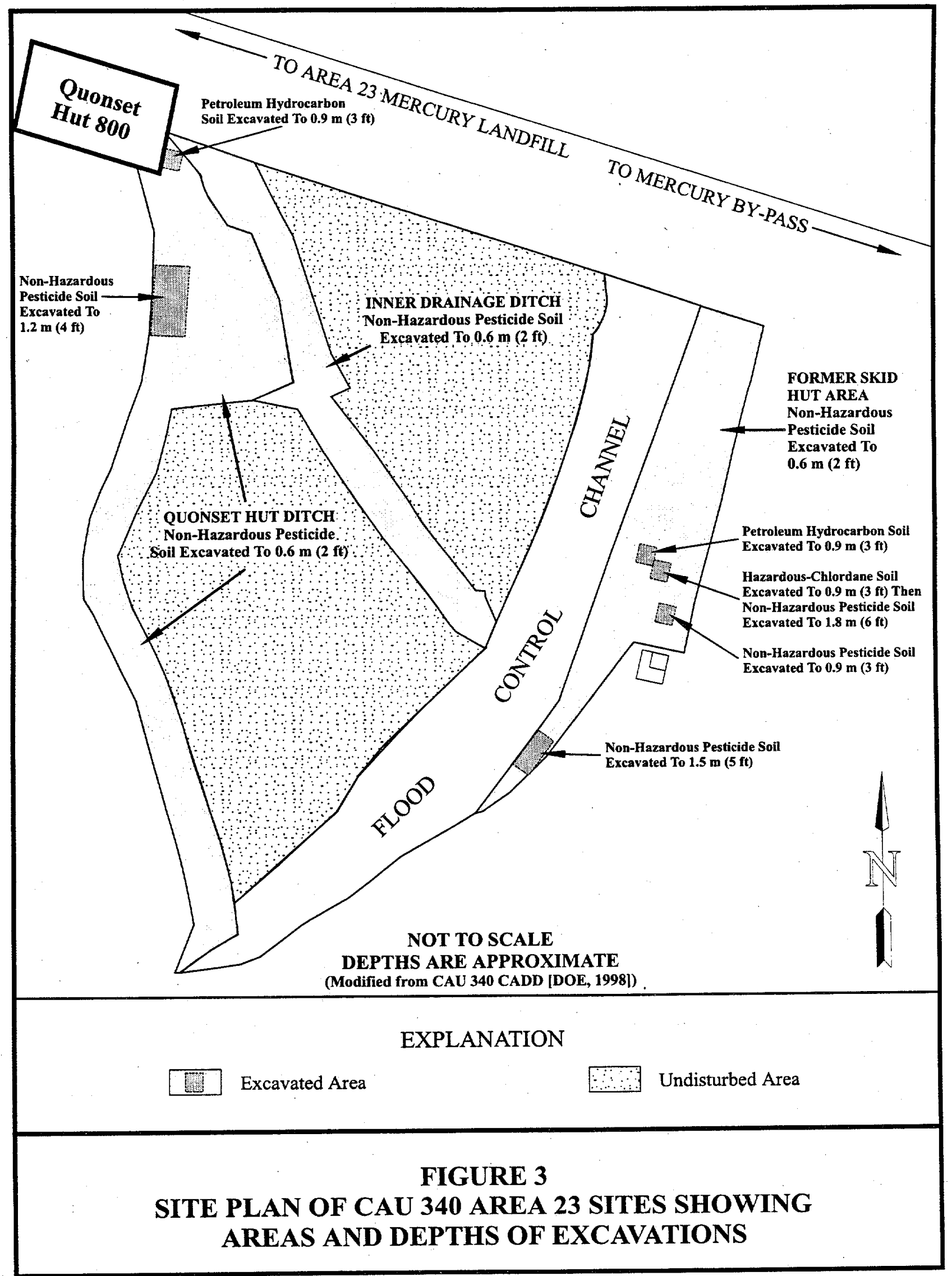


The CADD (DOE, 1998) reported that at the Area 23 sites (Quonset Hut 800 ditches and former skid hut area), soil exceeded EPA Industrial PRGs (EPA, 1996a) between the depths of 0 and 0:6 $\mathrm{m}(0$ and $2 \mathrm{ft})$ for the following pesticides:
- 4,4'-Dichloro Diphenyl Dichloro Ethane
- $\quad$ Aldrin
- 4,4'-Dichloro Diphenyl Dichloro Ethylene
- Dieldrin
- 4,4'-Dichloro Diphenyl Trichloro Ethane
- Heptachlor
- Chlordane
- Heptachlor Epoxide

Soil in one location in the former skid hut area exceeded the EPA Toxicity Characteristic Leachate Procedure (TCLP) hazardous-waste concentration for the pesticide Chlordane (EPA, 1996c). The soil from this location and in two other locations exceeded the Nevada Division of Environmental Protection (NDEP) Action Level for petroleum hydrocarbons (Nevada Administrative Code, 1996). Corrective actions at the Area 23 sites were therefore required.

The NTS Resource Management Plan Annual Summary (DOE, 2000) reiterated that characterization and remediation of FFACO sites are performed to industrial standards, which restricts future use of these sites to industrial use. Therefore, formal land-use restrictions are not necessary for sites that meet industrial standards.

\subsection{SCOPE}

The corrective action as implemented consisted of the following activities:

- Preplanning and site preparation, including preparation of plans and permits, delineation of excavation boundaries, and mobilization of equipment and personnel to the site.

- Excavation of soil exceeding the TCLP hazardous waste level for the pesticide Chlordane. The excavated soil was staged in a temporary 90 -day hazardous waste area and then transported to an off-site treatment, storage, and disposal facility (TSDF).

- Excavation of soil exceeding the NDEP Action Level for petroleum hydrocarbons. This soil was transported to the NTS Area 6 Hydrocarbon Class III Industrial Solid Waste Disposal Site (commonly referred to as the Hydrocarbon Landfill).

- $\quad$ Excavation of soil exceeding pesticide PRGs. This soil was transported to the NTS Area 23 Class II Municipal and Industrial Solid Waste Disposal Site (commonly referred to as the Mercury Landfill) for disposal. 
- Visually inspecting excavations, field screening samples, and collecting verification soil samples for laboratory analysis. Based on results of field screening and verification samples, additional excavation was done in certain areas and resampled.

- Transporting clean borrow soil to the site for backfilling.

- $\quad$ Regrading the excavations to promote drainage and minimize ponding of surface water.

- Site cleanup, including disposal of remaining skid huts, fencing, and other site debris in the NTS Area 9 U10c Class III Industrial Solid Waste Disposal Site (commonly referred to as the U10c Landfill).

\subsection{CLOSURE REPORT CONTENTS}

This document is divided into the following sections in accordance with the approved FFACO CR standardized outline:

- Section 1.0 - Introduction (purpose, scope, contents).

- Section 2.0 - Closure Activities (description, deviations, schedule, site plan).

- Section 3.0 - Waste Disposition (wastes encountered and their appropriate disposal).

- $\quad$ Section 4.0 - Closure Verification Results (laboratory analysis).

- Section 5.0 - Summary and Recommendations.

- Section 6.0 - References.

Certain sections and appendices of this document have been modified from the approved FFACO outline. The following FFACO sections and appendices have not been included or revised as indicated below:

- Use Restriction - Not applicable. Clean closure of FFACO sites to industrial standards restricts future use to industrial use (DOE, 2000).

- Closure Certification - Not applicable.

- As-Built Documentation - Not applicable. No engineered structures were constructed.

- Modifications to the Post-Closure Plan - Not applicable for this clean close site. 
The appendices included in this document are provided as follows:

- Appendix A: Photographs of Closure Activity Work Areas.

- Appendix B: Waste Disposition Documentation.

- Appendix C: Verification Sample Analytical Reports. 



\subsection{CLOSURE ACTIVITIES}

This section of the CR details the specific corrective action activities implemented and completed during the closure of CAU 340. This section also provides a detailed schedule of site activities as completed. Photographs showing the work areas before, during, and after closure activities are included in Appendix A.

\subsection{DESCRIPTION OF CORRECTIVE ACTION ACTIVITIES}

\subsubsection{Preplanning and Site Preparation}

Planning documents prepared prior to beginning CAU 340 corrective action activities include the CAP (DOE, 1999), Field Management Plan (Bechtel Nevada [BN], 1999a), Site Specific Health and Safety Plan (BN, 1999b), a construction work package, and an excavation permit. Aboveground and underground utilities were identified prior to starting work. Based on process knowledge and analytical results from the site characterization, a Radiological Control Technician exempted the waste loads from radiological survey. In addition, a National Environmental Policy Act checklist was prepared and a pre-activity site survey was performed by a biologist. The survey confirmed the absence of sensitive animal or plant species. Planned excavation boundaries were identified. A Readiness Review meeting was conducted on October 20,1999. On November 3,1999, the pre-job briefing was held and personnel and equipment were mobilized to the site.

\subsubsection{Excavation of Hazardous-Chlordane Soil}

The CADD (DOE, 1998) identified one location in the former skid hut area where soil exceeded the TCLP level for the pesticide Chlordane (Figure 3). This soil required removal as hazardous waste. A 3 by $3 \mathrm{~m}$ (10 by $10 \mathrm{ft}$ ) excavation was delineated, centered on the original sitecharacterization soil-sampling location.

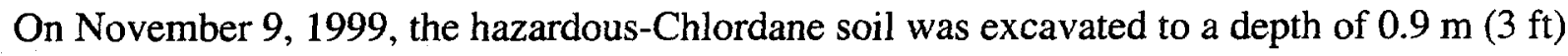
bgs, $0.3 \mathrm{~m}(1 \mathrm{ft})$ below the deepest sample reported in the CADD (DOE, 1998) to exceed the TCLP level. Using a backhoe, the soil was excavated and loaded directly into a 15 cubic meter $\left(\mathrm{m}^{3}\right)$ (20 cubic yard $\left.\left[\mathrm{yd}^{3}\right]\right)$ rolloff bin provided by the off-site TSDF subcontractor. The bin was covered with a tarp and staged in a 90-day hazardous waste area pending transport to an off-site TSDF. The completed excavation was visually inspected and no staining or discoloration was observed.

Soil samples collected in the former skid hut area are identified with a "SK" prefix. The samples were collected from the sides (SK01 through SK04) and bottom (SK05) of the hazardouschlordane excavation at locations shown in Figure 4. Splits of the soil samples were field 


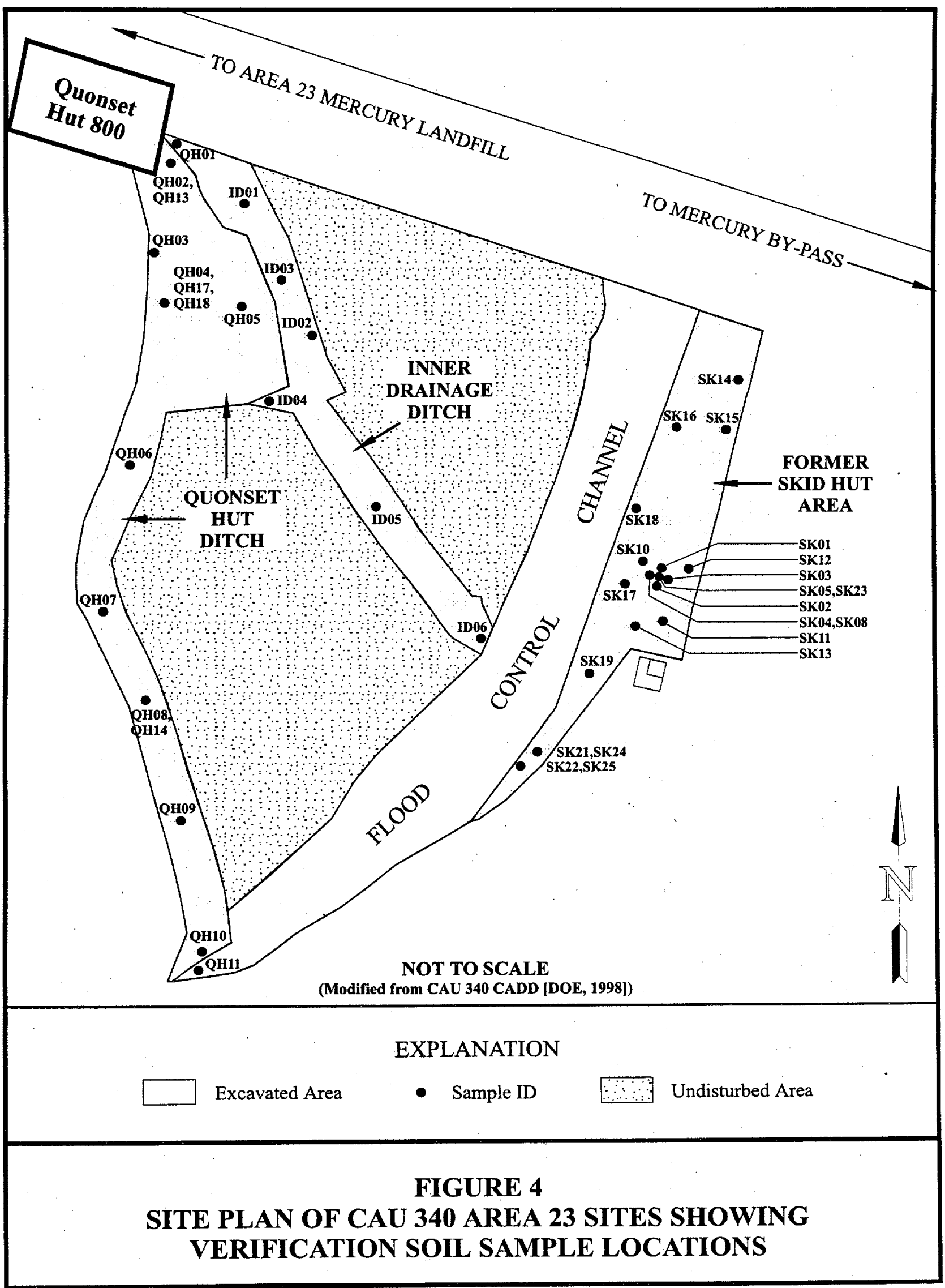


screened for pesticides and petroleum hydrocarbons using test kits but the results were inconclusive. The remaining sample portions were sent to an off-site laboratory for verification analyses. Details of soil sampling, handling, analyses, and results are discussed in Section 4.1.

The analytical results indicate that TCLP levels for pesticides and the NDEP Action Level for petroleum hydrocarbons are not exceeded. However, the bottom soil sample (SK05) exceeded

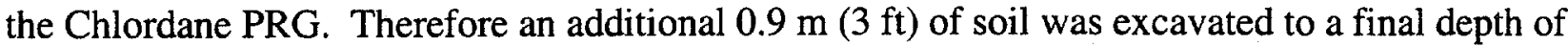
$1.8 \mathrm{~m}(6 \mathrm{ft}$ ) bgs on January 3, 2000. A final verification soil sample (SK23) was collected from the bottom of the deepened excavation (Figure 4). The analytical results are discussed in Section 4.1 and indicate that pesticide PRGs are not exceeded and no further excavation is needed.

On December 16, 1999, approximately $9 \mathrm{~m}^{3}\left(12 \mathrm{yd}^{3}\right)$ (16 tons) of hazardous-Chlordane soil was transported off-site for disposal by the TSDF subcontractor. On January 3, 2000, approximately $7 \mathrm{~m}^{3}$ ( $\left(9 \mathrm{yd}^{3}\right)$ (12 tons) of nonhazardous pesticide soil was disposed in the NTS Area 23 Mercury Landfill. Waste disposition is discussed in Section 3.0. Backfilling activities are discussed in Section 2.1.6.

\subsubsection{Excavation of Petroleum Hydrocarbon Soil}

The CADD (DOE, 1998) identified three locations where soil exceeded the petroleum hydrocarbon NDEP Action Level and pesticide PRGs. The first location was the hazardousChlordane soil described in the previous section. The second location was also in the former skid hut area, adjacent to the hazardous-Chlordane soi. The third location was in the quonset hut ditch. The second and third locations were delineated for excavation (Figure 3), each approximately 3 by $3 \mathrm{~m}(10$ by $10 \mathrm{ft})$ and centered on the original site-characterization soilsampling locations.

On November 8, 16, and 17, 1999, petroleum hydrocarbon soil was excavated to a depth of $0.9 \mathrm{~m}$ ( $3 \mathrm{ft}$ ) bgs using a backhoe and loaded into end dumps using a front-end loader. The completed excavations were visually inspected and no staining or discoloration was observed.

Soil samples collected in the former skid hut area and quonset hut ditch are identified with "SK" and "QH" prefixes, respectively. The samples were collected from the bottom of the two petroleum hydrocarbon excavations (SK10 and QH02/QH13, respectively) at locations shown in Figure 4. Splits of the soil samples were field screened for pesticides and petroleum hydrocarbons using test kits but the results were inconclusive. The remaining sample portions were sent to an off-site laboratory for verification analyses. Details of soil sampling, handling, analyses, and results are discussed in Section 4.1. The analytical results indicate that the NDEP Action Level and pesticide PRGs are not exceeded and no further excavation is needed. 
Approximately $41 \mathrm{~m}^{3}\left(53 \mathrm{yd}^{3}\right)$ (71 tons) of nonhazardous petroleum hydrocarbon soil were disposed in the NTS Area 6 Hydrocarbon Landfill. Waste disposition is discussed in Section 3.0. Backfilling activities are discussed in Section 2.1.6.

\subsubsection{Excavation of Nonhazardous Pesticide Soil}

The CADD (DOE, 1998) identified three broad areas where pesticide soil concentrations exceeded PRGs but not TCLP levels (Figure 3): former skid hut area, quonset hut ditch, and inner drainage ditch. Areas were delineated for excavation based on the original sitecharacterization soil-sampling locations.

On November 10-16, 1999, nonhazardous pesticide soil was excavated to a depth of $0.6 \mathrm{~m}(2 \mathrm{ft})$ bgs using a front-end loader and scraper. A small area, approximately 3 by $3 \mathrm{~m}$ ( 10 by $10 \mathrm{ft}$ ) and

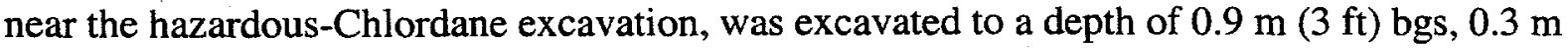
(1 ft) below a sample reported in the CADD (DOE, 1998) to exceed a PRG. The completed excavations were visually inspected and no staining or discoloration was observed.

Soil samples collected in the former skid hut area, quonset hut ditch, and inner drainage ditch are identified with an "SK," "QH," and " $\mathrm{D}$ " prefixes, respectively. Verification soil samples (SK11-SK22, QH01 and QH03-QH11, and ID01-ID06, respectively) were collected from the bottom of the excavations at locations shown in Figure 4. Details of soil sampling, handling, analyses, and results are discussed in Section 4.1. The analytical results indicate that pesticide PRGs are not exceeded except at locations QH04, SK21, and SK22, which exceeded the Chlordane PRG.

On January 3-4,2000, two locations, each approximately 3 by $9 \mathrm{~m}$ ( 10 by $30 \mathrm{ft}$ ) and centered on SK21/SK22 and QH04, were excavated deeper. The SK21/SK22 location was excavated an additional $0.9 \mathrm{~m}(3 \mathrm{ft})$ to a final depth of $1.5 \mathrm{~m}(5 \mathrm{ft}) \mathrm{bgs}$. The QH04 location was excavated an additional $0.6 \mathrm{~m}(2 \mathrm{ft})$ to a final depth of $1.2 \mathrm{~m}(4 \mathrm{ft})$ bgs. Soil samples were collected from the bottom of both excavations (SK24/SK25 and QH17/QH18[duplicate], respectively). Splits of the soil samples were field screened for pesticides and petroleum hydrocarbons using test kits but the results were inconclusive. The remaining sample portions were sent to an off-site laboratory for verification analyses. The analytical results are discussed in Section 4.1 and indicate that pesticide PRGs are not exceeded in the remaining soil and no further excavation is needed.

Approximately $1,700 \mathrm{~m}^{3}\left(2,200 \mathrm{yd}^{3}\right)$ (2,900 tons) of nonhazardous pesticide soil was disposed in the NTS Area 23 Mercury Landfill. Waste disposition is discussed in Section 3.0. Backfilling activities are discussed in Section 2.1.6. 


\subsubsection{Disposal of Other Wastes}

On January 13, 2000, two skid huts that had been relocated away from the excavation area and a pallet of debris were hauled to the NTS Area 9 U10c Landfill for disposal.

\subsubsection{Backfilling and Regrading of Excavations}

Prior to the start of field activities, a source of backfill material was identified and used to backfill the former skid hut area excavation and portions of the quonset hut ditch and inner drainage ditch excavations in order to restore drainage and minimize ponding of surface water.

Between November 3, 1999, and November 18, 1999, clean borrow soil was stockpiled on-site. The borrow soil was subsequently placed in the excavations and wheel compacted using the front-end loader, grader, or water truck, as needed. In small excavations with restricted access, the soil was compacted with downward blows of the backhoe bucket until wheel compaction was possible. Final site regrading was completed on January 13, 2000.

\subsubsection{Decontamination of Equipment}

After the final load of hazardous-Chlordane soil was handled, equipment that had contacted the soil (backhoe bucket and shovel) was cleaned by first brushing off visible residue, then washing with a laboratory-grade detergent solution, and followed by a tap water rinse. Equipment decontamination was performed over the rolloff bin in order to contain rinsate in the excavated soil. Less than 4 liters [L] (1 gallon [gal]) of detergent solution and water rinse was used and was completely absorbed into the waste soil.

Soil sampling scoops were decontaminated before and after for sample collection using a laboratory-grade detergent solution, an isopropanol rinse and deionized water rinses. Less than $4 \mathrm{~L}$ ( $1 \mathrm{gal}$ ) of detergent solution and rinsate was generated from the decontamination of scoops used to sample hazardous-Chlordane soil and was completely absorbed into soil in the rolloff bin. Less than $4 \mathrm{~L}(1 \mathrm{gal})$ of detergent solution and rinsate was generated from the decontamination of other scoops and was completely absorbed in soil later disposed in the NTS Area 23 Mercury Landfill. 


\subsection{DEVIATIONS FROM THE CAP AS APPROVED}

No significant deviations occurred from the approved scope of work as outlined in the CAP (DOE, 1999). The following minor deviations occurred from the approved scope of work:

- $\quad$ Some locations were excavated as much as $1.2 \mathrm{~m}(4 \mathrm{ft})$ deeper than the planned depths of 0.3 to $0.6 \mathrm{~m}$ ( 1 to $2 \mathrm{ft}$ ) bgs. The planned excavation depth at some locations was the same depth as the site-characterization soil samples that exceeded regulatory levels

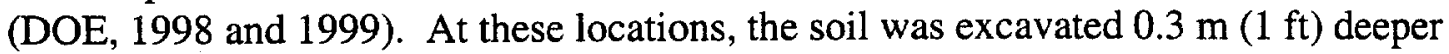
than planned to increase the likelihood that all the waste would be removed at one time. Despite these precautions, some soil samples did exceed the Chlordane EPA Industrial PRG and further excavation was necessary to remove all the waste. Those locations were excavated an additional 0.6 to $0.9 \mathrm{~m} \mathrm{(2} \mathrm{to} 3 \mathrm{ft}$ ) and then re-sampled.

- Verification soil samples were collected in four more locations than planned. Chlordane concentrations at locations QH04, SK05, SK21, and SK22, exceeded the EPA Industrial PRG and prompted further excavation and re-sampling of those locations at greater depths (QH17/QH18, SK23, SK24, and SK25, respectively).

- Actual volumes of generated waste varied from planned volumes. A slightly greater volume of hazardous-Chlordane soil was excavated and disposed $\left(9 \mathrm{~m}^{3}\left[12 \mathrm{yd}^{3}\right]\right)$ than planned $\left(7.5 \mathrm{~m}^{3}\left[10 \mathrm{yd}^{3}\right]\right)$ because that excavation was dug $0.3 \mathrm{~m}(1 \mathrm{ft})$ deeper than planned. The volume of nonhazardous petroleum-hydrocarbon soil was not estimated separately from nonhazardous pesticide soil in the plan but $41 \mathrm{~m}^{3}\left(53 \mathrm{yd}^{3}\right)$ were excavated and disposed. A smaller volume of nonhazardous pesticide soil was excavated and disposed $\left(1,700 \mathrm{~m}^{3}\left[2,200 \mathrm{yd}^{3}\right]\right)$ than planned $\left(2,300 \mathrm{~m}^{3}\left[3,000 \mathrm{yd}^{3}\right]\right)$ because the construction equipment excavated the ditches more efficiently than expected, concentrating the excavation along the deepest portion of the ditches where overflow from the quonset hut would have traveled.

- The former skid hut area excavation was completely backfilled with clean fill and leveled to existing grade, as planned. The quonset hut ditch and inner drainage ditch excavations were not fully backfilled, as had been planned. The ditches needed only partial backfilling before regrading to restore the drainages to more natural conditions.

- All nonhazardous and non-petroleum hydrocarbon waste was disposed in the NTS Area 23 Mercury Landfill, as planned, except for a load of two skid huts and a pallet of debris which was disposed in the NTS Area 9 U10c Landfill. 


\subsection{CORRECTIVE ACTION SCHEDULE AS COMPLETED}

The corrective action field activities began on November 3, 1999, and were completed on January 13, 2000. A corrective action schedule as completed is provided in Table 1.

\subsection{SITE PLAN / SURVEY PLAT}

The final site plan is shown in Figure 3. 
TABLE 1

CAU 340 CORRECTIVE ACTION SCHEDULE AS COMPLETED

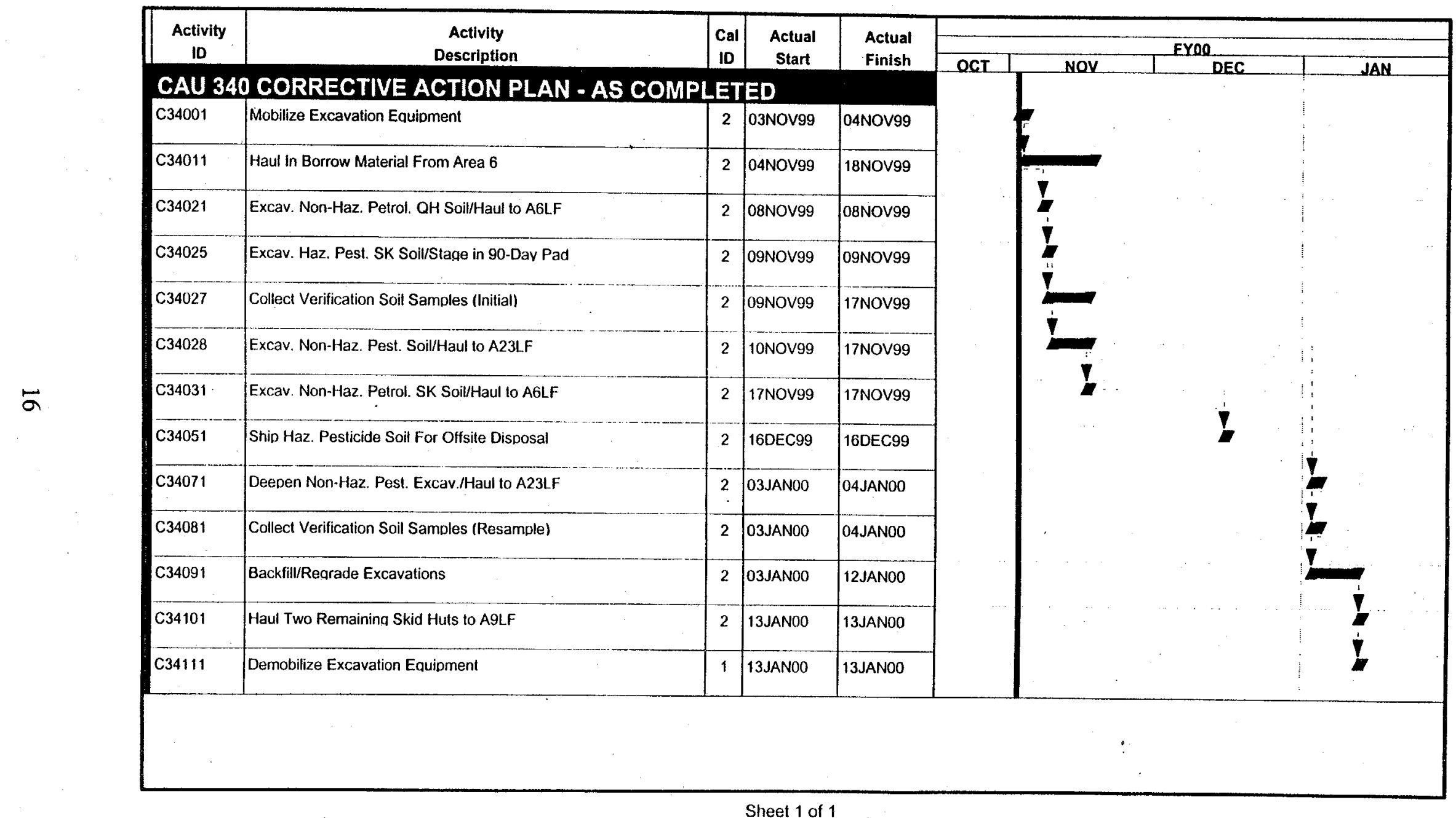




\subsection{WASTE DISPOSITION}

Wastes generated during the closure of CAU 340 were disposed as follows:

- Approximately $9 \mathrm{~m}^{3}\left(12 \mathrm{yd}^{3}\right)$ (16 tons) of hazardous-Chlordane soil was staged on-site in a 90-Day Pad (\#90D016) and then transported off-site as hazardous waste to the TSDF on December 16, 1999.

- $\quad$ Approximately $41 \mathrm{~m}^{3}\left(53 \mathrm{yd}^{3}\right)$ (71 tons) of nonhazardous petroleum-hydrocarbon soil was disposed in the NTS Area 6 Hydrocarbon Landfill on November 8, 16, and 17, 1999.

- Approximately $1,700 \mathrm{~m}^{3}\left(2,200 \mathrm{yd}^{3}\right)$ (2,900 tons) of nonhazardous pesticide soil was disposed in the NTS Area 23 Mercury Landfill on November 10-16, 1999, and on January 3-4, 2000.

- Approximately 21 tons of solid waste, including the two remaining skid huts, were disposed in the NTS Area 9 U10c Landfill on January 13, 2000.

- Potentially hazardous waste, consisting of spent carbon tetrachloride, hydrochloric acid, and methanol, was generated during field screening of soil samples. This waste was managed in a temporary on-site Satellite Accumulation Area (\#NTS087) until closure activities were completed. The total volume for these wastes was less than $0.5 \mathrm{~L}$ ( 0.13 gal) of liquids. Sampling of this small volume of waste to determine hazardous characteristics consumed the entire waste volume.

Waste disposition records, including the Uniform Hazardous Waste Manifest and NTS landfill documents, are available in NTS Waste Operations files and are summarized in Appendix B. 



\subsection{CLOSURE VERIFICATION RESULTS}

\subsection{VERIFICATION SAMPLE ANALYSES}

Verification soil samples were collected from 35 locations after the excavations reached designated boundaries and no visible staining was observed. The samples were collected with decontaminated stainless steel sampling scoops and placed in labeled sample containers secured with custody seals. The containers were placed in an ice-filled chest, transported under chain-ofcustody to the Analytical Services Laboratory in Mercury, Nevada, then forwarded to an off-site laboratory for analyses. Samples from all 35 locations were analyzed for total pesticides (EPA, 1996b). Samples from eight of these locations were also analyzed for total petroleum hydrocarbons and one was also analyzed for TCLP pesticides (EPA, 1996b).

Chlordane was the only pesticide EPA Industrial PRG exceeded (1,500 micrograms/kilogram [ug/kg]) in soil samples QH04 (2,200 ug/kg), SK05 (5,700 ug/kg), SK21 (5,800 ug/kg), and SK22 (5,000 ug/kg). After additional excavation, deeper soil samples were collected (QH17/QH18[duplicate], SK23, SK24, and SK25, respectively) which did not exceed pesticide PRGs. In the sample analyzed for TCLP pesticides (SK05), only Lindane was detected at a concentration $(0.3$ micrograms per liter $[\mathrm{ug} / \mathrm{L}])$ well below the TCLP level $(400 \mathrm{ug} / \mathrm{L})$. None of the eight samples analyzed for total petroleum hydrocarbons exceeded the NDEP Action Level of 100 milligrams per kilogram.

The analytical results verify that pesticides and petroleum hydrocarbons remaining in the ground are below EPA TCLP levels, EPA Industrial PRGs, and the NDEP Action Level. The analytical results for pesticides and petroleum hydrocarbons are summarized in Tables 2 and 3 , respectively, and the analytical reports are included in Appendix C.

\subsection{BACKFILLING AND REGRADING OF EXCAVATIONS}

Excavations were backfilled and/or regraded after verification sampling indicated clean closure was achieved. Clean backfill was placed and compacted by downward blows of the backhoe bucket (in areas of limited access) and/or by several passes of the loader or other heavy equipment. The site was then graded to restore natural drainage patterns and minimize ponding. A water truck was used to condition the borrow soil and for dust suppression. Verification testing of compaction was not required or performed. 


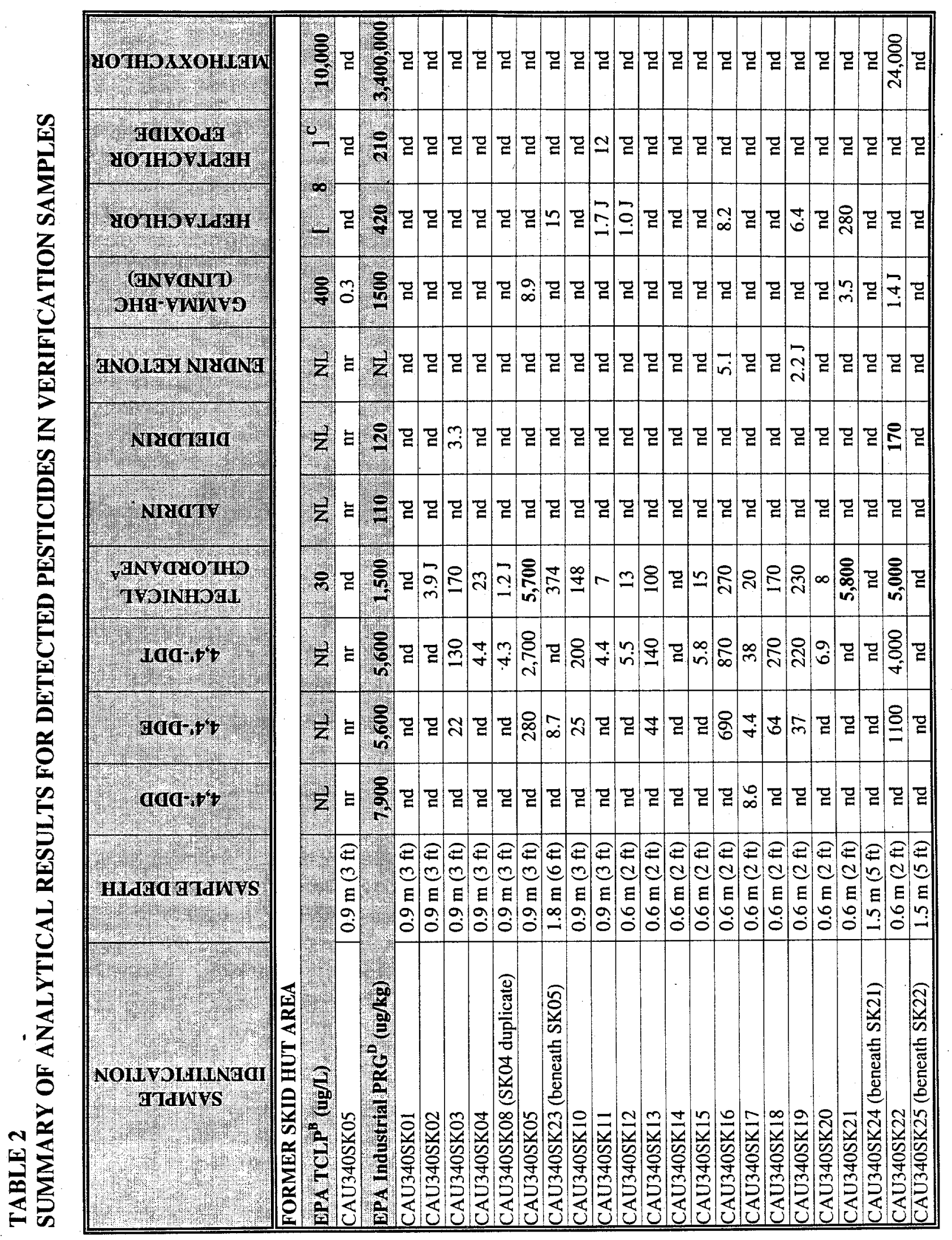


TABLE 2 (continued)

SUMMARY OF ANALYTICAL RESULTS FOR DETECTED PESTICIDES IN VERIFICATION SAMPLES

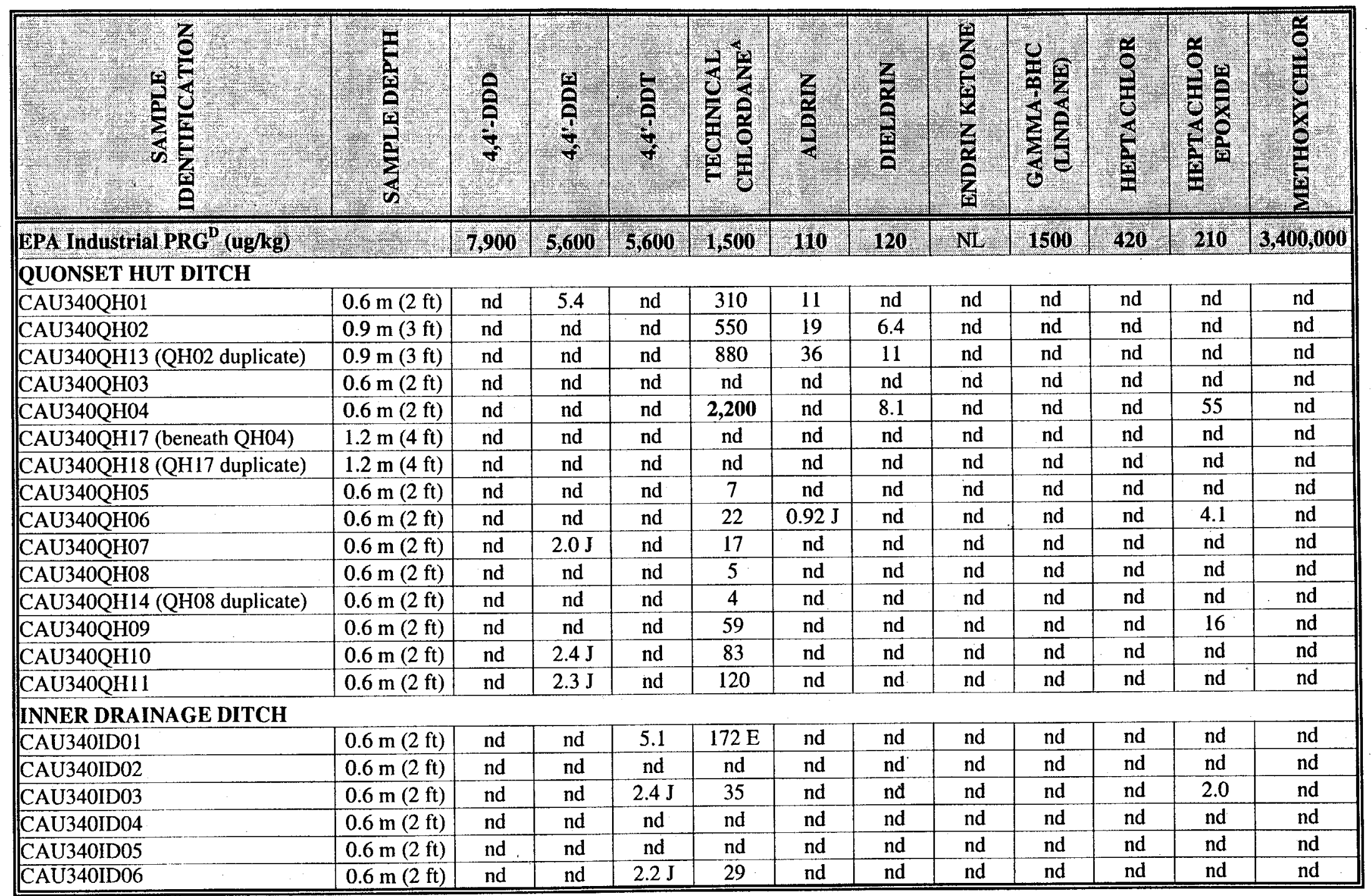


TABLE 2 (continued)

\section{SUMMARY OF ANALYTICAL RESULTS FOR DETECTED PESTICIDES IN VERIFICATION SAMPLES}

\section{Notes:}

Remediation-standard pesticides for CAU 340 were identified in the CADD (DOE, 1998) and CAP (DOE, 1999).

EPA Method SW846 8081 A (EPA, 1996b). See analytical reports in Appendix B for detection limits.

Analytical results for blank and $\mathrm{ms} / \mathrm{msd} \mathrm{QA} / \mathrm{QC}$ samples are not shown.

A - Technical Chlordane is the sum of chlordane, alpha-chlordane, and gamma-chlordane.

B - EPA Toxicity Characteristic Leachate Procedure (TCLP) for 1996 (EPA, 1996c).

C - Heptachlor and its epoxide.

D - EPA Region IX Industrial Preliminary Remediation Goals (PRGs) for 1996 (EPA, 1996a).

E - Value above quantitation range.

$\mathrm{ft}$ - Foot

$\mathrm{J}$ - Analyte detected below quantitation limit.

$\mathrm{m}$ - Meter

na - Not applicable.

nd - Not detected at the reporting limit.

NL - Not listed.

N $\quad n r-$ Not reported.

$\mathrm{ug} / \mathrm{kg}$ - Microgram per kilogram.

ug/L - Microgram per liter.

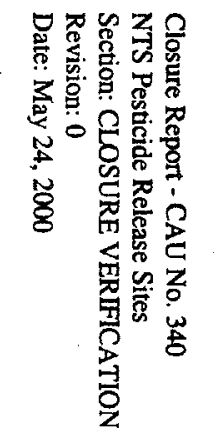


TABLE 3

SUMMARY OF ANALYTICAL RESULTS FOR TPH IN VERIFICATION SAMPLES

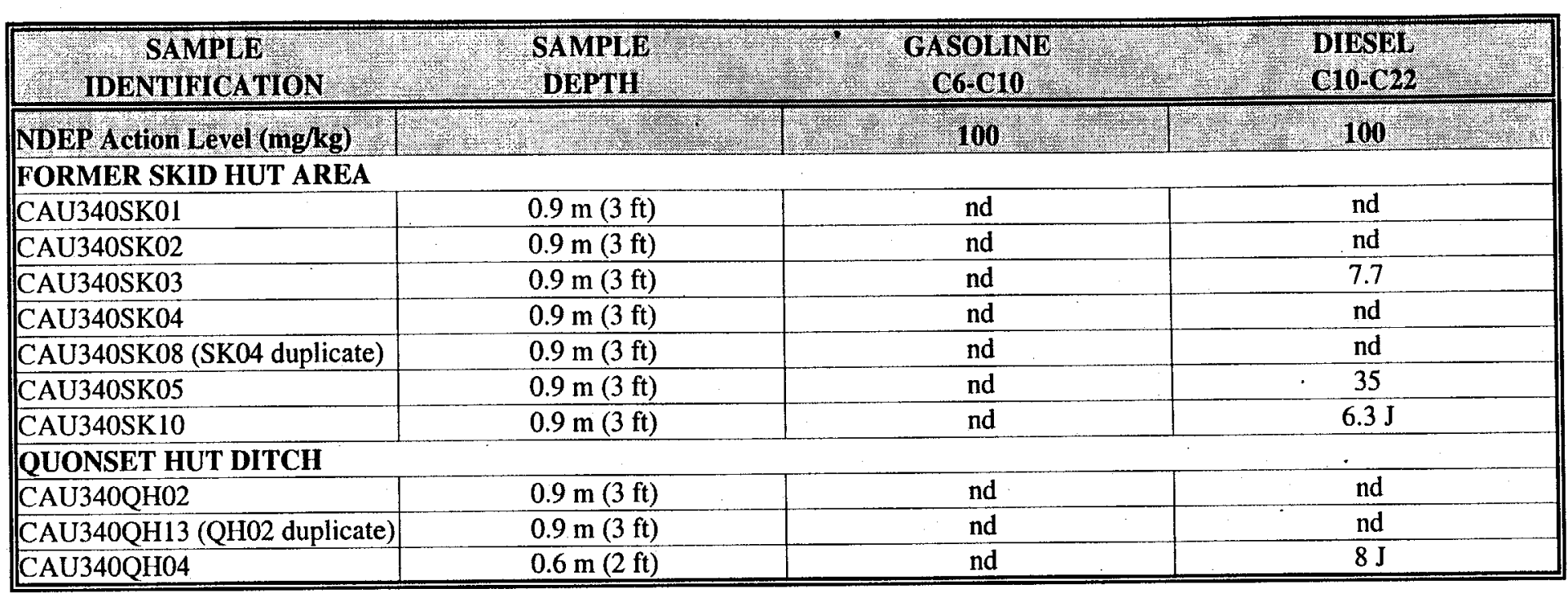

Notes:

EPA Method SW8015B (EPA, 1996b). See analytical reports in Appendix B for detection limits.

Analytical results for blank and $\mathrm{ms} / \mathrm{msd} \mathrm{QA} / \mathrm{QC}$ samples are not shown.

$\mathrm{ft}$ - Foot

$\mathrm{J}$ - Analyte detected below quantitation limit

m - Meter

$\mathrm{mg} / \mathrm{kg}$ - Milligram per kilogram

na - Not applicable

nd - Not detected at the reporting limit

TPH - Total petroleum hydrocarbons 
a 


\subsection{SUMMARY AND RECOMMENDATIONS}

\subsection{SUMMARY}

The following site closure activities were performed at the two CAU 340 sites located in Area 23 and are documented in this report:

- All soil containing pesticides at or above EPA TCLP hazardous-waste levels was removed and transported off-site to a TSDF.

- All soil containing pesticides at or above EPA Industrial PRGs (but below EPA TCLP hazardous-waste levels) was removed and disposed in the NTS Area 23 Mercury Landfill.

- All soil containing petroleum hydrocarbons at or above the NDEP Action Level was removed and disposed in the NTS Area 6 Hydrocarbon Landfill.

- The remaining skid huts and other debris were disposed in the NTS Area 9 U10c Landfill.

- The excavations were backfilled with borrow soil and/or regraded to promote drainage and minimize ponding of surface water.

- $\quad$ The field closure activities conducted at CAU 340 were completed following the approved CAP (DOE, 1999).

\subsection{RECOMMENDATIONS}

Since the closure activities for CAU 340 have been completed following the NDEP-approved CAP (DOE, 1999) as documented in this CR, the DOE requests:

- $\quad$ A Notice of Completion be provided by the NDEP to DOE for the closure of CAU 340 (CAS 15-18-02; CAS 23-21-01; and CAS 23-28-03).

- CAU 340 be moved from Appendix III to Appendix IV of the FFACO "Closed Corrective Action Units." 



\subsection{REFERENCES}

BN, see Bechtel Nevada.

Bechtel Nevada, 1999a. Field Management Plan for Corrective Action Unit 340: Area 23

Pesticide Release Site, Nevada Test Site, Nevada, Las Vegas, NV.

Bechtel Nevada, 1999b. Site Specific Health and Safety Plan for Corrective Action Unit 340: Area 23 Pesticide Release Site, Nevada Test Site, Nevada, Las Vegas, NV.

DOE, see U.S. Department of Energy.

EPA, see U.S. Environmental Protection Agency

FFACO, see Federal Facility Agreement and Consent Order.

Federal Facility Agreement and Consent Order of 1996. Prepared by Nevada Division of Environmental Protection, U.S. Department of Energy, and U.S. Department of Defense.

Nevada Administrative Code, 1996. NAC 445A.2272, "Contamination of soil: Establishment of action levels," As adopted by the Nevada Environmental Commission, October, Carson City, NV.

U.S. Department of Energy, Nevada Operations Office, 1998. Corrective Action Decision Document for Corrective Action Unit 340: Pesticide Release Sites, Nevada Test Site, Nevada, DOE/NV--525, Las Vegas, NV.

U.S. Department of Energy, Nevada Operations Office, 1999. Corrective Action Plan for Corrective Action Unit 340: Pesticide Release Site. Nevada Test Site, Nevada, DOE/NV--560, Las Vegas, NV.

U.S. Department of Energy, Nevada Operations Office, 2000. Nevada Test Site Resource Management Plan Annual Summary, January 2000, DOE/NV--604, Las Vegas; NV.

U.S. Environmental Protection Agency, 1996a, Region IX Preliminary Remediation Goals (PRGs), San Francisco, CA.

U.S. Environmental Protection Agency, 1996b, Test Methods for Evaluating Solid Waste, Physical/Chemical Methods, EPA Publication SW-846, Third Edition.

U.S. Environmental Protection Agency, 1996c, Title 40 Code of Federal Regulations 261.24, "Toxicity Characteristic," Washington, D.C. 




\section{APPENDIX A}

\section{PHOTOGRAPHS OF CLOSURE ACTIVITY WORK AREAS}





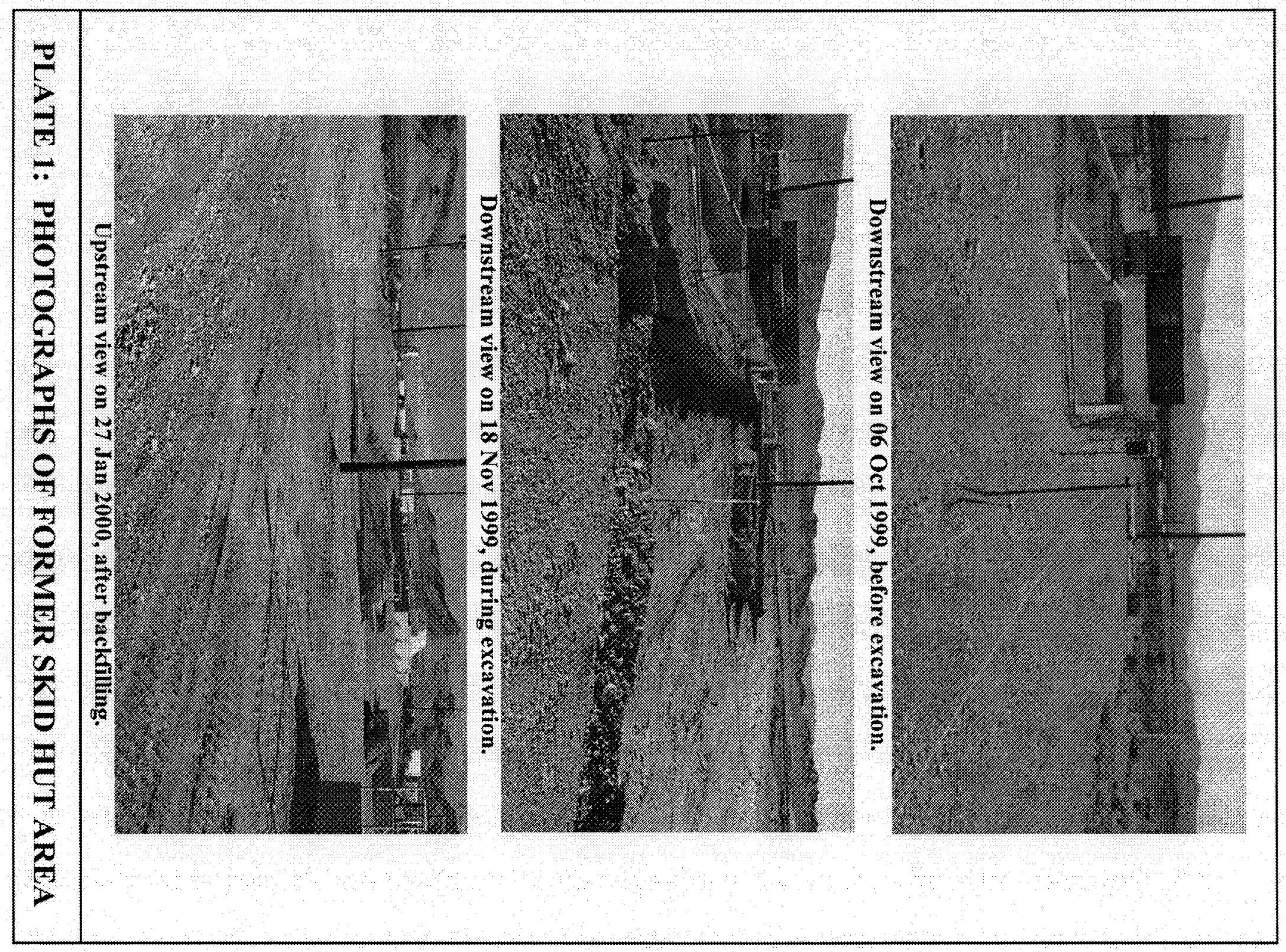




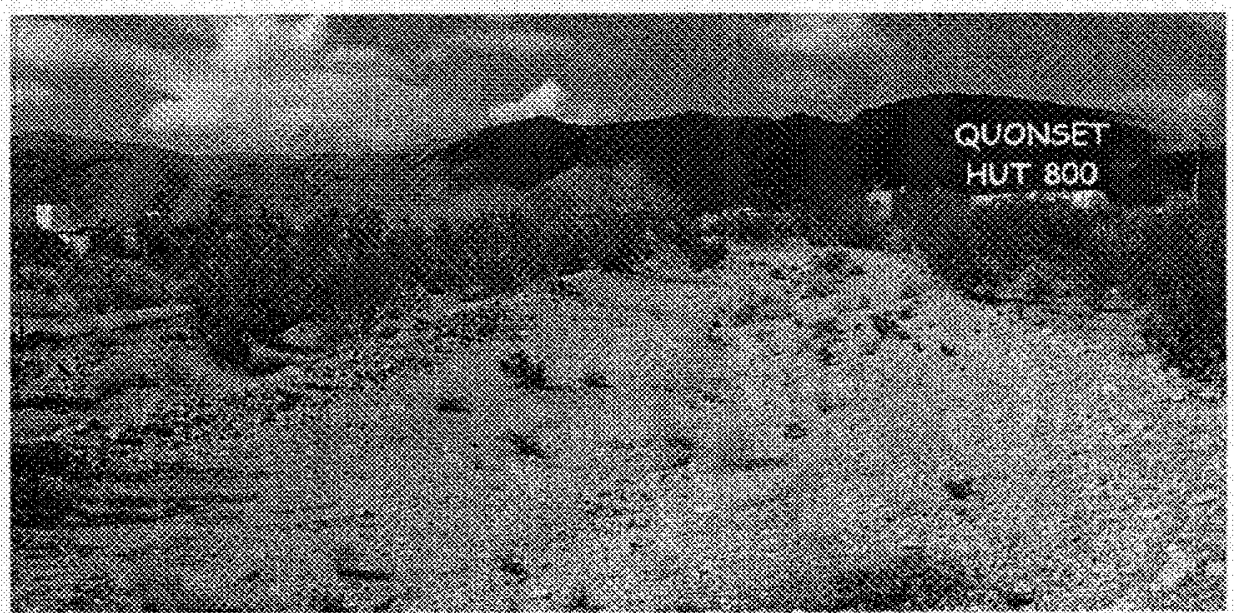

Upstream view on 06 Oct 1999, before excavation.

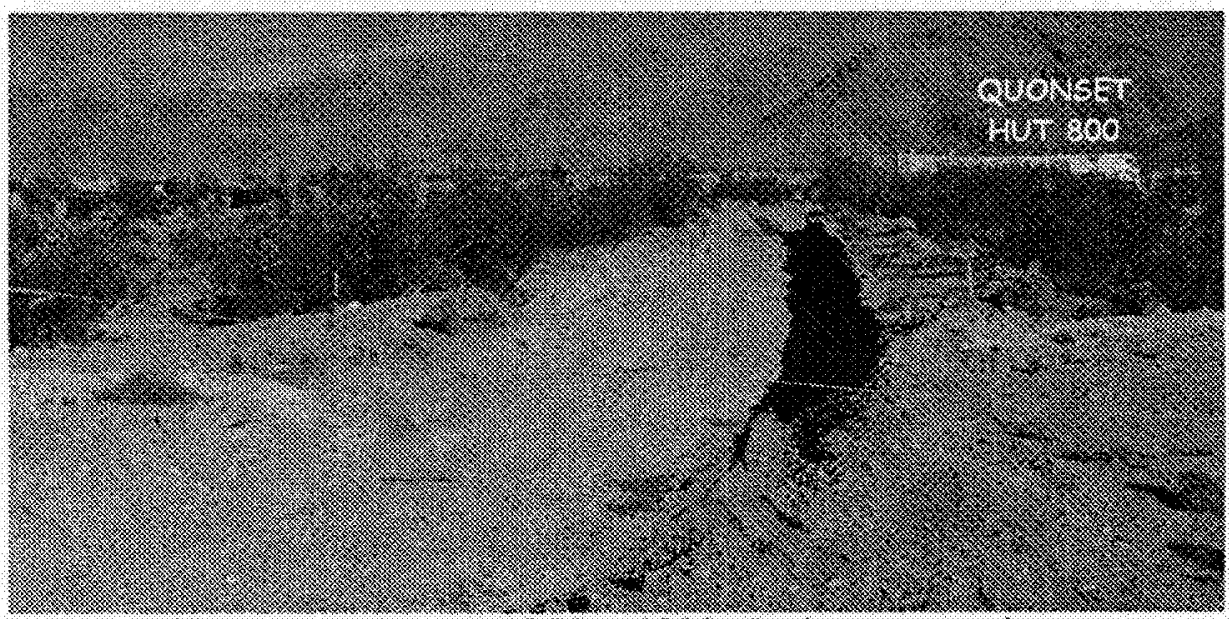

Upstream view on 18 Nov 1999 , during excavation.

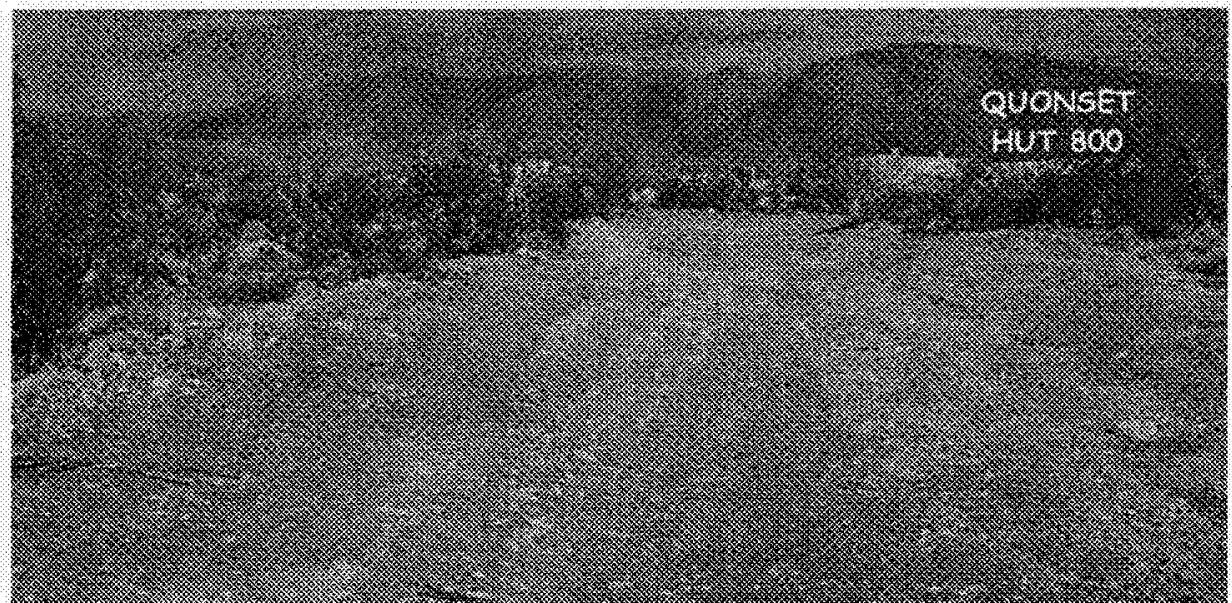

Upstream view on 27 Jan 2000 , after regrading.

PLATE 2: PHOTOGRAPHS OF QUONSET HUT DITCH 


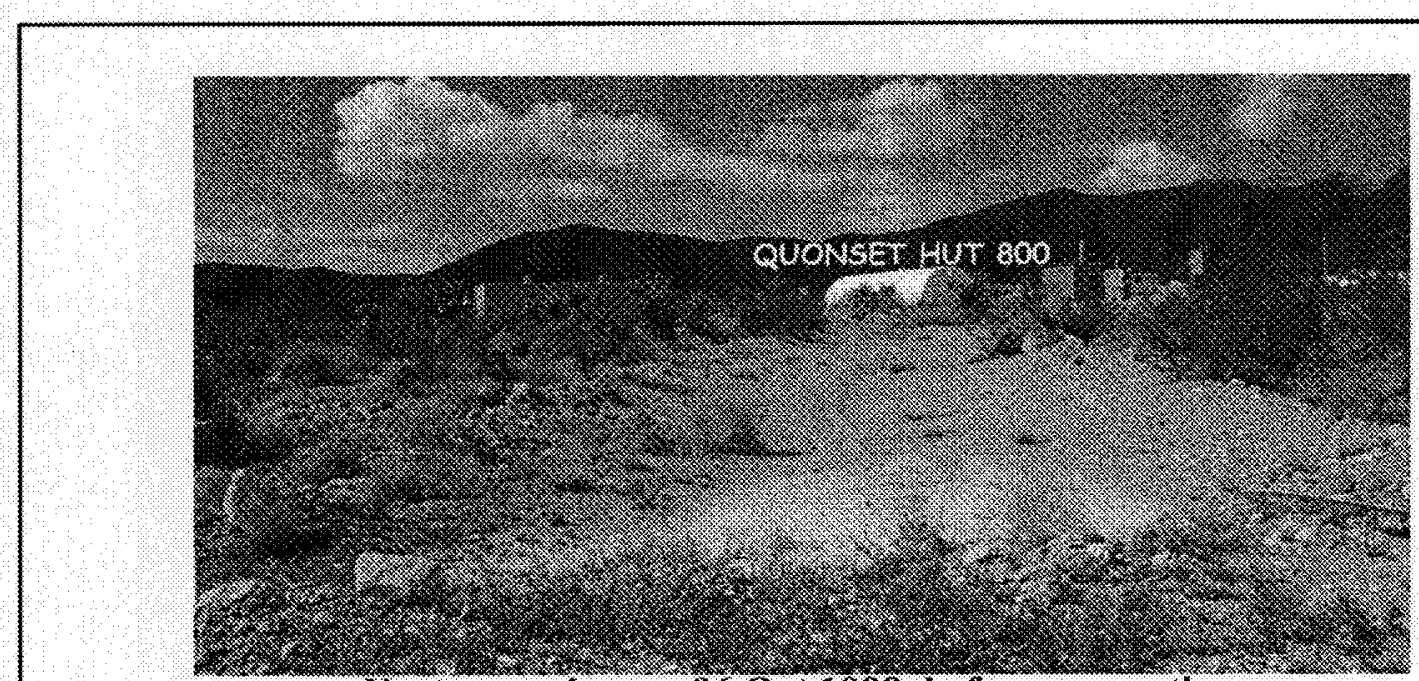

Upstream view on 06 Oet 1999, before excavation.

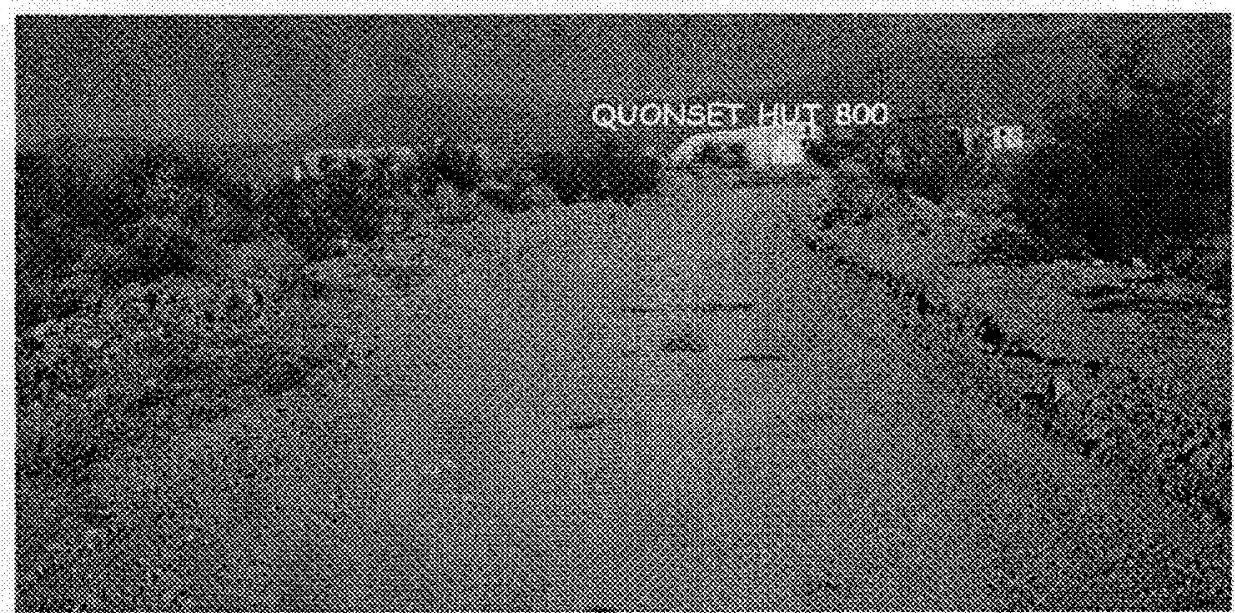

Upstream view on 18 Nov 1999, after excavation.

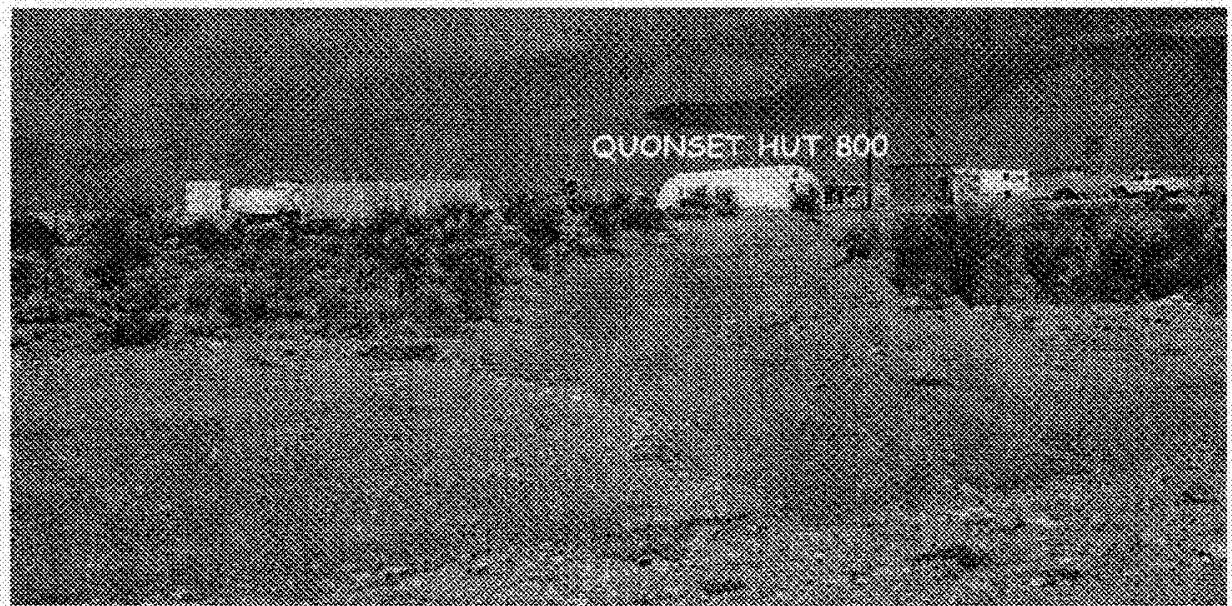

Upstream view on 27 Jan 2000 , after regrading.

PLATE 3: PHOTOGRAPHS OF INNER DRAINAGE DITCH 

APPENDIX B

\section{WASTE DISPOSITION DOCUMENTATION}



Interoffice Memorandum

To: W. F. Johnson

From: ${ }^{\circ} \quad$ C. A. Stowell, Project Manager Solid Waste Operations
Date: February 14, 2000

No.: 2150-CS-00-0025

Subject: Waste Disposal Documentation for Corrective Action Unit (CAU) 340 and 342 Project No. N/A

Between November 8, 1999 and January 13, 2000, Nevada Test Site (NTS) landfills received approximately 3,029 tons of waste from CAU 340 remediation activities.

- Approximately 71 tons of hydrocarbon impacted waste were disposed at the NTS Area 6 Hydrocarbon Class III Industrial Solid Waste Disposal Site (SWDS).

- Approximately 2,937 tons of waste were disposed at the NTS Area 23 Class II Municipal and Industrial SWDS.

- Approximately 21 tons of waste were disposed at the NTS Area 9 U10c Class II Industrial SWDS.

Additionally, on December 16, 1999, approximately 16 tons of hazardous waste from CAU 340 remediation activities was transported from the NTS to Aragonite, UT for disposal. Enclosed is a copy of the Uniform Hazardous Waste Manifest associated with this waste.

Between October 25, 1999 and November 3, 1999, the Area 6 Hydrocarbon Class III Industrial Solid Waste Disposal Site received approximately 2,118 tons of hydrocarbon impacted waste from CAU 342 remediation activities.

C. A. Stowell

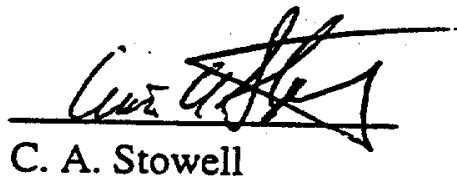

FEC/CAS:cjs

Subject Code: WMT3

Enclosures: as stated

cc w/o encs.

Correspondence Control, NLV008

J. M. Holden, NTS207

C. J. Schwartze, NTS207

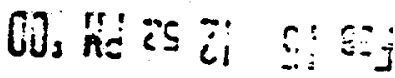




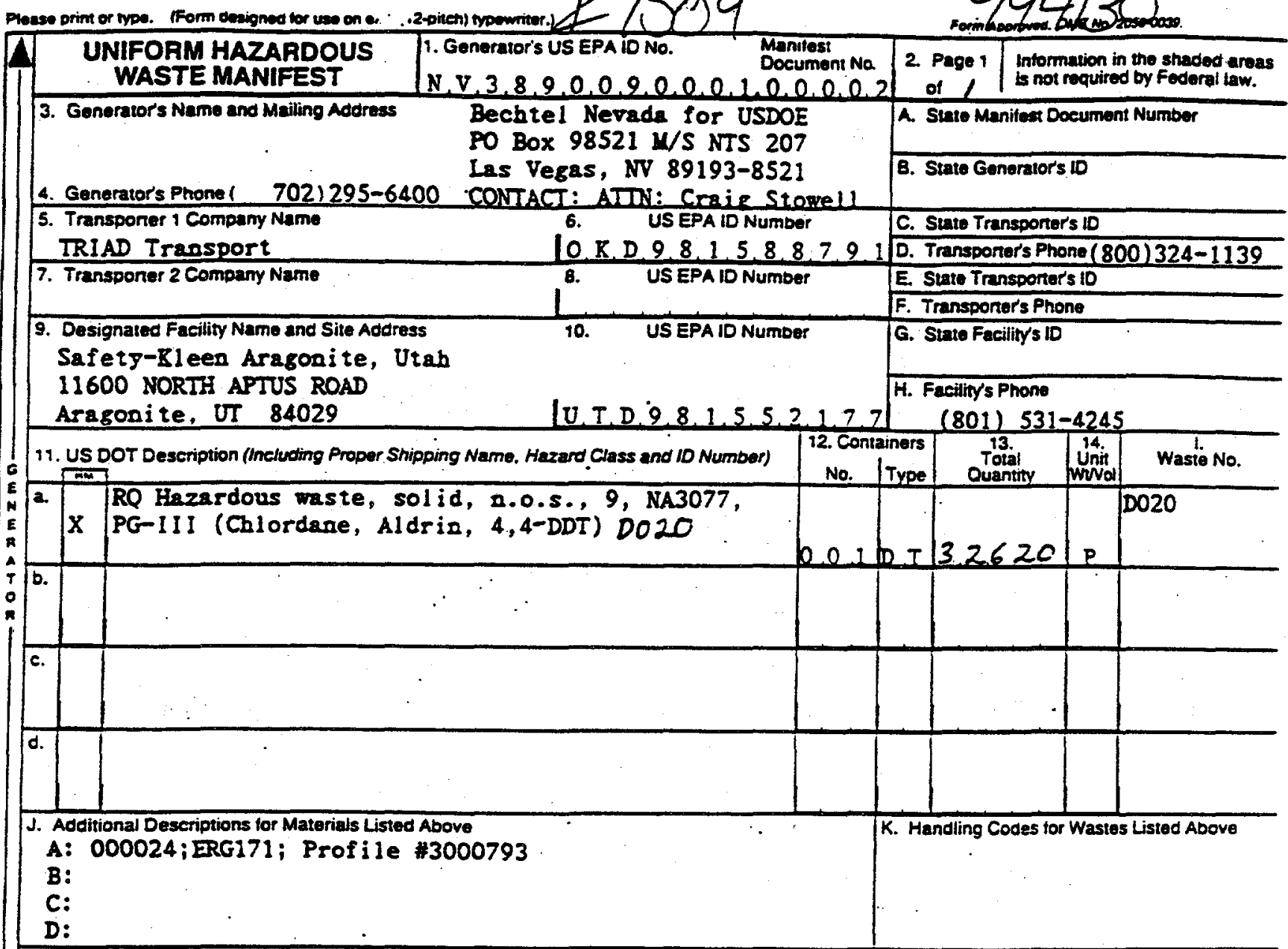

15. Special Haneling Instructions and Additional Information

24 HR EMERGENCY CONTACT \# (702) 295-6400

Certificate of Destruction is REQUIRED.

Use proper PPE when handling containers.

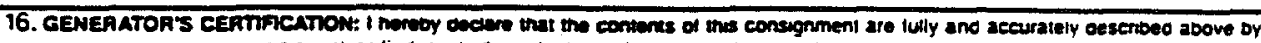

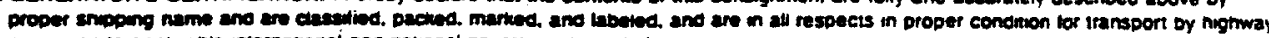

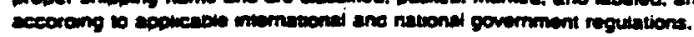

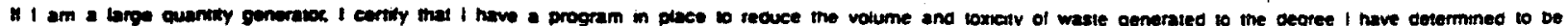

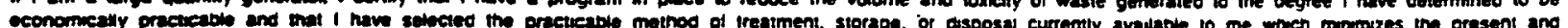

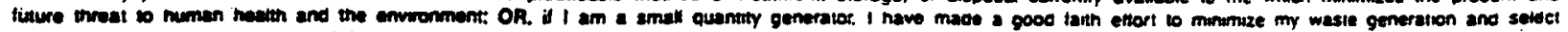

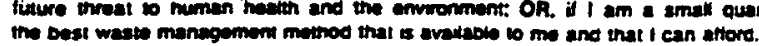

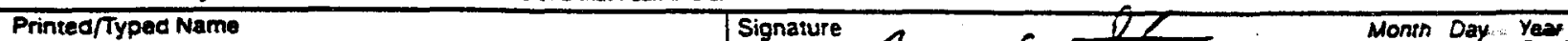

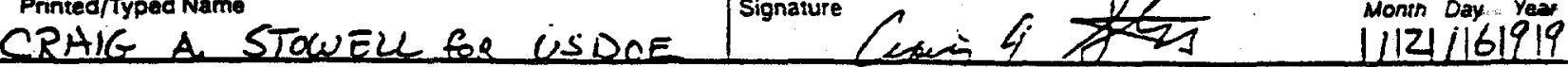

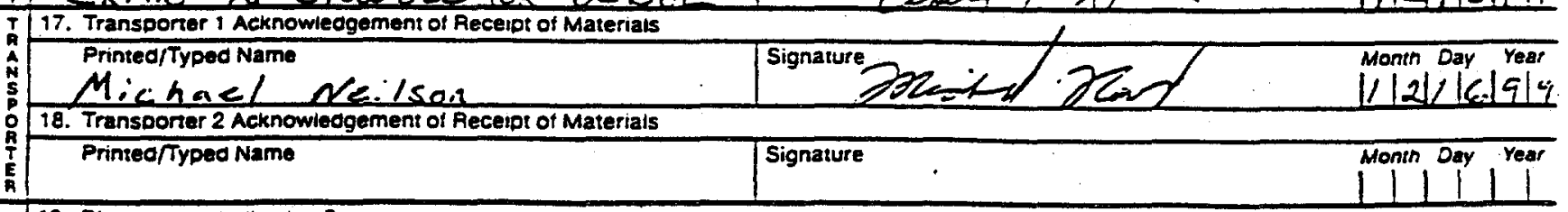

19. Discrepancy indieation Space

e.

20. Factity Owner or Operator: Certificatton of receidt of hazarcous materiais covered by this manifest except as noted in trem 19.

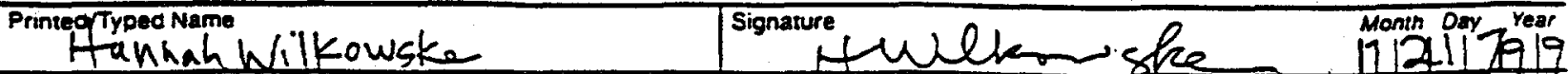

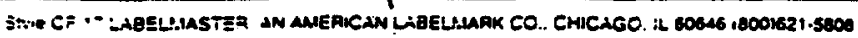





\section{APPENDIX C}

\section{VERIFICATION SAMPLE ANALYTICAL REPORTS}



Date: $07-$ Dec-99

Barringer Laboratories, Inc.

$15000 \mathrm{~W}$ 6 th Avenue Suite $300 \quad$ Golden, Colorado $80401.5047 \quad$ (800)654-0506 (303) 277-1687 Fax (303)277.1689

Ted Redding

Bechtel Nevada Corp.

P.O. Box 98521

MS NTS 273

Las Vegas, NV. 89193-8521.

Phone: 1-702-295-7220

Fax: 1-702-295-4773

Work Order: 9911097

- Project: SEIR CAU-340-1-0

Dear Ted Redding,

Barringer Laboratories received 7 samples on $11 / 11 / 99$ for the analyses presented in the following report.

There were no problems with the analyses and all data for associated QC met EPA or laboratory specifications except where noted in the Case Narrative.

If you have any questions regarding these test results, please feel free to call.
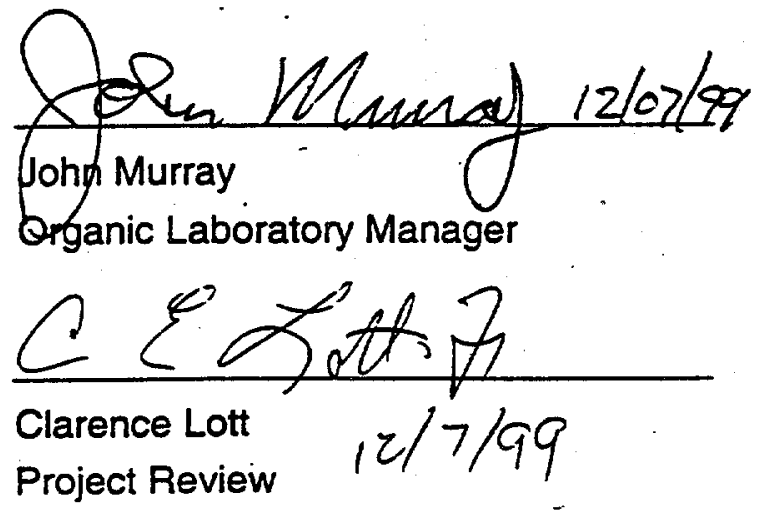

Date: $07-$ Dec-99

Barringer Laboratories, Inc.

$15000 \mathrm{~W}$ 6th Avenue Suike 300 Golden, Colorado 80401-5047

(800)654.0S06 (303) 277.1687 Fax (303) 277-1689

Client:

Bechtel Nevada Corp.

Project:

SEIR CAU-340-1-0

Work Order:

9911097

SAMPLE SUMMARY

Date Received: $11 / 11 / 99$

Temp Received: $4^{\circ} \mathrm{C}$

\begin{tabular}{|c|c|c|c|c|}
\hline Lab Sample ID & Client Sample ID & Tag Number Collection Date & Matrix & Bottle and Preservation \\
\hline $9911097-01 \mathrm{~A}$ & CAU340SKOI & $11 / 9 / 99$ & Soil & $80 z$ amber glass, unpreserved \\
\hline $9911097-02 A$ & CAU340SKO2 & $11 / 9 / 99$ & Soil & Boz amber glass, unpreserved \\
\hline $9911097-03 A$ & CAU340SKO3 & $11 / 9 / 99$ & Soil & $80 z$ amber glass, unpreserved \\
\hline $9911097.04 \mathrm{~A}$ & CAU340SKO4 & $11 / 9 / 99$ & Soil & $80 z$ amber glass, unpreserved \\
\hline $9911097-05 A$ & CAU340SKO5 & $11 / 9 / 99$ & Soil & $80 z$ amber giass, unpreserved \\
\hline $9911097-06 \mathrm{~A}$ & CAU340SKO8 & & Soil & $80 z$ amber glass, unpreserved \\
\hline $9911097-07 \mathrm{~A}$ & CAU340SKOS & $11 / 9 / 99$ & Aqueous & $1 \mathrm{~L}$ cubitainer, unpresenved \\
\hline $9911097-07 \mathrm{~B}$ & CAU3405KO9 & $11 / 9 / 99$ & Aqueous & $40 \mathrm{~mL}$ VOA vial, unpresened \\
\hline
\end{tabular}


Date: 07-Dec-99

Barringer Laboratories, Inc.

15000 W 6th Avenue Suire 300 Golden, Colorado 80401-5047 (800) 654-0506 (303) 277-1687 Fax (303) 277-1689

\begin{tabular}{lll}
\hline Client: & Bechtel Nevada Corp. & \\
Project: & SEIR CAU-340-1-0 & CASE NARRATIVE \\
Work Order: & 9911097 &
\end{tabular}

All reported values in this report have been rounded to the correct number of significant figures. All calculations have been performed before applying significant figures, therefore, not all calculations may be reproducible with the results printed in this report.

Analytical Comments for method SW846 8081A: There are some analytes that are being reported as estimated due to the analyte being above our calibration curve. The client is aware of this and does not require the samples analyzed at the required dilution to bring all analytes within the calibration range.

The temperature range for the TCLP extraction was 20-22C.

Analytical Comments for method SW8015BDRO, sample 9911097-05A: There was an interfering peak that coeluted with the surrogate which caused the recovery to exceed the set $Q C$ limit.

Analytical Comments for METHOD SW846 8015BGRO, SAMPLE 9911097-07BMSMSD: The normal concentration of the matrix spike is normally $1.5 \mathrm{ug} / \mathrm{mL}$ but were spiked at $2.0 \mathrm{ug} / \mathrm{ml}$ by accident. There was insufficeint sample for respiking. Therefore the recoveries reported for this matrix spike, matrix spike duplicate reflect the actual spiking concentration of $2.0 \mathrm{ug} / \mathrm{mL}$.

Analytical Comments for METHOD SW846 8015BGRO, SAMPLE MBLK: The surrogate exceeded the set QC limit for the method blank which was non-detect for gasoline. Because the surrogate was high we feel we could have detected gasoline if present and therefore consider the data to be valid as reported.

This report has been amended from the report dated 11/30/1999 to include the cilutions of the samples that were previously reported with estimated results for method SW846-8081A.

Analytical Comments for METHOD SW846 8081A, SAMPLE 9911097-03A: The result for gamma-Chlordane was taken from the second column because of an interfering peak on the primary column.

Analytical Comments for METHOD SW846 8081A, SAMPLE 9911097-05A: The result for gamma-Chlordane was taken from the second column because of an interfering peak on the primary column. 
Date: $07-$ Dec-99 Barringer Laboratories, Inc.

CLIENT SAMPLE REPORT

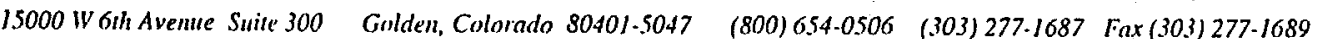

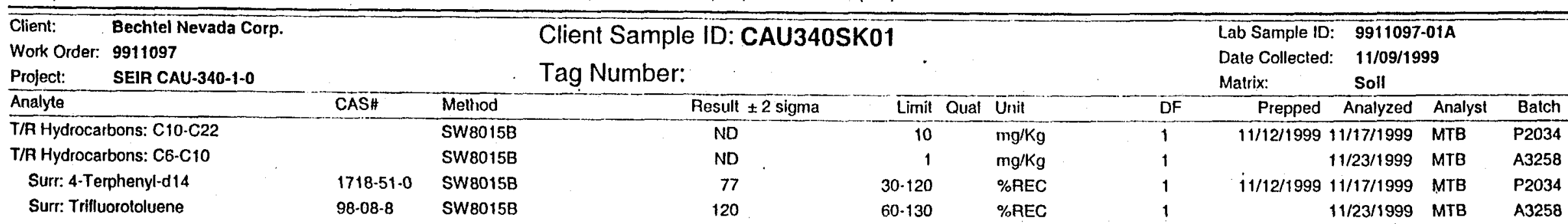

\begin{tabular}{|c|c|c|}
\hline $4,4^{\circ}-\mathrm{DDD}$ & $72 \cdot 54-8$ & SW846 8081A \\
\hline $4,4^{\circ}$-DDE & $72-55-9$ & SW846 8081A \\
\hline $4,4^{\circ} \cdot$ DDT & $50-29-3$ & SW846 8081A \\
\hline Aldrin & $309-00-2$ & SW846 8081A \\
\hline alpha-BHC & $319-84.6$ & SW846 8081A \\
\hline alpha-Chlordane & 5103.71 .9 & SW846 8081A \\
\hline beta-BHC & $319 \cdot 85 \cdot 7$ & SWB46 $8081 A$ \\
\hline Chlordane & $57-74-9$ & SW846 8081A \\
\hline della-BHC & $319-86-8$ & SW846 8081A \\
\hline Dieldrin & $60-57-1$ & SW846 8081A \\
\hline Endosulfan I & $959-98-8$ & SW846 B081A \\
\hline Endosulfan II & $33212-65-9$ & SW846 8081A \\
\hline Endosulfan sulfate & $1031-07-8$ & SW846 B081A \\
\hline Endrin & $72 \cdot 20 \cdot 8$ & SW846 $8081 A$ \\
\hline Endrin aidehyde & $7421-93-4$ & SW846 8081A \\
\hline Endrin ketone & $53494-70-5$ & SW846 8081A \\
\hline gamma-BHC & $58-89-9$ & SW846 8081A \\
\hline gamma.Chlordane & $5566-34 \cdot 7$ & SW846 8081A \\
\hline Heptachlor & $76-44-8$ & SW846 8081A \\
\hline Heplachlor epoxide & $1024-57-3$ & SW846 $8081 A$ \\
\hline Methoxychlor & $72-43-5$ & SW846 8081A \\
\hline Toxaphene & $8001-35-2$ & SW846 8081A \\
\hline Surr: Decachlorobiphenyl & 2051-24-3 & SW846 8081A \\
\hline Surr: Tetrachloro-m-xylene & $877-09-8$ & SWB46 8081A \\
\hline
\end{tabular}

\begin{tabular}{|c|c|c|}
\hline ND & 3 & $\mu g / \mathrm{Kg}$ \\
\hline ND & 3 & $\mu g / K g$ \\
\hline ND & 3 & $\mu g / \mathrm{Kg}$ \\
\hline ND & 2 & $\mu g / K g$ \\
\hline ND & 2. & $\mu g / \mathrm{Kg}$ \\
\hline ND & 2 & $\mu g / \mathrm{Kg}$ \\
\hline ND & 2 & $\mu g / \mathrm{Kg}$ \\
\hline ND & 10 & $\mu g / \mathrm{Kg}$ \\
\hline ND & 2 & $\mu g / \mathrm{Kg}$ \\
\hline NO & 3 & $\mu g / \mathrm{Kg}$ \\
\hline ND & 2 & $\mu g / \mathrm{Kg}$ \\
\hline ND & 3 & $\mu \mathrm{g} / \mathrm{Kg}$ \\
\hline ND & 3 & $\mu g / \mathrm{Kg}$ \\
\hline ND & 3 & $\mu \mathrm{g} / \mathrm{Kg}$ \\
\hline ND & 3 & $\mu g / \mathrm{Kg}$ \\
\hline ND & 3 & $\mu \mathrm{g} / \mathrm{Kg}$ \\
\hline ND & 2 & $\mu g / \mathrm{Kg}$ \\
\hline ND & 2 & $\mu g / \mathrm{Kg}$ \\
\hline ND & 2 & $\mu g / K g$ \\
\hline ND & 2 & $\mu \mathrm{g} / \mathrm{kg}$ \\
\hline ND & 20 & $\mu g / \mathrm{Kg}$ \\
\hline ND & 200 & $\mu g / \mathrm{Kg}$ \\
\hline 106 & $50-163$ & \%REC \\
\hline 87 & $31-115$ & $\%$ REC \\
\hline
\end{tabular}

\begin{tabular}{|c|c|c|}
\hline $11 / 11 / 1999$ 11/13/1999 & $\mathrm{RDH}$ & P1993 \\
\hline $11 / 11 / 1999 \quad 11 / 13 / 1999$ & $\mathrm{ADH}$ & P1993 \\
\hline $11 / 11 / 1999 \quad 11 / 13 / 1999$ & RDH & P1993 \\
\hline $11 / 11 / 199911 / 13 / 1999$ & $\mathrm{ROH}$ & P1993 \\
\hline $11 / 11 / 1999$ 11/13/1999 & RDH & P1993 \\
\hline $11 / 11 / 199911 / 13 / 1999$ & RDH & P1993 \\
\hline $11 / 11 / 1999$ 11/13/1999 & RDH & P1993 \\
\hline $11 / 11 / 1999$ 11/13/1999 & RDH & P1993 \\
\hline $11 / 11 / 199911 / 13 / 1999$ & RDH & P1993 \\
\hline 11/11/1999 11/13/1999 & $\mathrm{RDH}$ & P1993 \\
\hline 11/11/1999 11/13/1999 & RDH & P1993 \\
\hline $11 / 11 / 199911 / 13 / 1999$ & RDH & P1993 \\
\hline 11/11/1999 11/13/1999 & RDH & P1993 \\
\hline $11 / 11 / 1999+11 / 13 / 1999$ & $\mathrm{RDH}$ & P1993 \\
\hline $11 / 11 / 1999$ 11/13/1999 & $\mathrm{ROH}$ & P1993 \\
\hline $11 / 11 / 1999$ 11/13/1999 & RDH & P1993 \\
\hline 11/11/1999 11/13/1999 & $\mathrm{RDH}$ & P1993 \\
\hline 11/11/1999 11/13/1999 & $\mathrm{RDH}$ & P1993 \\
\hline $11 / 11 / 1999$ 11/13/1999 & RDH & P1993 \\
\hline $11 / 11 / 199911 / 13 / 1999$ & $\mathrm{ROH}$ & P1993 \\
\hline 11/11/1999 11/13/1999 & ADH & P1993 \\
\hline 11/11/1999 11/13/1999 & $\mathrm{ROH}$ & P1993 \\
\hline 11/11/1999 11/13/1999 & RDH & 1993 \\
\hline $11 / 13 / 15$ & ADH & P1993 \\
\hline
\end{tabular}

$\begin{array}{ll}\text { Qualilicrs: } & \text { ND - Not delected at the reporting limit } \\ & \mathrm{J} \text { - Analyte detecled below quantitation limits } \\ & \mathrm{E}-\text { Value above quantitation range }\end{array}$
$R \cdot$ - RPD outside accepted recovery limils

$X$ - Duplicate sample(s) $<5$ times limil

$S$ - Spike recovery outside accepted recovery limits
$Y$ - Unspiked sample $>4$ times amount spiked

B - Analyte detected in the associaled method blank

$Z$ - Sample $>10$ times blank result 
Date: $07-$ Dec-99 Barringer Laboratories, Inc.

CLIENT SAMPLE REPORT

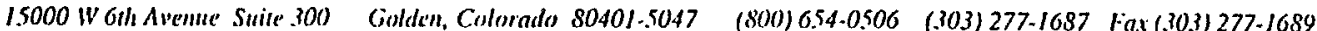

\begin{tabular}{|c|c|c|c|c|c|c|c|c|c|c|c|c|}
\hline $\begin{array}{l}\text { Client: } \\
\text { Work Order: } \\
\text { Project: }\end{array}$ & $\begin{array}{l}\text { Bechtel Nevada Corp. } \\
9911097 \\
\text { SEIR CAU-340-1-0 }\end{array}$ & \multicolumn{6}{|c|}{$\begin{array}{l}\text { Client Sample ID: CAU340SK02 } \\
\text { Tag Number: }\end{array}$} & \multicolumn{5}{|c|}{$\begin{array}{ll}\text { Lab Sample ID: } & 9911097-02 A \\
\text { Date Collected: } & 11 / 09 / 1999 \\
\text { Matrix: } & \text { Soll } \\
\end{array}$} \\
\hline Analyte & & CASH & Method & Result \pm 2 sigma & Limit & Qual & Unit & DF & Prepped & Analyzed & Analyst & Batch \\
\hline T/R Hydrocar & bons: $\mathrm{C} 10-\mathrm{C} 22$ & . & SW8015B & ND & 10 & & $\mathrm{mg} / \mathrm{Kg}$ & 1 & $11 / 12 / 1999$ & $11 / 17 / 1999$ & MTB & P2034 \\
\hline T/R Hydrocar & bons: C6-C10 & & SW8015B & ND & 1 & & $\mathrm{mg} / \mathrm{Kg}$ & 1 & & 11/23/1999 & MTB & A325B \\
\hline Surr: 4-Ter & phenyl-d14 & $1718-51-0$ & SW8015B & 86 & $30-120$ & & $\%$ REC & 1 & $11 / 12 / 1999$ & $11 / 17 / 1999$ & MTB & P2034 \\
\hline Surr: Triflu & orotoluene & $98-08-8$ & SW8015B & $118^{\circ}$ & $60 \cdot 130$ & & $\%$ REC & 1 & & $11 / 23 / 1999$ & MTB & A3258 \\
\hline $4,4: D D D$ & & $72 \cdot 54-8$ & SW846 8081A & ND & 3 & & $11 \mathrm{~g} / \mathrm{Kg}$ & 1 & . 11/11/1999 & $11 / 13 / 1999$ & $\mathrm{RDH}$ & P1993 \\
\hline $4,4^{\circ} \cdot \mathrm{DDE}$ & & $72-55-9$ & SW846 8081A & ND & 3 & & $\mu \mathrm{g} / \mathrm{Kg}$ & 1 & $11 / 11 / 1999$ & $11 / 13 / 1999$ & $\mathrm{RDH}$ & P1993 \\
\hline $4,4^{\circ}-\mathrm{DDT}$ & & $50-29-3$ & SW8468081A & ND & 3 & & $\mu \mathrm{g} / \mathrm{Kg}$ & 1 & $11 / 11 / 1999$ & $11 / 13 / 1999$ & $\mathrm{RDH}$ & P1993 \\
\hline Aldrin & & $309-00-2$ & SW846 8081A & ND & 2 & & $\mu g / K g$ & 1 & $11 / 11 / 1999$ & $11 / 13 / 1999$ & $\mathrm{RDH}$ & P1993 \\
\hline alpha-BHC & & $319-84-6$ & SW846 8081A & ND & 2 & & $\mu \mathrm{g} / \mathrm{Kg}$ & 1 & $11 / 11 / 1999$ & $11 / 13 / 1999$ & $\mathrm{RDH}$ & P1993 \\
\hline alpha-Chlord & lane & $5103-71.9$ & SW846 $8081 \mathrm{~A}$ & 1.7 & 2 & 9 & $\mu g / K g$ & 1 & $11 / 11 / 1999$ & $11 / 13 / 1999$ & $\mathrm{ADH}$ & P1993 \\
\hline beta-BHC & & $319-85-7$ & SW846 8081A & ND & 2. & & $\mu g / \mathrm{kg}$ & 1 & $11 / 11 / 1999$ & $11 / 13 / 1999$ & $\mathrm{RDH}$ & P1993 \\
\hline Chlordane & & 57.74 .9 & SW846 8081A & ND & 10. & & $\mu \mathrm{g} / \mathrm{Kg}$ & 1 & $11 / 11 / 1999$ & $11 / 13 / 1999$ & $\mathrm{AOH}$ & P1993 \\
\hline delta-BHC & & $319-86-8$ & SW846 8081A & ND & 2 & & $\mu g / \mathrm{Kg}$ & 1 & $11 / 11 / 1999$ & $11 / 13 / 1999$ & $\mathrm{RDH}$ & P1993 \\
\hline Dieldrin & & $60-57-1$ & SW846 BOB1A & ND & 3 & & $\mu g / K g$ & 1 & $11 / 11 / 1999$ & $11 / 13 / 1999$ & $\mathrm{RDH}$ & P1993 \\
\hline Endosultan I & & $959-98-8$ & SW846 8081A & ND & 2 & & $\mathrm{Hg} / \mathrm{Kg}$ & 1 & $11 / 11 / 1999$ & $11 / 13 / 1999$ & RDH & P1993 \\
\hline Endosullan II & & $33212-65-9$ & SW846 8081A & ND & 3 & & $\mu g / K g$ & 1 & $11 / 11 / 1999$ & $11 / 13 / 1999$ & RDH & P1993 \\
\hline Endosulfan s & sulfate & $1031-07-8$ & SW846 8081A & ND & 3 & & $\mu g / \mathrm{Kg}$ & 1 & $11 / 11 / 1999$ & $11 / 13 / 1999$ & $\mathrm{RDH}$ & P1993 \\
\hline Endrin & & $72 \cdot 20-8$ & SW846 8081A & ND & 3 & & $\mu g / \mathrm{Kg}$ & 1 & $11 / 11 / 1999$ & $11 / 13 / 1999$ & $\mathrm{RDH}$ & P1993 \\
\hline Endrin aldehy & yde & $7421-93-4$ & SW846 8081A & ND & 3 & & $\mu \mathrm{g} / \mathrm{Kg}$ & 1 & $11 / 11 / 1999$ & $11 / 13 / 1999$ & RDH & P1993 \\
\hline Endrin keton & & $53494-70-5$ & SW846 8081A & ND & 3 & & $\mu g / \mathrm{Kg}$ & 1. & $11 / 11 / 1999$ & $11 / 13 / 1999$ & $\mathrm{RDH}$ & P1993 \\
\hline gamma-BHC & & $58-89-9$ & SW846 8081A & No & 2 & & $\mu g / \mathrm{Kg}$ & 1 & $11 / 11 / 1999$ & $11 / 13 / 1999$ & RDH & P1993 \\
\hline gamma-Chlo & rdane & $5566-34-7$ & SW846 8081A & 2.2 & 2 & & $\mu g / \mathrm{Kg}$ & 1 & $11 / 11 / 1999$ & $11 / 13 / 1999$ & $\mathrm{RDH}$ & :P4993 \\
\hline Heptachlor & & $76 \cdot 44-8$ & SW846 8081A & ND & 2 & & $\mu \mathrm{g} / \mathrm{Kg}$ & 1 & $11 / 11 / 1999$ & $11 / 13 / 1999$ & $\mathrm{RDH}$ & P1993 \\
\hline Heptachlor e & poxide & $1024-57-3$ & SW846 8081A & ND & 2 & & $\mu g / \mathrm{Kg}$ & 1 & $11 / 11 / 1999$ & $11 / 13 / 1999$ & $\mathrm{RDH}$ & P1993 \\
\hline Methoxychlor & & $72-43-5$ & SW846 $8081 A$ & ND & 20 & & $\mu g / \mathrm{Kg}$ & 1 & $11 / 11 / 1999$ & $11 / 13 / 1999$ & $\mathrm{RDH}$ & P1993 \\
\hline Toxaphene & & $8001-35-2$ & SW846 8081A & ND & 200 & & $\mu \mathrm{g} / \mathrm{Kg}$ & 1 & $11 / 11 / 1999$ & $11 / 13 / 1999$ & $\mathrm{RDH}$ & P1993 \\
\hline Surr: Deca & achlorobiphenyl & $2051-24 \cdot 3$ & SW846 8081A & 108 & $50-163$ & & $\%$ PEC & 1 & $11 / 11 / 1999$ & $11 / 13 / 1999$ & RDH & P1993 \\
\hline Surr: Tetre & achloro-m-xylene & $877-09-8$ & SW846 8081A & 91 & $31-115$ & & $\%$ REC & 1 & $11 / 11 / 1999$ & $11 / 13 / 1999$ & $\mathrm{RDH}$ & P1993 \\
\hline
\end{tabular}

Qualifiers: ND - Not detected at the reporting limit

$J$ - Analyte detecled below quantilation limits

E - Value above quantitation range
R - RPD outside accepted recovery limits

$X$ - Duplicate sample $(s)<5$ times limit

$S$ - Spike recovery outside accepted recovery limits
$Y$ - Unspiked sample $>4$ times amount spiked

$B$ - Analyte detected in the associated method blank

$Z$ - Sample $>10$ times blank result 
Date: $07-$ Dec-99 Barringer Laboratories, Inc.

CLIENT SAMPLE REPORT

15000 W Gth Avemue Suite 300 Golden, Colorado 80401-5047 (800) 6.54-0506 (30.3) 277-1687 Fax (30.3)277-1689

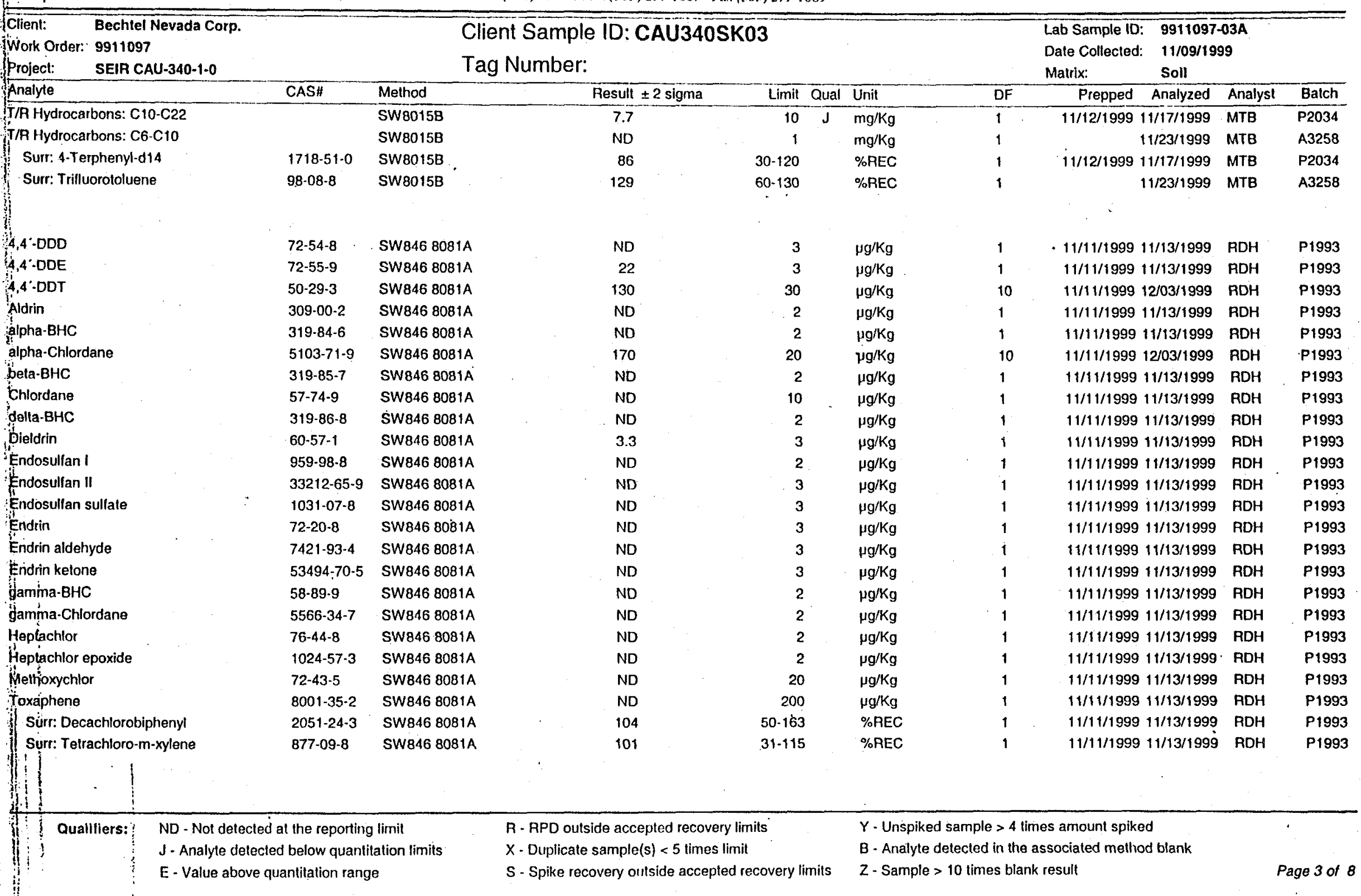


Date: 07-Dec-99

15000 W Gth Avenue Strite 300 Golden, Colorado 80401-5047 (800) 054-0.506 (303) 277-1687 Fax (303) 277.1689

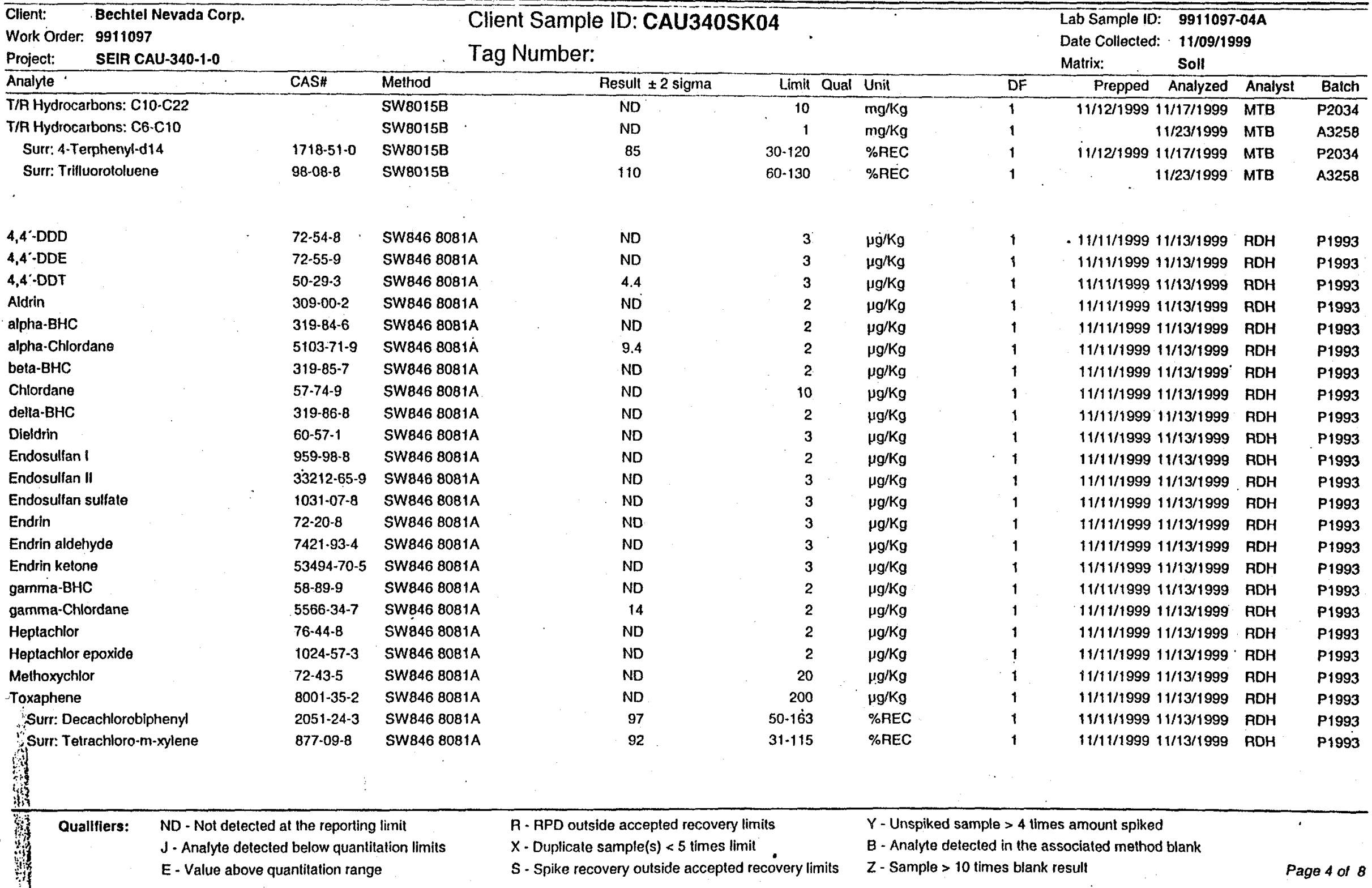


Date: 07-Dec-99 Barringer Laboratories, Inc.

CLIENT SAMPLE REPORT

15000 W' Gih Aveme Suite 300 Golden, Colorado 80401-5047 $\quad$ (800) 654-0506 (30.3) 277-1687 Fax (30.3) 277-1689

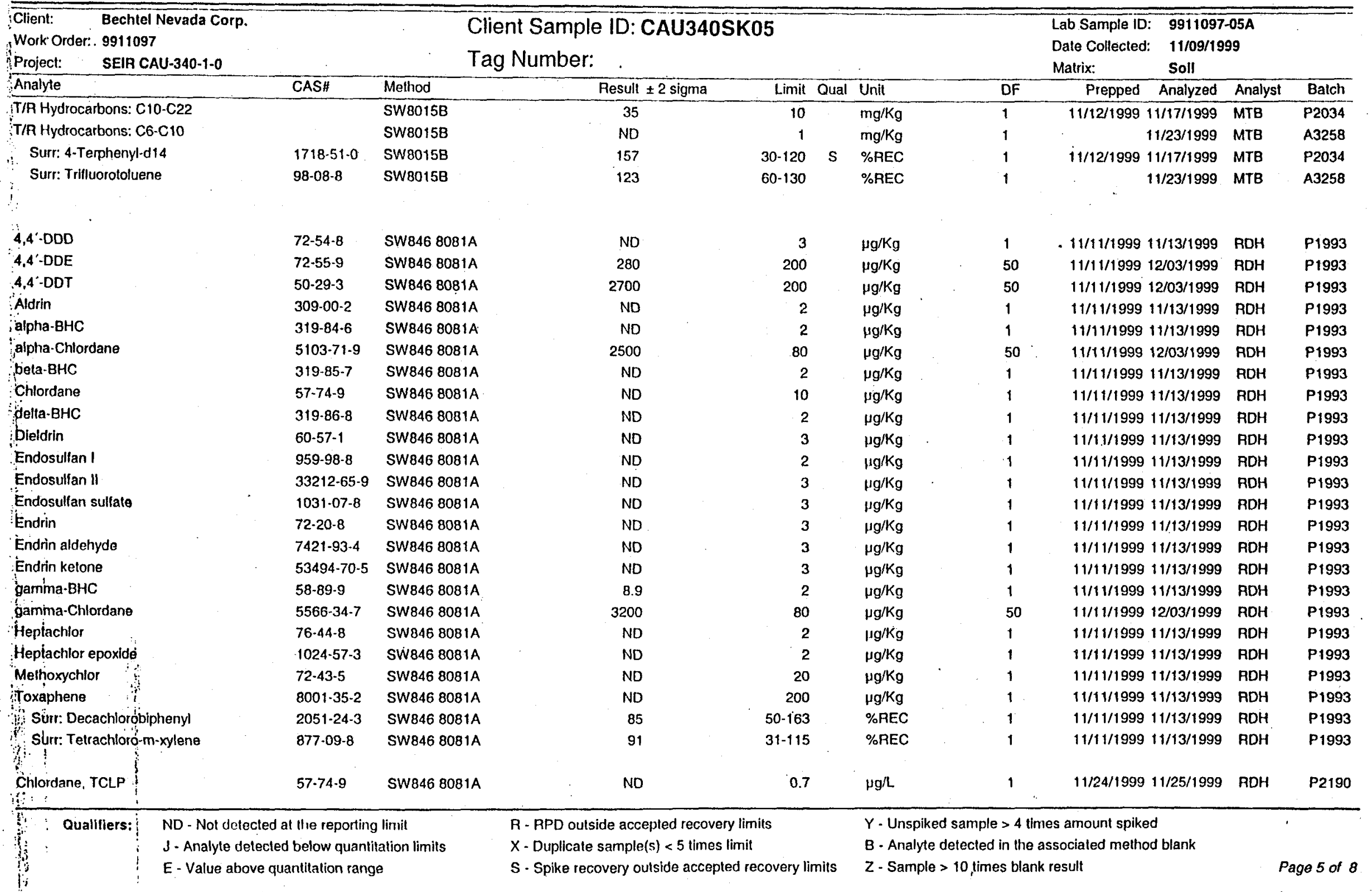


Date: 07-Dec-99 Barringer Laboratories, Inc.

CLIENT SAMPLE REPORT

'I5000 W Glh Avenue Suite 300 Golden, Colorado 80401-5047 (800)654-0506 (303) 277-1687 Fax (30.3) 277-1689

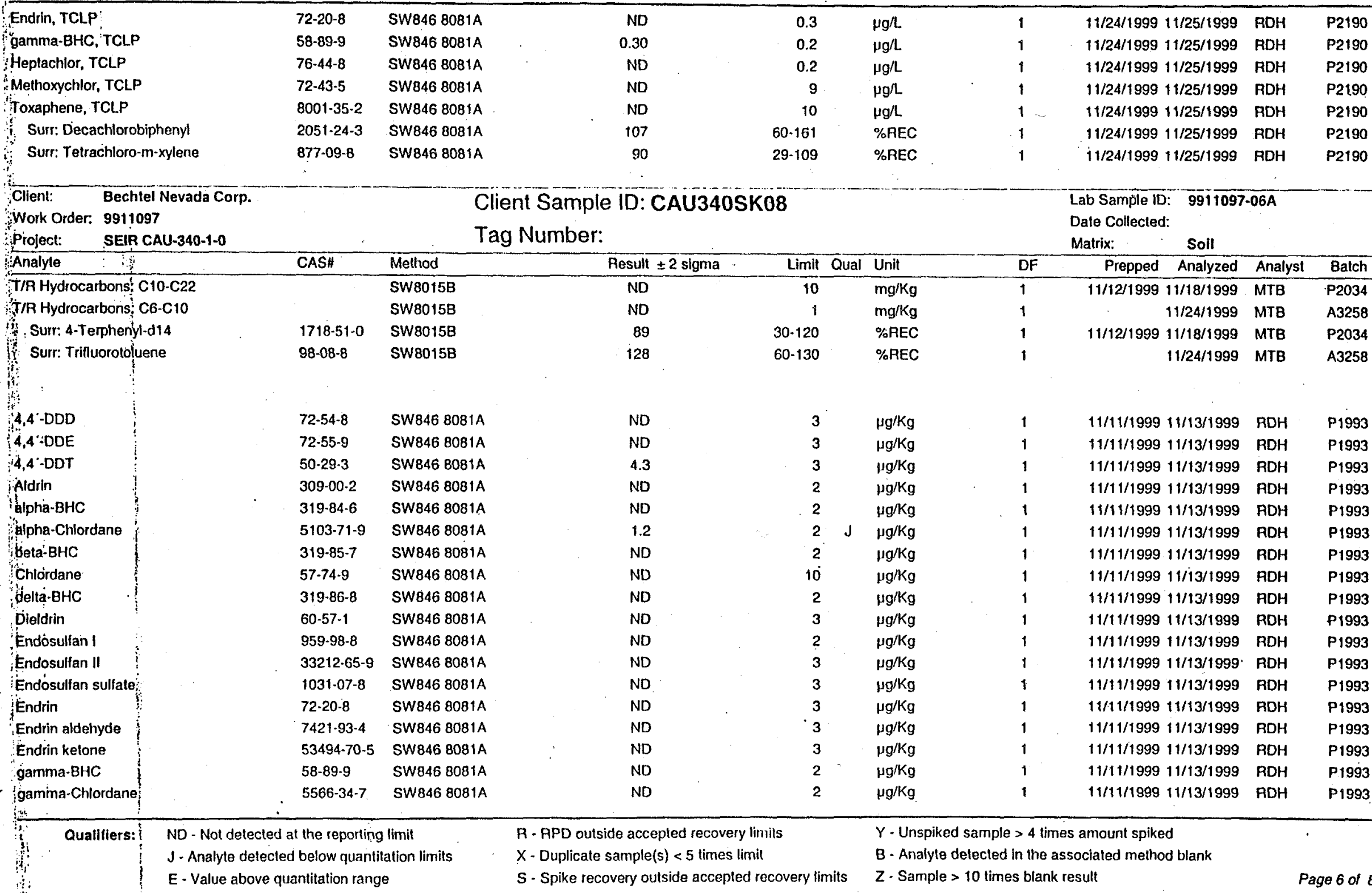


$\begin{array}{lllll}\text { IS000 W 6ih Avenue Suite } 300 & \text { Golden, Colorado } 80401-5047 & \text { (800) 6.54-0.506 } & \text { (303) 277-1687 Fax (303) 277.1689 }\end{array}$

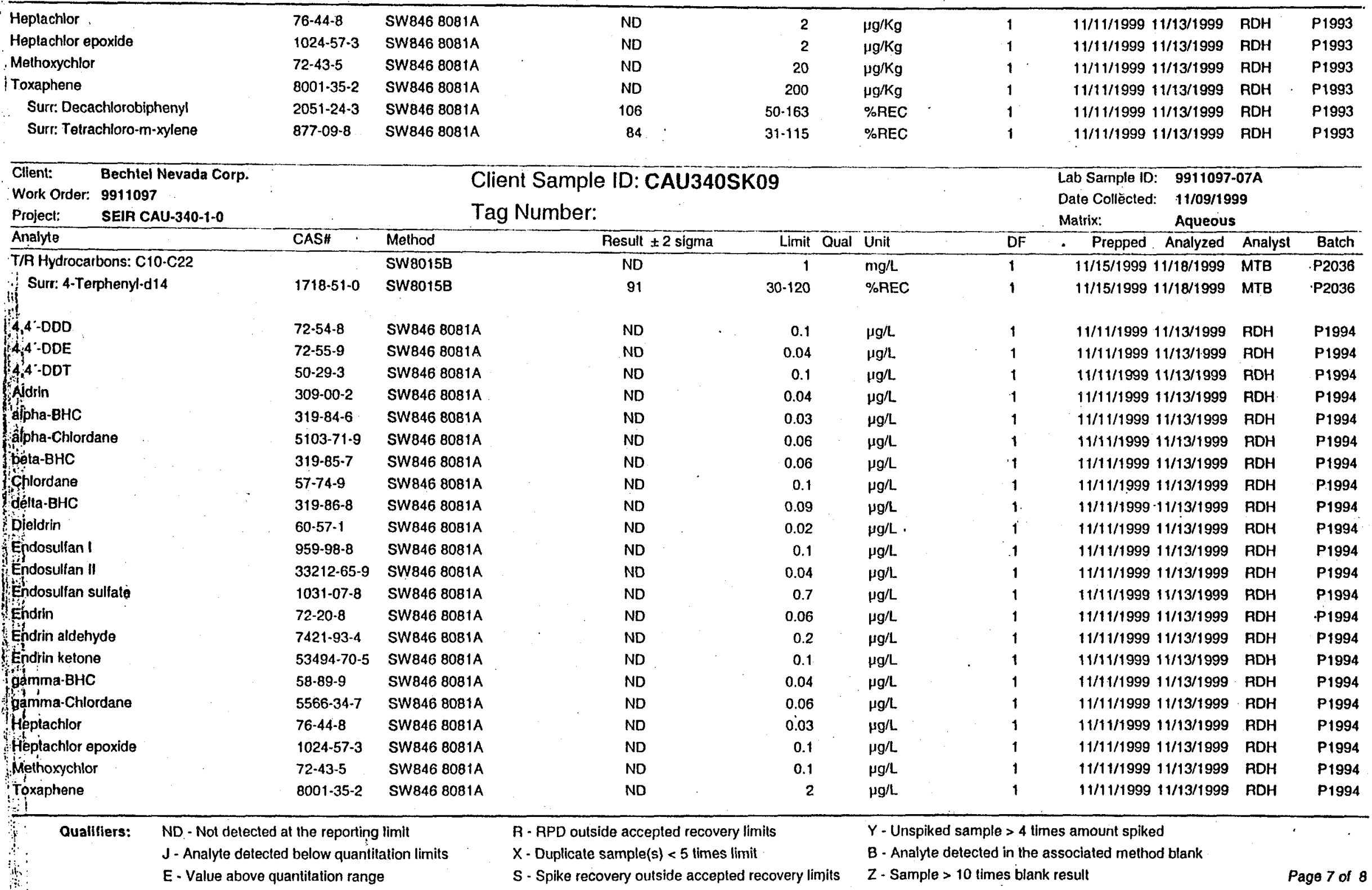



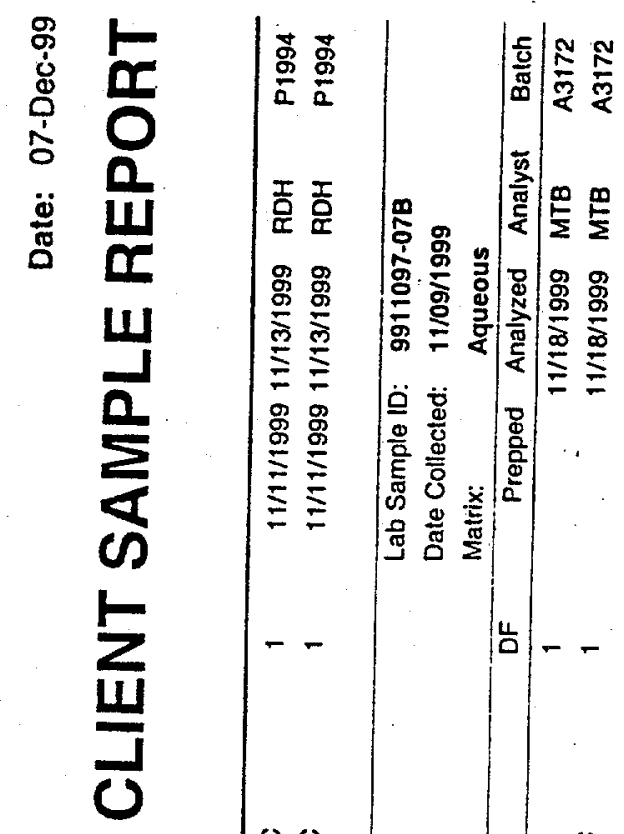
Date: $07-$ Dec-99

BATCH QC SUMMARY REPORT

I50ọo W6 6th Avemue Suite 300 Golden, Colurado 80401-5047 (800) 6.54-0.506 (303) 277-1687 Fax (.30.3) 277-1689

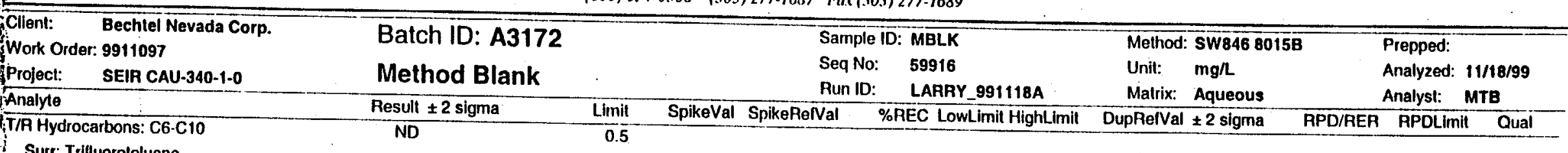

Surr: Trilluorotoluene

$100 \quad 60 \quad 130$

Client: Bechtel Nevada Corp.

Work Order: 9911097

Project: SEIR CAU-340-1-0

Analyte

Sample 1D: 9911097-07BMS

Seq No: " 59918

Melhod: SW846 8015B

Resull \pm 2 sigma

Run ID: LARRY_991118A

Matrix: Aqueous

Prepped: $\begin{array}{rr}\text { Limit } & \text { SpikeVal SpikeReIVa } \\ 0.5 & 2.0\end{array}$

LARRY_991118A

Analyzed: $11 / 18 / 99$

Surr: Trifluorololuene

2. 103

Analyst: MTB

.

Client: Bechlel Nevada Corp.

Work Order: 9911097

Batch ID: $\mathbf{A 3 1 7 2}$

100

70

130

Project: SEIR CAU-340-1-0

Matrix Spike Duplicate

Sample ID: 9911097-07BMSD

Seq No: 59919

130

$\begin{array}{lrrr}\text { Analyte } & \text { Result } \pm 2 \text { sigma } & \text { Limit } & \text { SpikeVal SpikeRelVa } \\ \text { T/A Hydrocarbons: } \text { C6.C10 } & 2.1 & 0.5 & 2.0\end{array}$

LARRY $991118 A$

Method: 5 SW846 8015B
Unit: mg/L
Matrix:

AEC Low Limil Highlimit DUpPIVal \pm 2 sigma

Surr: Trifluorololuene

$2.100 .5 \quad 2.0 \quad$ ND

al \%AEC

upReival \pm 2 sigma 107

100 70 130
130 2.1

Analyzed: $11 / 18 / 99$

$\therefore$

Client: Bechtel Nevada Corp.

Work Order: 9911097

Sample ID: MBLK

Seq No: $\quad 61513$

Run ID: LARRY_991123A

Melhod: Sw8015B

Unit: $\quad \mathbf{m g} / \mathrm{L}$

$\%$ LAREC LowLimil HighLimit DupRelVal \pm 2 sigma

Resull \pm 2 sigma Limil SpikeVal SpikeRerVa

Analyte ND

135

$60 \quad 130$

Analyzed: 11/23/99

Analyst: MTB

i Surr: Trifluorololuene

Client: Bechtel Nevada Corp.

Work Order: 9911097

Project: SEIR CAU-340-1-0

Batch ID: $\mathbf{A 3 2 5 8}$

9911097-01AMS

130

Resull \pm 2 sigma

$\begin{array}{ll}\text { Sample ID: 9911097-01AMS } & \text { Method: SW8015B } \\ \text { Seq No: } 61515 & \text { Unit: } \mathrm{mg} / \mathrm{Kg}\end{array}$

Run ID: LARRY_991123A Matrix: Soll

Malrix: Soll

APD/RER APDLimit Qual

T/R Hydrocarbons: C6-C10

1.3

DupRefVal \pm 2 sigma

Prepped:

Analyzed: 11/23/99

Analyst: MTB

Surr: Trilluorotoluene $84 \quad 70 \quad 130$

$103 \quad 60 \quad 130$

Quallfiers: ND - Not detected at the reporting limit

J - Analyte detecled below quantitation limits

$\mathrm{E}$ - Value above quantitation range
R - APD outside accepted recovery limits

$X$ - Duplicale sample(s) $<5$ times limit

$S$ - Spike recovery outside accepled recovery limits
$Y$ - Unspiked sample $>4$ times amount spiked

$B$ - Analyle delected in the associated method blank

$Z$ - Sample $>10$ times blank resull 
Date: $07-$ Dec-99 Barringer Laboratories, Inc.

BATCH QC SUMMARY REPORT

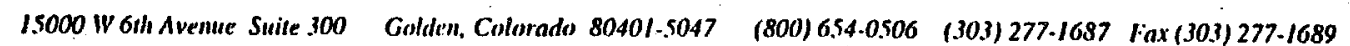

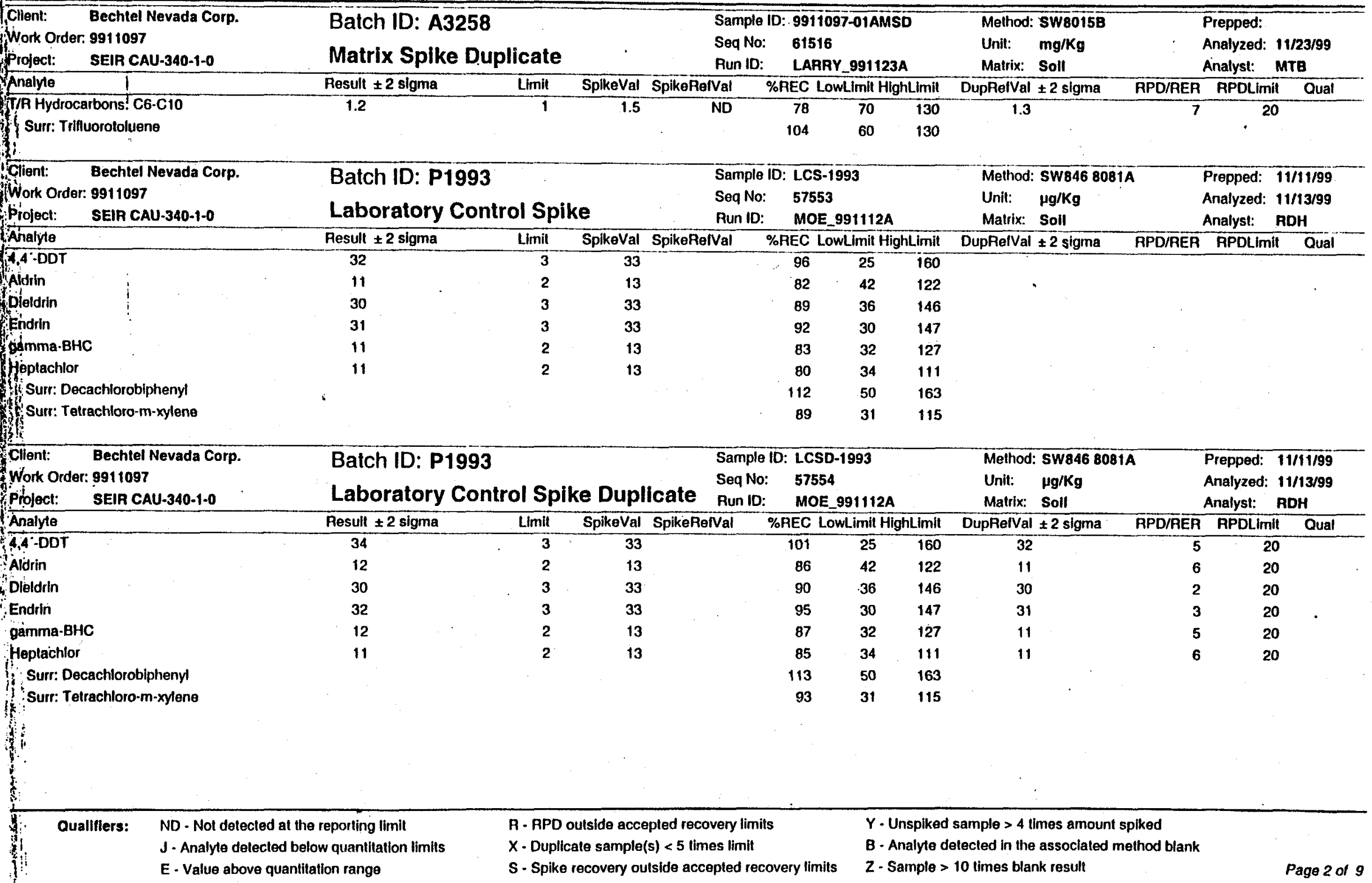

E. Value above quantitalion range

S - Spike recovery outside accepted recovery limils $Z$ - Sample $>10$ limes blank result 
Date: $07-$ Dec-99 Barringer Laboratories, Inc.

\section{BATCH QC SUMMARY REPORT}

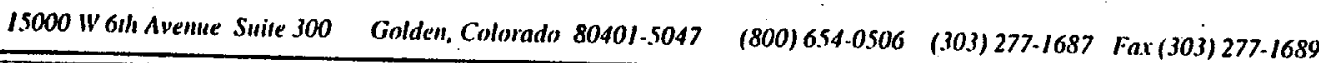

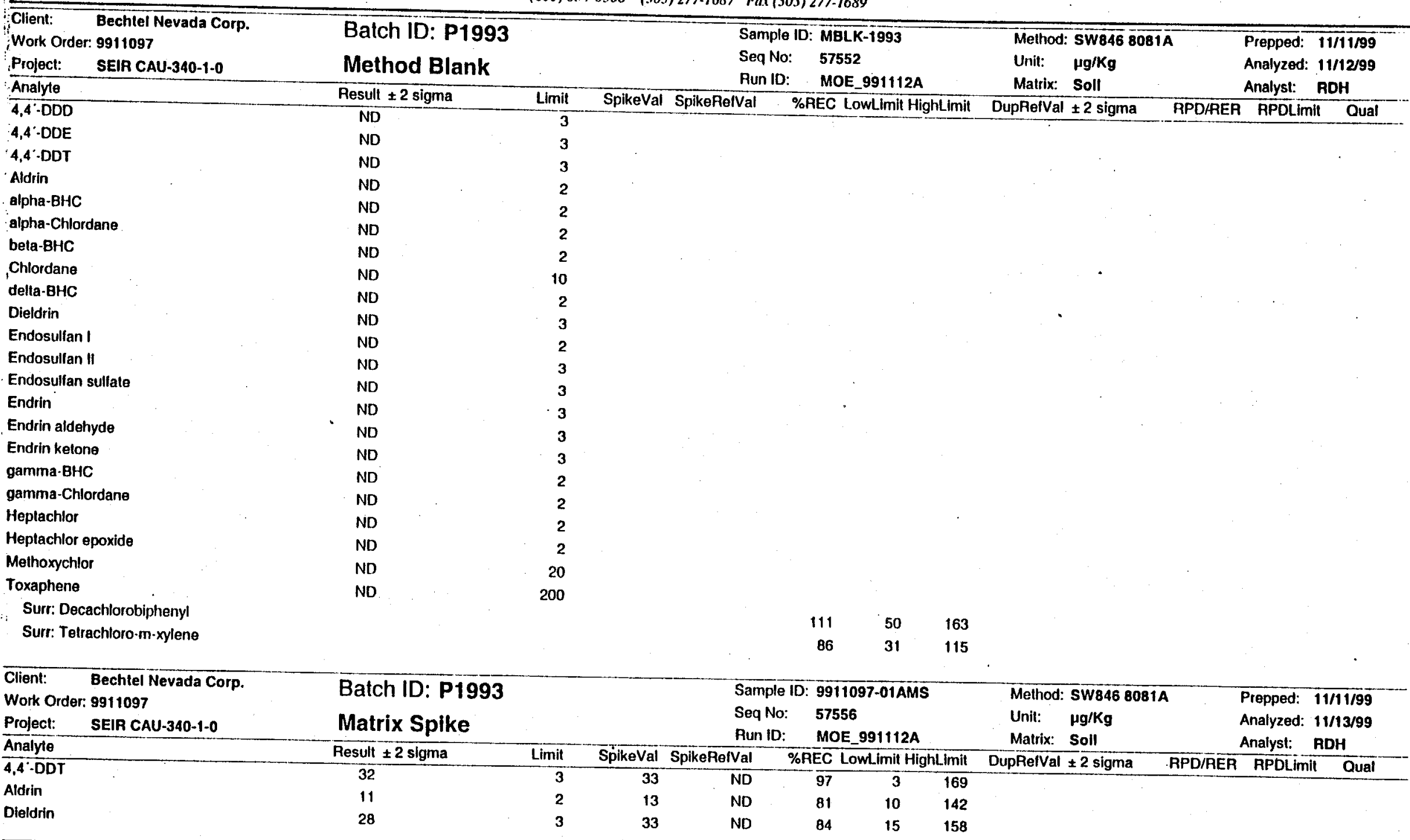

Qualliters:

ND - Not detecled at the reporting limi

$\mathrm{J}$ - Analyte detected below quantilation limits

A - RPD outside accepted recovery limils

$Y$ - Unspiked sample $>4$ times amount spiked

E - Value above quantitation range

$X$ - Düplicate sample(s) $<5$ times limit

$B$ - Analyte detected in the assoclated method blank

S. Spike recovery outside accepled recovery limits $\quad Z$ - Sample $>10$ times blank result 
Date: $07-$ Dec-99 Barringer Laboratories, Inc.

BATCH QC SUMMARY REPORT

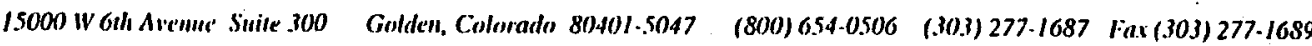

\begin{tabular}{|c|c|c|c|c|c|c|}
\hline Endrin & 29 & 3 & 33 & ND & 88 & 20 \\
\hline gamma-BHC & 11 & 2 & 13 & ND & 81 & 30 \\
\hline Heptachlor & 11 & 2 & 13 & ND & 79 & 18 \\
\hline Surr: Decachlorobiphenyl & & & . & & 110 & 50 \\
\hline Surr: Tetrachloro-m-xylene & & & & & 89 & 31 \\
\hline
\end{tabular}

\begin{tabular}{|c|c|c|c|c|c|c|c|c|c|c|c|c|c|}
\hline \multirow{2}{*}{\multicolumn{2}{|c|}{ 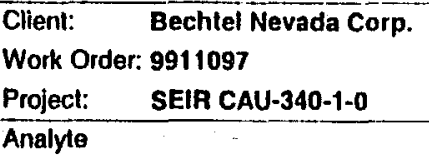 }} & \multicolumn{2}{|c|}{$\begin{array}{l}\text { Batch ID: P1993 } \\
\text { Matrix Spike Duplicate }\end{array}$} & \multirow[b]{2}{*}{ SpikeVal } & \multicolumn{4}{|c|}{$\begin{array}{ll}\text { Sample ID: } & 9911097-01 \text { AMSD } \\
\text { Seq No: } & 57557 \\
\text { Run ID: } & \text { MOE_991112A }\end{array}$} & \multicolumn{3}{|c|}{$\begin{array}{l}\text { Melhod: SWB46 8081A } \\
\text { Unit: } \quad \mu g / K g \\
\text { Matrix: Soll }\end{array}$} & \multicolumn{2}{|c|}{$\begin{array}{l}\text { Prepped: } 11 / 11 / 99 \\
\text { Analyzed: } 11 / 13 / 99 \\
\text { Analyst: } \text { RDH }\end{array}$} \\
\hline & & Result \pm 2 sigma & Limit & & SpikeRefval & \%REC & LowLimit & Limit & DupReIVal & \pm 2 sigma & RPD/RER & APDL imit & Qual \\
\hline $4,4^{\circ} \cdot \mathrm{DDT}$ & & 27 & 3 & 33 & ND & 81 & 3 & 169 & 32 & . & 19 & 20 & 0 \\
\hline Aldrin & & 9.3 & 2 & 13 & ND & 70 & 10 & 142 & 11 & & 15 & 20 & 0 \\
\hline Dieldrin & & 24 & 3 & 33 & ND & 72 & 15 & 158 & 28 & & 15 & 20 & 0 \\
\hline Endrin & & 25 & 3 & 33 & ND & 75 & 20 & 112 & 29 & & 16 & 20 & 0 \\
\hline gamma-BHC & & 9.4 & 2 & 13 & ND & 71 & 30 & 114 & 11 & & 14 & 20 & 0 \\
\hline Heplachlor & & 9.5 & 2 & 13 & ND & 72 & 18 & 141 & 11 & & 10 & 20 & 0 \\
\hline Surr: Dece & achlorobiphenyl & & & & & 101 & 50 & 163 & & & & & \\
\hline Surr: Tetre & achloro-m-xylene & & & & & 80 & 31 & 115 & & & & & \\
\hline
\end{tabular}

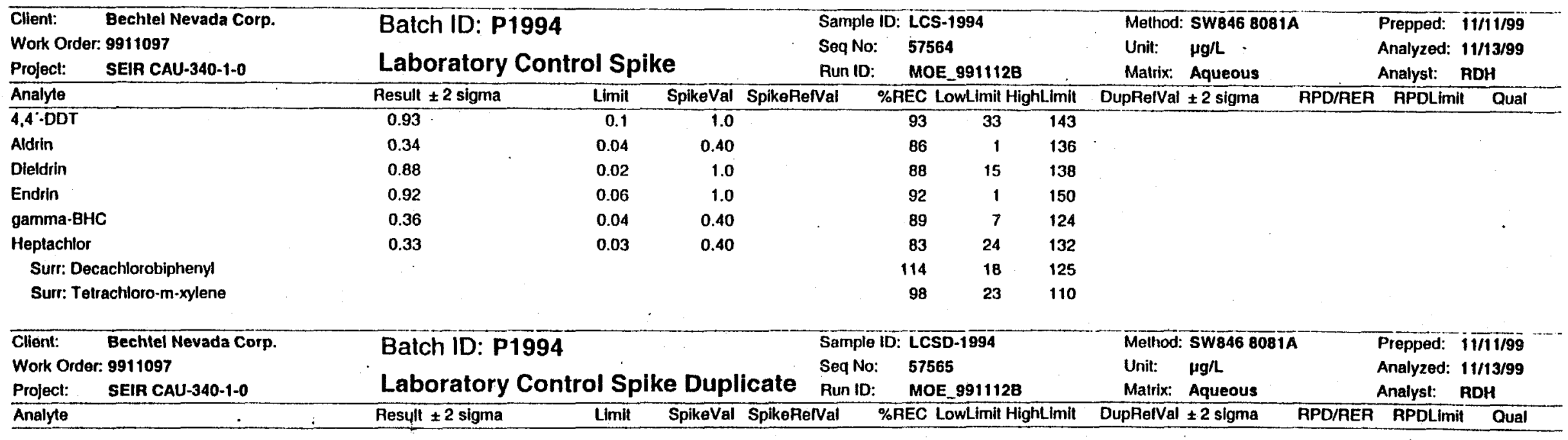

$\begin{array}{ll}\text { Qualiflers: } & \text { ND - Not detected at the reporting limit } \\ & \mathrm{J} \text { - Analyte detected below quantitation limits } \\ & \text { E - Value above quantilation range }\end{array}$

R - RPD outside accepted recovery limils

$X$ - Duplicate sample(s) $<5$ limes limil

S - Spike recovery outside accepted recovery limits
$Y$ - Unspiked sample > 4 times amount spiked

$B$ - Analyte delected in the associated method blank

$Z$ - Sample $>10$ times blank result 
Date: $07-$ Dec-99 Barringer Laboratories, Inc.

BATCH QC SUMMARY REPORT

15000 W 6ilh Avenue Stuite 300 Golden, Colorado 80401-5047 (800) 054.0506 (303) 277-1687 Fax (303) 277.1689

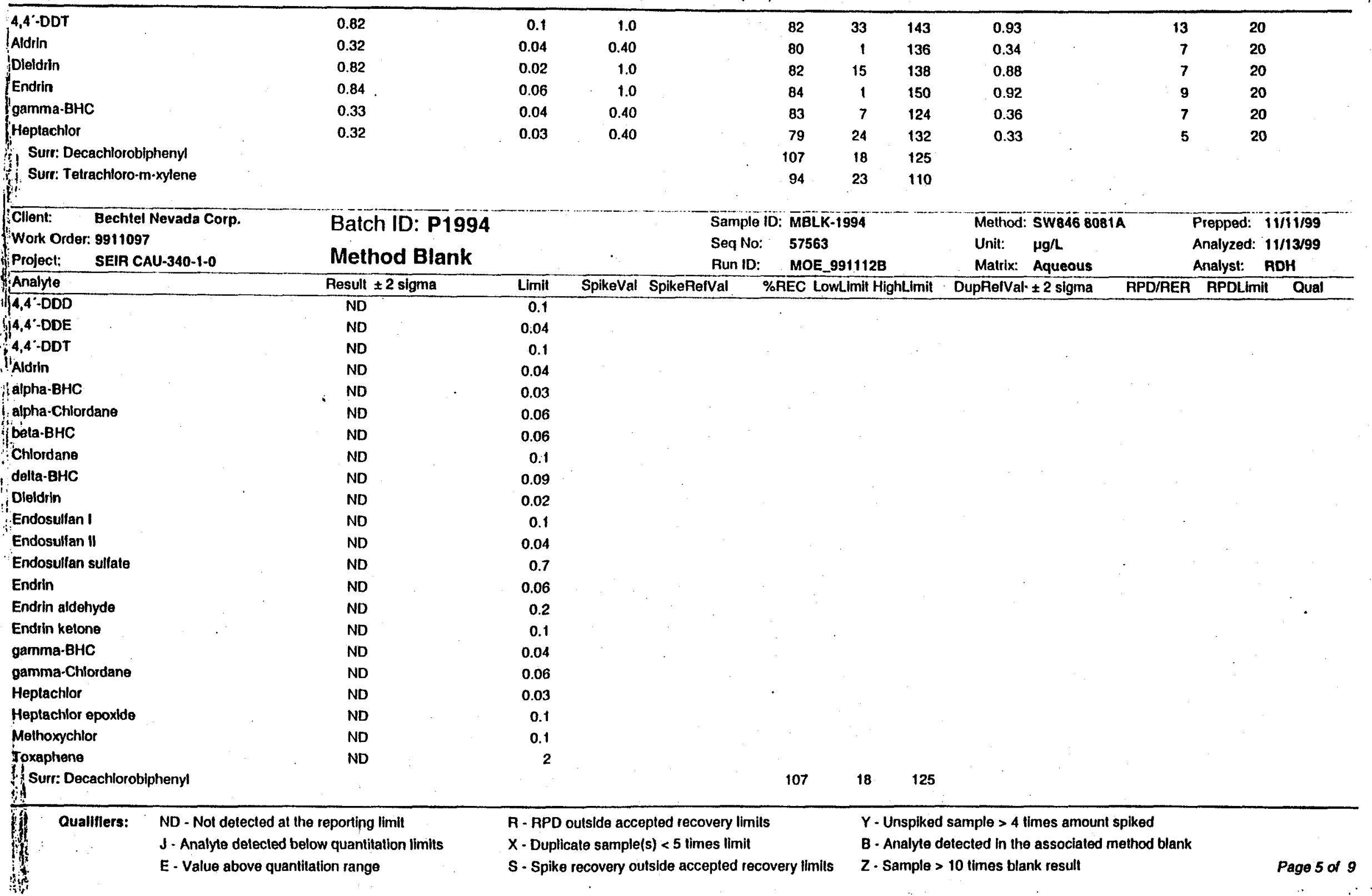


Date: $07-$ Dec-99

BATCH QC SUMMARY REPORT

1.5000 W Gih Avenue Suite 300 Golden, Coltirado 80401-5047

(800) 6.54-0.506 (.30.3) 277-1687 $\operatorname{Far}(30.3) 277.1689$

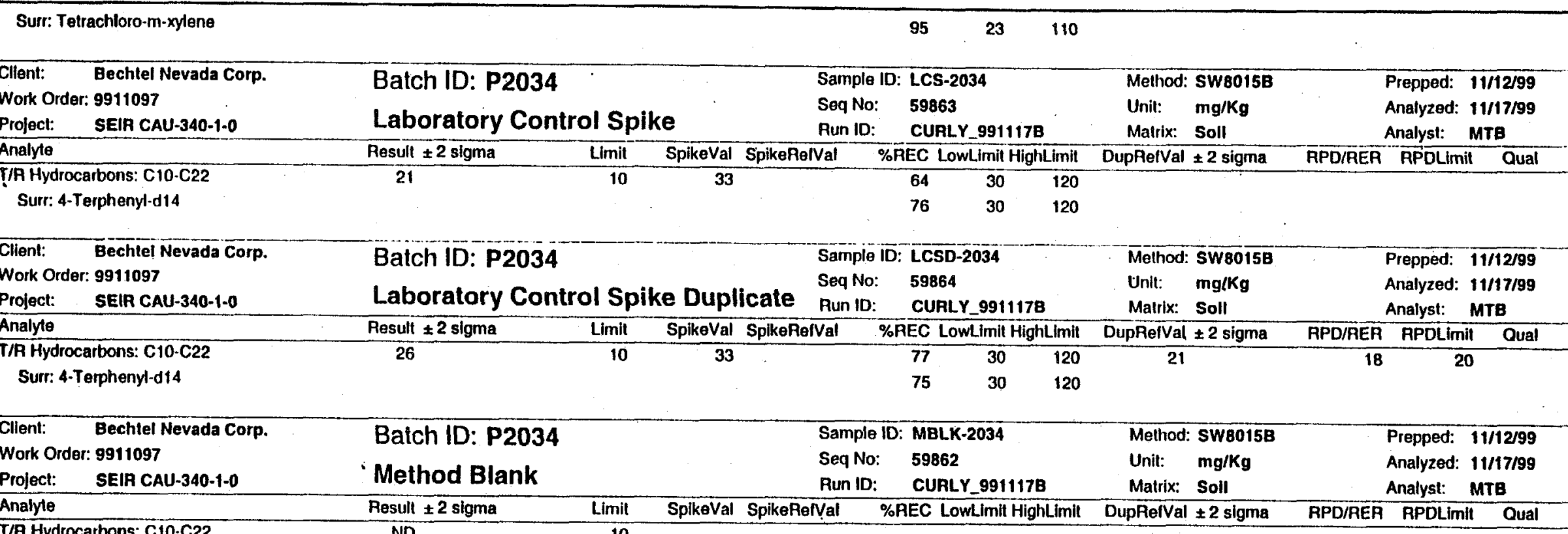

T/A Hydrocarbons: C10.C22

ND

$\begin{array}{lll}73 & 30 \quad 120\end{array}$

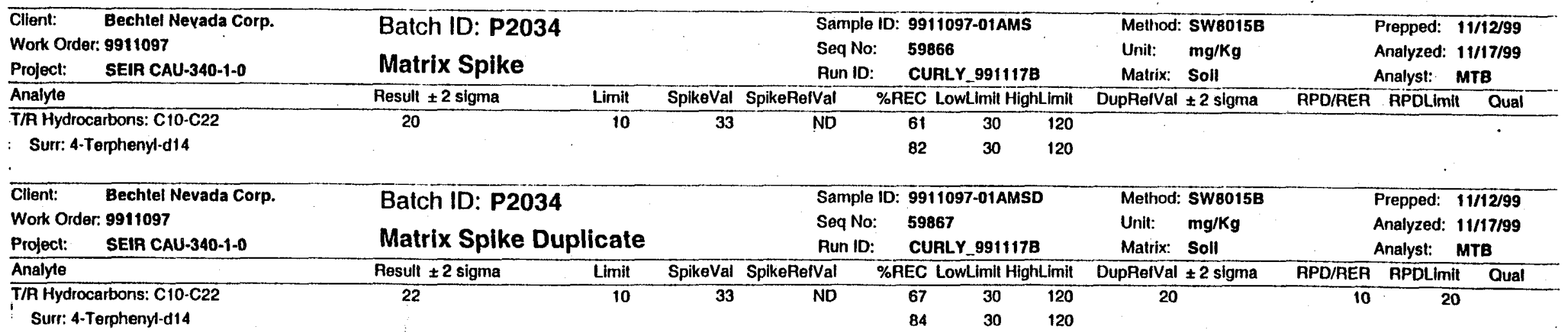

Surr: 4-Terphenyl-d14

R - RPD outside accepted recovery limits

$X$ - Duplicale sample(s) $<5$ times limit

S - Spike recovery outside accepted recovery limits
$Y$ - Unsplked sample $>4$ limes amount spiked

$B$ - Analyle delected in the associated method blank

Z - Sample $>10$ times blank result
$\mathrm{J}$ - Analyle delecled below quantitalion limis

E - Value above quantitation range iti; ; 
Date: 07-Dec-99 Barringer Laboratories, Inc.

BATCH QC SUMMARY REPORT

I5000 W Gih Avenue Suive 300 Golden, Colorado 80401-5047 (800)654-0506 (303) 277-1687 Fax (303) 277.1689

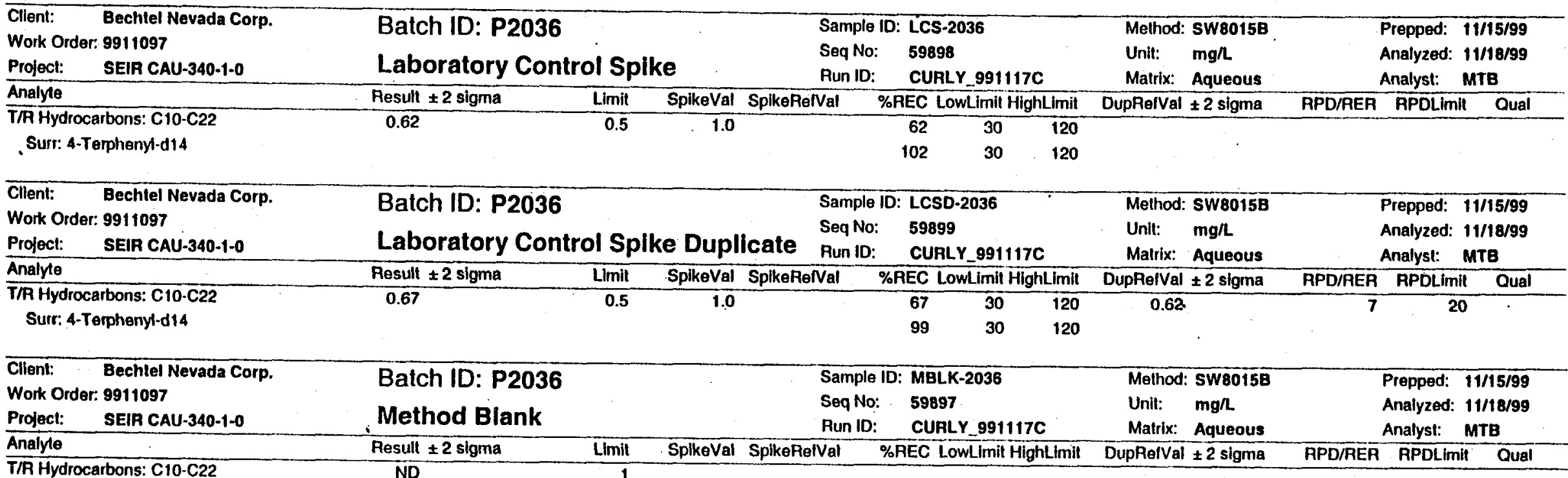

T/A Hydrocarbons: C10-C.22 ND

$98 \quad 30 \quad 120$

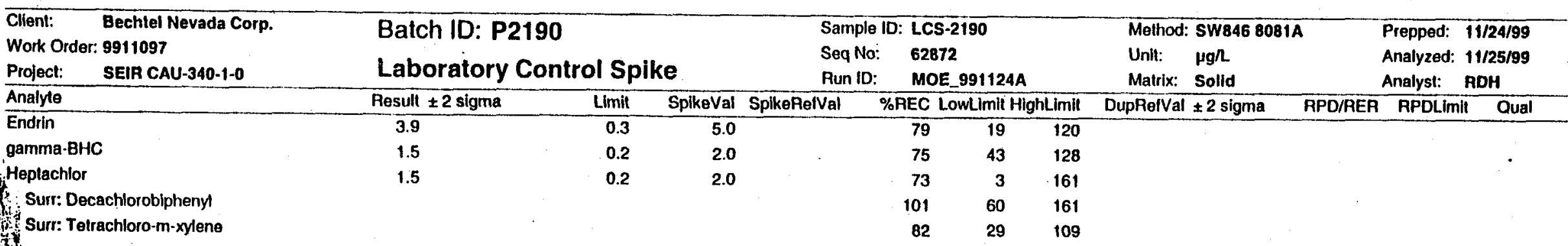

Surr: Tetrachloro-m-xyen

Client: Bechtel Nevada Corp.

Work Order: 9911097

Projoct: SEIR CAU-340-1-0

Batch ID: P2190

Sample 10; LCSD-2190

Laboratory Control Spike Duplicate Seq No: 62873

Method: SW846 8081A

Prepped: 11/24/99

Analyte SEIR CAU-340-1-0

Result +2 sigma Limit SpikeVal SpikePo(va

ID: MOE_991124A

$\quad \mu \mathrm{g} / \mathbf{L}$

Analyzed: $11 / 25 / 99$

Analyst: RDH

Quallilers:

ND - Nol detected at the reporting limit

R - RPD outside accepted recovery limits

$Y$. Unsplked sample $>4$ times amount spiked

J. Analyte detected below quantitalion limits

$X$ - Duplicate sample $(s)<5$ times limil

$B$ - Analyte detected in the associated method blank

E - Value above quantitation range

$S$ - Spike recovery oulside accepled recovery limits

$Z$ - Sample $>10$ limes blank resull 
Date: 07-Dec-99

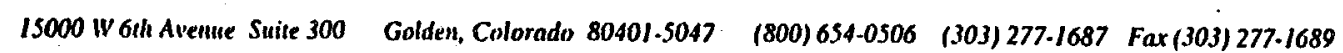

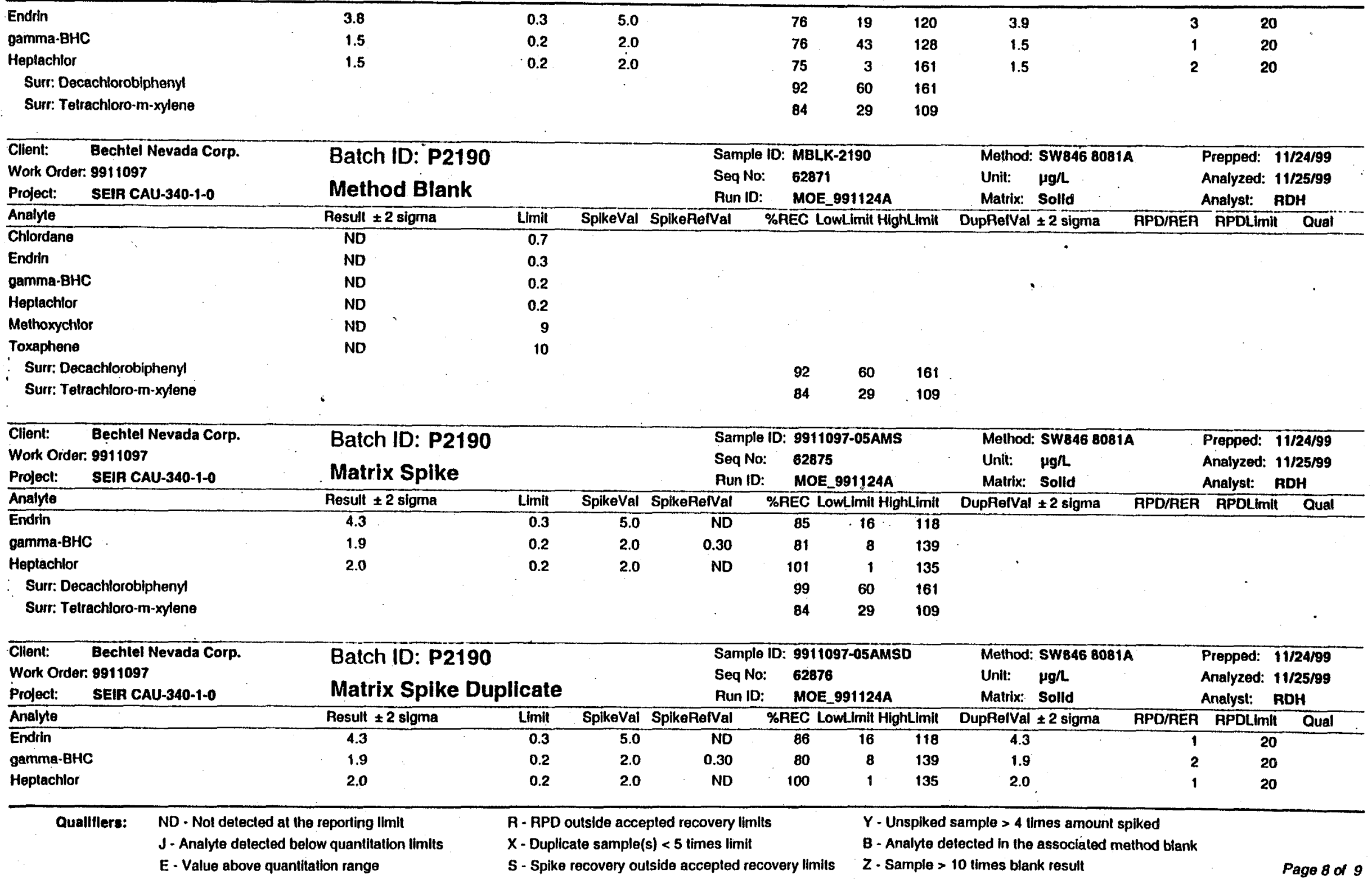

E. Value above quantilation range

$(s)<5$ times limit

B - Analyte delected in the associaled
$Z$ - Sample $>10$ times blank result 


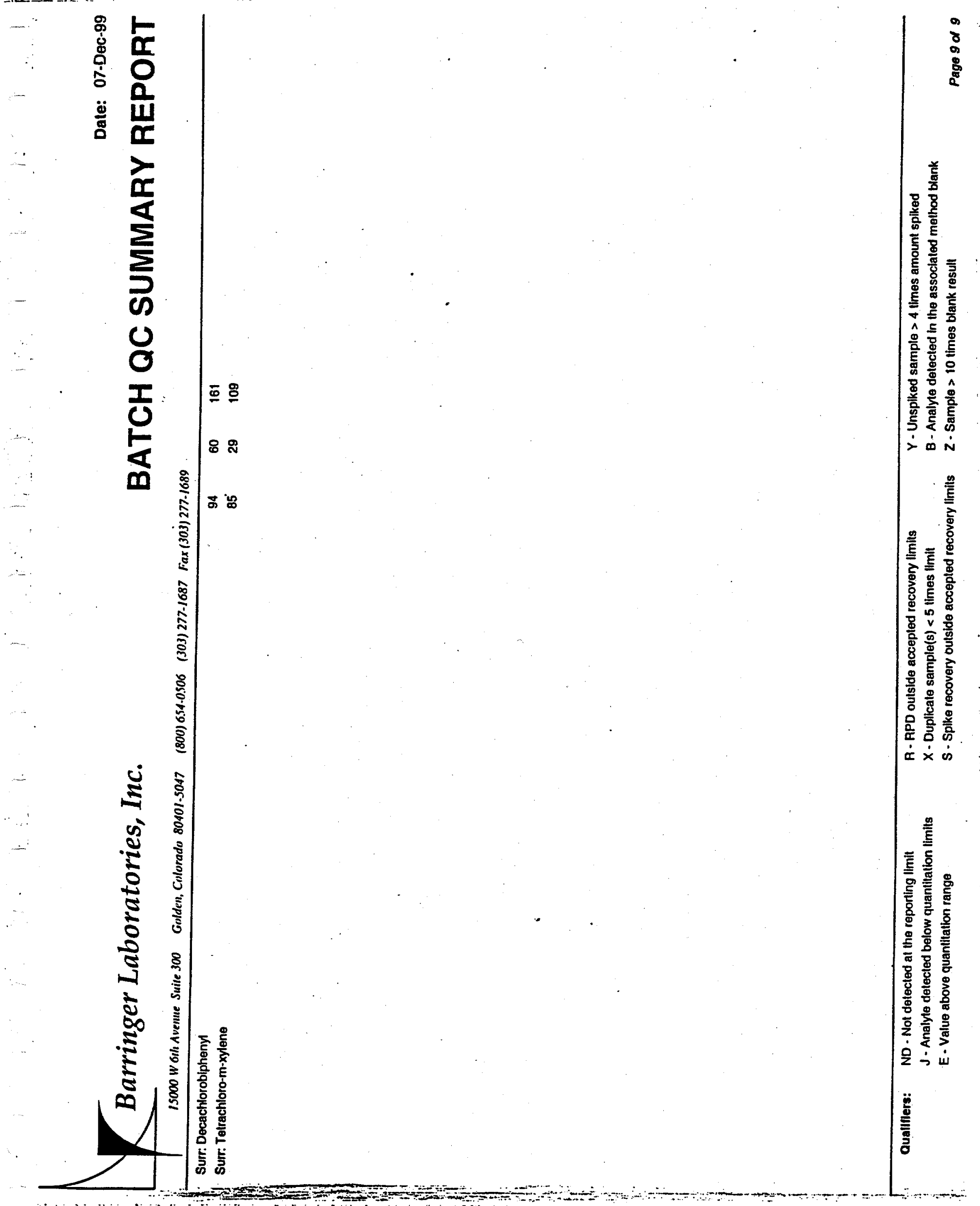



Job Number: 23220

Facility ID: Ars

Sampling Event: cNu 340

COC Number: cav 340-1
Cooler 10:

Cooter Temp:

Number of Coolers:
Dellvery Order 10:

SEIR No.: CN 340-1-0

Charge Code: Cooo24an

Laboratory: BARImoR

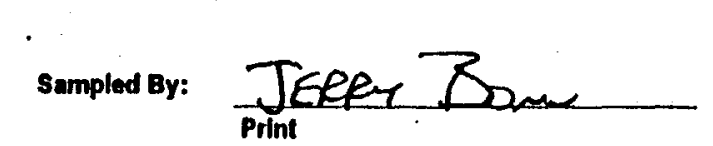

Requested Analysis:

Cooler Units:

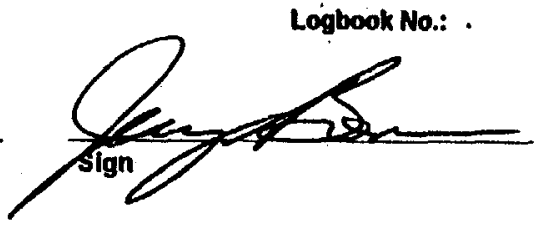

Print

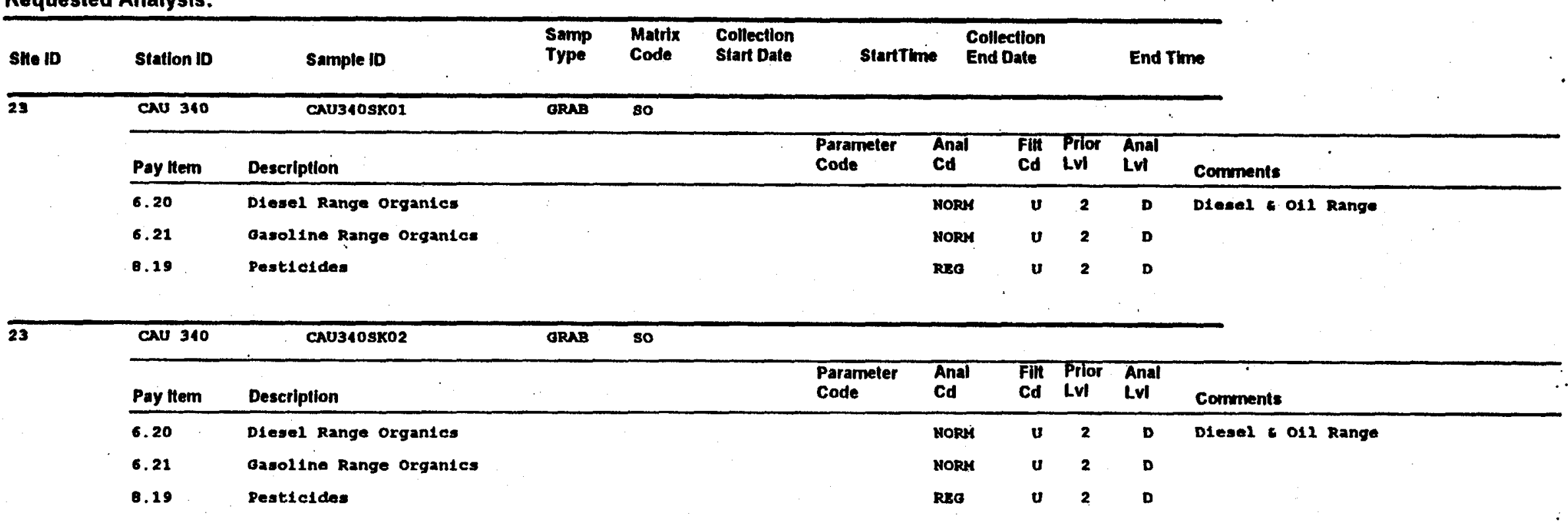

This package conforms to the conditions and limitalions speciried in 49 CFR 173.421 for excepled radioactive malerlal, llmited quantity, n.0.8., UN2910 
Requested Analysis:

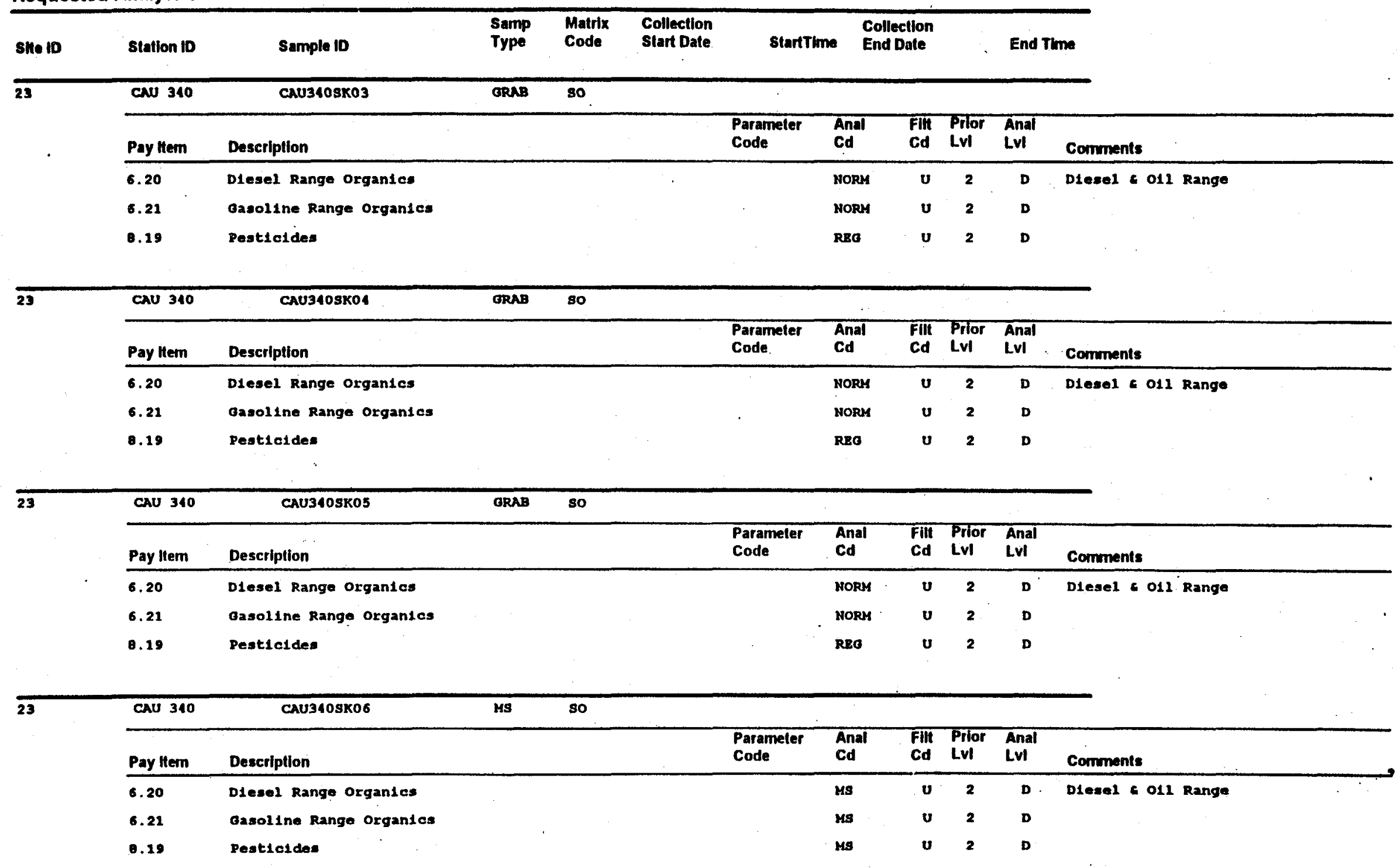

Thls package conforms to the conditions and limitations speciried in 49 CFR 173.421 for excepted radioactive material, IImiled quantity, n.0.8., UN2910. 


\section{Requested Analysis:}

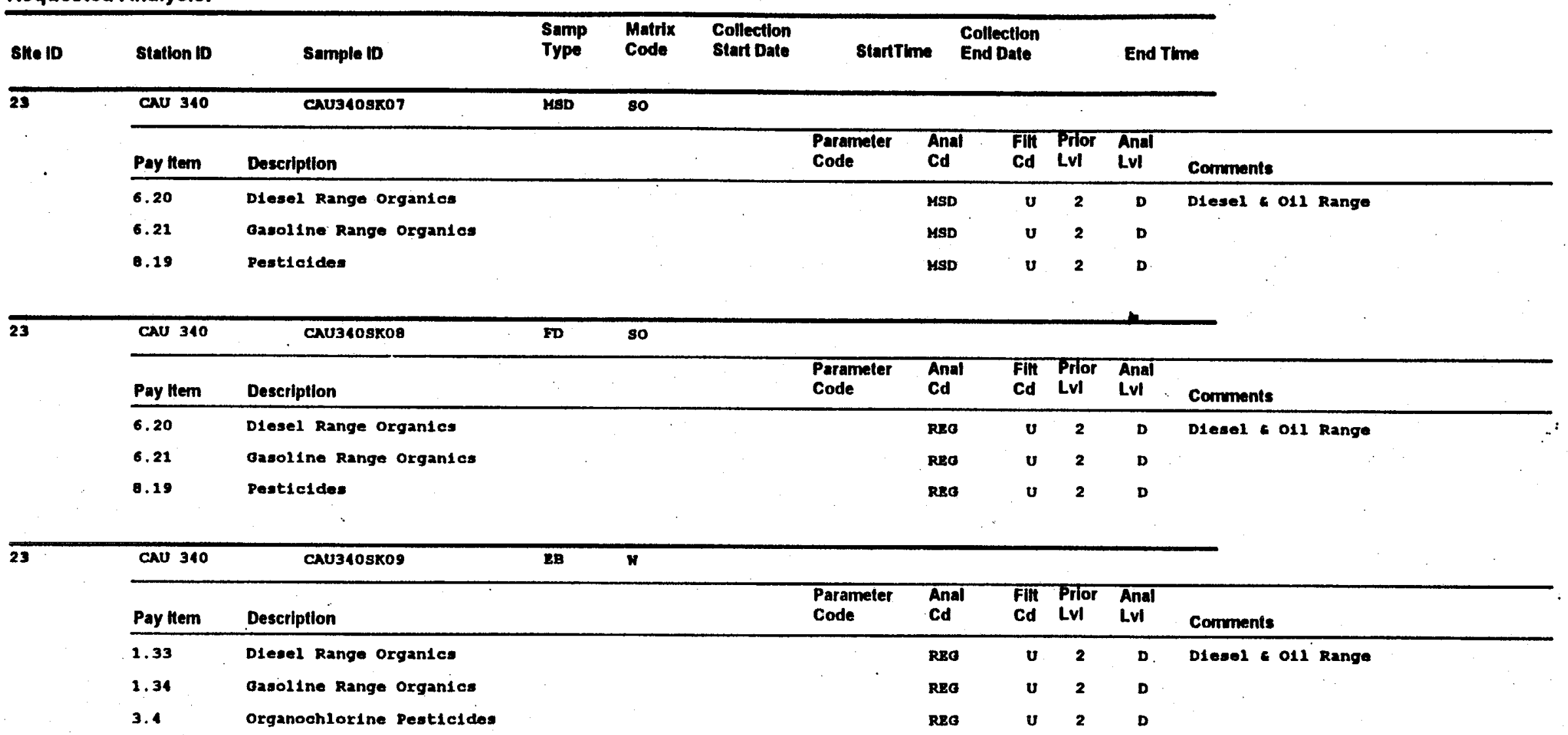




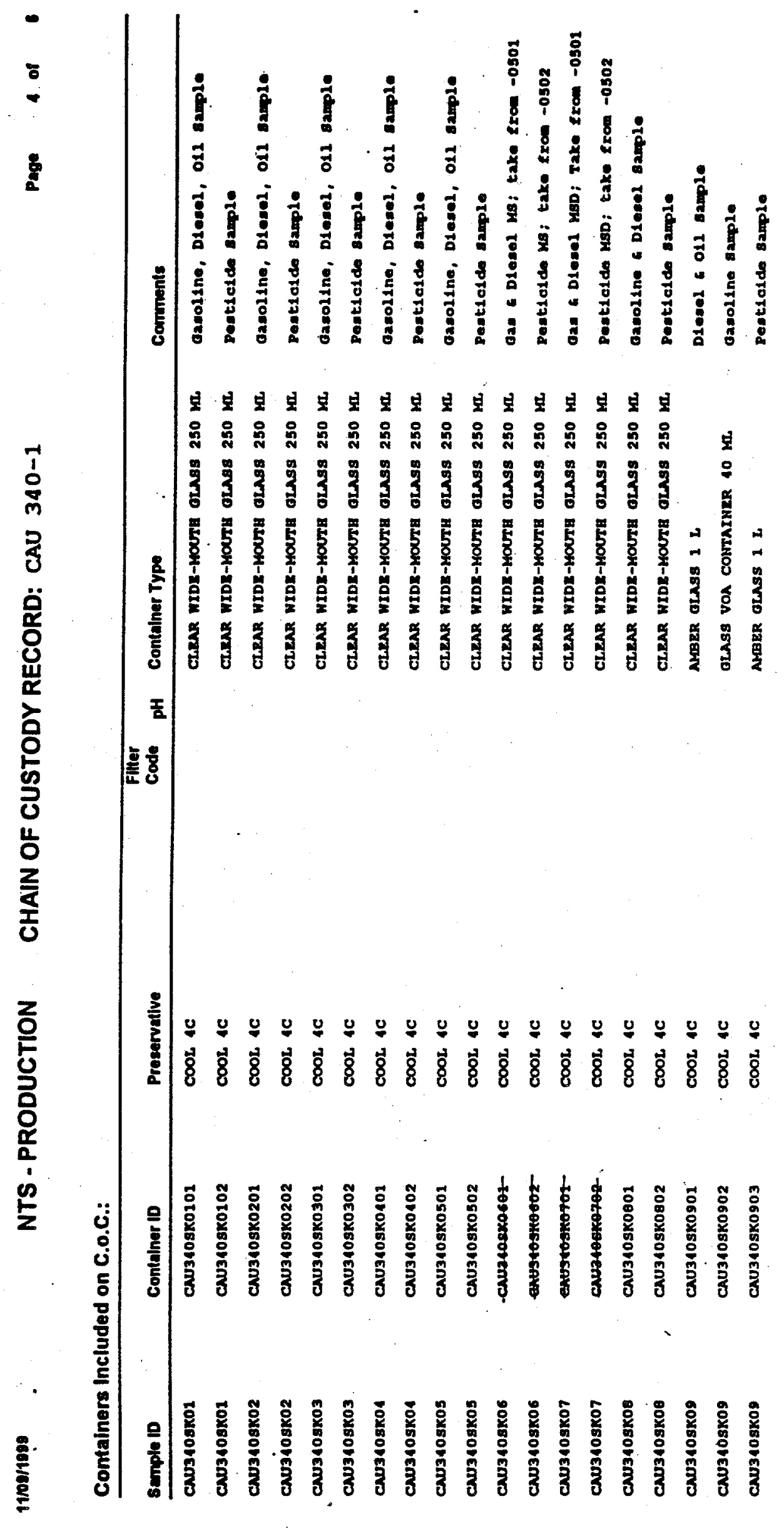


Tranafor Information:

$$
\text { Iolmmuvray Ullera } 10.00 \text { Receipt by } L A B
$$

\section{Comments:}

Th1e coc generated for $J$. Bonn

\section{Potential Contamination Yes No}

Radiological

Chemical 

Barringer Laboratories, Inc.

15000 W 6ri Avenue Suise 300 Golden, Colorado 80401-5047 (800) 654-0506 (303) 277.1687 Far (30.3) 277-1689

Ted Redding

Bechtel Nevada Corp.

P.O. Box 98521

MS NTS 273

Las Vegas, NV 89193-8521 .

Phone: $1-702-295-7220$

Fax: $1-702-295-4773$

Work Order: 9911141

Project: 23220/ SDG\#:V721

Dear Ted Redding,

Barringer Laboratories received 15 samples on $11 / 17 / 99$ for the analyses presented in the following report.

There were no problems with the analyses and all data for associated QC met EPA or laboratory specifications except where noted in the Case Narrative.

If you have any questions regarding these test results, please feel free to call.

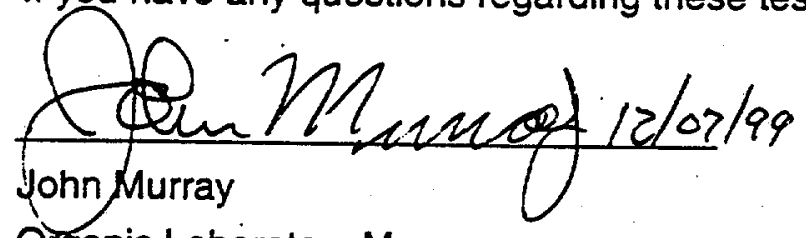

Organic Laboratory Manager

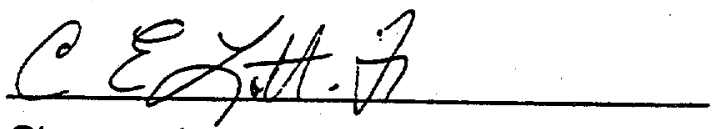

Project Review $12 / 7 / 99$ 

Date: $07-$ Dec-99

\section{Barringer Laboratories, Inc.}

15000.W 6th Avenue Suite $300 \quad$ Golden, Colorado 80401-5047 (800)654-0506 (303) 277.1687 Fax (303) 277.1689

\begin{tabular}{ll}
\hline Client: & Bechtel Nevada Corp. \\
Project: & $23220 /$ SDG\#:V721 \\
Work Order: & 9911141
\end{tabular}

All reported values in this report have been rounded to the correct number of significant figures. All calculations have been performed before applying significant figures, therefore, not all calculations may be reproducible with the results printed in this report.

Analytical Comments for METHOD SW846 8015BGRO, SAMPLE MBLK: The surrogate exceeded the set QC limit for the method blank which was non-detect for gasoline. Because the surrogate was high we believe we could have detected gasoline if present and therefore consider the data to be valid as reported.

Analytical Comments for method SW8015BGRO, sample 9911141-02A: The surrogate exceeded the set QC limit for this sample which was non-detect for gasoline. Because the surrogate was high we beleive we could have detected gasoline if present in the sample and therefore we consider the data to be valid as reported.

Analytical Comments for method SW846 8081A: There are some analytes that are being reported as estimated due to the analyte being above our calibration curve. The client is aware of this and does not require the samples analyzed at the required dilution to bring all analytes within the calibration range.

This report has been amended from the report dated $11 / 30 / 1999$ to include the dilutions of the samples that were previously reported with estimated results for method SW846-8081A. 
Date: $07-$ Dec-99

\section{Barringer Laboratories, Inc.}

$\begin{array}{lllll}15000 \text { W 6ih Avenue Suite } 300 \quad \text { Golden. Colorado 80401-5047 } & \text { (800) 6.54-0506 (303) 277.1687 Fax (303) 277-1689 }\end{array}$

Client: Bechtel Nevada Corp.

Project: 23220/ SDG\#:V721

Work Order: 9911141

SAMPLE SUMMARY

Date Received: $11 / 17 / 99$

Temp Received: $6^{\circ} \mathrm{C}$

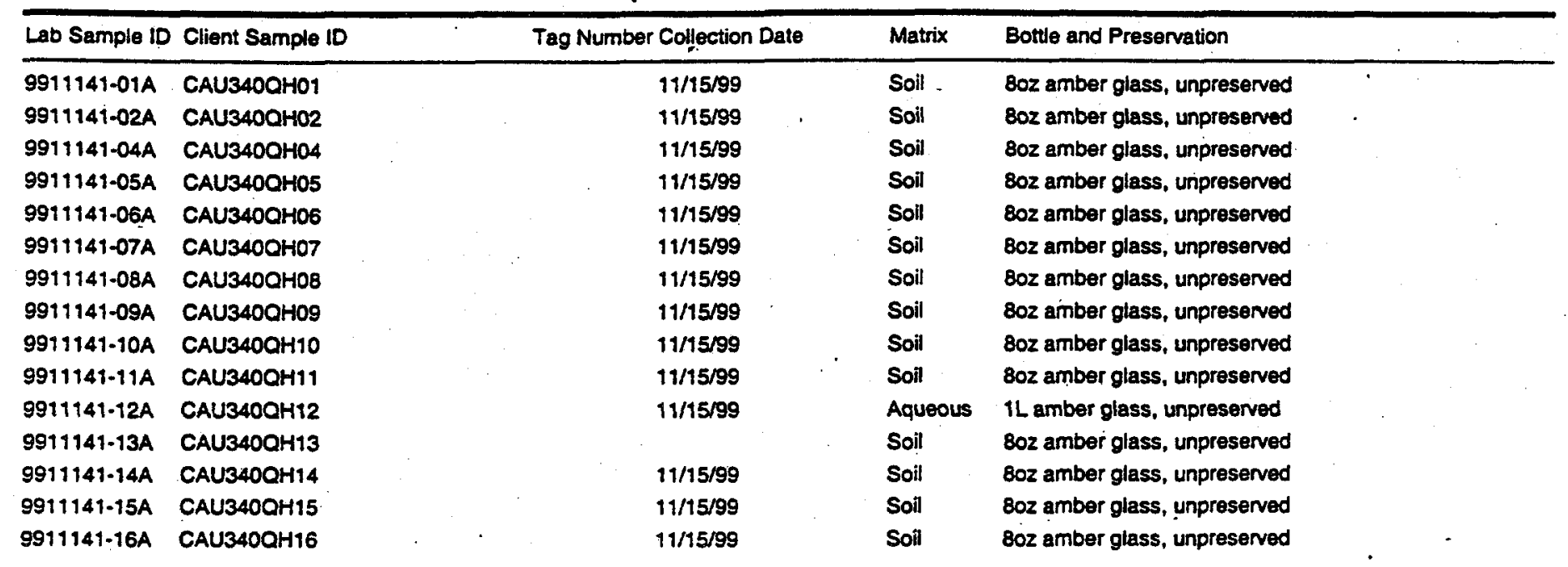


Date: 07-Dec-99 Barringer Laboratories, Inc.

\section{CLIENT SAMPLE REPORT}

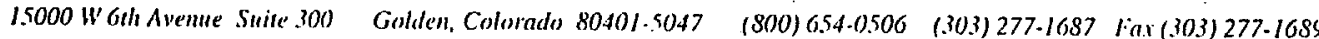

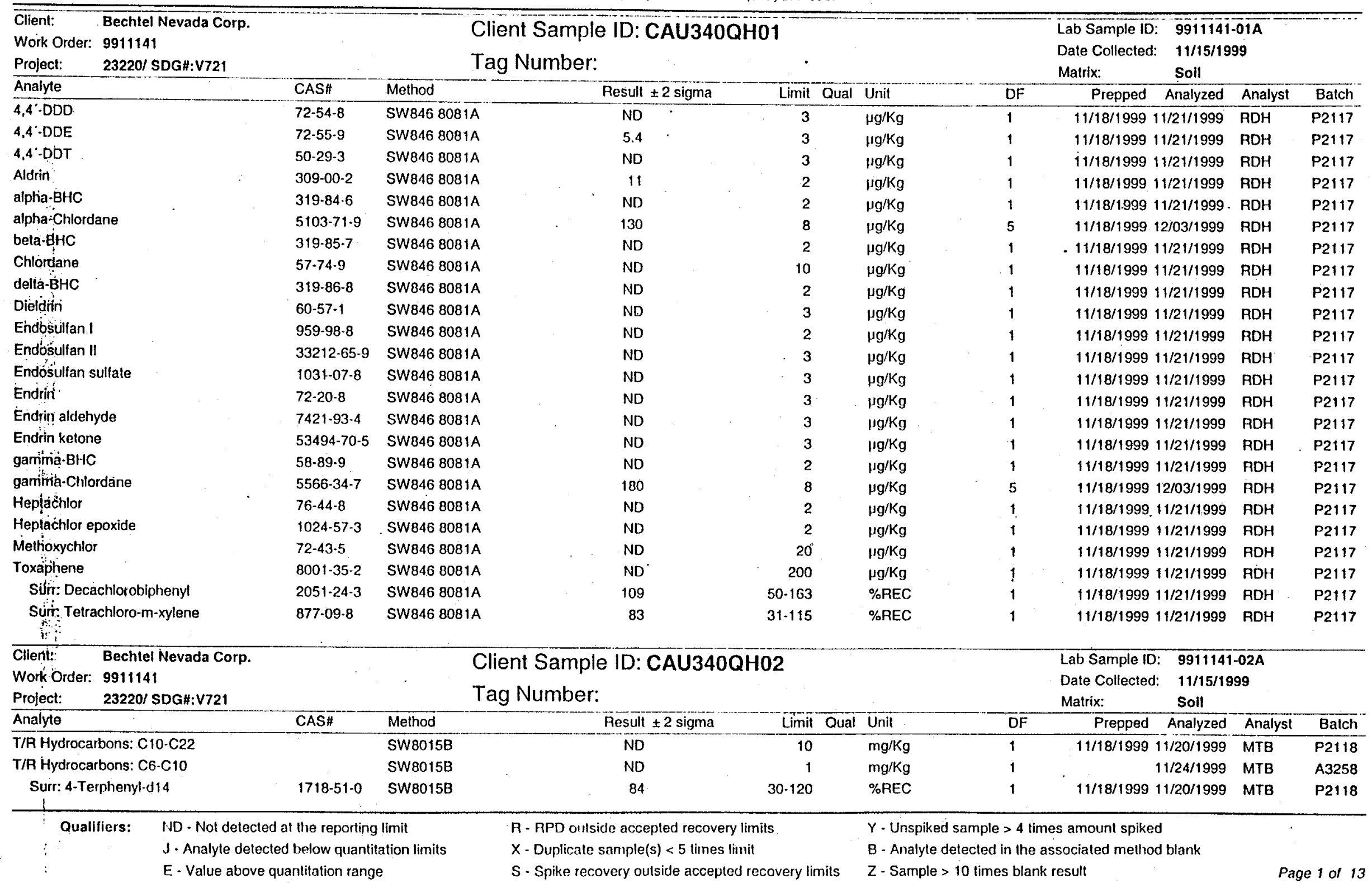


Date: $07-$ Dec-99

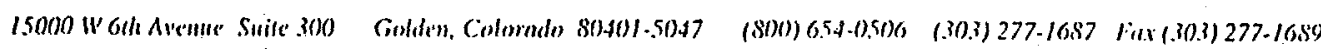

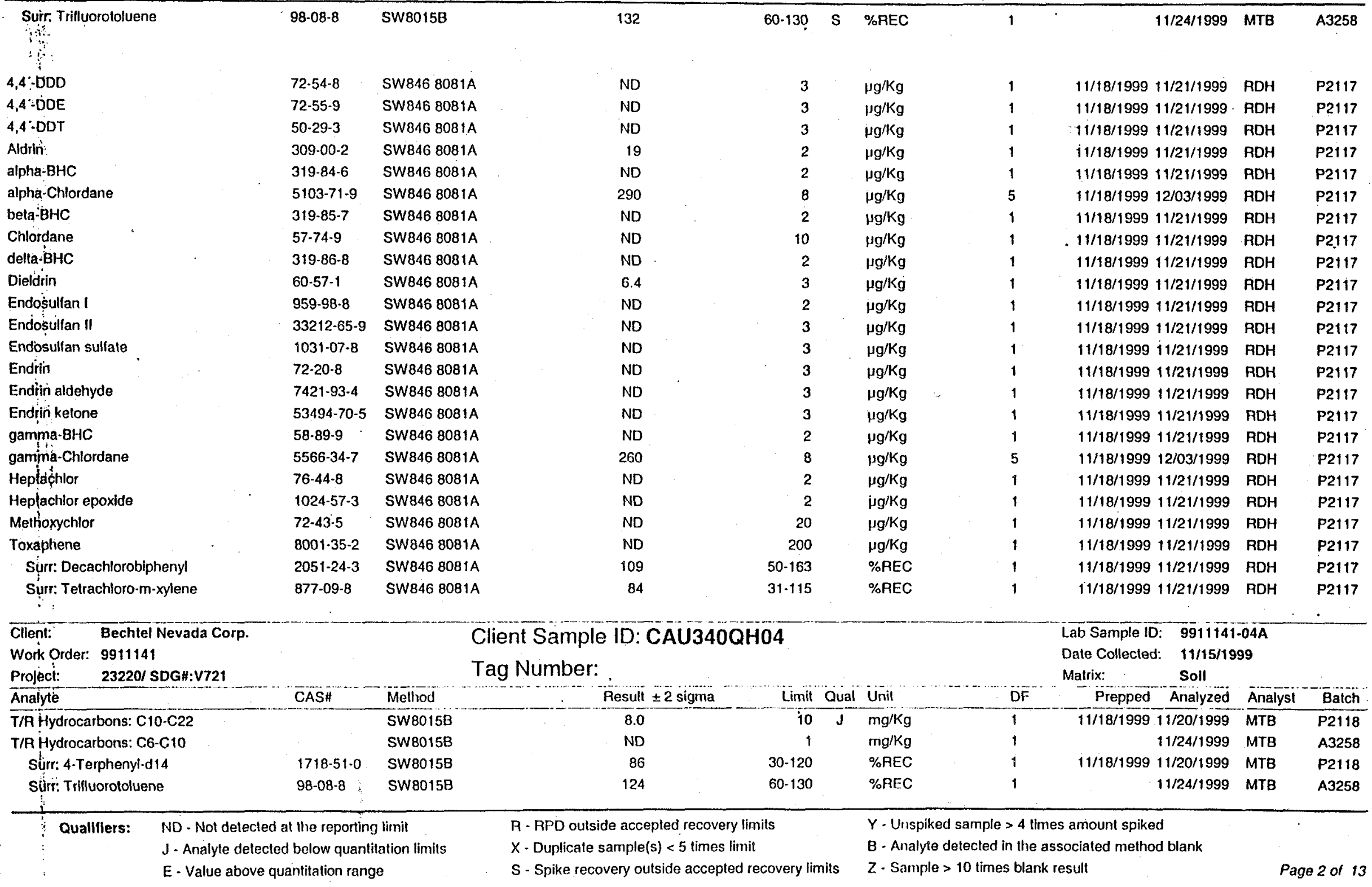




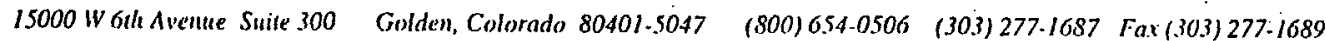

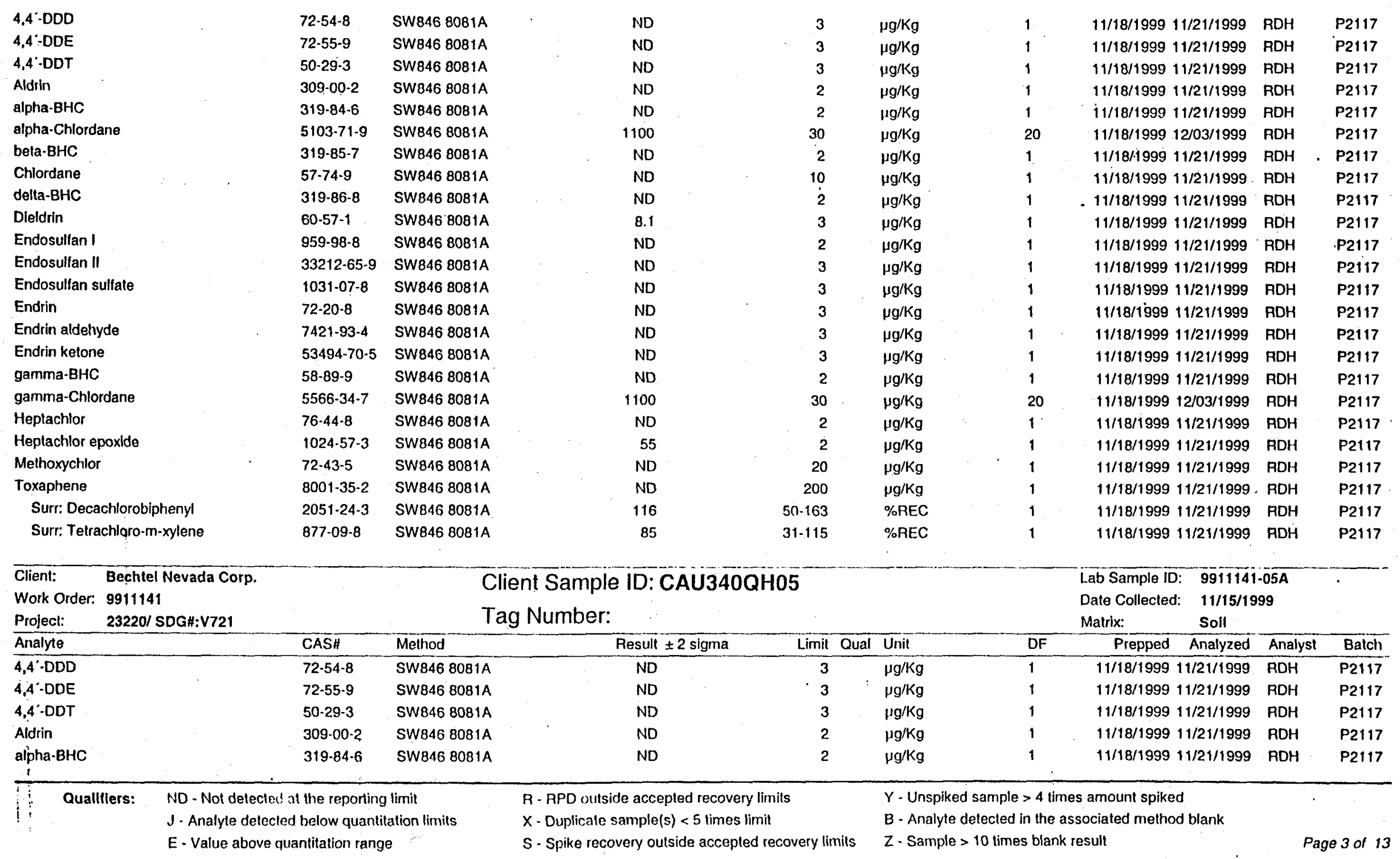


Date: $07-D e c-99$

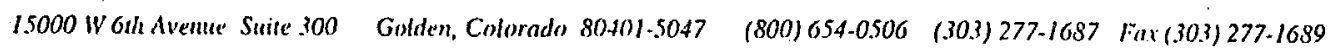

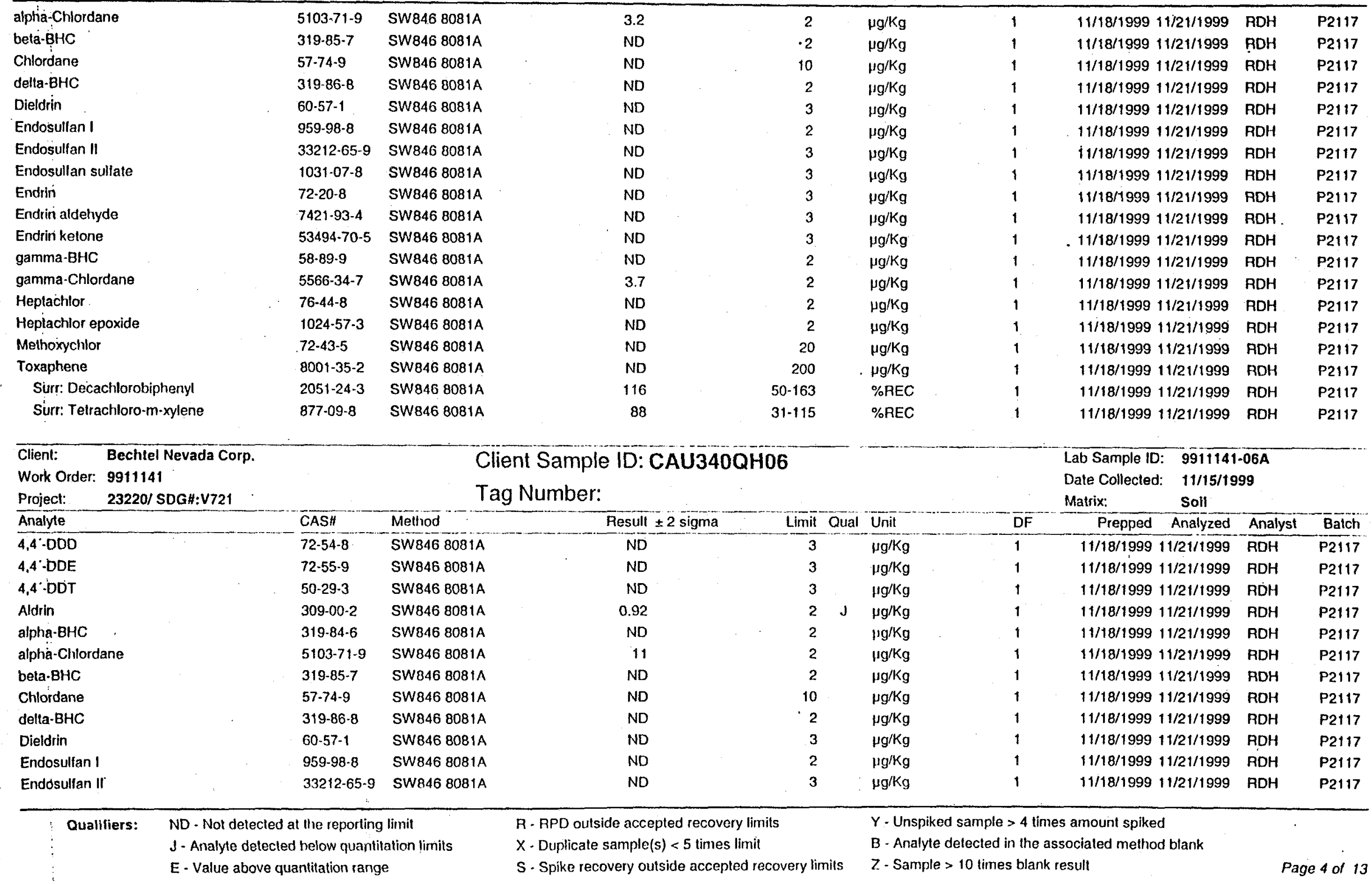

Analyte detecled helow quantitation limits

S. Spike recovery outside accepted recovery limits

$Z$ - Sample $>10$ times blank resull 
Date: 07-Dec-99 Barringer Laboratories, Inc.

CLIENT SAMPLE REPORT

15000 W Gth Avenue Suite 300 Golden, Colorado 80401.5047 (800) 6.54-0.500 (303) 277.1687 Far (303) 277.1689

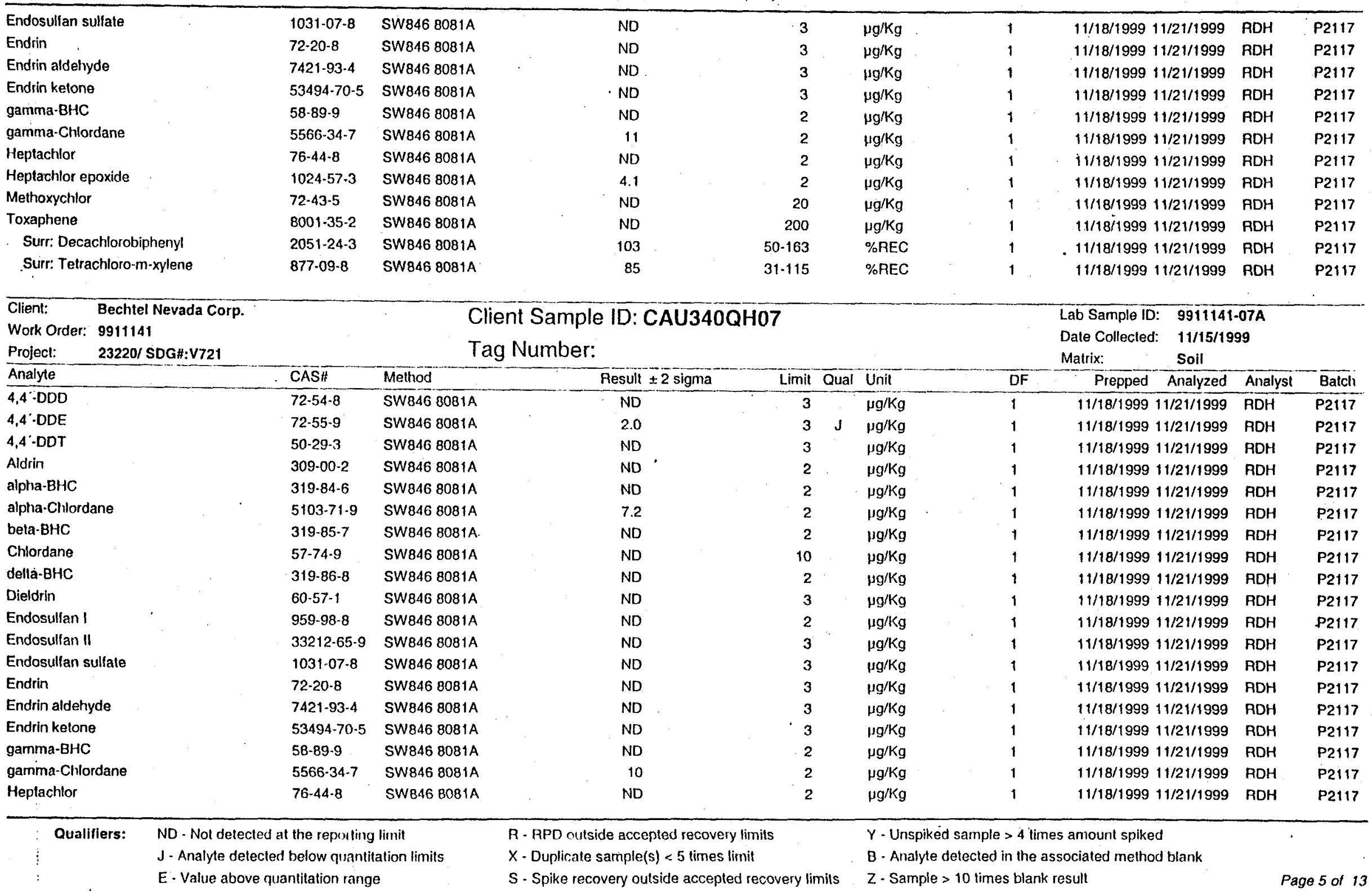


Date: 07-Dec-99 Barringer Laboratories, Inc.

CLIENT SAMPLE REPORT

15000 W 6 h Aveme Suite 300

Golden, Colorado 80401-5047 (800) 654-0506 (303) 277-1687 Frax (303) 277-1689

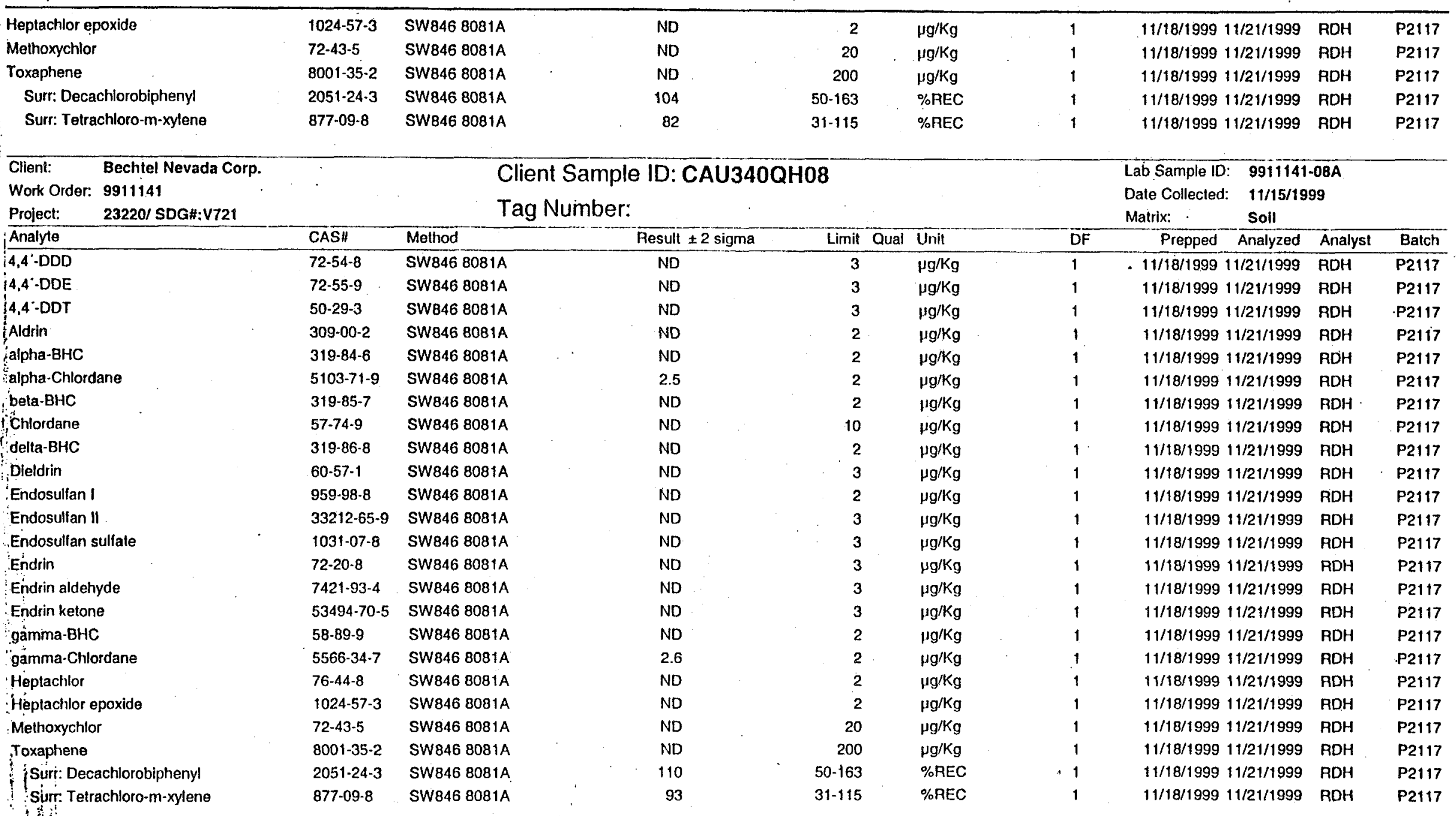
(1)

$\begin{array}{ll}\text { Qualifiers: } & \text { ND - Not detected at the reporting limil } \\ & \text { J - Analyte detected below quantitation limits } \\ & \text { E - Value above quantitation range }\end{array}$


Date: 07-Dec-99 Barringer Laboratories, Inc.

CLIENT SAMPLE REPORT

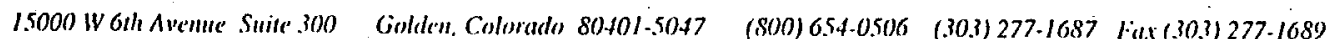

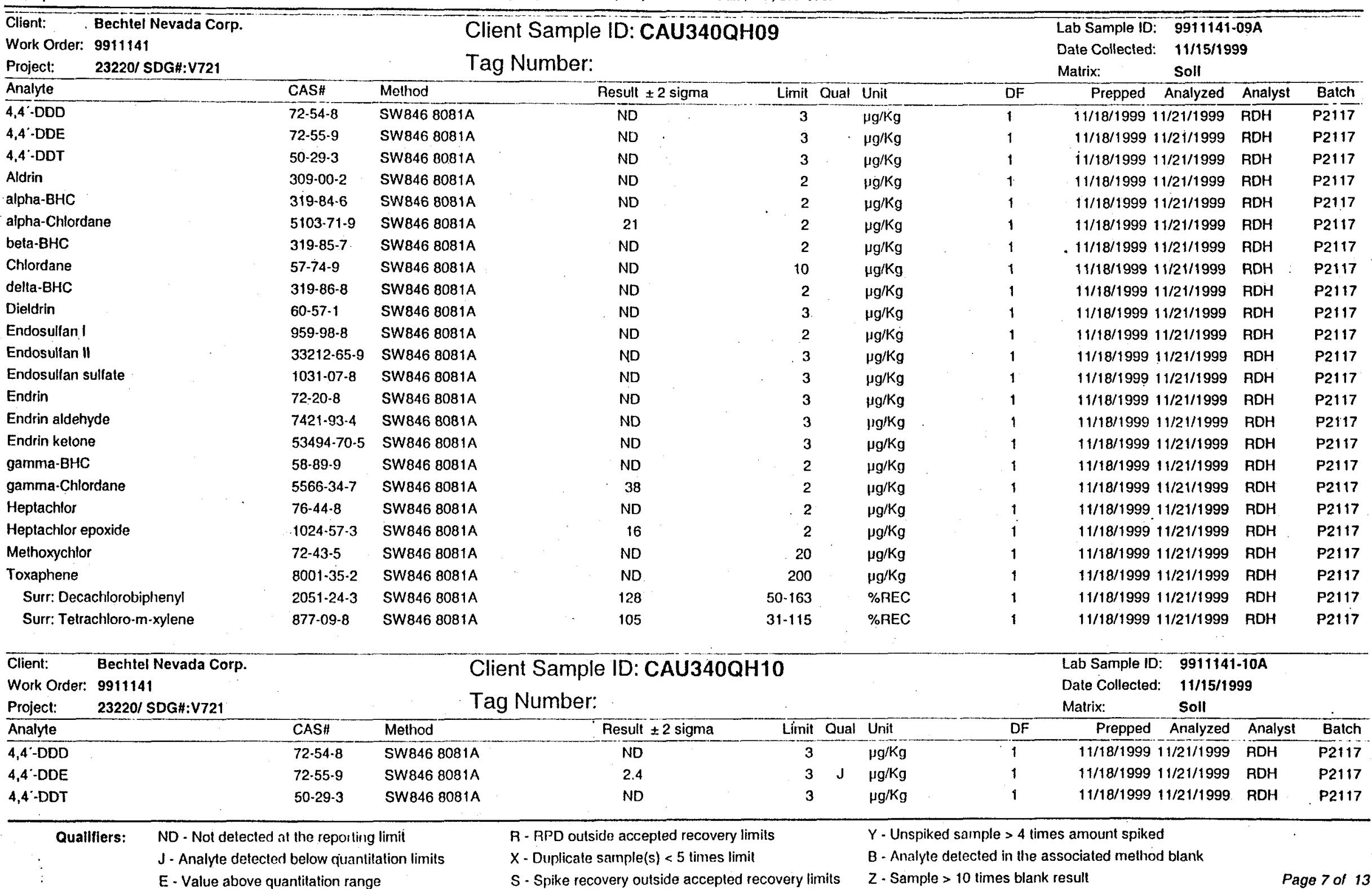


Date: 07-Dec-99 Barringer Laboratories, Inc.

CLIENT SAMPLE REPORT

15000 W Gth Avenue Suire 300 Golden. Colorado 80401-5047 (800)654-0506 (30.3) 277.1687 Fax (303) 277.1689

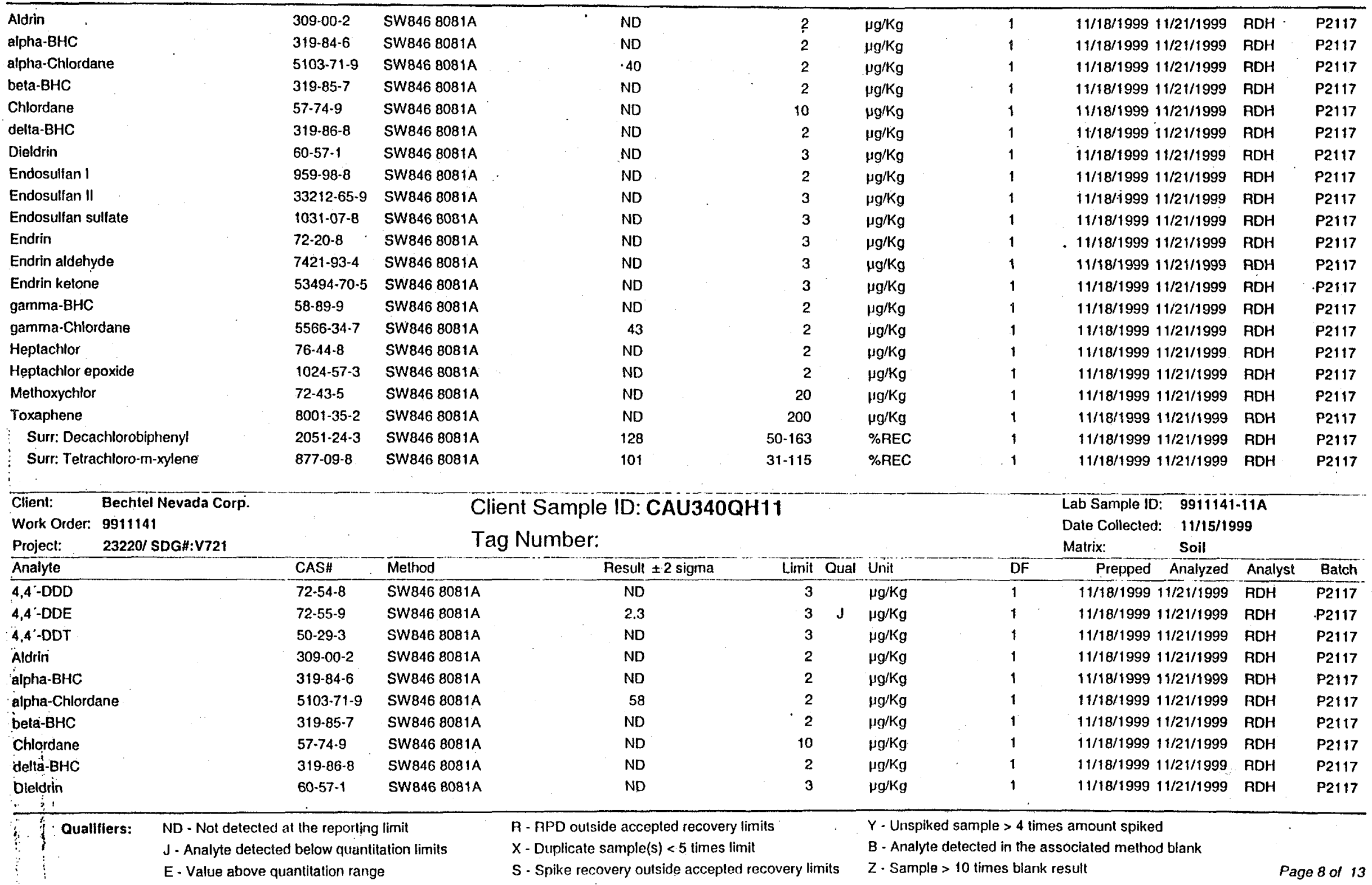


Date: 07-Dec-99

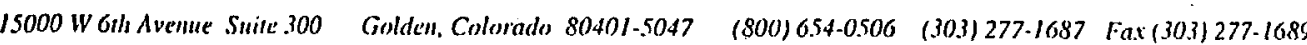

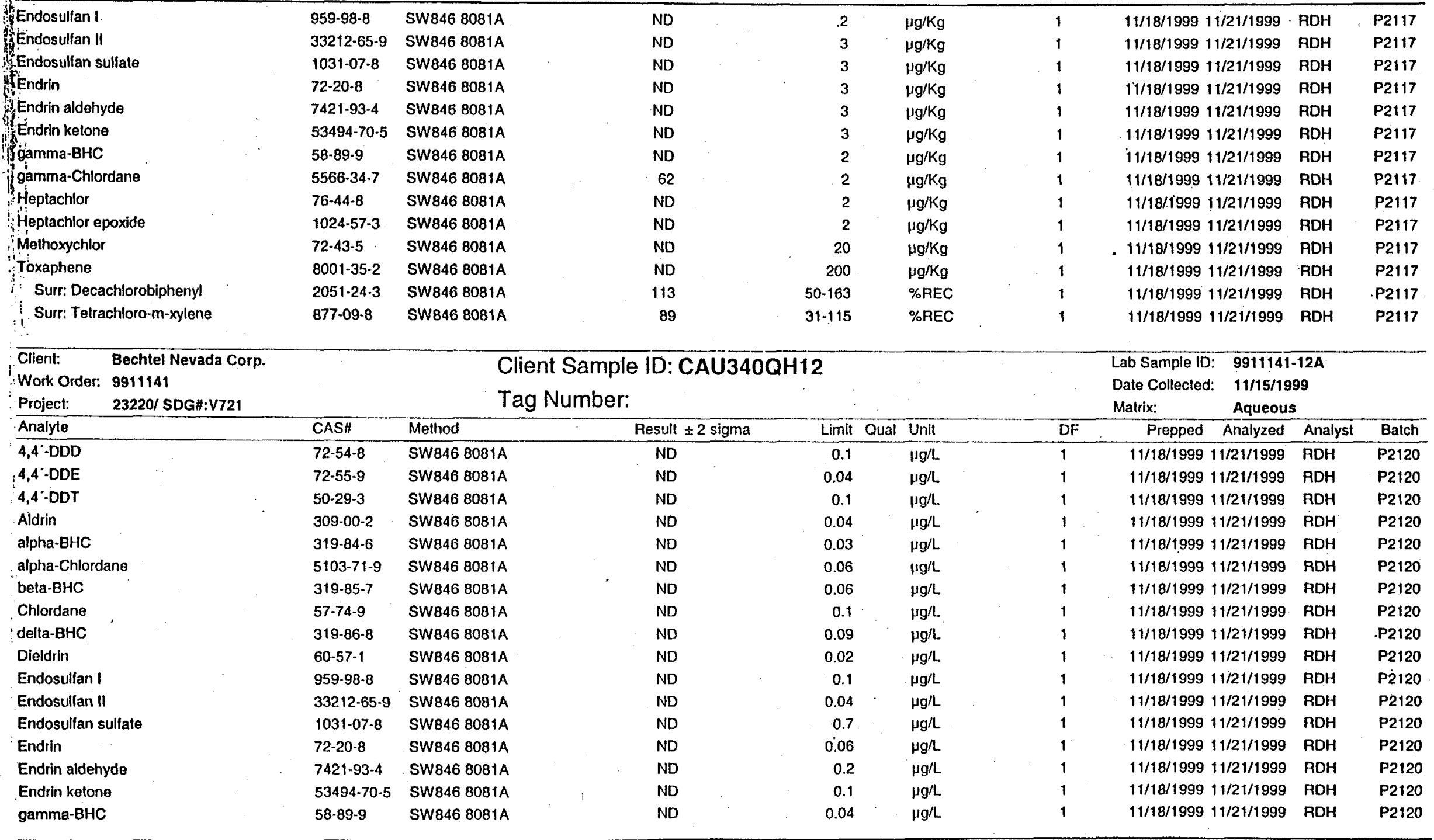

gamma-BHC

R - RPD outside accepted recovery limits

$X$ - Duplicate sample(s) $<5$ times limit

S - Spike recovery outside accepted recovery limits
$Y$ - Unspiked sample $>4$ times amount spiked

$B$ - Analyte detected in the associated method blank

$Z$ - Sample $>10$ times blank result
$J$ - Analyte delected below quantitation limits

$E$ - Value above quantitation range 
I5000 W6Ih Avemue Suite 300 Golden, Colorado 80401-5047 (500)654-0506 (303) 277.1687 Fax (303) 277-1689

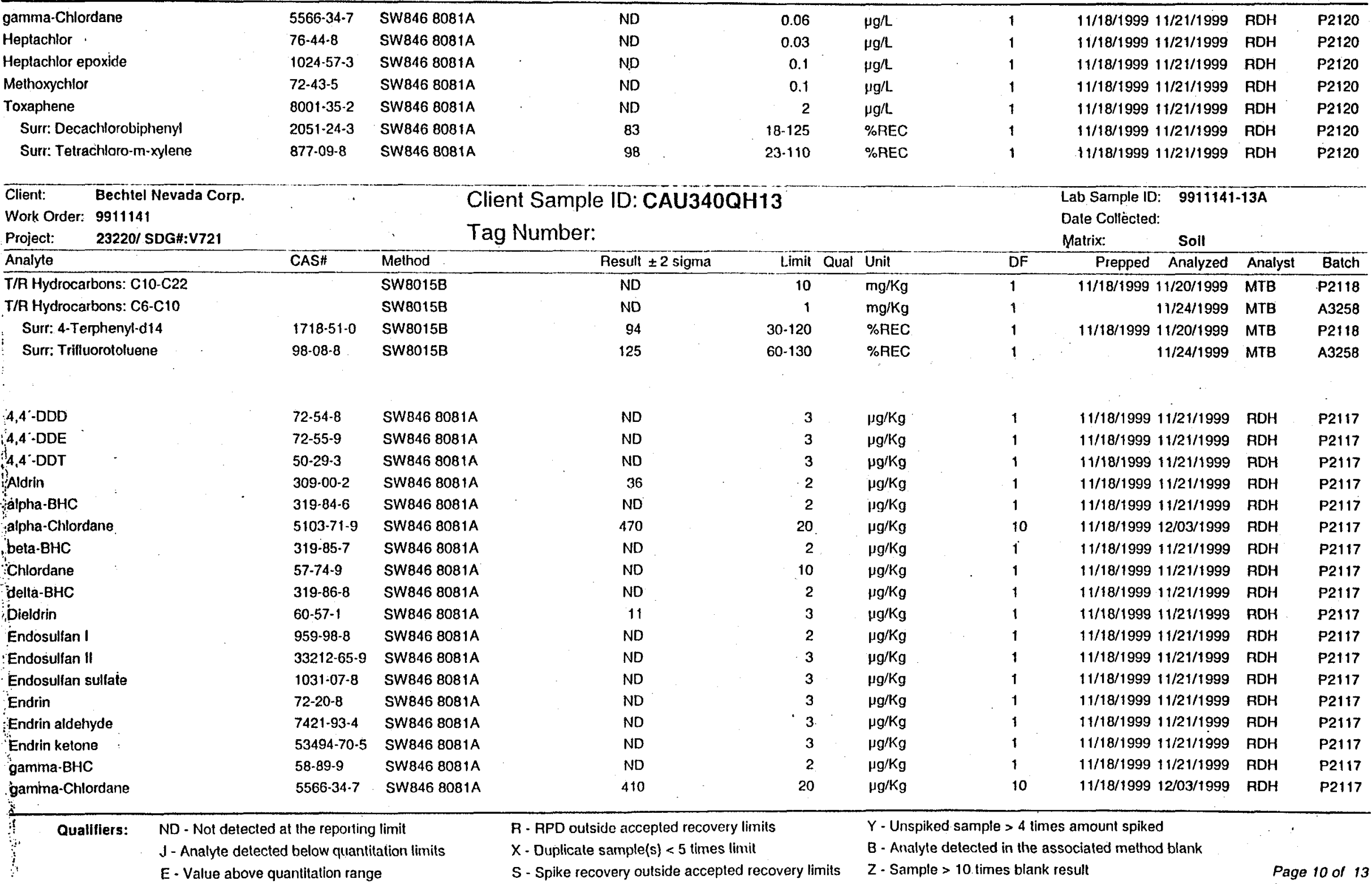

E. Value above quantitation range

$X$ - Duplicale sample(s) $<5$

B - Analyle delecled in the associale 
Date: 07-Dec-99 Barringer Laboratories, Inc.

CLIENT SAMPLE REPORT

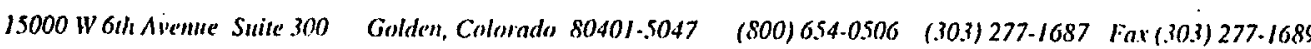

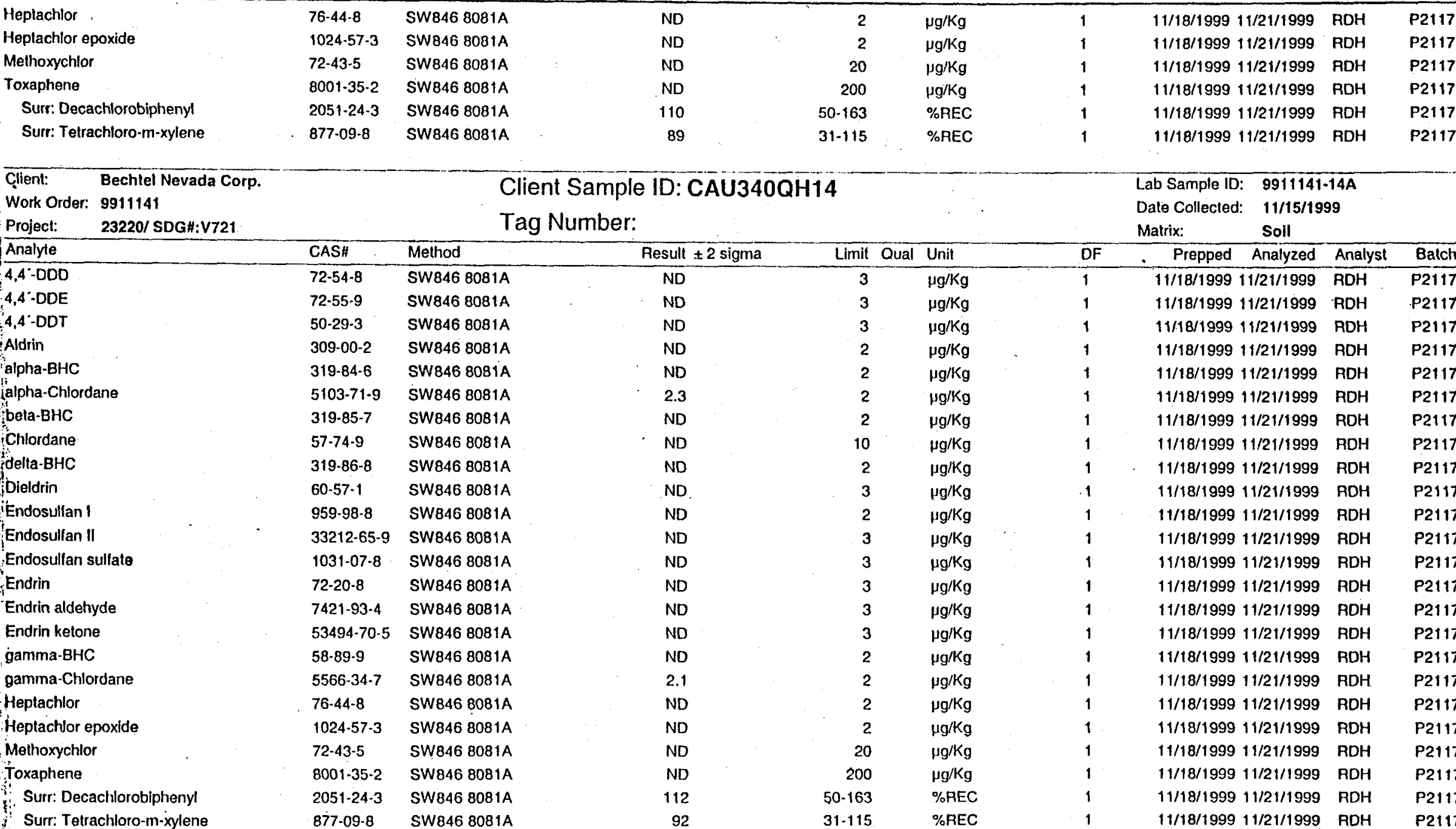

Surr: Tetrachloro-m-xylene

877-09-8 SW846 8081A

$Y$ - Unspiked sample $>4$ times amount spiked

A - RPD outside accepted recovery limits

$B$ - Analyte detected in the associated method blank

$\mathrm{J}$ - Analyte detecled below quantitation limits

$X$ - Duplicate sample $(s)<5$ times limi

$Z \cdot$ Sample $>10$ times blank resul 
Date: $07-$ Dec-99 Barringer Laboratories, Inc.

CLIENT SAMPLE REPORT

15000 W 6th Avenue Suite 300 Golden, Colorado 80401.5047 $\quad$ (800)654-0506 (303) 277.1687 Fax (303) 277-1689

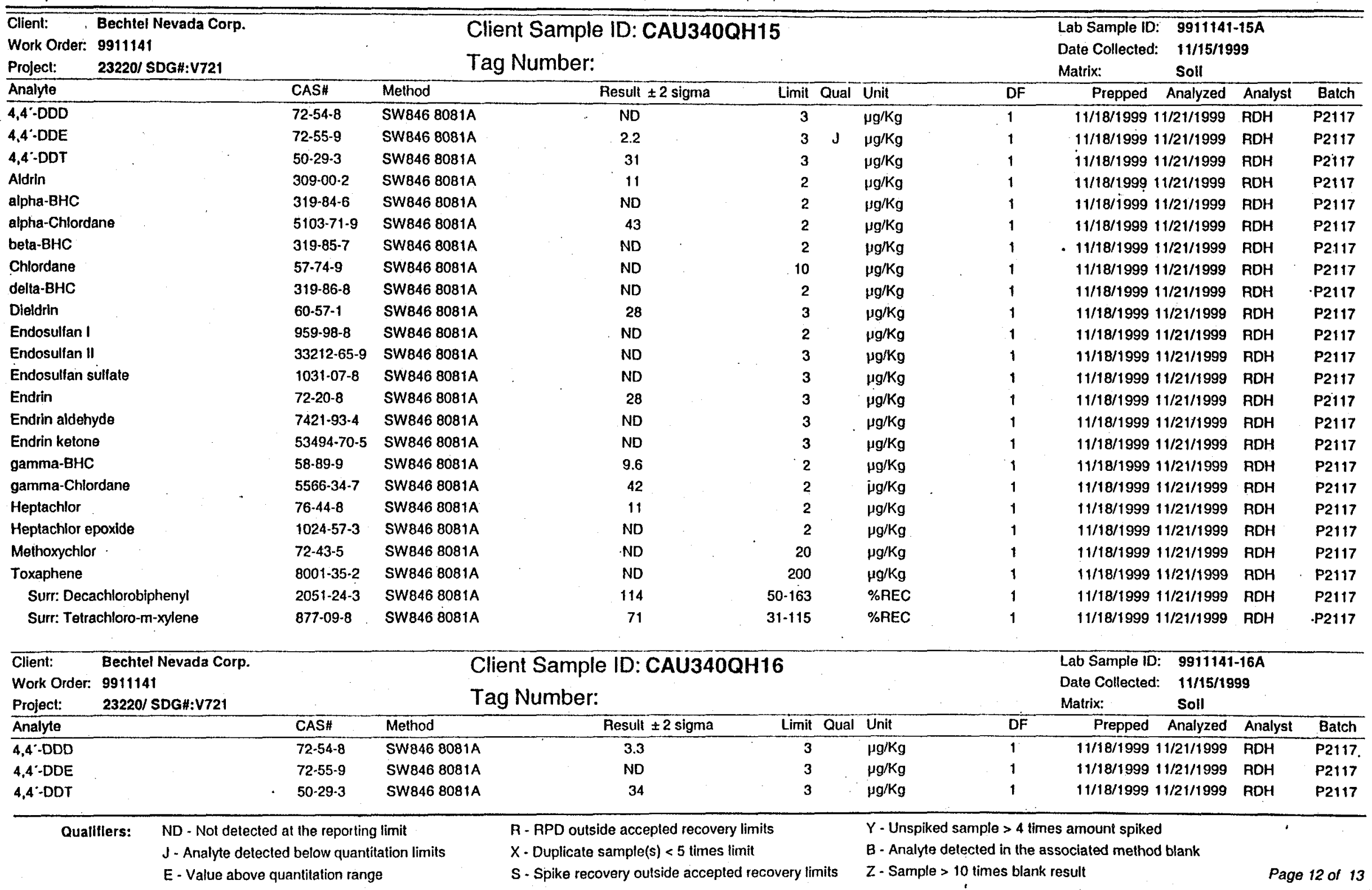


Date: 07-Dec-99

Barringer Laboratories, Inc.

\section{CLIENT SAMPLE REPORT}

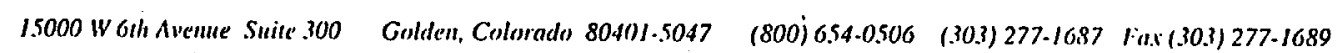

\begin{tabular}{|c|c|c|c|c|c|c|c|c|c|c|}
\hline Aldrin & $309-00-2$ & SW846 8081A & 9.4 & & 2 & $\mu g / \mathrm{Kg}$ & 1 & $11 / 18 / 1999$ 11/21/1999 & RDH & P2117 \\
\hline alpha-BHC. & $319-84-6$ & SW846 $8081 A$ & ND & . & 2 & $\mu g / \mathrm{Kg}$ & 1 & $11 / 18 / 199911 / 21 / 1999$. & $\mathrm{ADH}$ & P2117 \\
\hline alpha-Chlordane & $5103-71-9$ & SW846 8081A & 7.9 & & 2 & $\mu g / \mathrm{Kg}$ & 1 & $11 / 18 / 199911 / 21 / 1999$ & $\mathrm{RDH}$ & P2117 \\
\hline beta-BHC & $319-85-7$ & SW846 $8081 A$ & ND & & 2 & $\mu g / \mathrm{Kg}$ & 1 & $11 / 18 / 1999$ 11/21/1999 & $\mathrm{RDH}$ & P2117 \\
\hline Chlordane & $57-74-9$ & SW846 $8081 \mathrm{~A}$ & ND & & 10 & $\mu \mathrm{g} / \mathrm{Kg}$ & 1 & $11 / 18 / 199911 / 21 / 1999$ & $\mathrm{RDH}$ & P2117 \\
\hline delta-BHC & $319-86-8$ & SW846 8081A & ND & & 2 & $\mu \mathrm{g} / \mathrm{Kg}$ & 1 & $11 / 18 / 1999$ 11/21/1999 & $\mathrm{RDH}$ & P2117 \\
\hline Dieldrin & $60-57-1$ & SW846 8081A & 27 & & 3 & $\mu g / \mathrm{Kg}$ & 1 & $11 / 18 / 1999$ 11/21/1999 & RDH & P2117 \\
\hline Endosulfan I & $959-98-8$ & SW846 8081A & ND & & 2 & $\mu g / \mathrm{Kg}$ & 1 & 11/18/1999 11/21/1999 & $\mathrm{RDH}$ & P2117 \\
\hline Endosulfan II & $33212-65-9$ & SW846 $8081 A$ & ND & & 3 & $\mu \mathrm{g} / \mathrm{Kg}$ & 1 & 11/18/1999 11/21/1999 & $\mathrm{RDH}$ & P2117 \\
\hline Endosulfan sulfate & $1031-07-8$ & SW846 $8081 A$ & ND & & 3 & $\mu g / \mathrm{Kg}$ & 1 & 11/18/î999 11/21/1999 & RDH & P2117 \\
\hline Endrin & $72-20-8$ & SW846 8081A & 28 & . & 3 & $\mu g / \mathrm{Kg}$ & 1 & 11/18/1999 11/21/1999 & $\mathrm{RDH}$ & P2117 \\
\hline Endrin aldehyde & $7421-93-4$ & SW846 8081A & ND & $\therefore$ & 3 & $\mu g / \mathrm{Kg}$ & 1 & 11/18/1999 11/21/1999 & $\mathrm{RDH}$ & P2117 \\
\hline Endrin ketone & $53494-70-5$ & SW846 8081A & ND & & 3 & $\mu \mathrm{g} / \mathrm{Kg}$ & 1 & $11 / 18 / 1999 \quad 11 / 21 / 1999$ & $\mathrm{RDH}$ & $P 2117$ \\
\hline gamma-BHC & $58-89.9$ & SW846 8081A & 9.1 & & 2 & $\mu g / K g$ & 1 & $11 / 18 / 1999$ 11/21/1999 & $\mathrm{RDH}$ & P2117 \\
\hline gamma-Chlordane & $5566-34-7$ & SW846 $8081 A$ & 12 & & 2 & $\mu g / \mathrm{Kg}$ & 1 & $11 / 18 / 199911 / 21 / 1999$ & $\mathrm{RDH}$ & P2117 \\
\hline Heplachlor & $76-44-8$ & SW846 8081A & 10 & & 2. & $\mu \mathrm{g} / \mathrm{Kg}$ & 1 & $11 / 18 / 199911 / 21 / 1999$ & $\mathrm{RDH}$ & P2117 \\
\hline Heptachlor epoxide & $1024-57-3$ & SW846 8081A & 3.8 & & 2 & $\mu g / K g$ & 1 & $11 / 18 / 199911 / 21 / 1999$ & $\mathrm{RDH}$ & P2117 \\
\hline Methoxychlor & $72-43-5$ & SW846 8081A & ND & & 20 & $\mu g / \mathrm{Kg}$ & 1 & $11 / 18 / 199911 / 21 / 1999$ & RDH & P2117 \\
\hline Toxaphene & $8001-35-2$ & SW846 8081A & ND & & 200 & $\mu g / \mathrm{Kg}$ & 1 & $11 / 18 / 1999$ 11/21/1999 & $\mathrm{RDH}$ & P2117 \\
\hline Surr: Decachlorobiphenyl & $2051-24-3$ & SW846 8081A & 115 & & $50-163$ & $\%$ REC & 1 & 11/18/1999 11/21/1999 & $\mathrm{RDH}$ & P2117 \\
\hline Surr: Tetrachloro-m-xylene & $877-09-8$ & SW846 8081A & 73 & & $31-115$ & $\%$ REC & 1 & 11/18/1999 11/21/1999 & RDH & P2117 \\
\hline
\end{tabular}

$\begin{array}{ll}\text { Qualliters: } & \text { ND - Not detected at the reporting limit } \\ & J \text { - Analyte detected below quantitation limits } \\ & E-\text { Value above quantitation range }\end{array}$
R - RPD outside accepted recovery limits

$X$ - Duplicale sample $(s)<5$ times limit

S - Spike recovery outside accepted recovery limits
$Y$. Unspiked sample $>4$ limes amount spiked

$B$ - Analyte detected in the associated method blank

$Z$ - Sample $>10$ times blank result 

Date: $07-\operatorname{Dec} \cdot 99$

Barringer Laboratories, Inc.

BATCH QC SUMMARY REPORT

15000 W Gih Avenue Suite 300 Golden, Colorado 80401.5047 (800)654.0506 (30.3) 277.1687 fax (303) 277.1689

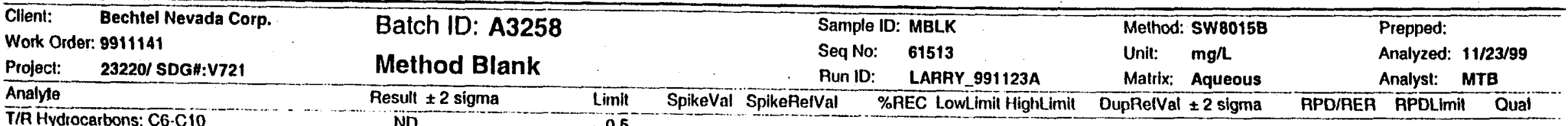

T/R Hydrocarbons: $\bar{C} \cdot \bar{C} \cdot 10$

ND

$0 . \overline{5}$

$135 \quad 60 \quad 130$

$\mathbf{s}$

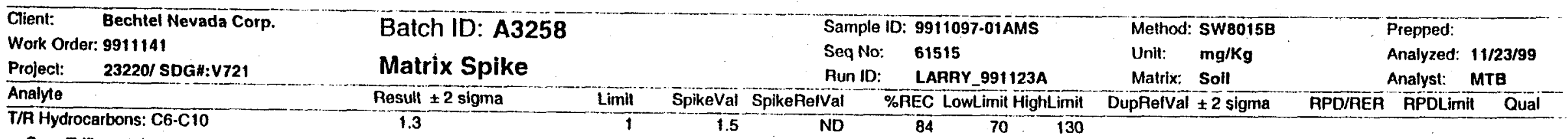

$103 \quad 60 \quad 130$

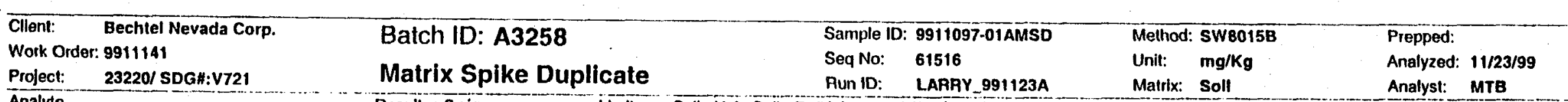

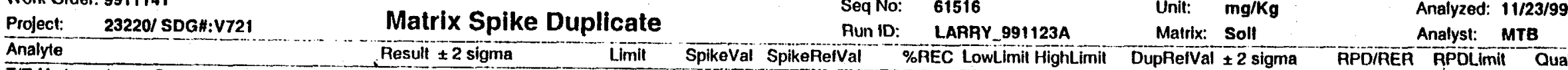

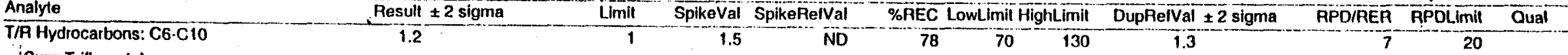

Surr: Trilluorotoluene

$104 \quad 60 \quad 130$

\begin{tabular}{|c|c|c|c|c|c|c|c|c|c|c|c|c|}
\hline \multirow[t]{2}{*}{$\begin{array}{l}\text { Client: } \\
\text { Wechtel Nevada Corp. } \\
\text { Work Order: } 9911141 \\
\text { Project: } \quad 23220 / \text { SDGH:V721 } \\
\text { Anglute }\end{array}$} & \multicolumn{3}{|c|}{$\begin{array}{l}\text { Batch ID: P2117 } \\
\text { Laboratory Control Spike }\end{array}$} & \multicolumn{4}{|c|}{$\begin{array}{ll}\text { Sample ID: } & \text { CCS-2117 } \\
\text { Seq No: } & 60927 \\
\text { Run ID: } & \text { MOE_991120B }\end{array}$} & \multirow{2}{*}{$\begin{array}{l}\text { Method: } \\
\text { Unit: } \\
\text { Matrix: } \\
\text { DupRefVal }\end{array}$} & \multirow{2}{*}{$\begin{array}{l}\text { SW846 Bo } \\
\mu \mathrm{g} / \mathrm{Kg} \\
\text { Soll } \\
2 \text { sigma } \\
\end{array}$} & \multirow{2}{*}{ RPDIRER } & \multicolumn{2}{|c|}{$\begin{array}{l}\text { Prepped: } 11 / 18 / 99 \\
\text { Analyzed: } 11 / 21 / 99 \\
\text { Analyst: } \quad \text { RDH }\end{array}$} \\
\hline & Result \pm 2 sigma & Limit & SpikeVal & SpikeRelval & $\%$ \%EC & LowLimil & timit & & & & APDLimit & Qual \\
\hline $4,4^{\circ} \cdot \mathrm{DDT}$ & 9.7 & 3 & 33 & & 29 & 25 & 160 & & & & & \\
\hline Aidrin & 7.0 & 2 & 13 & & 52 & 42 & 122 & & & & & \\
\hline Dleldrin & 19 & 3 & 33 & & 58 & 36 & 146 & & & & & . \\
\hline Enidrin & 16 & 3 & 33 & & 49 & 30 & 147 & & & & & \\
\hline gămma-BHC & 7.1 & 2 & 13 & & 54 & 32 & 127 & & & & & \\
\hline Heptachlor & 5.1 & 2 & 13 & & 38 & 34 & 111 & & . & & & \\
\hline Surr: Decachlorobiphenyl & & & & & 78 & 50 & 163 & & & & & \\
\hline Surr: Tetrachloro-m-xylene & & & & & 72 & 31 & 115 & & & & & \\
\hline
\end{tabular}

\begin{tabular}{|c|c|c|c|}
\hline Quall & $\begin{array}{l}\text { ND - Nol detected at the reporting limit } \\
J \text { - Analyte delected below quantilation limils } \\
\text { E - Value above quantilation range }\end{array}$ & $\begin{array}{l}\text { R- RPD outside accepled recovery limits } \\
X \text { - Duplicale sample(s) < } 5 \text { times limil } \\
\text { S- Spike recovery outside accepted recovery limits }\end{array}$ & $\begin{array}{l}Y \text { - Unspiked sample }>4 \text { times amount spiked } \\
\text { B. Analyte delected in the associated method blank } \\
Z \text {. Sample }>10 \text { times blank result }\end{array}$ \\
\hline
\end{tabular}

Page 1 of 6 
Date: $07-$ Dec-99 Barringer Laboratories, Inc.

I5000 W 6th Avenue Suite 300 Gulden, Colorado 80401.5047 (800) 654.0506 (303) 277.1687 Far (303) 277-1689

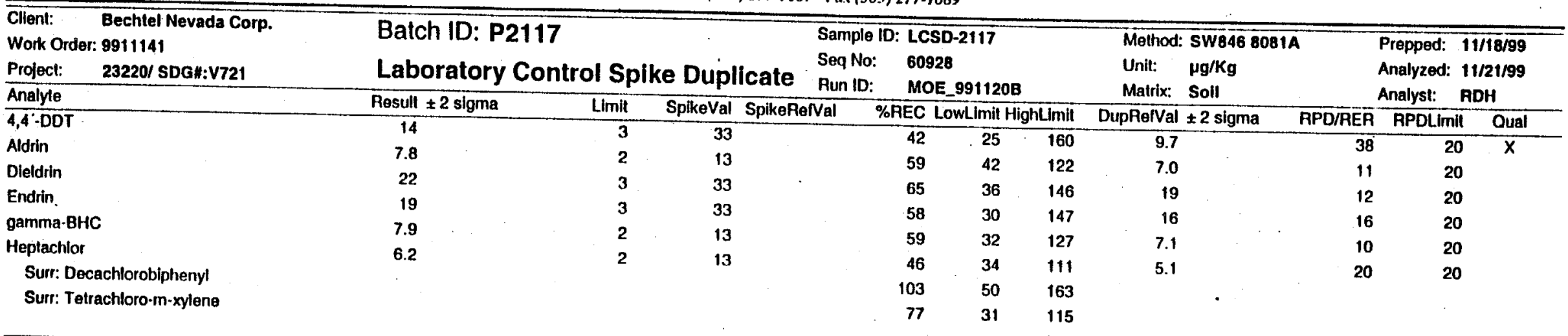

\begin{tabular}{|c|c|c|c|c|c|c|c|c|c|c|c|c|}
\hline \multirow{2}{*}{\multicolumn{2}{|c|}{$\begin{array}{l}\text { Client: } \\
\text { Work Order: } 9911141 \\
\text { Project: } 23220 / \text { SDGH:V721 } \\
\text { Analyte }\end{array}$}} & \multicolumn{2}{|c|}{$\begin{array}{l}\text { Batch ID: P2117 } \\
\text { Method Blank }\end{array}$} & & & \multicolumn{2}{|c|}{$\begin{array}{ll}\text { Sample ID: } & \text { MBLK-2117 } \\
\text { Seq No: } & 60926 \\
\text { Fun ID: } & \text { MOE_991120B } \\
\end{array}$} & \multicolumn{2}{|c|}{$\begin{array}{l}\text { Method: } 5 W 8468081 A \\
\text { Unit: }\end{array}$} & & \multicolumn{2}{|c|}{$\begin{array}{ll}\text { Prepped: } & 11 / 18 / 99 \\
\text { Analyzed: } & 11 / 21 / 99 \\
\text { Analyst: } & \text { RDH } \\
\end{array}$} \\
\hline & & Resull \pm 2 sigma & Limit & SplkeVal & SpikeRelval & $\%$ AEC LOY & owLimit HighLimil & DupRelVal & \pm 2 sigma & APD/AER & APDLimit & Qual \\
\hline $4,4^{\circ} \cdot \mathrm{DDD}$ & & ND & 3 & & & & & & & & & \\
\hline $\begin{array}{l}4,4^{\circ}-\mathrm{DDE} \\
4,4^{\circ}-\mathrm{DDT}\end{array}$ & & ND & 3 & & & & & & & & & \\
\hline $\begin{array}{l}4,4^{\circ}-\mathrm{DDT} \\
\text { Aldrin }\end{array}$ & & ND & 3 & & & & & . & & & & \\
\hline $\begin{array}{l}\text { Aldrin } \\
\text { alpha-BHC }\end{array}$ & & No & 2 & & & & & & & & & \\
\hline $\begin{array}{l}\text { alpha-BHC } \\
\text { alpha-Chlordane }\end{array}$ & & ND & 2 & & & & & & & & & \\
\hline $\begin{array}{l}\text { alpha-Chlordane } \\
\text { beta-BHC }\end{array}$ & & ND & 2 & & & & & & & & & \\
\hline $\begin{array}{l}\text { beta-BHC } \\
\text { Chlordane }\end{array}$ & & ND & 2 & & & & & . & & & & \\
\hline $\begin{array}{l}\text { Chlordane } \\
\text { della-BHC }\end{array}$ & . & ND & 10 & & & & & & & & & \\
\hline $\begin{array}{l}\text { della-BHC } \\
\text { Dieldrin }\end{array}$ & & ND & $\begin{array}{l}2 \\
3\end{array}$ & & & & & & & & & \\
\hline $\begin{array}{l}\text { Dieldrin } \\
\text { Endosulfan I }\end{array}$ & & ND & $\begin{array}{l}3 \\
2\end{array}$ & &. & & & & & & & \\
\hline $\begin{array}{l}\text { Endosullan I } \\
\text { Endosullan II }\end{array}$ & & ND & $\begin{array}{l}2 \\
3\end{array}$ & & & & & & & & & · \\
\hline $\begin{array}{l}\text { Endosulfan II } \\
\text { Endosulfan sulfate }\end{array}$ & & ND & $\begin{array}{l}3 \\
\mathbf{3}\end{array}$ & & & & & . & & & & \\
\hline $\begin{array}{l}\text { Endosulfan sulfate } \\
\text { Endrin }\end{array}$ & & ND & $\begin{array}{l}3 \\
3\end{array}$ & & & & & & & & & \\
\hline $\begin{array}{l}\text { Endrin } \\
\text { Endrin aldehyde }\end{array}$ & & $\begin{array}{l}\text { ND } \\
\text { ND }\end{array}$ & 3 & & & & & & & & & \\
\hline Endrin ketone & & ND & 3 & & & & & & & & & \\
\hline gamma-BHC & & ND & 2 & & & & & & & & & \\
\hline gamma-Chlordan $B$ & & ND & 2 & & & & & & & & & \\
\hline Heplachlor & & ND & 2 & & & & & & & & & \\
\hline Qualifiers: & $\begin{array}{l}\text { ND - Nol detect } \\
\text { J - Analyte dele } \\
\text { E - Value above }\end{array}$ & $\begin{array}{l}\text { reporting limit } \\
\text { ow quantilation limits } \\
\text { ation range }\end{array}$ & $\begin{array}{l}\text { R - RPD } \\
\text { X-Dupli } \\
\text { S-Spike }\end{array}$ & $\begin{array}{l}\text { utside accep } \\
\text { te sample(s } \\
\text { ecovery outs }\end{array}$ & $\begin{array}{l}\text { pted recovery lir } \\
\text { s) }<5 \text { times limi } \\
\text { side accepted }\end{array}$ & covery limils & $\begin{array}{l}Y \text { - Unspiked } \\
\text { B-Analyte det } \\
Z \text { - Sample }>1\end{array}$ & $\begin{array}{l}\text { sample }>4 \text { tim } \\
\text { tecled in the a } \\
10 \text { times blank }\end{array}$ & $\begin{array}{l}\text { mes amount } \\
\text { associated } \mathrm{m} \\
\text { k result }\end{array}$ & $\begin{array}{l}\text { spiked } \\
\text { nelhod blank }\end{array}$ & & Page 2 \\
\hline
\end{tabular}


Date: $07-$ Dec-99 Barringer Laboratories, Inc.

BATCH QC SUMMARY REPORT

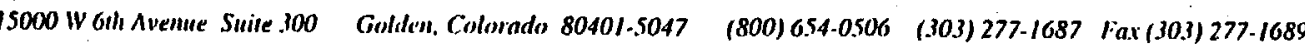

$\begin{array}{llr}\text { Heptachlor epoxide } & \text { ND } & 2 \\ \text { Melhoxychlor } & \text { ND } & 20 \\ \text { Toxaphene } & \text { ND } & 200\end{array}$

Surr: Decachtorobiphenyl

Surr: Tetrachloro-m-xylene

200

\section{Client: Bechtel Nevada Corp.}

Work Order: 9911141

Project: 23220/ SDG: :V721

Batch ID: P2117

Matrix Spike

Seq No: 60942

Result \pm 2 sigma

Run ID: MOE_991120B

(U)

4.4.DDT

$4.4 \cdot \mathrm{DD}$

Aldrin

Dieldrin

Endrin

gamma-BHC

11

Ral \%REC

SpikeVal SpikeReIV

REC LowLimit Highlimil DupRefVal \pm 2 sigma RPD/RER RPOLlimil Qual

Heplachior

28

28

9.6

Surr: Decachlorobiphenyl

Surr: Telrachloro-m-xylen

Client: Bechtel Nevada Corp.

Work Order: 9911141

Project: 2322p/ SDG\#:V721

Batch ID: P2117

$\begin{array}{ll}33 & \mathrm{~N} \\ 13 & 0.9\end{array}$

$\begin{array}{rrrr}0.92 & 77 & 10 & 142\end{array}$

33 ND

ND

ND

83

85

$72 \quad 30$

$82 \quad 18 \quad 141$

$11450 \quad 163$

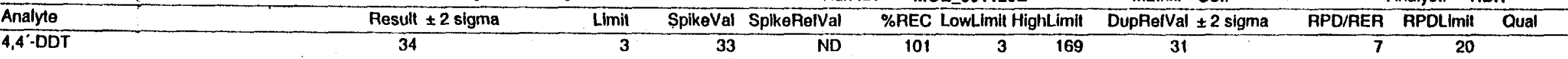

Dieldrin

Endrin

gamma-BHC

Heplachlor

27

28

9.1

Surr: Decachlorobiphenyl

10

Matrix Spike Duplicate

Sample 1D: 9911141-06AMSD

115

Surr: Telrachloro-m-xylene

\begin{tabular}{|c|c|c|c|c|c|c|c|c|c|c|c|}
\hline $\begin{array}{l}\text { Client: } \\
\text { Work O } \\
\text { Project: }\end{array}$ & $\begin{array}{l}\text { Bechlel Nevada Corp. } \\
9911141 \\
23220 / \text { SDG"l:V721 }\end{array}$ & \multicolumn{3}{|c|}{$\begin{array}{l}\text { Batch ID: P2118 } \\
\text { Laboratory Control Spike }\end{array}$} & $\begin{array}{l}\text { San } \\
\text { Seq } \\
\text { Pur }\end{array}$ & $\begin{array}{l}\text { 10: } \operatorname{LCS}-2118 \\
61415 \\
\quad \text { CURLY_991120A }\end{array}$ & $\begin{array}{l}\text { Method: } \\
\text { Unit: } \\
\text { Matrix: }\end{array}$ & $\begin{array}{l}\text { SWBo15B } \\
\mathrm{mg} / \mathrm{Kg} \\
\text { Soll }\end{array}$ & \multicolumn{3}{|c|}{$\begin{array}{ll}\text { Prepped: } & 11 / 18 / 99 \\
\text { Analyzed: } & 11 / 20 / 99 \\
\text { Analyst: } & \text { MTB }\end{array}$} \\
\hline Analyto & & Resyll \pm 2 sigma & Limit & SpikeVal & SpikeReival & \%REC LowLimit HighLimit & DupReival & \pm 2 sigma & RPD/RER & APDLimit & Oual \\
\hline
\end{tabular}

Quallilers: ND - Not detected at the reporting limit

$J$ - Analyte delected below quantilation limits

E - Value above quantitation range
R - APD outside accepted recovery limils

$\dot{X}$ - Duplicate sample(s) $<5$ times limil

S - Spike recovery outside accepled recovery limits

$Y$ - Unspiked sample $>4$ times amount spiked

$B$ - Analyte delecled in the associaled method blank

$Z$. Sample $>10$ limes blank result

Page 3 of 6 
Date: 07-Dec-9G Barringer Laboratories, Inc.

BATCH QC SUMMARY REPORT

15000 W Gth Avemue Suite 300 Golden, Colorado 80401.5047 (800) 654-0506 (30.3) 277.1687 Far (303) 277.1689

T/R Hydrocarbons: C10-C22

17

10

33

Surr: 4-Terphenyl-d14

$52 \quad 30 \quad 120$

Client: . Bechtel Nevada Corp.

Work Order: 9911141

Project: · 23220/ SDG\#:V721

Batch ID: P2118

91

120

T/F Hydrocarbons: C10-C22

Surr: 4-Terphenyl-d 14

Laboratory Control Spike Duplicate

Sample 1D: LCSD-2118

Seq No: 61416

Run ID: CURLY_991120A

Mellod: SWB0158

Prepped: 11/18/99

Result \pm 2 sigma L__ Limil

33

\%AEC Low Imit MighLimit

Analyzed: 11/20/99

Matrix: Soll

Analyst: MTB

$30 \quad 120$

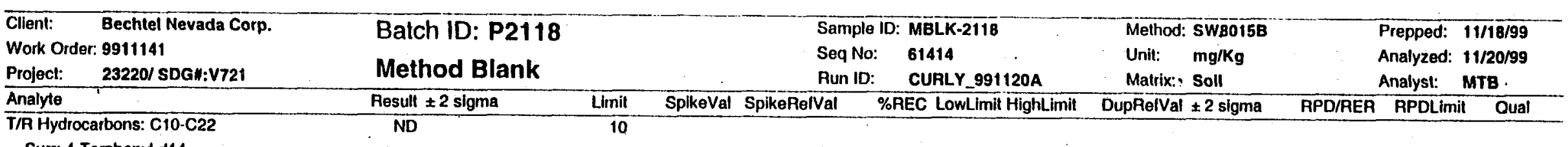

Surr: 4-Terphenyl-d14

$92 \quad 30 \quad 120$

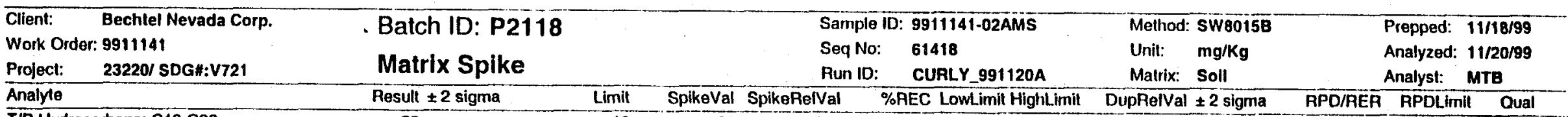

T/R Hydrocarbons: C10-C22

Hesull \pm 2 sigma L Limil SpikeVal SpikeRelVal \%AEC LowLimil HighLimit

DupReival \pm 2 sigma

Surr: 4-Terphenyl-d 14

$90 \quad 30 \quad 120$

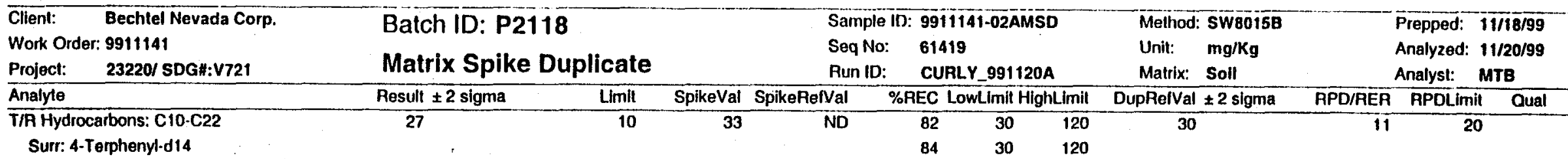

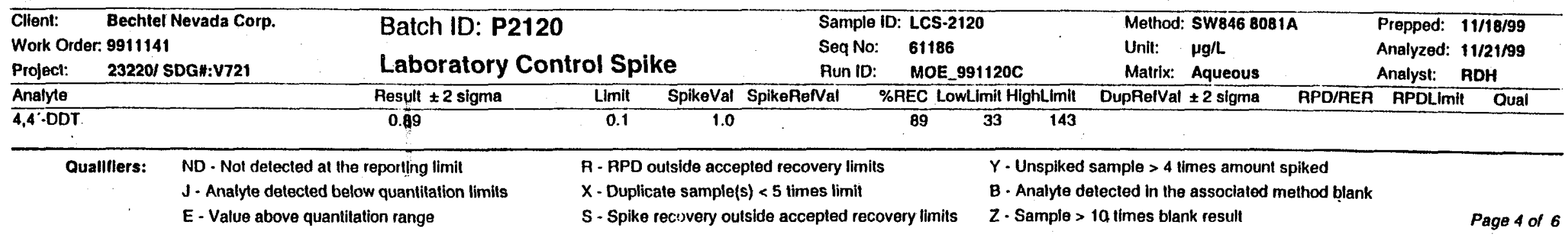


\&
$\dot{d}$
$\stackrel{0}{0}$
பे
$\ddot{0}$
$\frac{0}{0}$

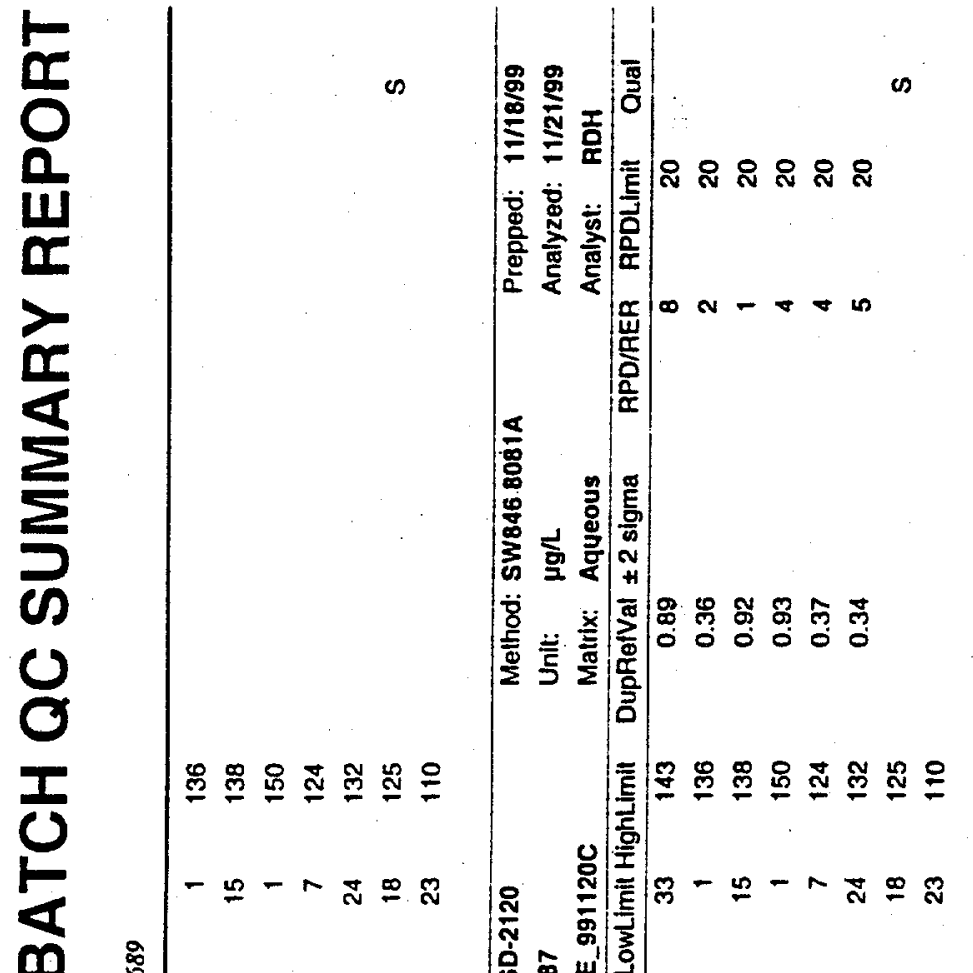

๓

웅 으 우 융 웅

:

ปั่

๑ัญ

훙 융 융

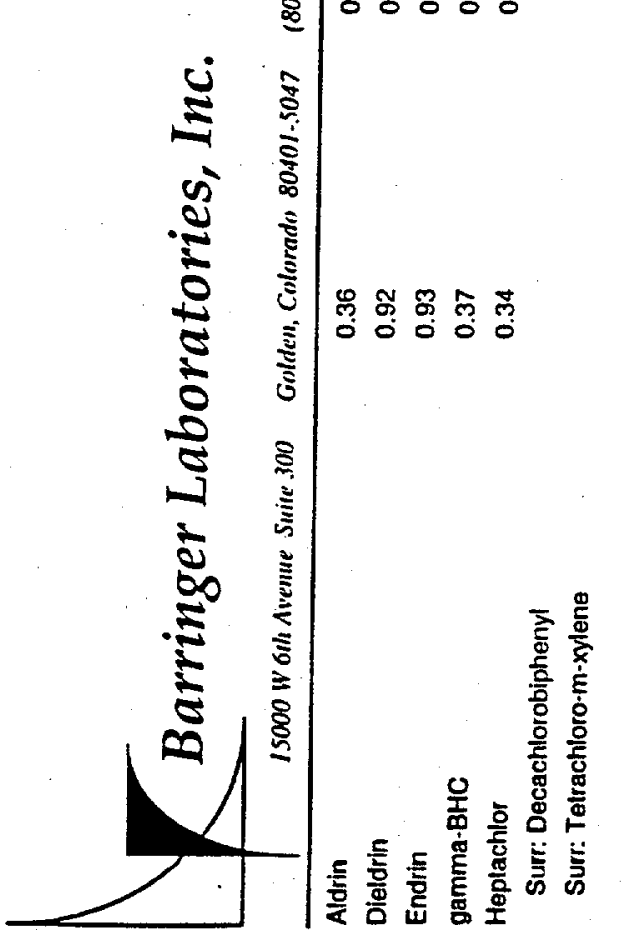

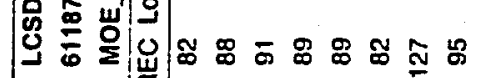

空

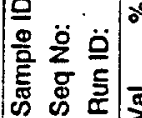

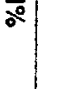

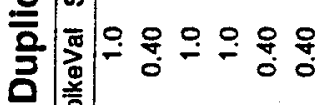

$\underline{\underline{x}}$

官

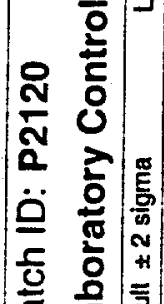

吾

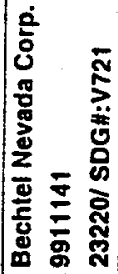

놌 행ㅎㅀ

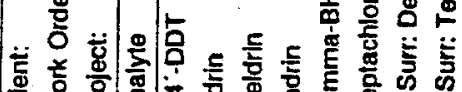

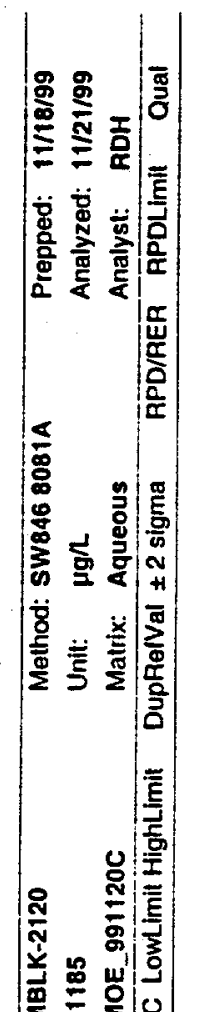

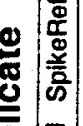

E्ञ

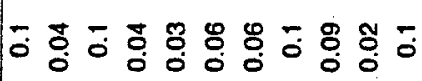

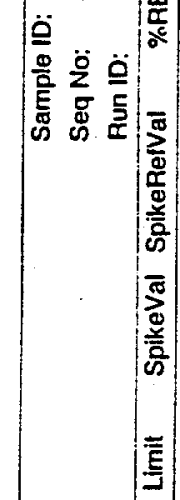

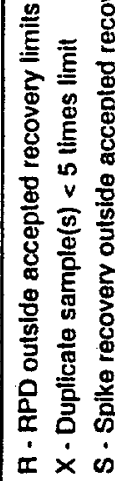

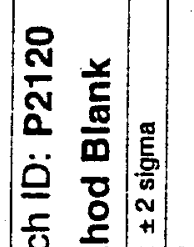

至

.

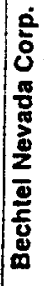

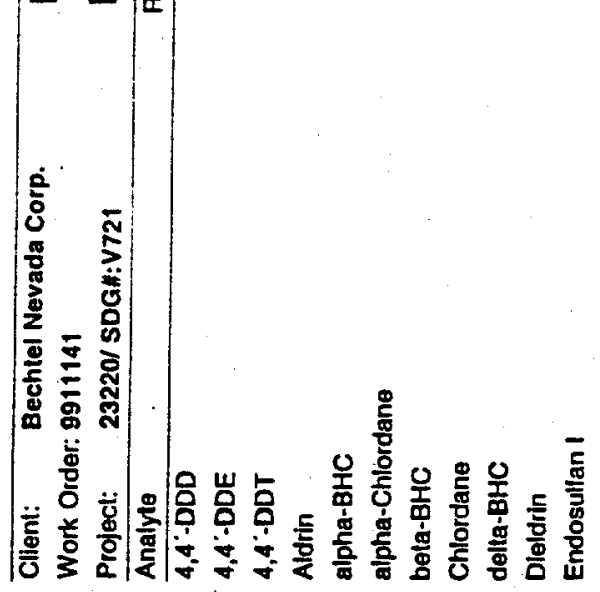


15000 W Gh Avenue Stuite 300 Golden, Colorado 80401.5047 (800)654.0506 (303) 277.1687 Fax(303) 277.1689

\begin{tabular}{llr}
\hline Endosullan II & ND & 0.04 \\
Endosullan sullale & ND & 0.7 \\
Endrin & ND & 0.06 \\
Endrin aldehyde & ND & 0.2 \\
Endrin kelone & ND & 0.1 \\
gamma-BHC & ND & 0.04 \\
gamma-Chlordane & NO & 0.06 \\
Heptachlor & ND & 0.03 \\
Heptachlor epoxide & ND & 0.1 \\
Methoxychlor & ND & 0.1 \\
Toxaphene & ND & 2
\end{tabular}

Surr: Decachlorobiphenyl

Surr: Tetrachloro-m-xylene

0.7
0.06
0.2
0.1
0.04
0.06
0.03
0.1
0.1
2
$116 \quad 18 \quad 125$

$96 \quad 23 \quad 110$
Qualiflers: ND - Not delecled at the reporting limil
$\mathrm{J}$ - Analyte detected below quantitation limits
E - Value above quantitation range

R - RPD outside accepted recovery limits

$X$ - Dupllcate sample(s) $<5$ times limil

S - Spike recovery outside accepted recovery limits
$Y$ - Unspiked sample $>4$ times amount spiked

B - Analyte detected in the associaled melhod blank

$Z$ - Sample $>10$ times blank result

Page 6 of 6

?


Job Number: 23220

Facillty 10: Nrs

Sampling Event: CAU 340
Cooler 10:

Cooler Temp:

Number of Coolers:

Cooler Units:

Logbook No.

Dellvery Order ID:

SEIR No.: CNJ 340-4-0

COC Number: cal 340-4

Laboratory: BARIwoR

sumpled 8y: JerRy $B_{\text {ren }}$

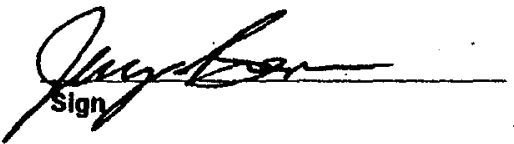

Print

Sign

Requested Analysis:

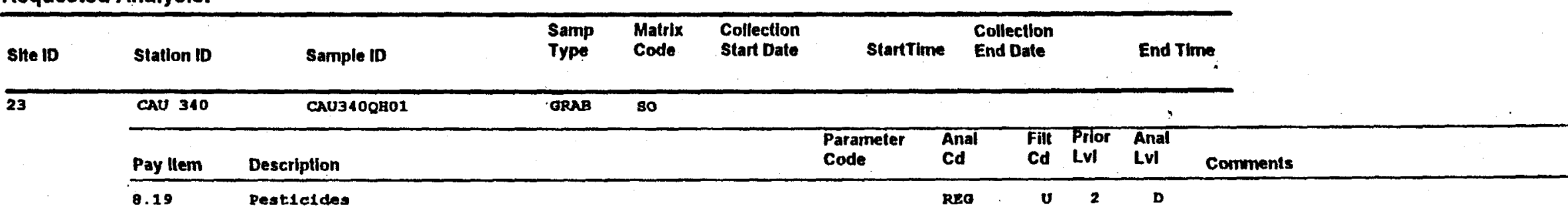

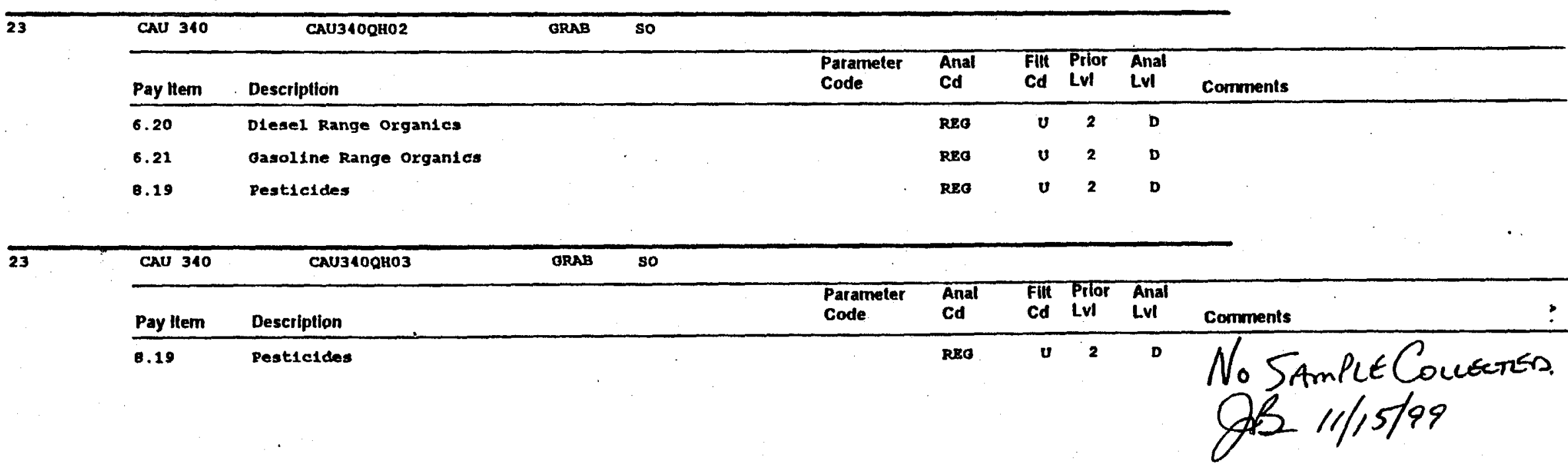

This package conforms to the conditions and limitations specified in 49 CFR 173.421 for excepled radioactive material, llmilted quantity, n.0.8., UN2910. 
Requested Analysis:

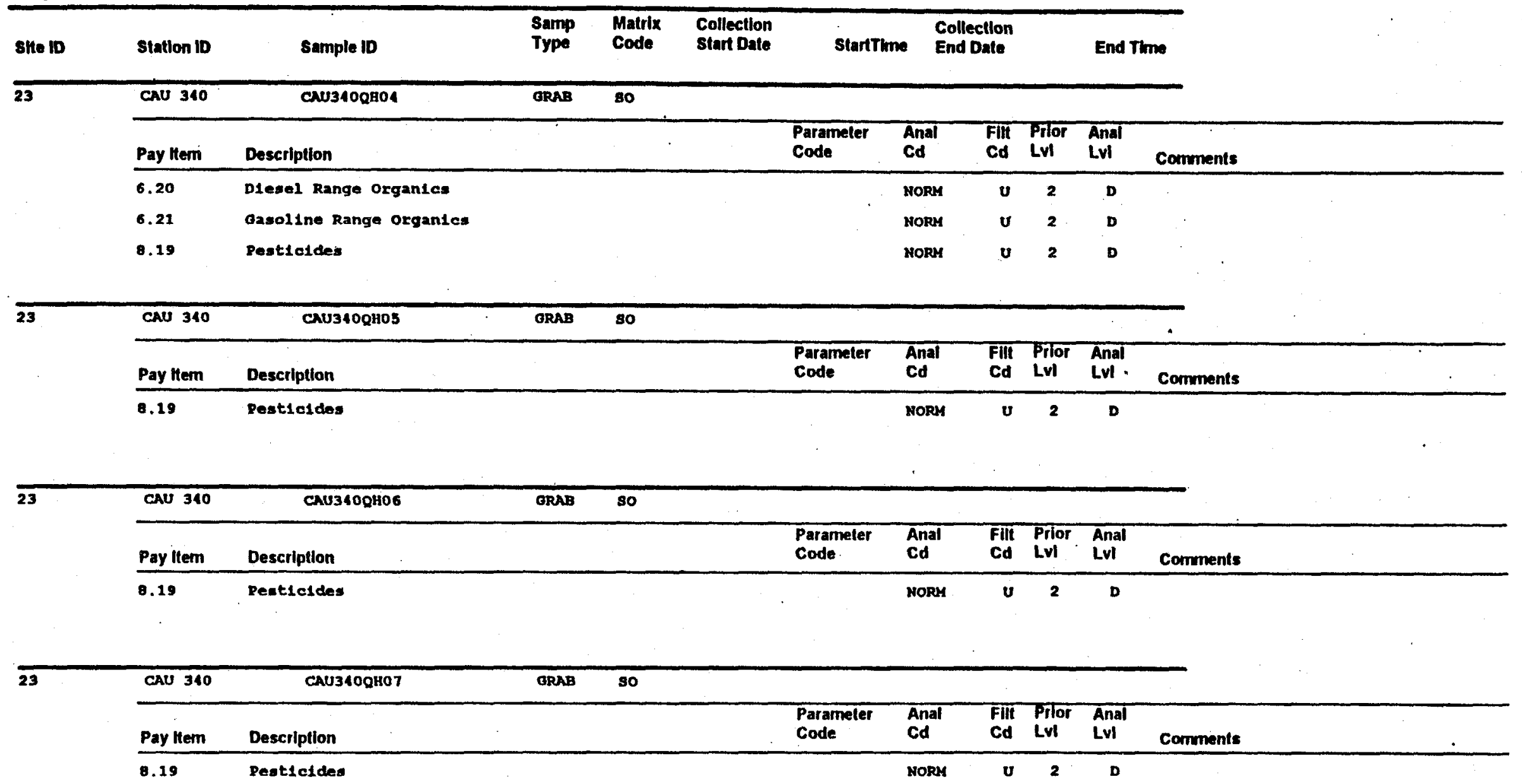

This package conforms to the conditions and limitations specined in 49 CFR 173.421 for excepled radioactive malental, limtled quanitly, n.o.s., UN2910. 
Requested Analysis:

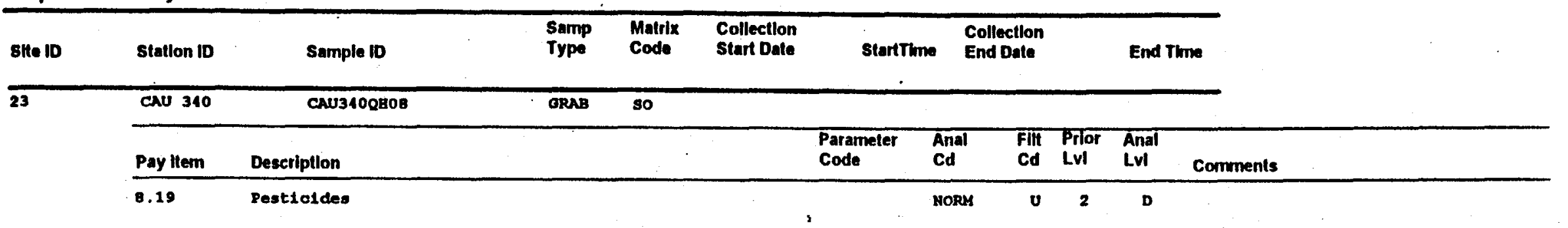

23

\begin{tabular}{|c|c|c|c|}
\hline CAU 340 & CAU340QH09 & ORAB & so \\
\hline
\end{tabular}

\begin{tabular}{|c|c|c|c|c|c|c|c|}
\hline Pay ltem & Description & $\begin{array}{l}\text { Parameter } \\
\text { Code }\end{array}$ & $\begin{array}{l}\text { Anal } \\
\text { Cd }\end{array}$ & $\begin{array}{l}\text { Fili } \\
\text { Cd }\end{array}$ & $\begin{array}{l}\text { Prlor } \\
\text { LvI }\end{array}$ & $\begin{array}{l}\text { Anal } \\
\text { LvI }\end{array}$ & Cormments \\
\hline 8.19 & Pesticldes & & NORY & $\mathbf{u}$ & 2 & D. & \\
\hline
\end{tabular}

23 CAU 340 CAU340Qk10

ORAB

so

\begin{tabular}{|c|c|c|c|c|c|c|c|}
\hline Pay ltem & Description & $\begin{array}{l}\text { Parameler } \\
\text { Code }\end{array}$ & $\begin{array}{l}\text { Anal } \\
\text { Cd }\end{array}$ & $\begin{array}{l}\text { Filt } \\
\text { Cd }\end{array}$ & $\begin{array}{l}\text { Prior } \\
\text { LvI }\end{array}$ & $\begin{array}{l}\text { Anal } \\
\text { LvI }\end{array}$ & Comments \\
\hline 0.19 & Pest1c1des & & NORH & v & 2 & D & \\
\hline
\end{tabular}

23

\begin{tabular}{llll}
\hline CAU 340 & CAU34OQH11 & GRAB & SO \\
\hline
\end{tabular}

Pay liem Description

so

8.19 Pesticides

Parameter

Paramele
Code

\begin{tabular}{l}
\hline $\begin{array}{l}\text { Anal } \\
\text { Cd }\end{array}$ \\
\hline NORM
\end{tabular}

Cd LvI LvI Comments
Lul


Requested Analysis:

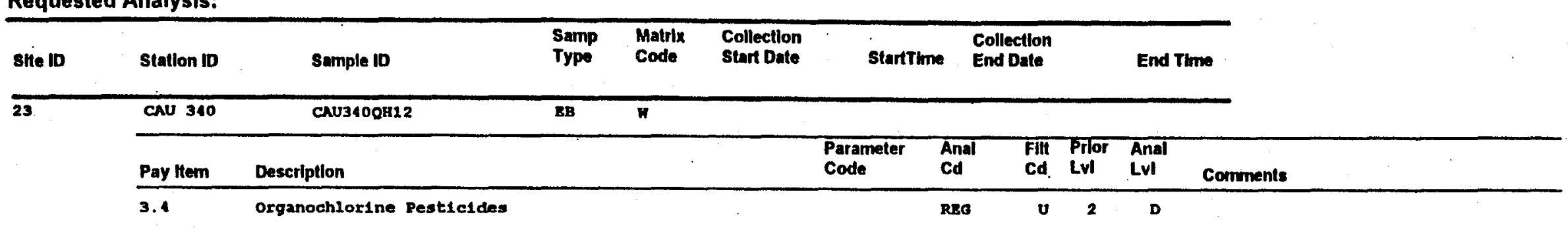

\begin{tabular}{|c|c|c|c|c|c|c|c|c|c|c|}
\hline \multirow[t]{3}{*}{23} & CAU 340 & CAU340QH13 & FRP & so & \multirow[b]{2}{*}{$\begin{array}{l}\text { Parameter } \\
\text { Code }\end{array}$} & \multirow[b]{2}{*}{$\begin{array}{l}\text { Anal } \\
\text { Cd }\end{array}$} & \multirow[b]{2}{*}{$\begin{array}{l}\text { Filt } \\
\text { Cd }\end{array}$} & \multirow[b]{2}{*}{$\begin{array}{l}\text { Pror } \\
\text { Lul }\end{array}$} & \multirow[b]{2}{*}{$\begin{array}{l}\text { Anal } \\
\text { LvI }\end{array}$} & \multirow[b]{2}{*}{ Comments } \\
\hline & Pay Hem & Description & & & & & & & & \\
\hline & $\begin{array}{l}6.20 \\
6.21 \\
6.19\end{array}$ & $\begin{array}{l}\text { Dlesel Range Organios } \\
\text { Gasollne Range Organics } \\
\text { Pest1cldes }\end{array}$ & & & & $\begin{array}{l}\text { ReO } \\
\text { REO } \\
\text { REO }\end{array}$ & $\begin{array}{l}\mathbf{v} \\
\mathbf{v} \\
\mathbf{v}\end{array}$ & $\begin{array}{l}2 \\
2 \\
2\end{array}$ & $\begin{array}{l}\text { D. } \\
\text { D } \\
\text { D }\end{array}$ & \\
\hline \multirow[t]{3}{*}{23} & CAU 340 & CAU34OQH1 & FRP & so & & & & & . & \\
\hline & Pay Hem & Description & & & $\begin{array}{l}\text { Parameter } \\
\text { Code }\end{array}$ & $\begin{array}{l}\text { Anal } \\
\text { Cd }\end{array}$ & $\begin{array}{l}\text { Fili } \\
\text { Cd }\end{array}$ & $\begin{array}{l}\text { Prlor } \\
\text { LvI }\end{array}$ & $\begin{array}{l}\text { Anai } \\
\text { LvI }\end{array}$ & Comments \\
\hline & 0.19 & peaticldes & & & & REa & $\mathbf{u}$ & 2 & D & \\
\hline
\end{tabular}

\begin{tabular}{|c|c|c|c|c|c|c|c|c|c|c|c|}
\hline \multirow[t]{3}{*}{23} & CAU 340 & CAU340QH15 & Ms & so & & & & & & & \\
\hline & Pay Item & Description & & & $\begin{array}{l}\text { Parameter } \\
\text { Code }\end{array}$ & $\begin{array}{l}\text { Anal } \\
\text { Cd }\end{array}$ & $\begin{array}{l}\text { Fill } \\
\text { Cd }\end{array}$ & $\begin{array}{l}\text { Prlor } \\
\text { LVI }\end{array}$ & $\begin{array}{l}\text { Anal } \\
\text { LvI }\end{array}$ & Comments & . \\
\hline & 0.19 & Pesticides & & & & NORM & $\mathbf{u}$ & 2 & D & MS - take tron ample 06 & \\
\hline
\end{tabular}

Thls package conforms to the conditlons and limliations specined In 49 CFR 173.421 for excepted radloaclive matertal, limilled quantity, n.0.s., UN2910. 
11 109/1990

NTS - PRODUCTION

CHAIN OF CUSTODY RECORD: CAU 340-4OOPE

Requested Analysis:

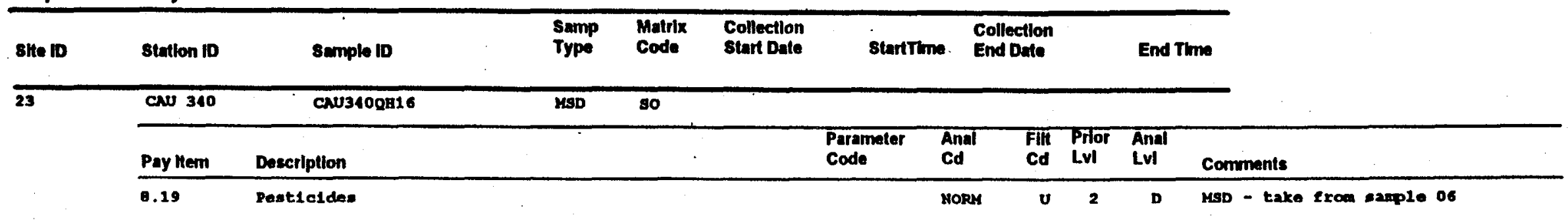


Containers Included on C.o.C.:

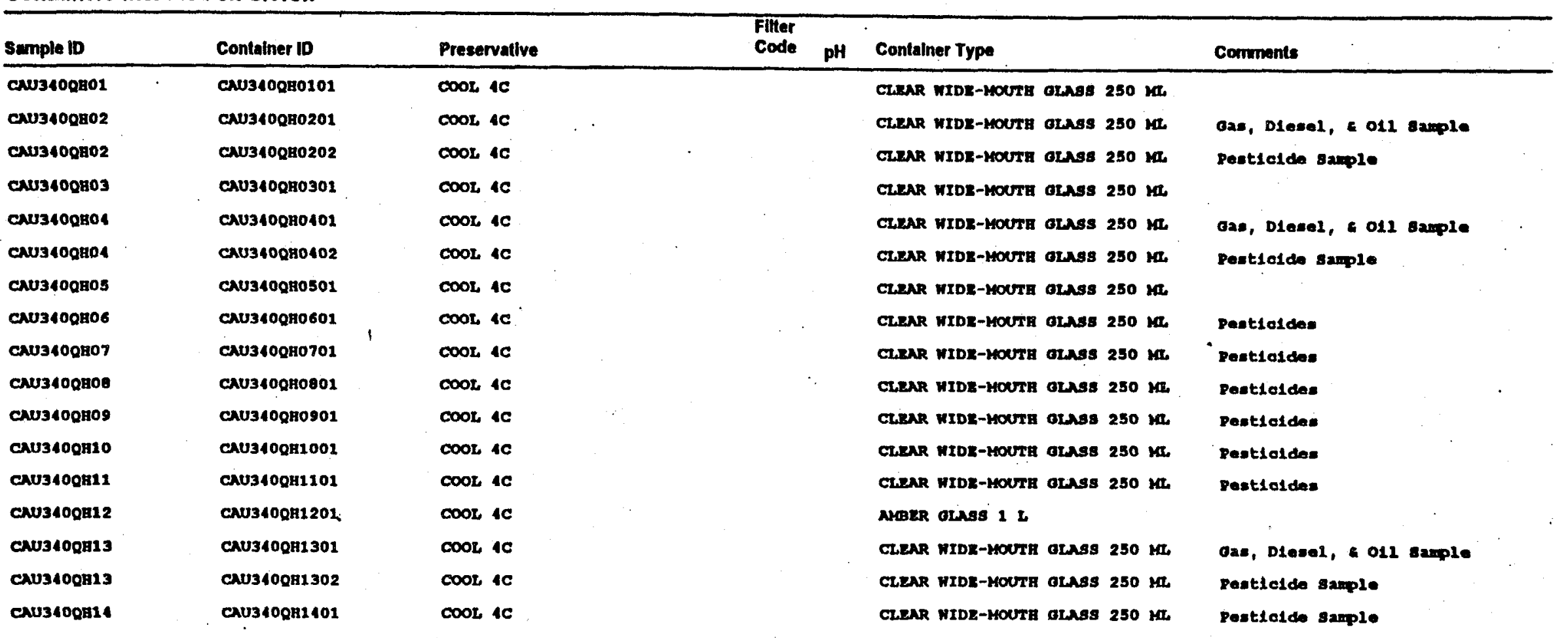

This package conforms to the condilions and fimitations specified in 49 CFR 173.421 for excepted radioactive materlal, limlted quantity, n.0.8., UN2910. 
Transfer Information:

Rellnqulshed By

$$
\text { Recelved By }
$$

Transfer Daterthe

Reason

Trainc Report No.

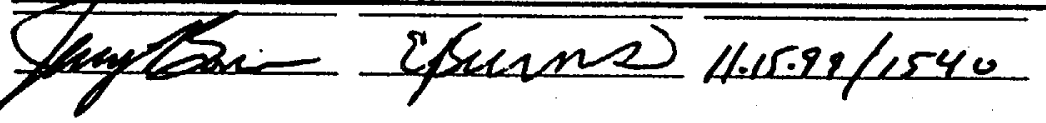

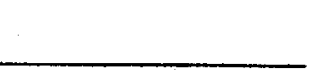

$+$

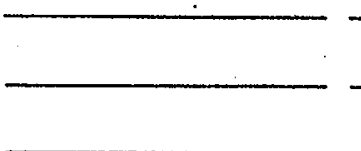

Cormments:

Potentlal Contamination Yes No

Radiological

Chemical

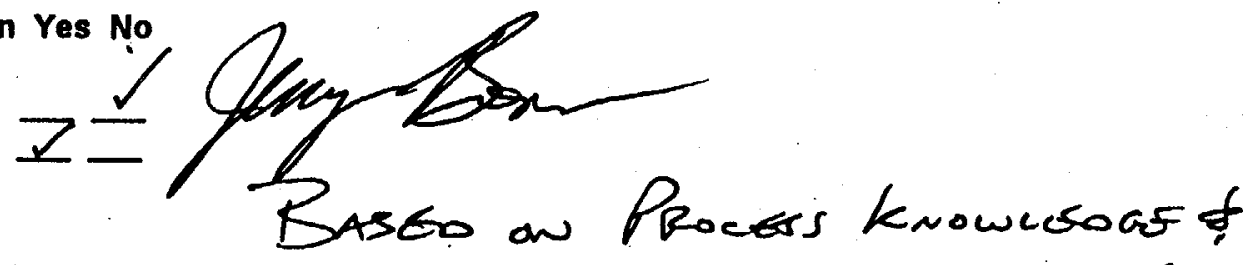

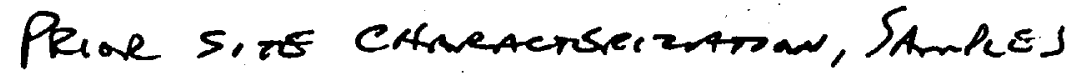

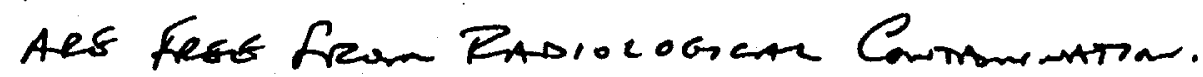

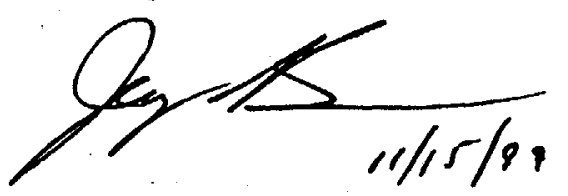



Barringer Laboratories, Inc.

I5000 W 6th Avenue Suite $300 \quad$ Golden. Colorado 80401-5047

(800) 654-0506 (303) 277.1687 Fax (303) 277.1689

Ted Redding

Bechtel Nevada Corp.

P.O. Box 98521

MS NTS 273

Las Vegas, NV 89193-8521 .

Phone: 1-702-295-7220

Fax: 1-702-295-4773

Work Order: 9911154

Project: SDG\#V723. DO W1762, job 23220, SEIR CAU 340

Dear Ted Redding,

Barringer Laboratories received 15 samples on $11 / 18 / 99$ for the analyses presented in the following report.

There were no problems with the analyses and all data for associated QC met EPA or laboratory specifications except where noted in the Case Narrative.

If you have any questions regarding these test results, please feel free to call.
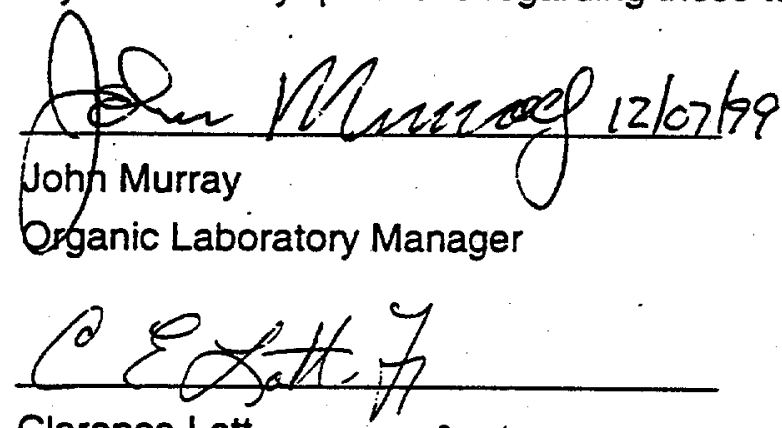

Clarence Lott

Project Review 1 if $7 / 99$ 


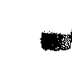


Date: 07-Dec-99

\section{Barringer Laboratories, Inc.}

I5000 W 6th Avenue Suite 300 Golden, Colorado s0401-5047 (800)6.54-0506 (303) 277.J687 Fax (303) 277.1689

Client: Bechtel Nevada Corp.

Project: $\quad$ SDG\#V723. DO W1762, job 23220, SEIR

Work Order: 9911154

SAMPLE SUMMARY

Date Received: $11 / 18 / 99$

Temp Received: $4^{\circ} \mathrm{C}$

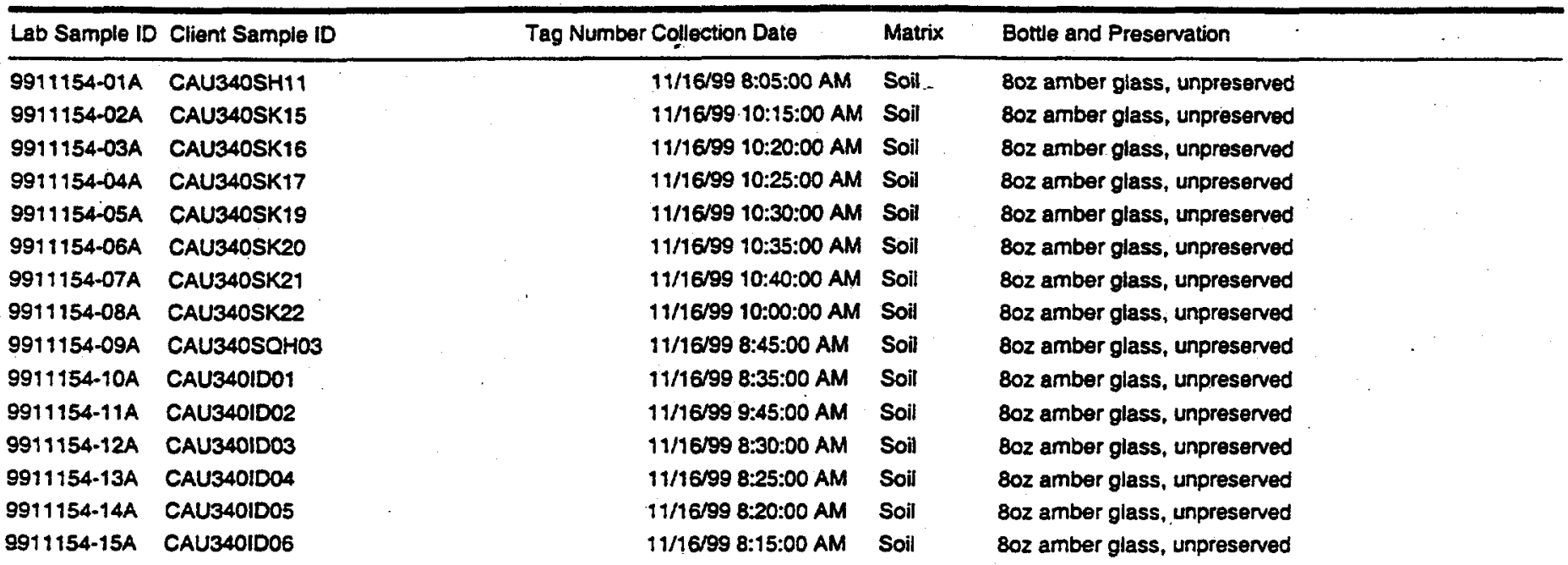




\begin{tabular}{lll}
\hline \hline Client: & Bechtel Nevada Corp. & \\
Project: & SDG\#V723. DO W1762, job 23220, SEIR & CASE NARRATIVE \\
Work Order: & 9911154 & \\
\hline
\end{tabular}

All reported values in this report have been rounded to the correct number of significant figures. All calculations have been performed before applying significant figures, thereiore, not all calculations may be reproducible with the results printed in this report.

Analytical Comments for method SW846 8081A: There are some analytes that are being reported as estimated due to the analyte being above our calibration curves linear range. The client is aware of this and does not want the samples analyzed at the required dilution to bring all analytes within the calibration range.

This report has been amended from the report dated $11 / 30 / 1999$ to include the dilutions of the samples that were previously reported with estimated results for method SW846-8081A. 
Date: 07-Dec-99 Barringer Laboratories, Inc. CLIENT SAMPLE REPORT

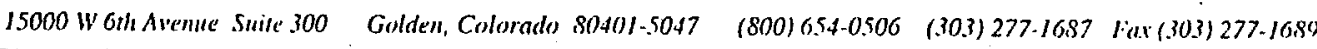

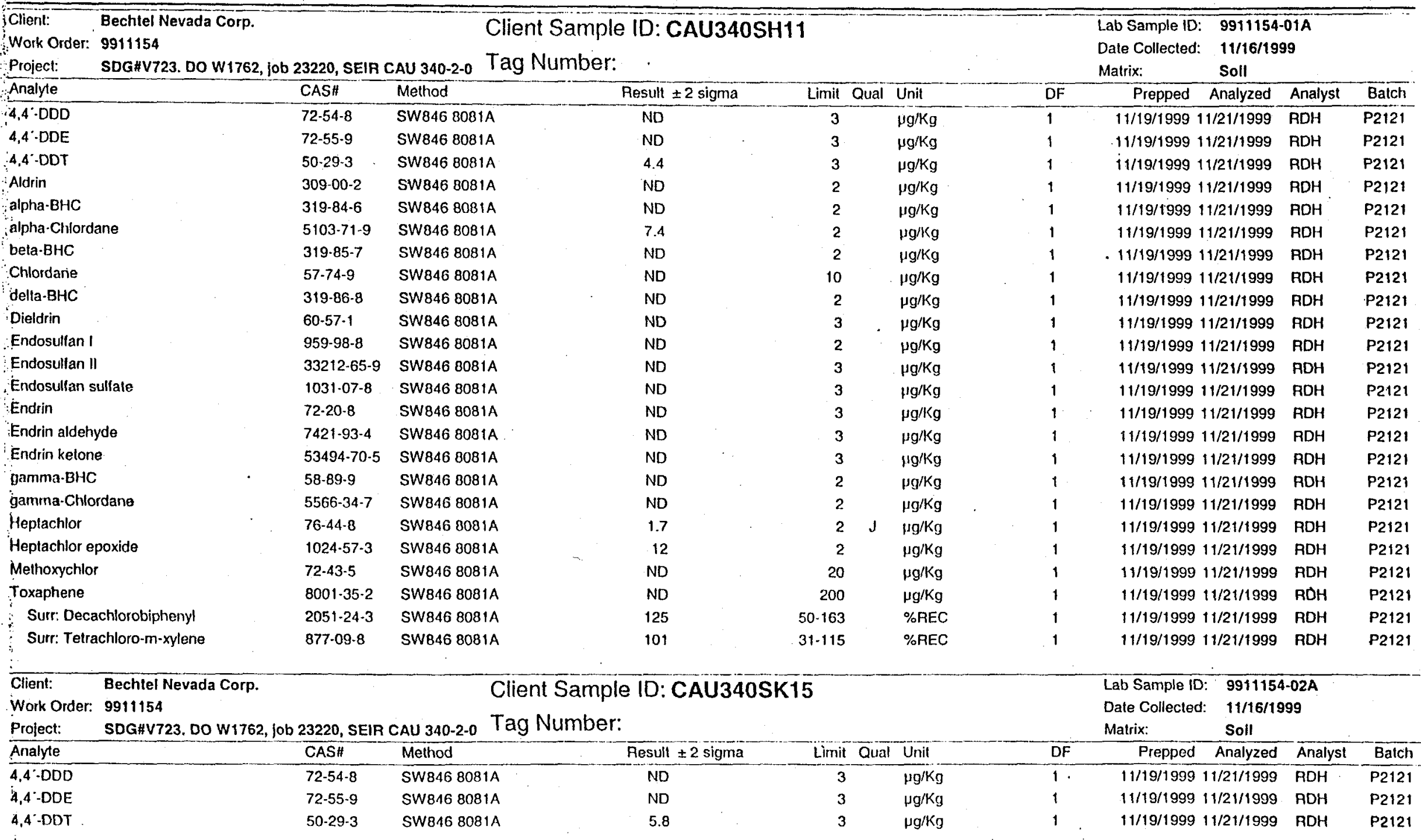

NO - Not detected at the reporting limit

$R \cdot R P D$ outside accepted recovery limits

$Y$ - Unspiked sample $>4$ times amount spiked

J - Analyte delected below quantilation limils

$X$ - Duplicate sample(s) $<5$ times limit

$B$ - Analyte detected in the associated method blank

$E$ - Value above quantitation range

$S$ - Spike recovery outside accepted recovery linits

$Z$ - Sample $>10$ times blank result 
Date: 07-Dec-99 Barringer Laboratories, Inc.

CLIENT SAMPLE REPORT 15000 W Gih Aveme Suive 300 Golden, Colorado 80401.5047 (800)654-0.506 (.303) 277.16.87 Fax (.30.3) 277-1689

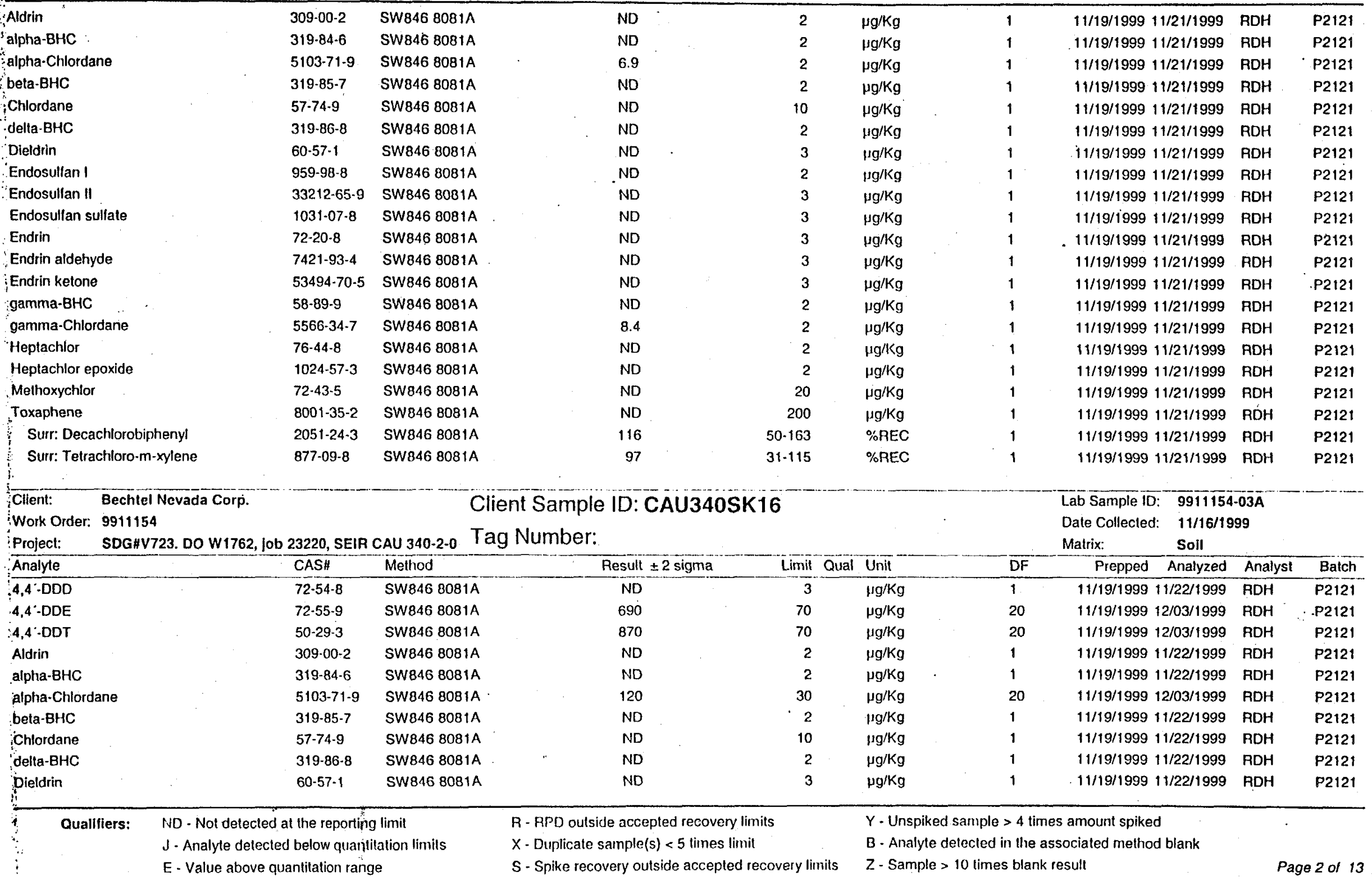


Dale: $07-$ Dec-99 Barringer Laboratories, Inc.

\section{CLIENT SAMPLE REPORT}

15000 W 6th Avenue Suite 300 Golden, Colorado 80401-5047 $\quad$ (800) 654-0506 (303) 277.1687 Fax (30.3) 277.1689

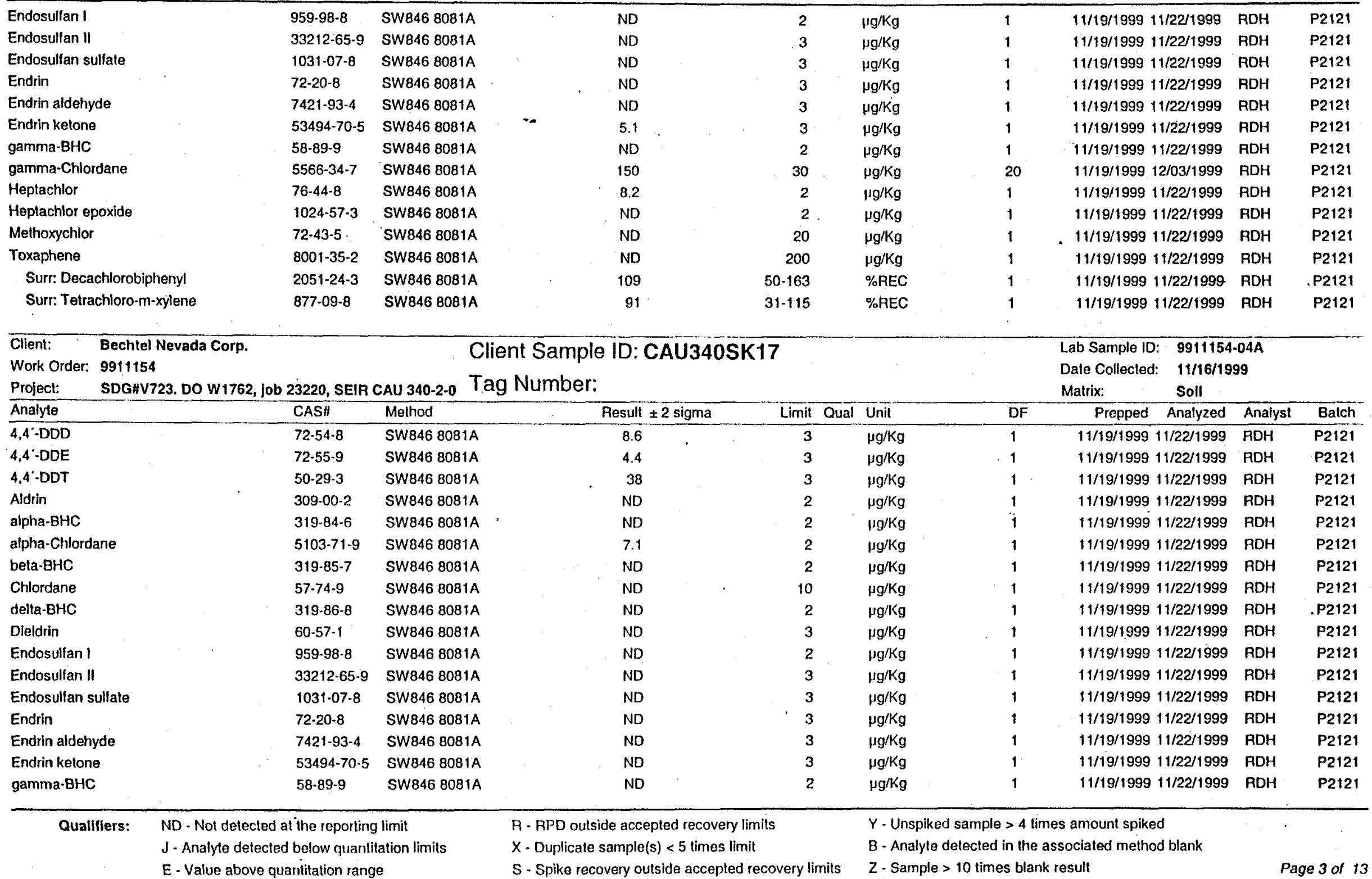


Date: 07-Dec-99 Barringer Laboratories, Inc.

\section{CLIENT SAMPLE REPORT}

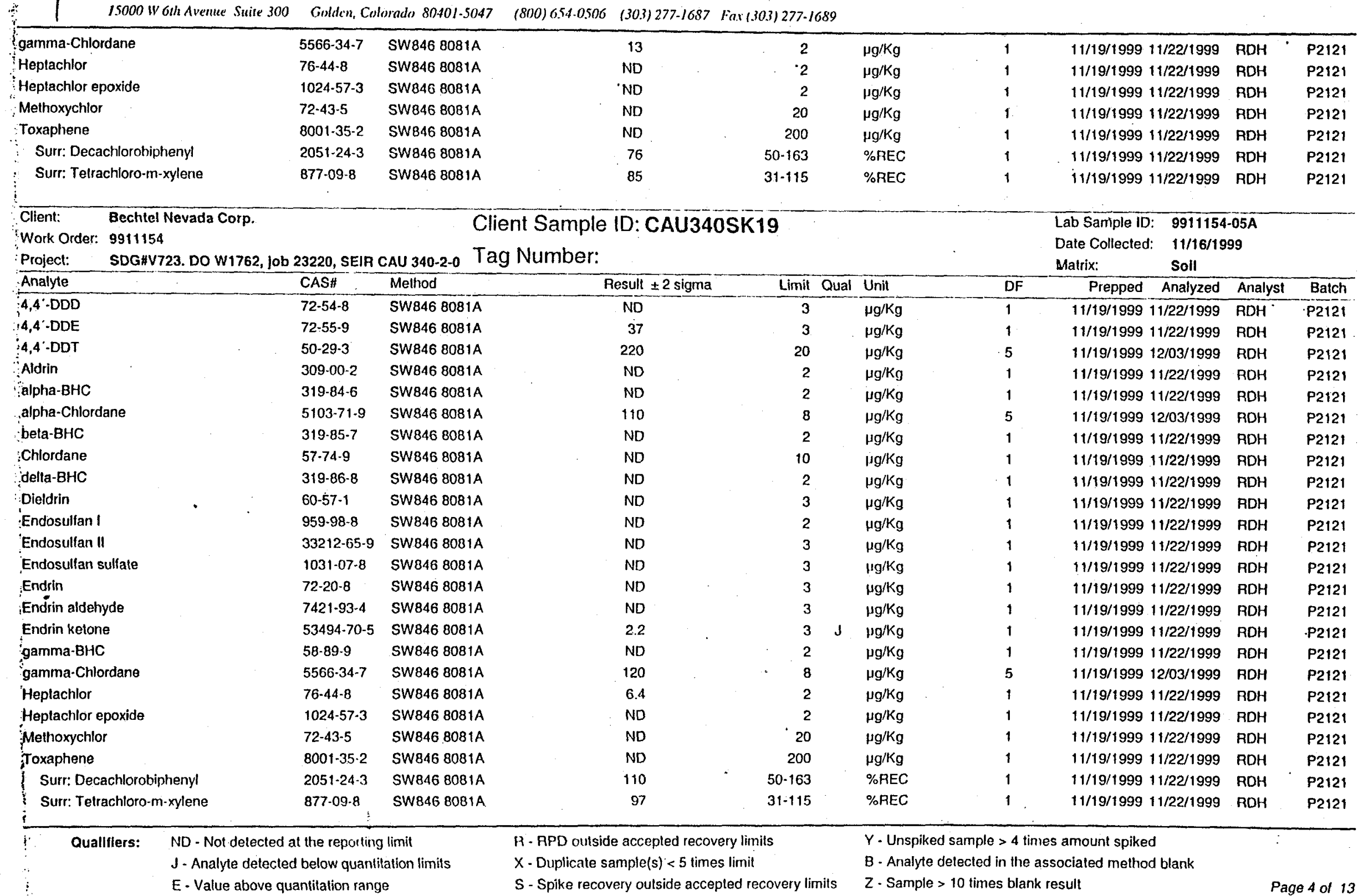

$Z$ - Sample $>10$ times blank result 


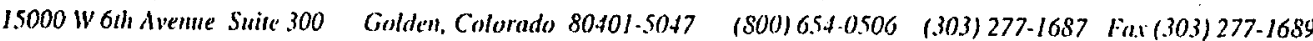

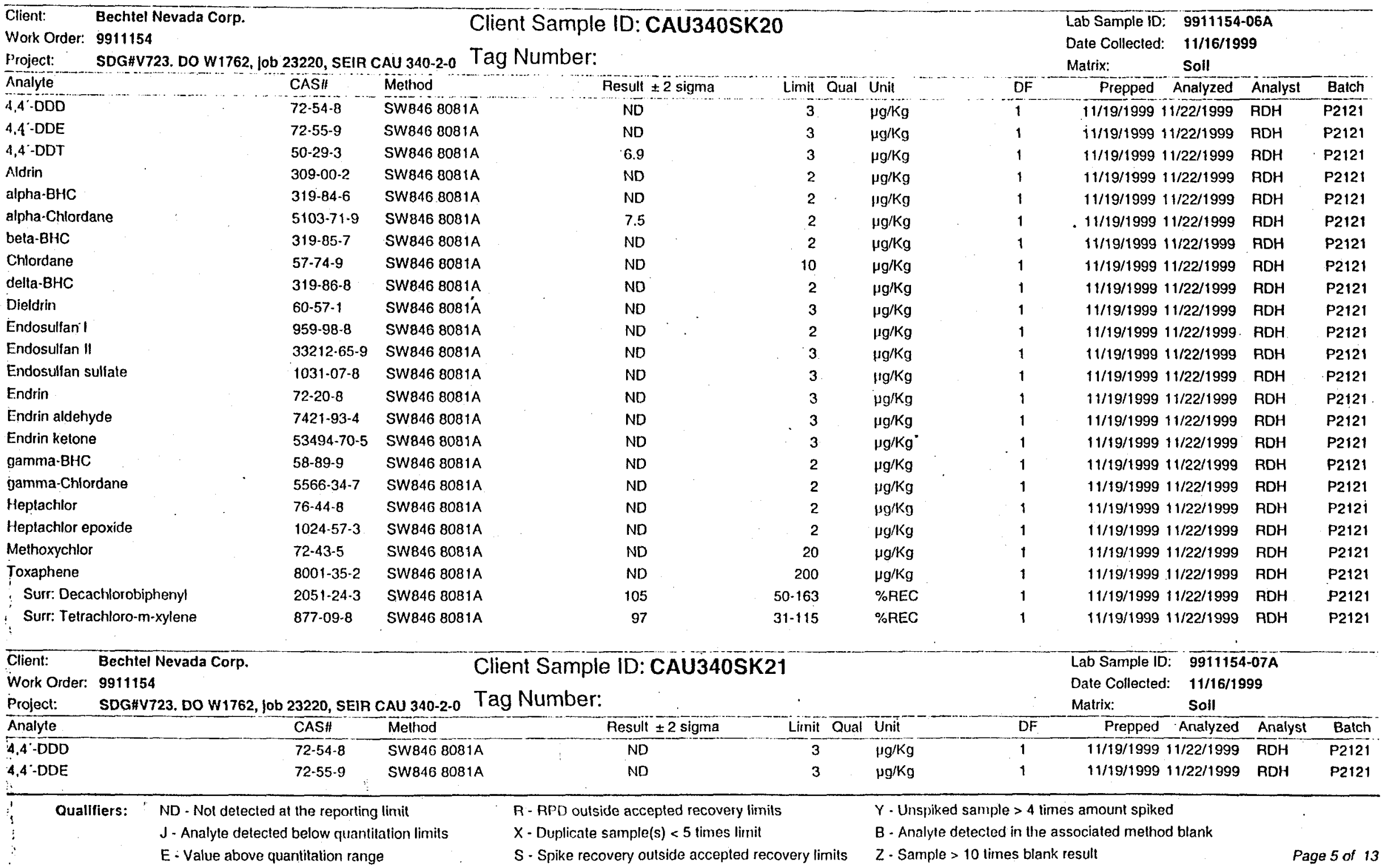


Dale: 07-Dec-99 Barringer Laboratories, Inc.

CLIENT SAMPLE REPORT $\begin{array}{lllll}15000 \text { w 6th Avemue Suite } 300 \quad \text { Golden, Colorado 80401-5047 } & \text { (800) 0.54.0506 (30.3) 277-1687 Fax (30.3) 277-1689 }\end{array}$

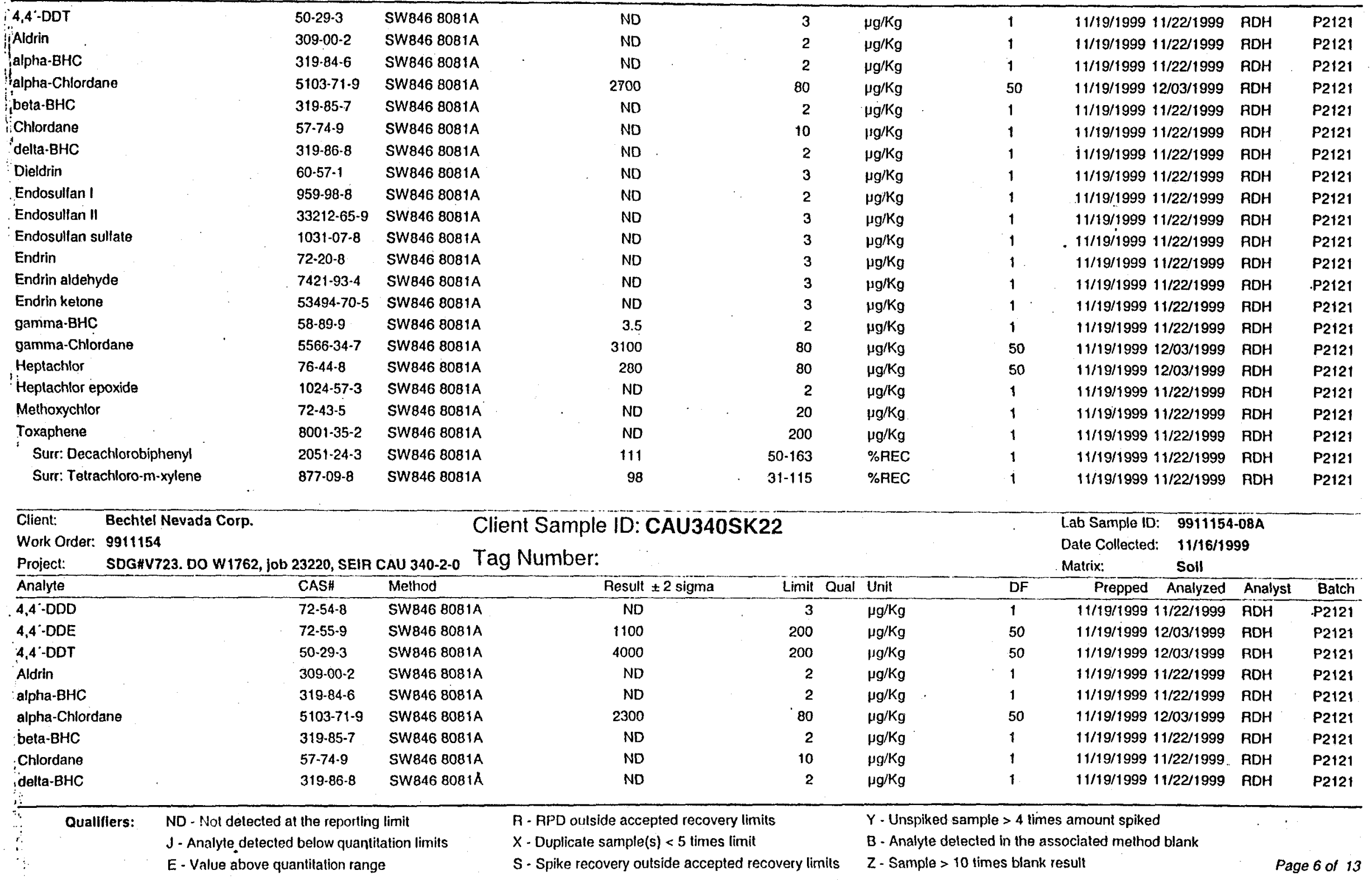


Date: $07-$ Dec-99 Barringer Laboratories, Inc.

\section{CLIENT SAMPLE REPORT}

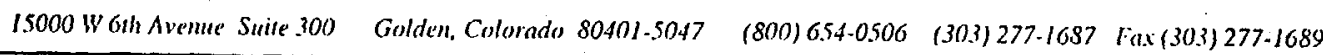

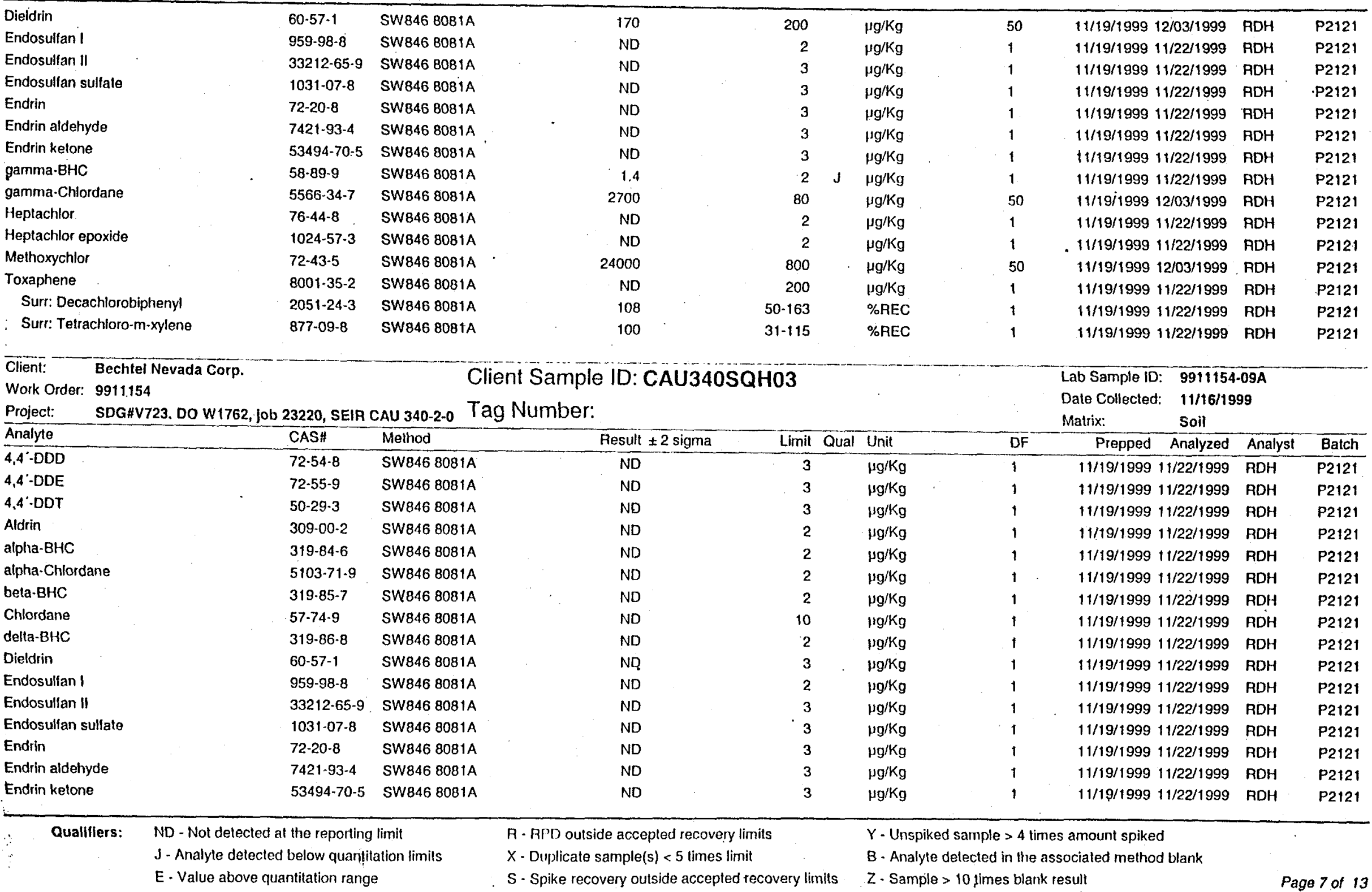




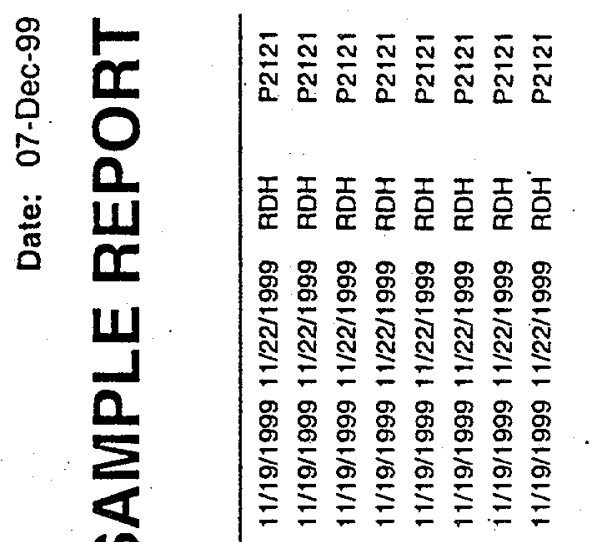

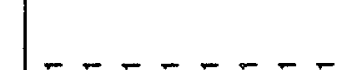

点

$\div$

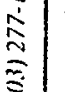

$\sin n 888$

$\frac{0}{8} \frac{1}{\circ}$

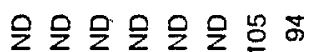

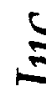

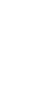

辛

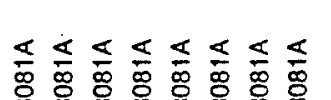

○ :

$\%$ L $0 \% 5 \%$

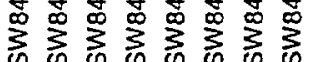

$\Xi$

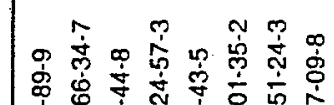

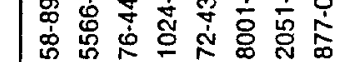

气 
Date: 07:Dec-99

Barringer Laboratories, Inc.

CLIENT SAMPLE REPORT

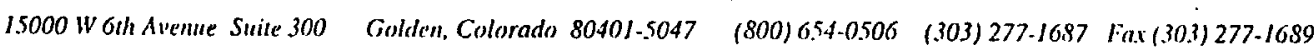

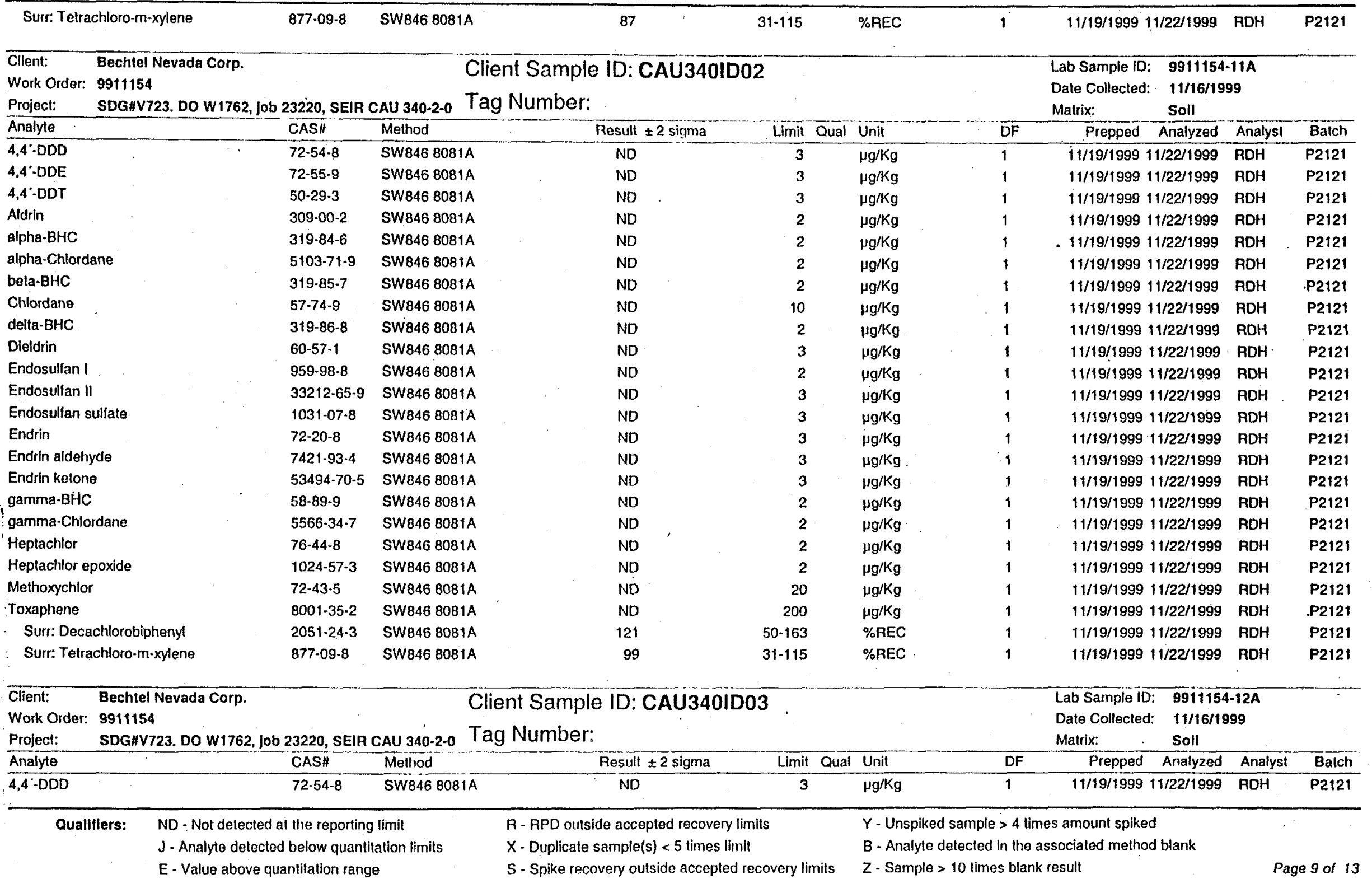


Date: $07-\mathrm{Dec}-99$ Barringer Laboratories, Inc.

CLIENT SAMPLE REPORT

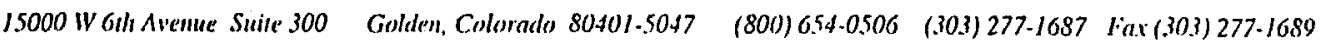

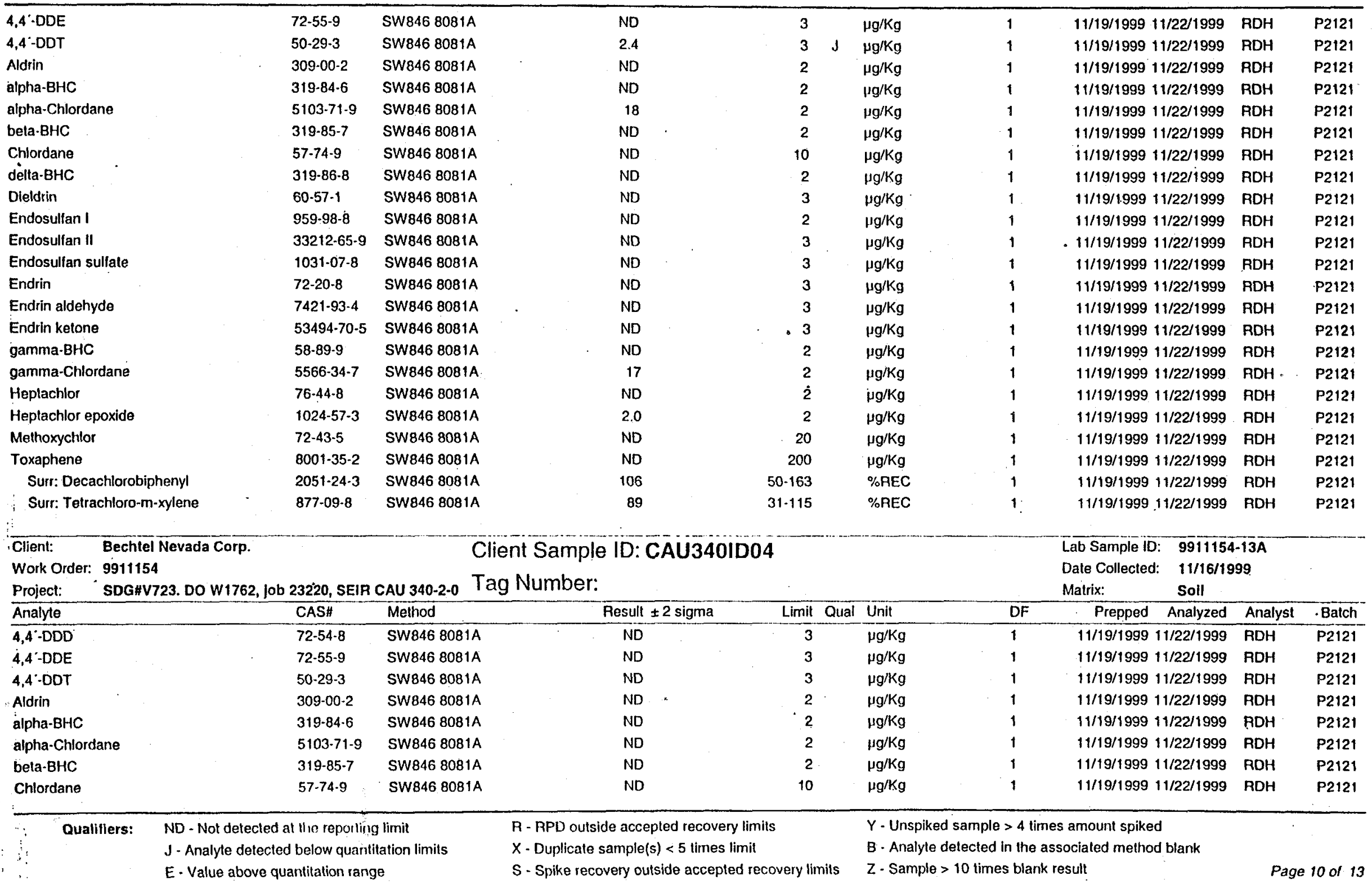


Date: 07-Dec-99 Barringer Laboratories, Inc.

CLIENT SAMPLE REPORT

15000 w G1h Alenue Suite 300 Golden, Colorado 80401-5047 $\quad$ (800) 654-0506 (303) 277.1687 Fax (30.3) 277-1689

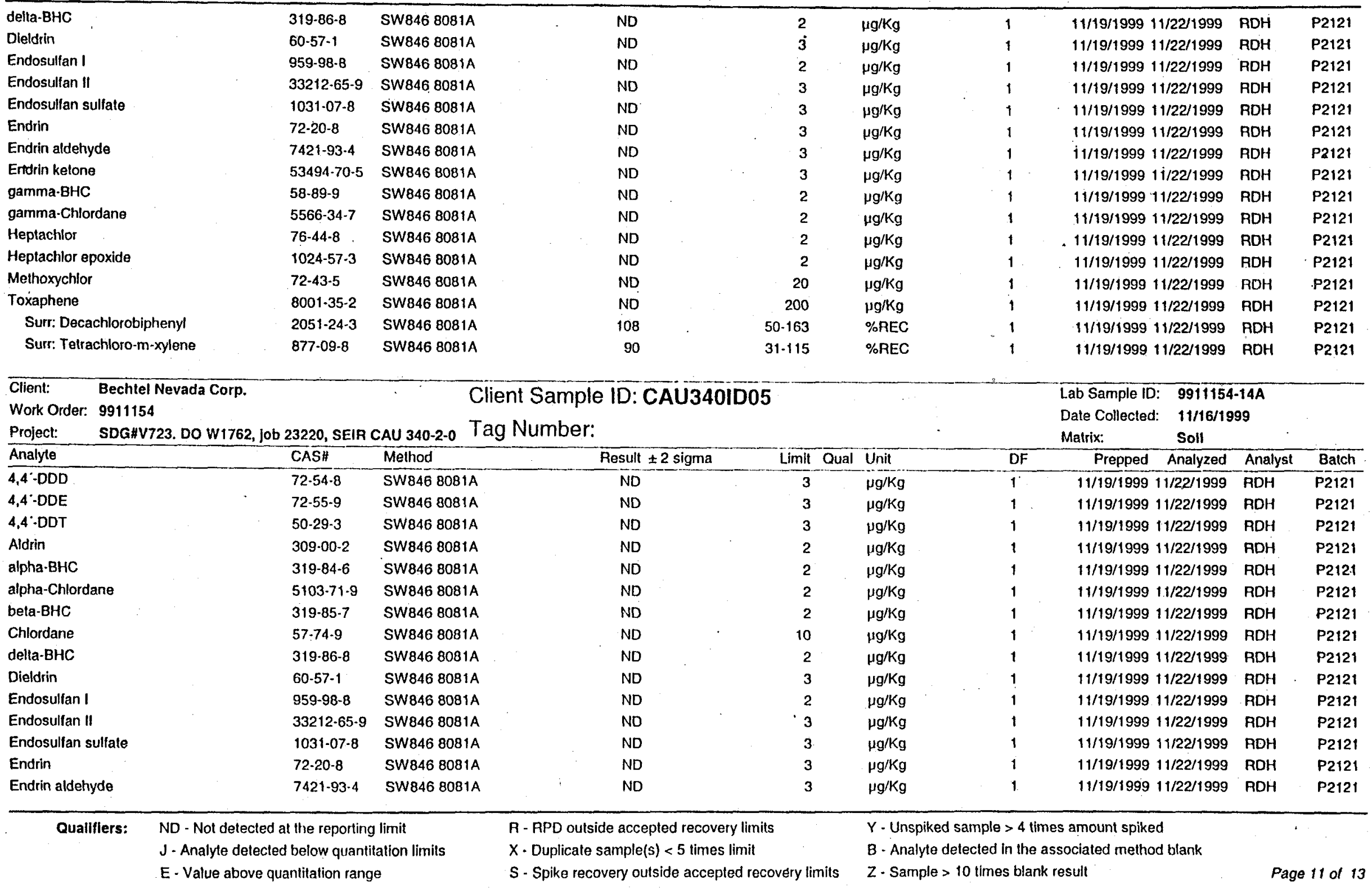


Date: 07-Dec-99

Barringer Laboratories, Inc.

CLIENT SAMPLE REPORT

15000 W Gth Avemue Suite 300 Golden. Colorado 80401.5047 (800)6.54.0506 (303) 277.1687 Pax (30.3) 277.1689

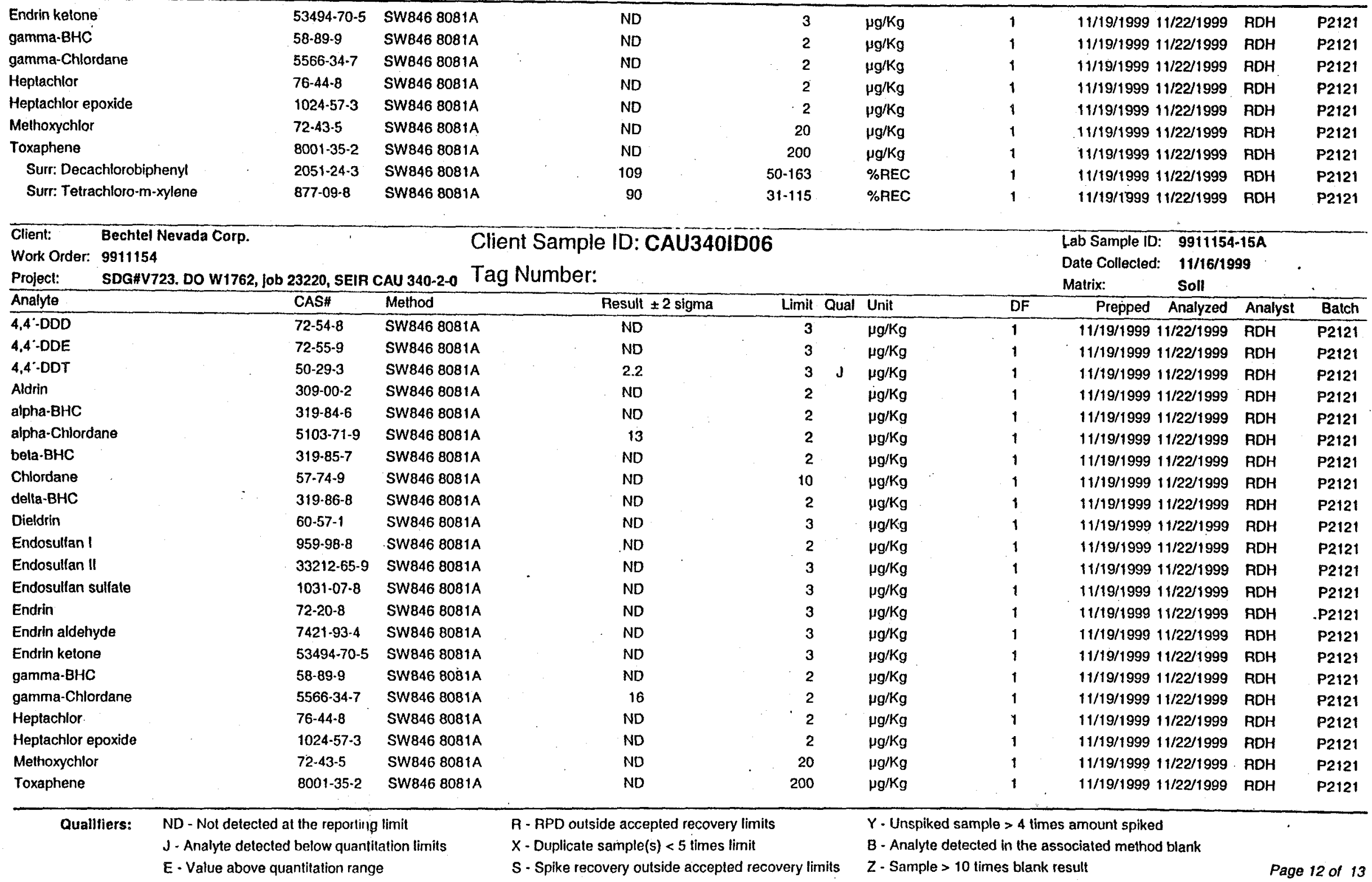

$\begin{array}{lll}\text { J. Analyte detected below quantilation limils } & X \text {-Duplicate sample(s) }<5 \text { times limit } & B \text { - Analyte detected in the associa } \\ E \text { - Value above quantitation range } & S \text { - Spike recovery outside accepted recovery limits } & Z \text { - Sample }>10 \text { times blank result }\end{array}$ 


\section{CLIENT SAMPLE REPORT}

15000 W Gth Avemue Suite .300

Golden, Colurndo 80401.504 (800) $0.54 .0506 \quad$ (.30.3) 277.1687 Fax (30.3) 277.1689 

15000 W 6th Avenue Suite 300 Golden, Colmado 80401-5047 (800) 6.54.0506 (30.3) 277-1687 Fa.r(.303) 277.1689

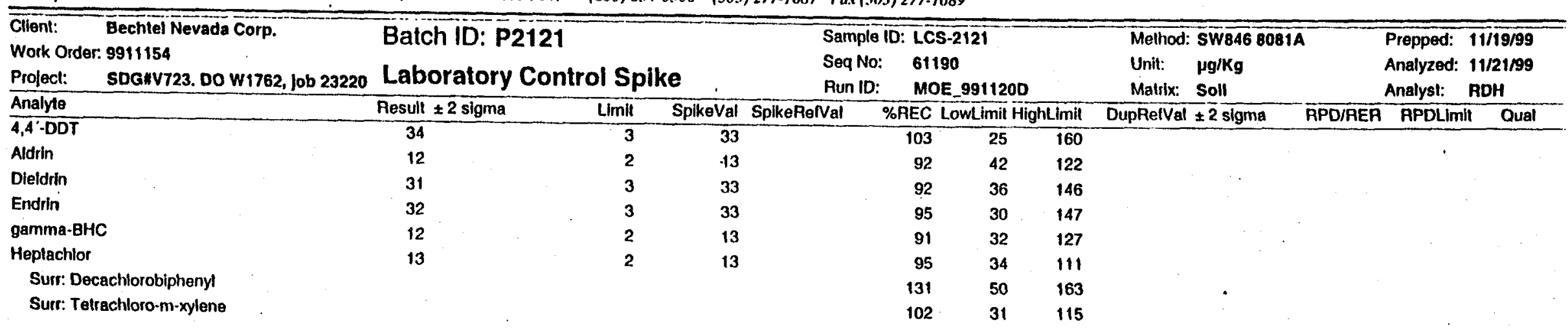

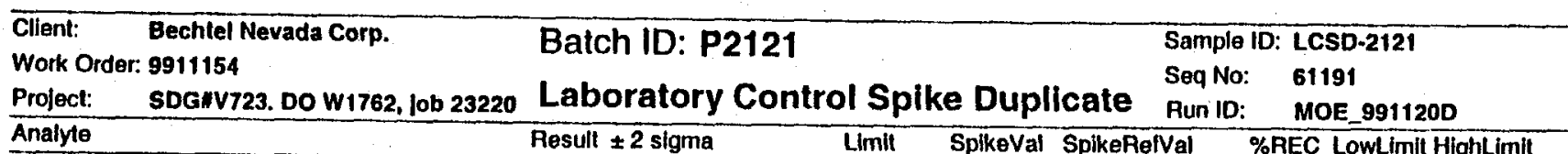

\section{Prepped: 11/19/99} Analyzed: 11/21/99

\begin{tabular}{lrcrrrrr}
\hline Analyle & Result \pm 2 sigma & Limit & SplkeVal SpikeRelVal & \%REC LowLimit HighLimit Dup \\
\hline $4,4^{\circ} \cdot \mathrm{DDT}$ & & 25 & 3 & 33 & 76 & 25 & 160
\end{tabular}

Aldrin

Dleldrin

Endrin

gamma-BHC

Heplachlor

10

26

26

3

3

Sur. Decachlorobiphenyl

$\begin{array}{rrr}78 & 42 & 12 \\ 78 & 36 & 146 \\ 78 & 30 & 147 \\ 79 & 32 & 127 \\ 79 & 34 & 11 \\ 112 & 50 & 163 \\ 90 & 31 & 115\end{array}$

\begin{tabular}{lcc} 
Method: SW846 6081A \\
$\begin{array}{l}\text { Unit: } \\
\text { Malrix: }\end{array} \begin{array}{l}\mu g / \mathrm{Kg} \\
\text { Soll }\end{array}$ \\
\hline 160 & 34 \\
122 & 12 \\
146 & 31 \\
147 & 32 \\
127 & 12 \\
111 & 13 \\
163 & & \\
115 & &
\end{tabular}

Surr: Telrachloro-m·xylene

Sample ID: MBLK-2121

Seq No: 61189

Run ID: MOE_991120D

Melhod: SWB46 B081A

Unit: $\quad \mu g / K g$

Matrix: Soll

Analyst: $\mathrm{ADH}$

$\begin{array}{crc}30 & 20 & \text { A }\end{array}$

3020

16
17

1920

$14 \quad 20$

$18 \quad 20$

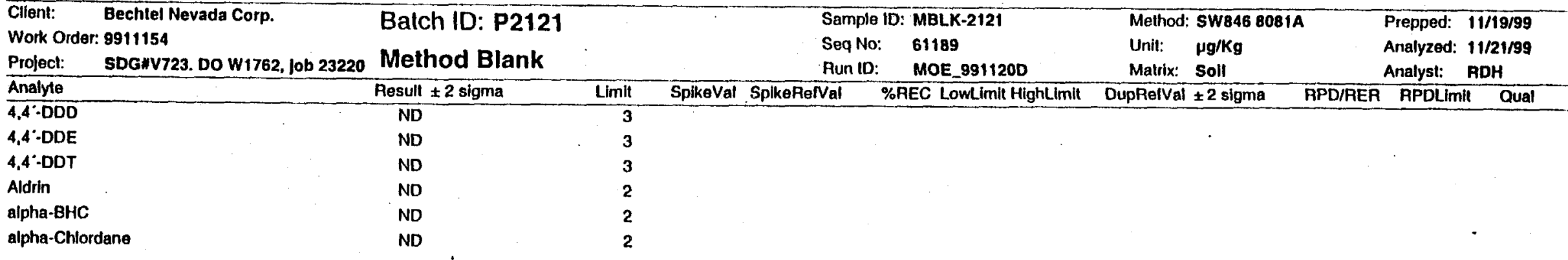

Quallilers: ND - Not detected at the reporting limlt

$\mathrm{J}$ - Analyte detected below quantitation limits

A - RPD outside accepted recovery limils

$Y$ - Unspiked sample $>4$ limes amount spiked

$E$ - Value above quantitation range

$X$ - Duplicate sample(s) $<5$ times limit

$B$ - Analyte delecled in the associated melhod blank

S - Spike recovery outside accepted recovery limils

$Z$ - Sample > 10 times blank tesult 
$\begin{array}{llllll}15000 \text { W Gth Avenue Suile } 300 \text { Golden, Colorado 80401-5047 } & \text { (800) 6.54-0506 (.303) 277-1687 Fa.r (30.3) 277-1689 }\end{array}$

\begin{tabular}{|c|c|}
\hline beta-BHC & ND \\
\hline Chlordane & ND. \\
\hline delta-BHC & ND \\
\hline Dieldrin & ND \\
\hline Endosulian I & ND \\
\hline Endosullan II & ND \\
\hline Endosulian sullale & No \\
\hline Endrin & NO \\
\hline Endrin aldehyde & ND \\
\hline Endrin ketone & No \\
\hline gamma-BHC & ND \\
\hline gamma-Chlordane & NO \\
\hline Heptachlor & ND \\
\hline Heptachlor epoxide & ND \\
\hline Melhoxychlor & NO \\
\hline Toxaphene & No \\
\hline
\end{tabular}

Surr: Docachlorobiphenyl

Surr: Tetrachloro-m-xylene

$128.50 \quad 163$

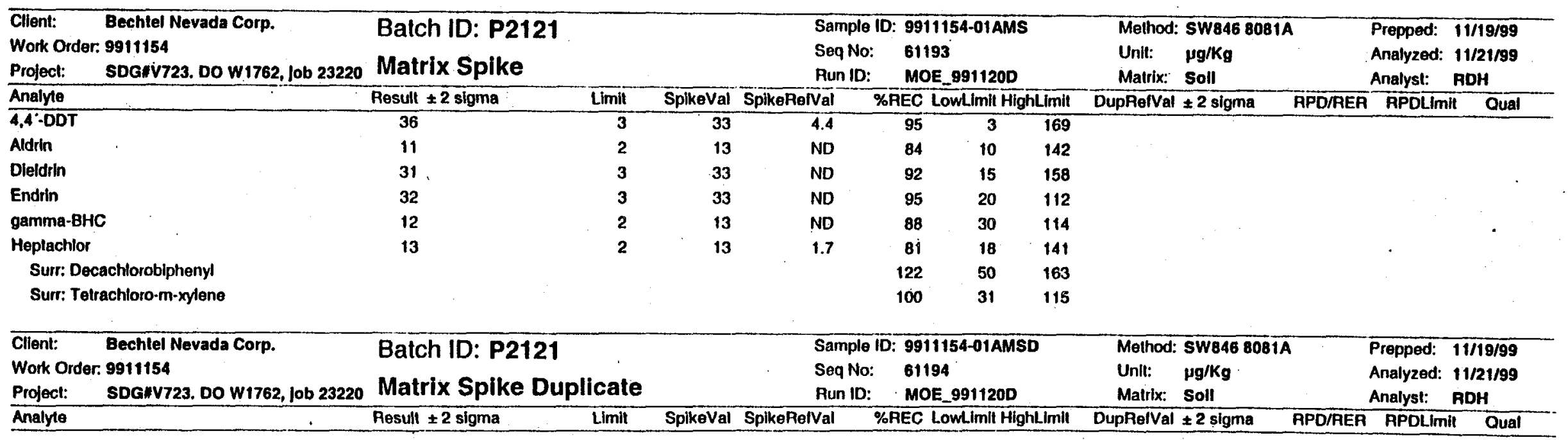

Qualliters: ND - Not delected al the reporting limit

$\mathrm{J}$ - Analyte detected below quantitation limits

E - Value above quantilation range
R - RPD outside accepted recovery limits

$X$ - Duplicate sample(s) $<5$ limes limit

S - Splke recovery outside accepted recovery limits
$Y$ - Unspiked sample > 4 times amount spiked

B - Analyte detected in the associated method blank

Z - Sample > 10 times blank resull 


\section{Barringer Laboratories, Inc.}

Date: 07-Dec-99

15000 W 6th Avesue Suite 300 Golden, Colourado 80401.5047

\section{BATCH QC SUMMARY REPORT}

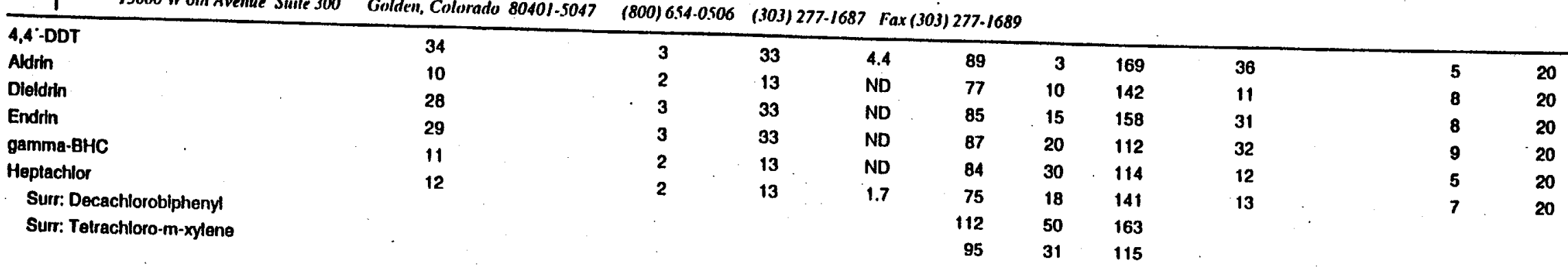

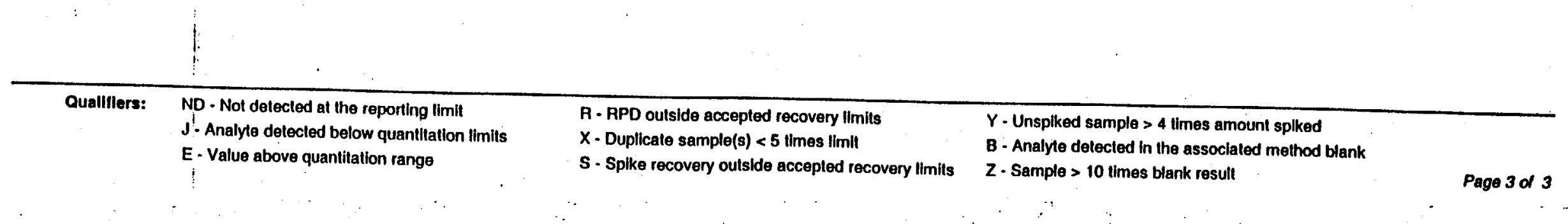




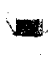


Job Number: 23220

Facllity 10: wrs

Smpling Event: cat 340

COC Number: cau 340-2

Laboratory: BARINGR
Cooler 1D:

Cooler Temp:

Number ol Coolers:

Cooler Units:
Dellvery Order 1D:

$w \mid 761$

SEIR No.: CAU 340-2-O

charge code: $\mathrm{C}(\mathrm{C}) 230 / 7 \mathrm{At}$

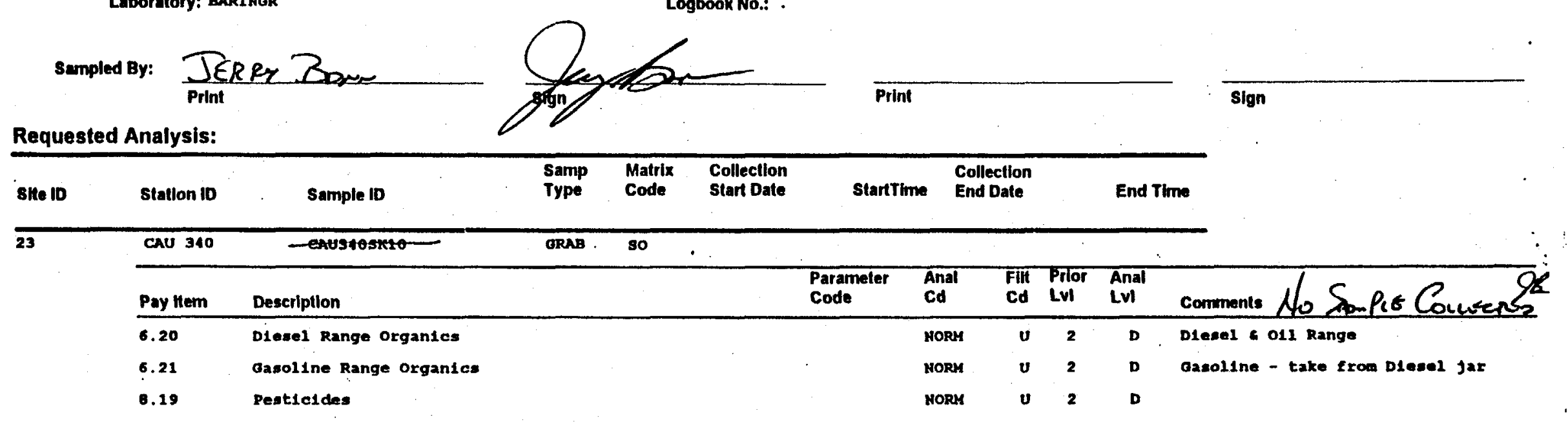

$\begin{array}{lllll}\text { CAU } 340 & \text { CAU340sK11 } & \text { aRAB } & \text { so } & 116698\end{array}$

Pay Item Description

0.19 Pestioldes

0805

Parameter

Anal $1 / 6 / 98$

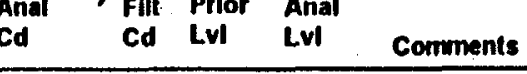

23

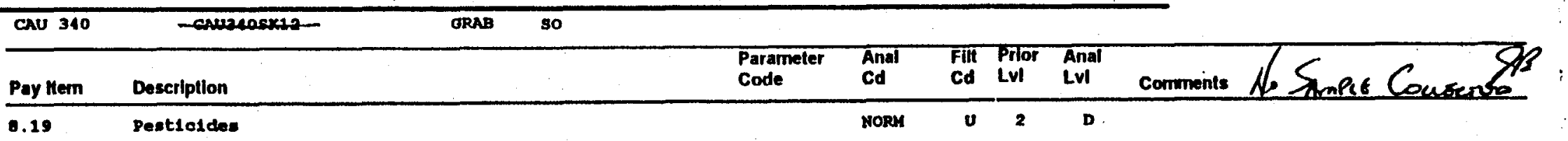

$\cdots$

This package coniorms to the conditions and limitations specilied in 49 CFR 173.421 for excepted radioactive malerial, Himited quantity, n.0.8., UN2910. 
Requested Analysis:

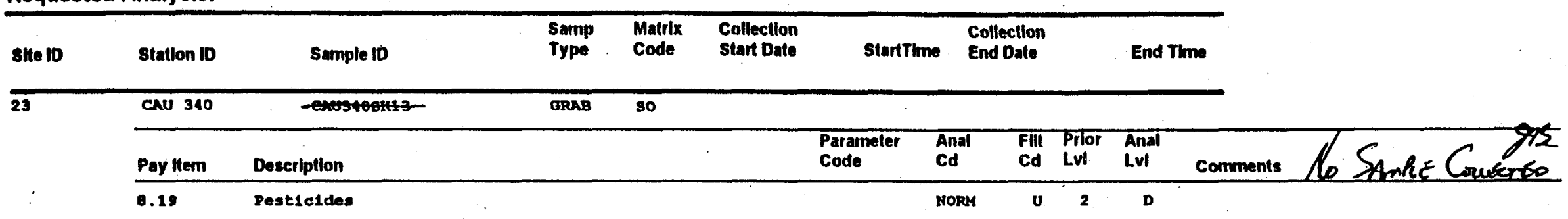

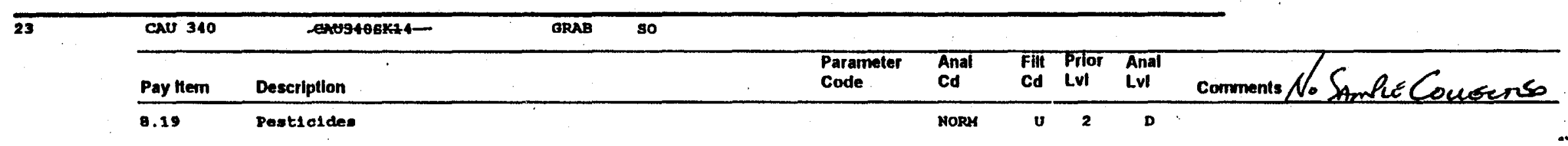

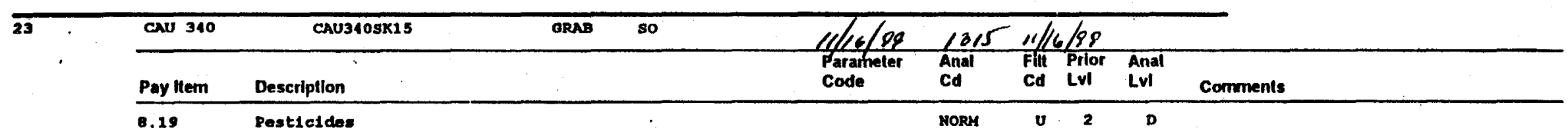

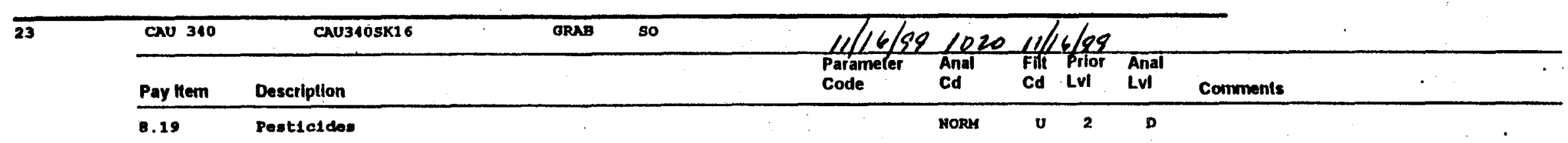




\section{Requested Analysis:}

\begin{tabular}{|c|c|c|c|c|c|c|c|c|c|c|}
\hline SHe ID & station id & Sample ID & $\begin{array}{l}\text { Samp } \\
\text { Type }\end{array}$ & $\begin{array}{l}\text { Matrix } \\
\text { Code }\end{array}$ & $\begin{array}{l}\text { Collection } \\
\text { start Date }\end{array}$ & StertThe & $\begin{array}{l}\text { Collection } \\
\text { End Date }\end{array}$ & & End Thre & \\
\hline \multirow[t]{2}{*}{23} & Cav 340 & CAU340SK17 & ORAB & so & $11 / 16 / 99$ & 1025 & $11 / 16 / 99$ & & & \\
\hline & Pay flem & Description & & & & $\begin{array}{l}\text { Parameter } \\
\text { Code }\end{array}$ & id & $\begin{array}{l}\text { Prior } \\
\text { Lvi }\end{array}$ & $\begin{array}{l}\text { Anal } \\
\text { LvI }\end{array}$ & $\therefore$ \\
\hline
\end{tabular}

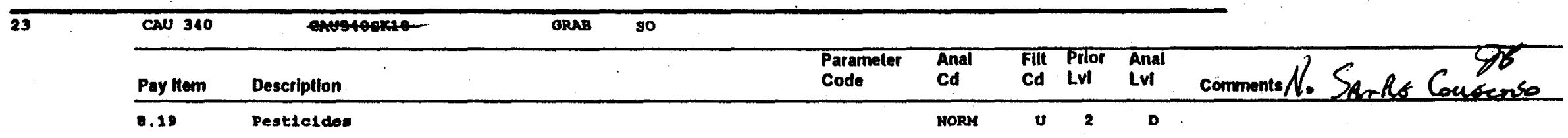

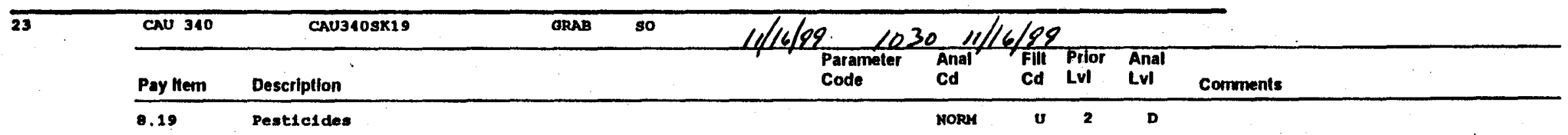

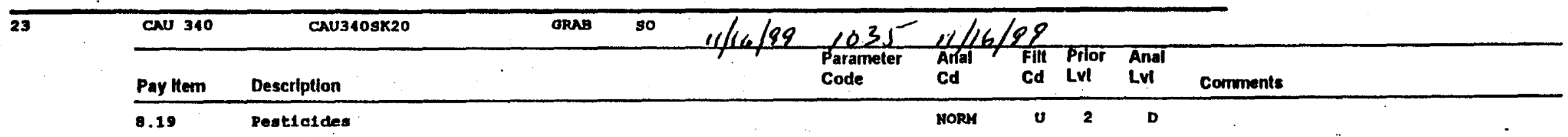


Requested Analysis:

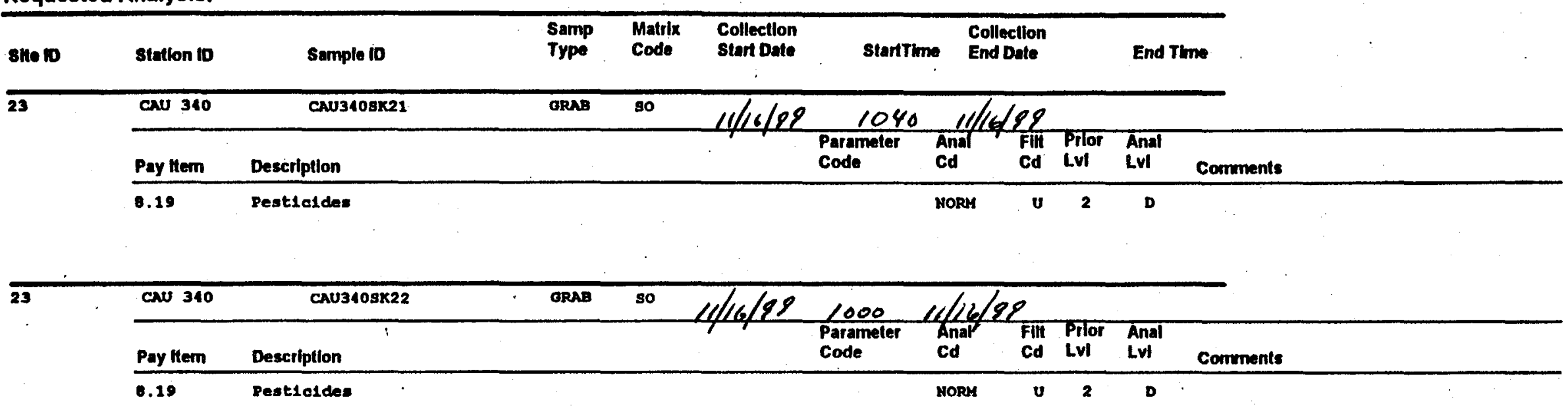

$$
\begin{aligned}
& \text { CAU340atto3 11/10/98 } 084511 / 16 / 99 \\
& \text { Pesticides Norm } u 2 \text {, }
\end{aligned}
$$




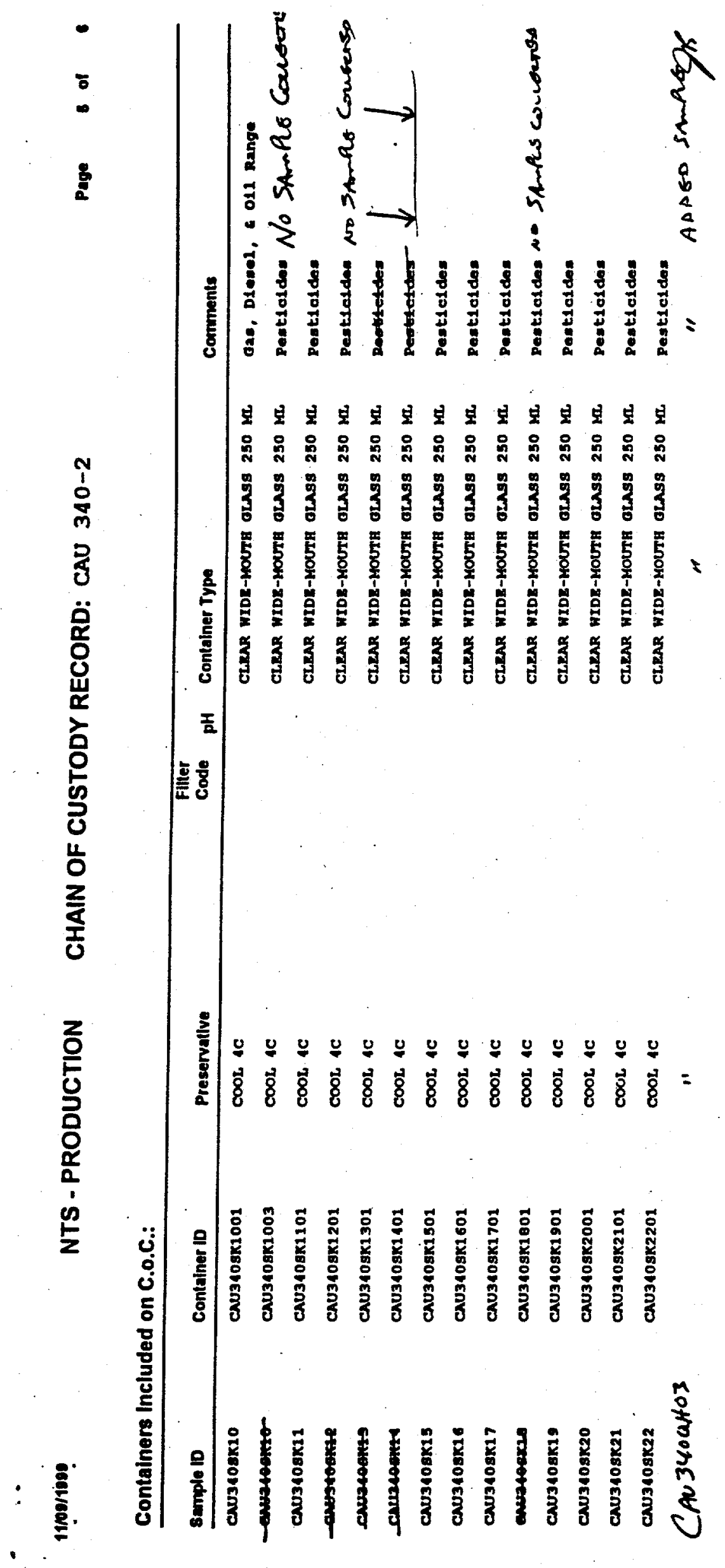


Transfor Information:

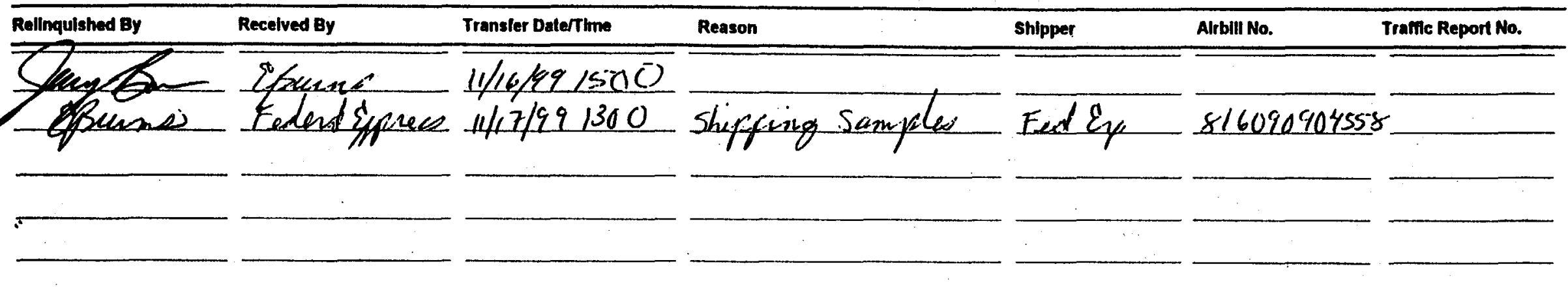

Comments:

Potential Contamination Yes No

Radiological
Chemical


Job Number: 23220

Facillity 10: irs

Sompling Event: cNo 340

coc Number: cas $340-3$

Laboratory: BNRIMOR

\section{Cooler 1D:}

Cooler Temp:

Number of Coolers:

Cooler Units:

Logbook No.:
Dellvery Order ID:

SEIR No.: CAU 340-3-0

charge code: COOOZ $44 A$

sampled By: TERRY Borr

Requested Analysis:

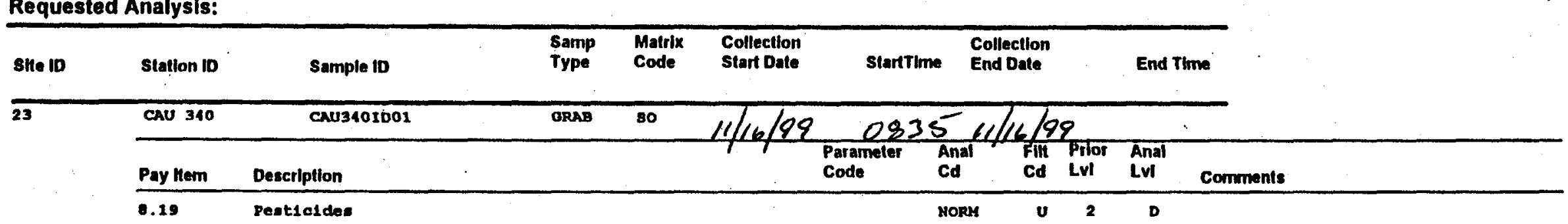

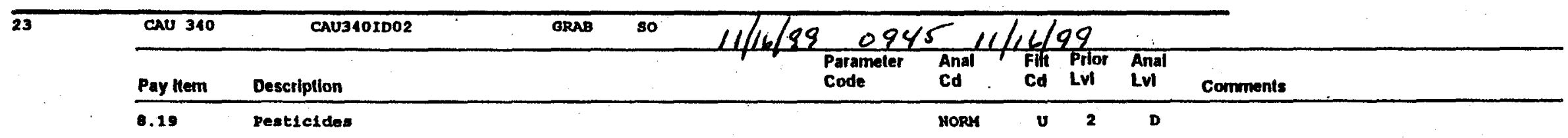

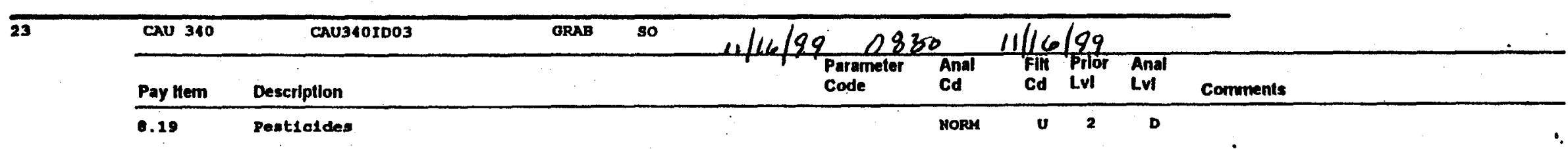


Requested Analysis:

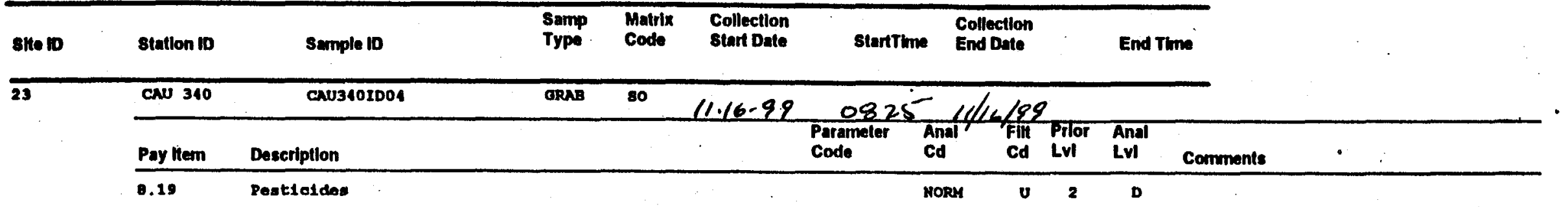

\begin{tabular}{|c|c|c|c|c|c|c|c|c|c|c|}
\hline \multirow[t]{2}{*}{23} & CAU 340 & CAU340IDOS & ORAB & 80 & 11.10 .99 & 0820 & $11 / 16 / 89$ & & & \\
\hline & Pay Kem & Description & & & & $\begin{array}{l}\text { Parameler } \\
\text { Code }\end{array}$ & $\begin{array}{ll}\text { Anal } & \text { Fith } \\
\text { Cd } & \text { Cd }\end{array}$ & $\begin{array}{l}\text { Prlor } \\
\text { LvI }\end{array}$ & $\begin{array}{l}\text { Anal } \\
\text { LvI }\end{array}$ & Comments \\
\hline
\end{tabular}

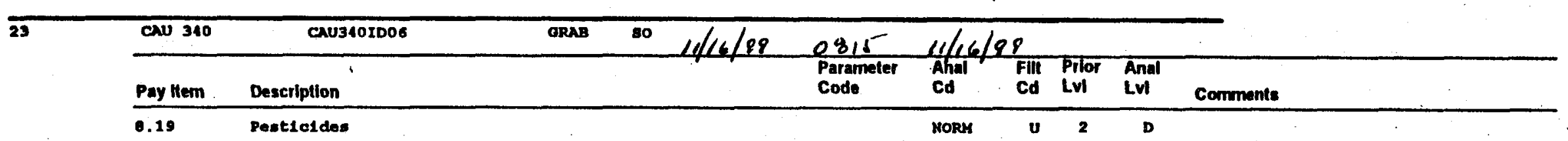

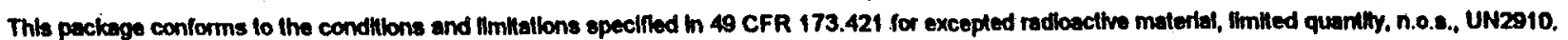




\section{Containers Included on C.o.C.:}

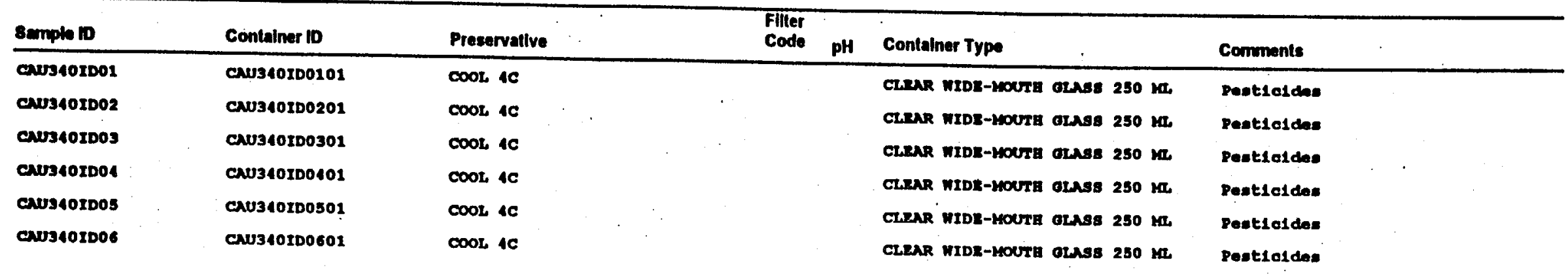




\section{Transfer Information:}

Relinquished By Recelved By Transfer DaterThre Reason shipper Alrbill No. Tramic Report No.

isurims Esterna $\$ 16 / 991500$ ,

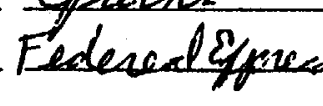
. Shazfing Samples Fed ac 816090904558

\section{Commanis:}

Potential Contamination Yes No.

Radlological
Chemical




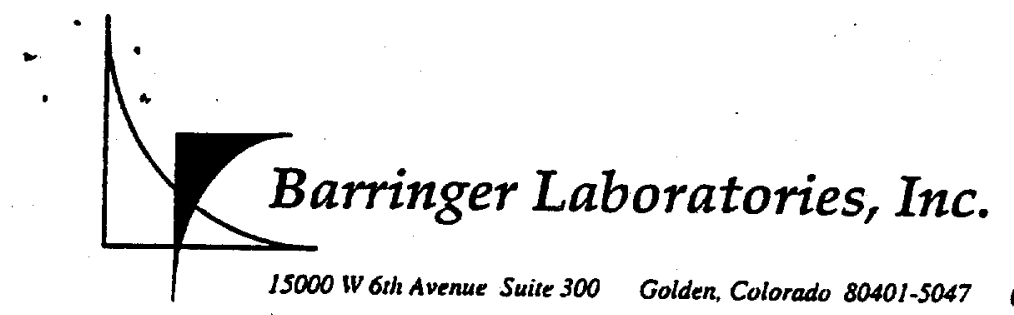

Date: 11-Feb-00

Ted Redding

Bechtel Nevada Corp.

P.O. Box 98521

MS NTS 273

Las Vegas, NV 89193-8521

Phone: 1-702-295-7220

Fax: 1-702-295-4773

Work Order: 9911170

Project: V725

Dear Ted Redding,

Barringer Laboratories received 5 samples on $11 / 19 / 99$ for the analyses presented in the following report.

There were no problems with the analyses and all data for associated QC met EPA or laboratory specifications except where noted in the Case Narrative.

If you have any questions regarding these test results, please feel free to call.
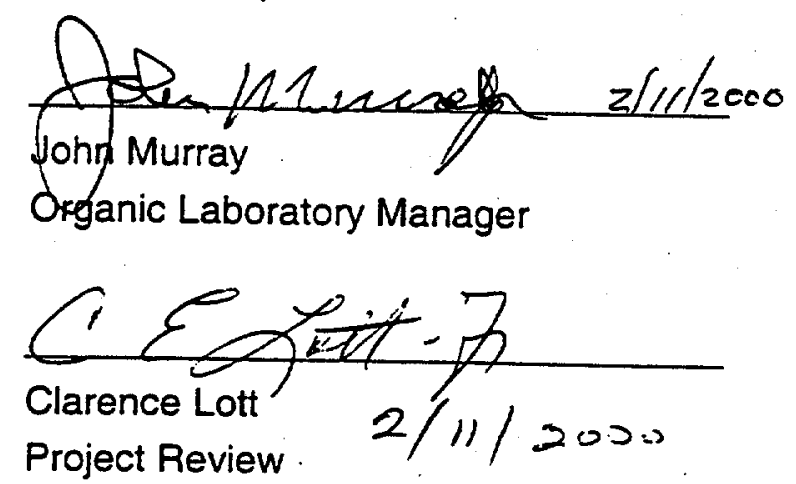
Date: 14-Feb-00

\section{Barringer Laboratories, Inc.}

J5000 W Gth Avenue Suite 300 Golden. Colorado 80401-5047 $\quad$ (800)654-0506 (303) 277-1687 Fax (303) 277.1689

\begin{tabular}{llll}
\hline Client: & Bechtel Nevada Corp. & \\
Project: & V725 & CASE NARRATIVE \\
Work Order: & 9911170 &
\end{tabular}

All reported values in this report have been rounded to the correct number of significant figures. All calculations have been performed before applying significant figures, therefore, not all calculations may be reproducible with the results printed in this report.

Analytical Comments for METHOD SW846 8015BGRO, SAMPLE MBLK: The surrogate exceeded the set QC limit for the method blank which was non-detect for gasoline. Because the surrogate was high, we believe gasoline would be detected if present and therefore consider the data to be valid as reported.

Analytical Comments for METHOD SW846 8081A, SAMPLE 9911170-01AMS: The high concentration of 4,4-DDT in the sample caused the percent recovery and RPD to fail their set QC limits.

Analytical Comments for METHOD SW846 8081A, SAMPLE 9911170-01A: The result for gamma-Chlordane is being reported from the second column because of an interfering peak on the primary column.

Analytical Comments for METHOD SW846 8081A, SAMPLE 9911170-03A: The result for gamma-Chiordane is being reported from the second column because of an interfering peak on the primary column.

Analytical Comments for METHOD SW846 8015BDRO: The sample was originally extracted on 11/29/1999 but when analyzed the QC failed for the LCS/LCSD and MS/MSD. They were re-extracted on 12/3/1999, which was beyond the recommended holding time for soils. The OC passed on the re-extracted and re-anlayzed samples. Both analysis had results below the reporting limit of $10 \mathrm{mg} / \mathrm{kg}$ with a $30 \%$ difference in the analytical result, the first analysis being $4.6 \mathrm{mg} / \mathrm{kg}$ and the second being $6.3 \mathrm{mg} / \mathrm{kg}$.

This report has been amended from the previous report dated 12/9/99 to remove the 10X dilution result for alpha-chlordane associated with sample $9911170-03 \mathrm{~A}$ as requested by Ted Redding on 2/10/2000. The extract was analyzed twice once undiluted and once at a 10X dilution with both analyses yielding a result of $49 \mathrm{ug} / \mathrm{Kg}$.

The sample ID was corrected based on the c.0.c. for sample 9911170-02A at the request of Ted Redding on 2/10/2000 from CAU340S12 to CAU340SK12. 


\section{Barringer Laboratories, Inc.}

15000 W Gih Avenue Suite 300 Golden, Colorado 80401-5047.

(800) $654-0506$

(303) 277.1687 Fax (303) 277-1689

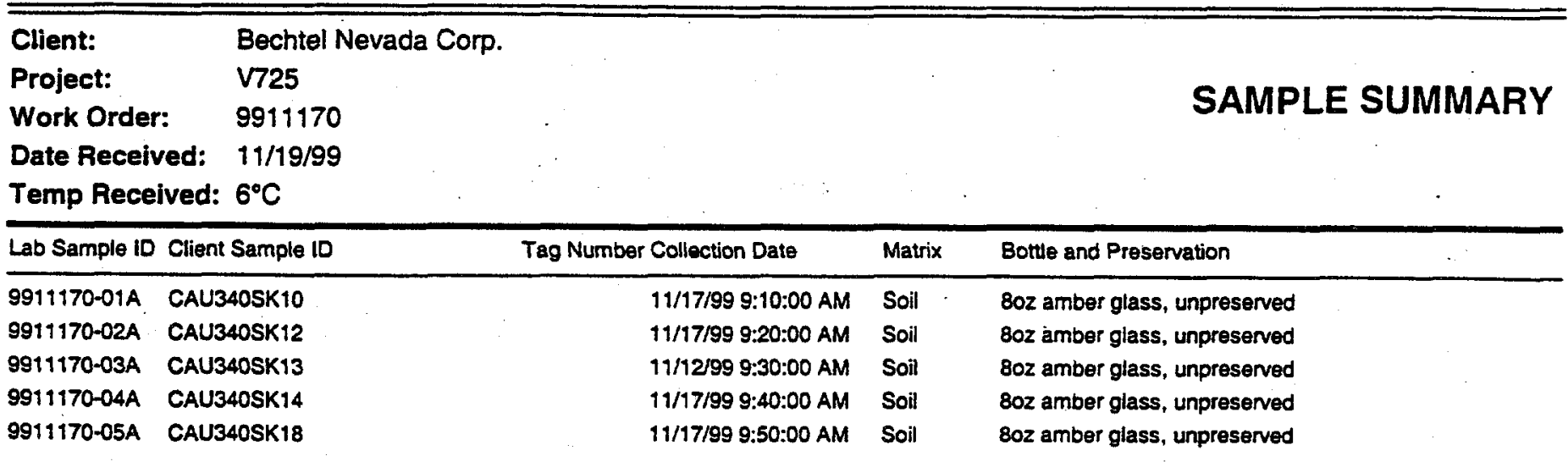


Date: 14-Fob-00 Barringer Laboratories, Inc. CLIENT SAMPLE REPORT

15000 W Gill Avemue Suite 300 Golden. Colorado 80401.5047 (800)654-0.506 (303) 277.1687 Fax (303) 277.1689

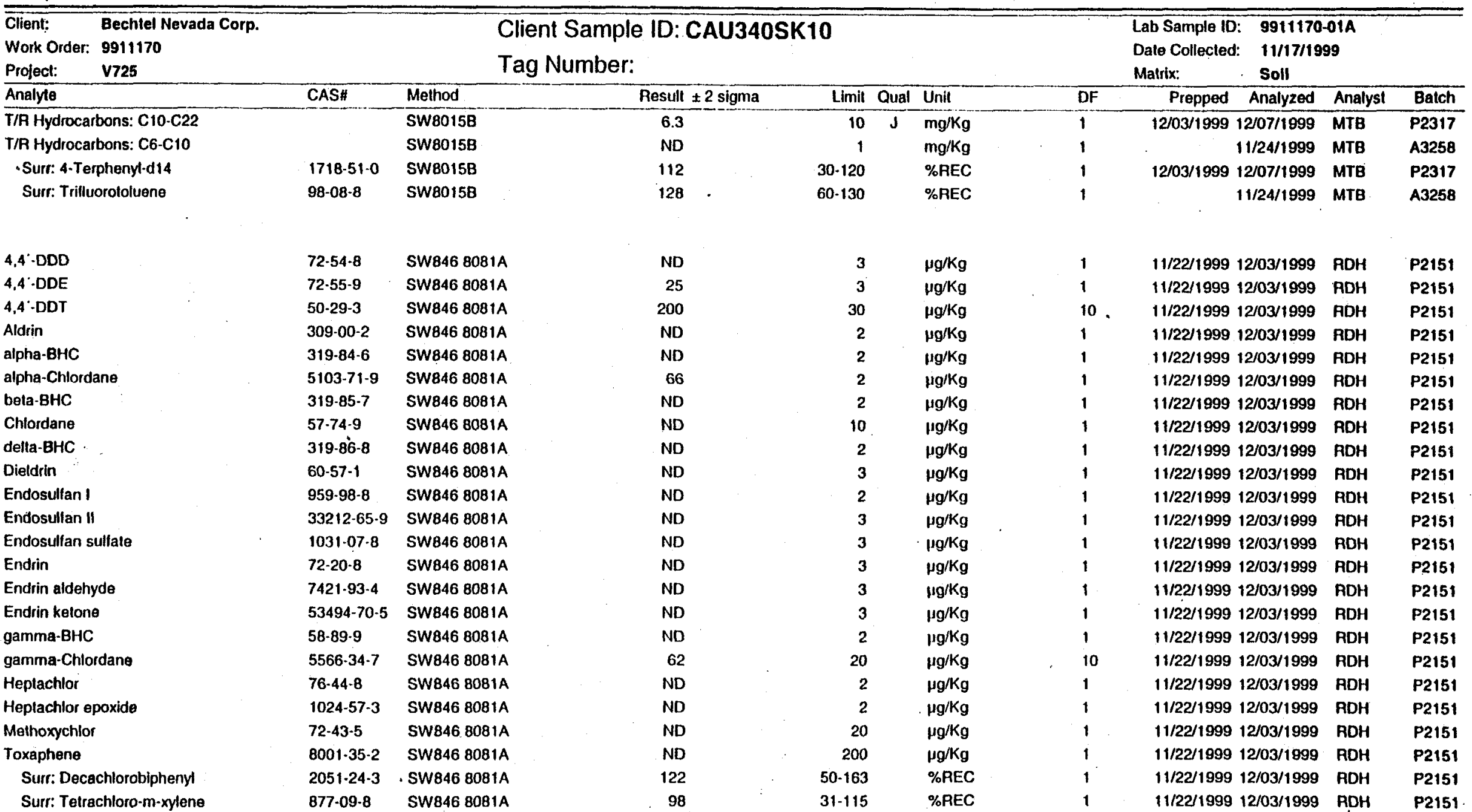

$\begin{array}{ll}\text { Qualiliers: } & \text { ND - Not delected at the reporting limit } \\ & \text { J - Analyte detected below quantilation limils } \\ & E \text { - Value above quantilation range }\end{array}$
R - RPD outside accepted recovery limits

$X$ - Duplicale sample(s) $<5$ limes limil

$S$ - Spike recovery outside accepled recovery limits
$Y$. Unspiked sample $>4$ times amount spiked

$B$ - Analyle delecled in lihe associaled mellod blank

- Sample $>10$ limes blank resull 
Date: 14-Fob-00 Barringer Laboratories, Inc.

CLIENT SAMPLE REPORT

15000 W 6ilh Avemue Suire 300 Golden, Colorado 80401-5047 (800)6.54.0506 (303) 277-1687 Fax (303) 277.1689

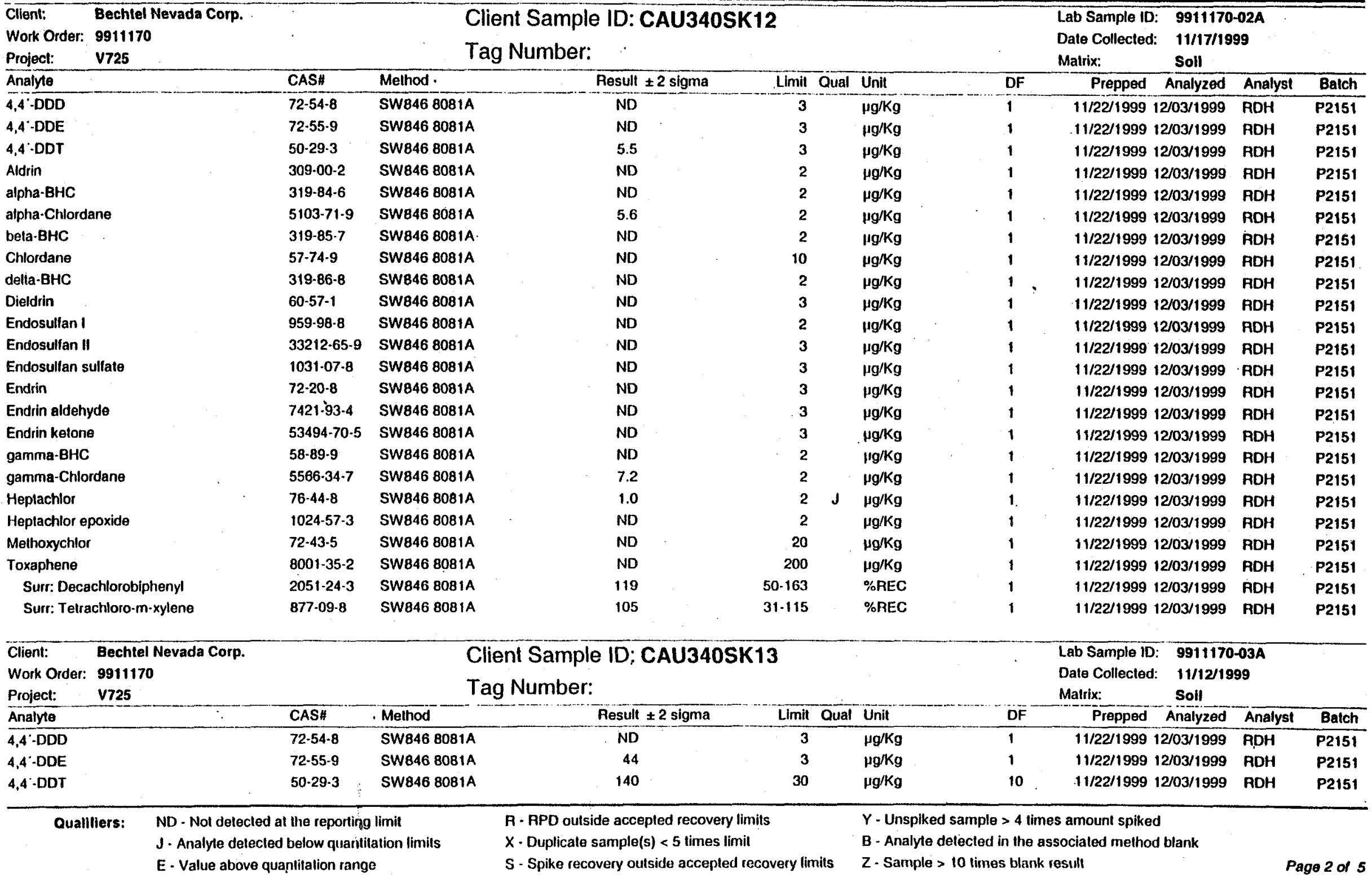


Uate: 14-Feb-00 Barringer Laboratories, Inc.

\section{CLIENT SAMPLE REPORT}

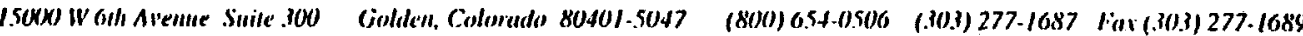

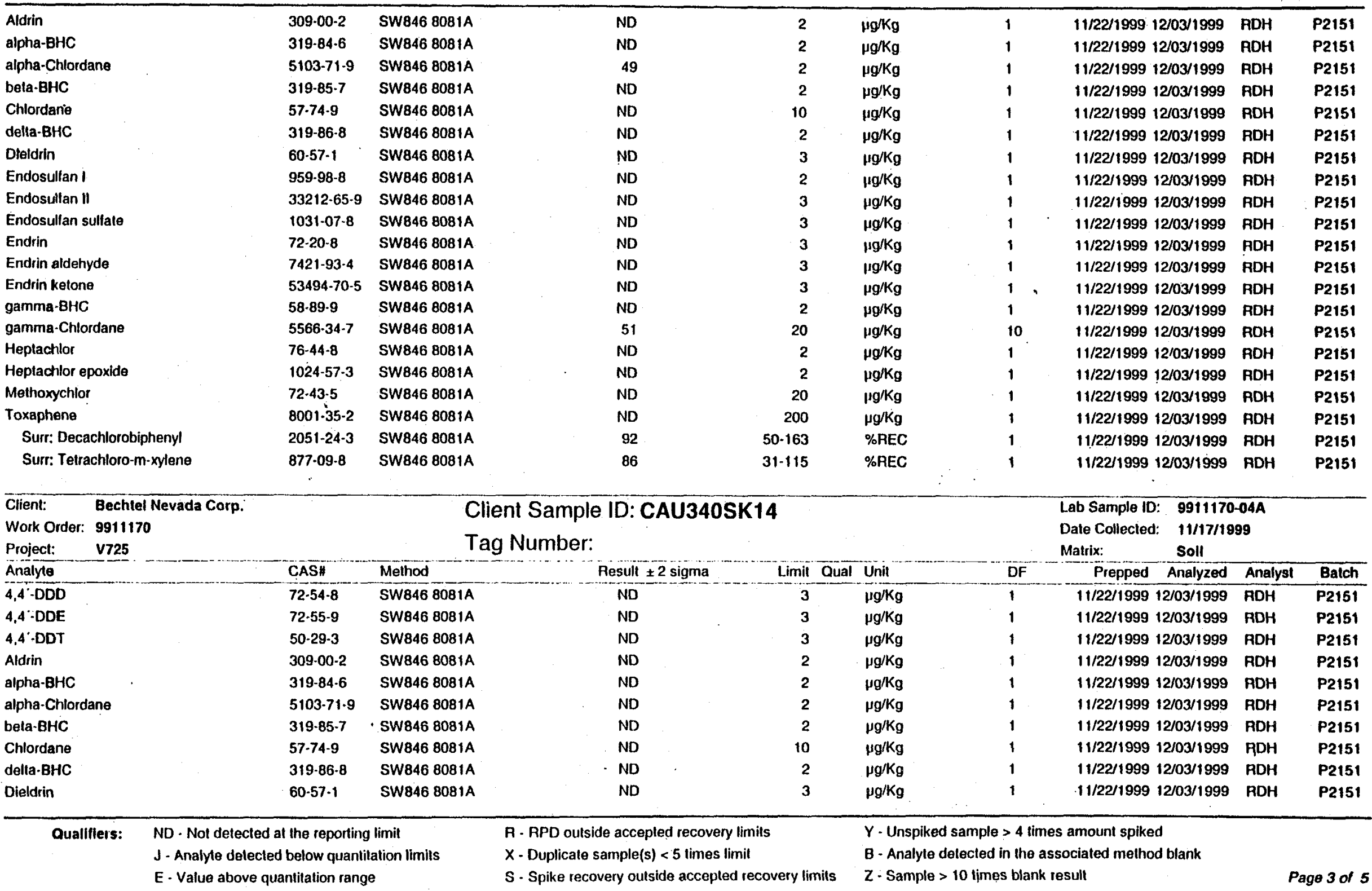


Date: 14-Feb-00 Barringer Laboratories, Inc.

CLIENT SAMPLE REPORT

15000 W6th Averure Suite 300 Golden, Colorado 80401-5047 (800)6.54.0506 (30.3) 277-1687 Fax (30.3) 277.1689

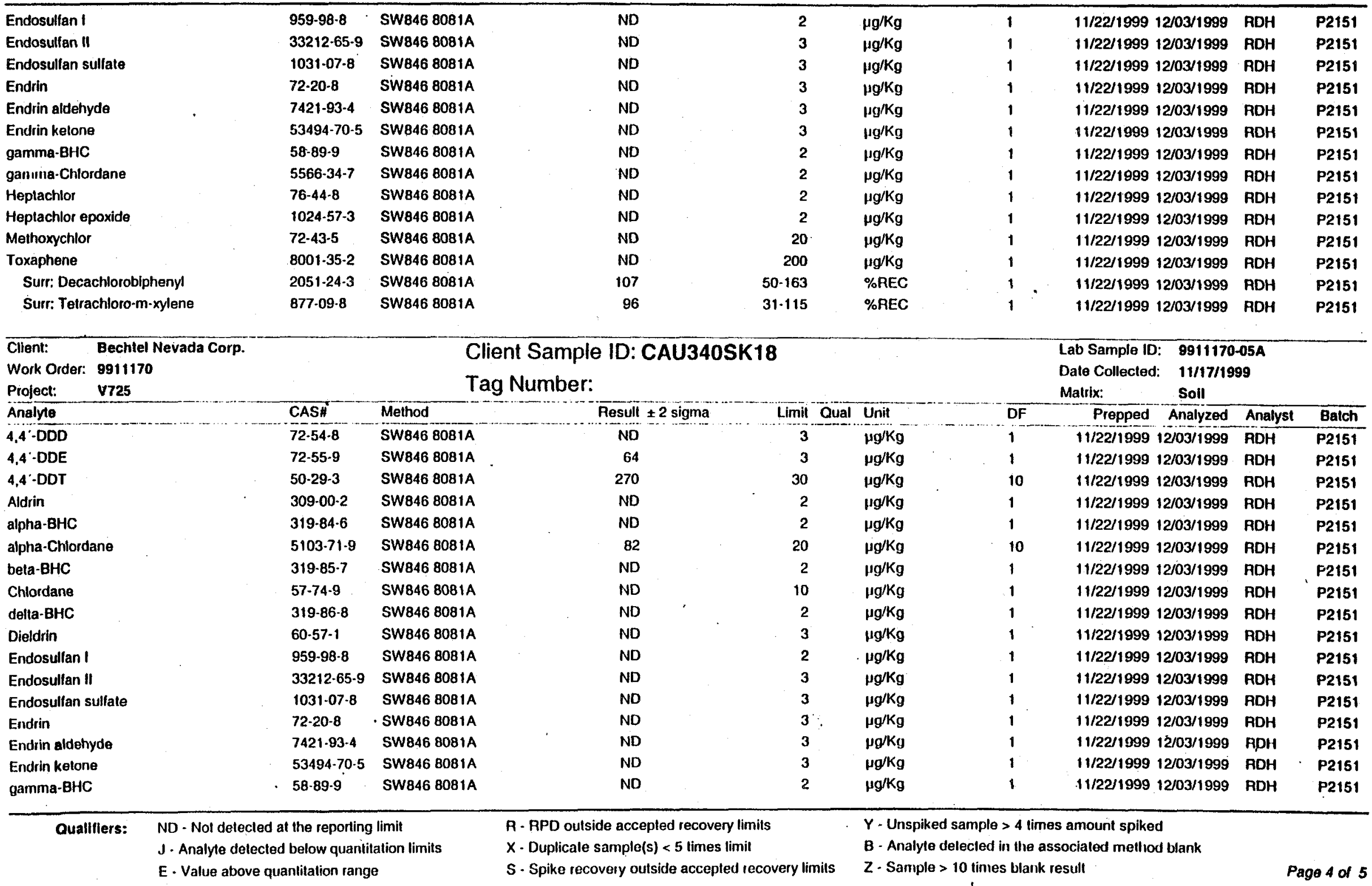

E. Value above quantitalion range

$S$ - Spike recovery outside accepted recovery limits $\quad Z$ - Sample $>10$ times blank result 
15000 w Gilh Aventue Suile 300

Golden, Colorado 80401.5047 (800) 654-0506 (303) 277.1687 Fax (30.3) 277.1689

gamma-Chlordane

Heptachlor

5566.34.7 SWB46 8081A

76.44-8 SW846 B081A

ND

1024-57-3 SW846 B081A

Melhoxychlor

Toxaphene

Surr. Decachlorobiphenyl

Surr: Telrachloro-m-xylene

72-43-5 SW846 B081A

NO

8001-35-2 SW846 8081A

ND

ND

2051-24-3 SW846 8081A

108

877.09-8 SW846 8081A

94

CLIENT SAMPLE REPORT

$\mu g / \mathrm{Kg}$

$\mu \mathrm{g} / \mathrm{Kg}$

$\mu g / \mathrm{Kg}$

$\mu g / K g$

$\mu g / K g$

\%REC

$\%$ REC
$11 / 22 / 1999$ 12/03/1999 $\mathrm{RDH}$

$11 / 22 / 1999$ 12/03/1999 RDH

$11 / 22 / 1999$ 12/03/1999 ROH

$11 / 22 / 1999$ 12/03/1999 RDH

11/22/1999 12/03/1999 RDH

$11 / 22 / 1999$ 12/03/1999 RDH

11/22/1999 12/03/1999 RDH
P2151

P2151

P2151

P2151

P2151

P2151

P2151 $\begin{array}{ll}\text { Quallilers: } & \text { ND - Not delected at the reporting limil } \\ & \text { J - Analyle detected below quantilation limits } \\ & \text { E - Value above quantilation range }\end{array}$
R - RPD outside accepted recovery limits

$X$ - Duplicate sample(s) $<5$ times limil

S - Spike recovery oulside accepted recovery limils $\gamma$ - Unspiked sample $>4$ times amount spiked

B - Analyte delecled in the associated method blank

$Z$ - Sample $>10$ limes blank resull 


\section{THIS PAGE INTENTIONALLY LEFT BLANK}


15000 W6 Glt Averme Suite 300. Golden, Colorade 80401.5047

\section{BATCH QC SUMMARY REPORT}

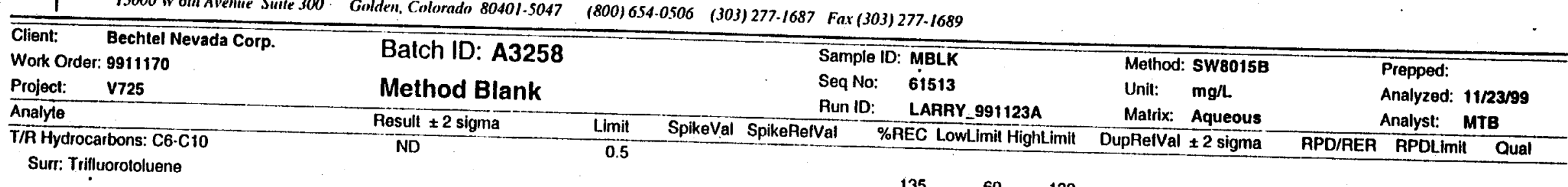
Surr: Trilluorotoluene

$$
135 \quad 60 \quad 130
$$

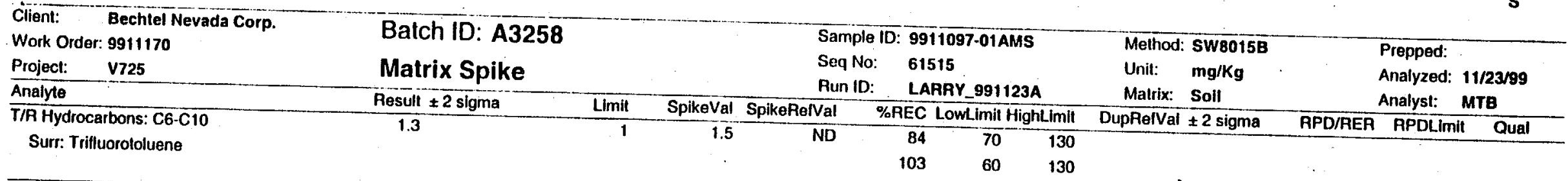

\begin{tabular}{|c|c|c|c|c|c|c|c|c|c|c|c|c|c|}
\hline$\frac{\text { Analyte }}{\text { T/R Hydroc }}$ & & $\frac{\text { Resull } \pm 2 \text { sigma }}{1.2}$ & Limit & SpikeVal & SpikeRerval & \%REC I & LowLimil H & ghimil & DupRefVal & \pm 2 sigma & APD/REA & RPDLimit & Qual \\
\hline $\begin{array}{l}\text { S/R Hydroc } \\
\text { Surr: Trif }\end{array}$ & $\begin{array}{l}\text { arbons: C6-C10 } \\
\text { luorotoluene }\end{array}$ & 1.2 & 1 & 1.5 & ND & $\begin{array}{r}78 \\
104\end{array}$ & $\begin{array}{l}70 \\
60\end{array}$ & $\begin{array}{l}130 \\
130\end{array}$ & 1.3 & & 7 & 20 & \\
\hline
\end{tabular}

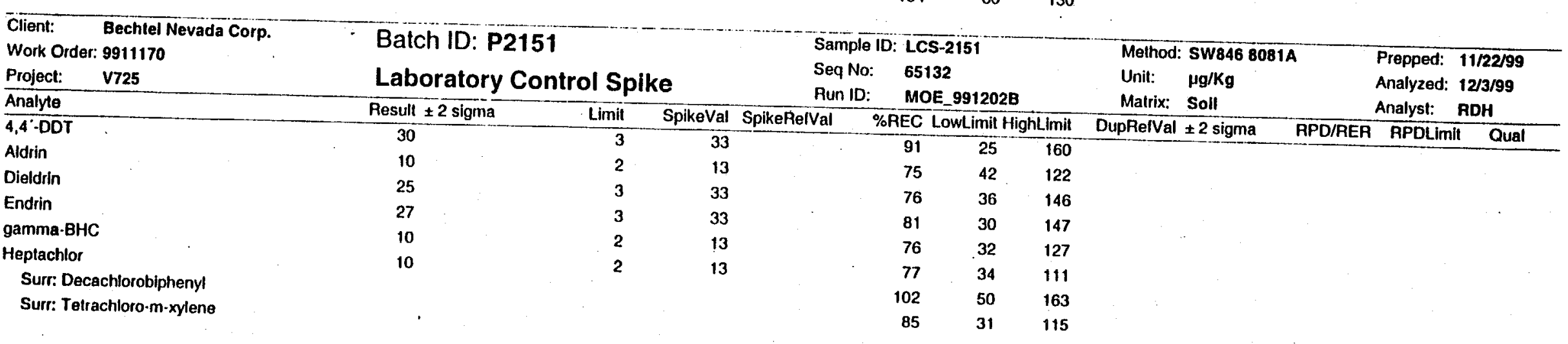

\begin{tabular}{|c|c|c|c|}
\hline Qualitiers: & $\begin{array}{l}\text { ND - Nol delected at the reporting limil } \\
\text { J - Analyte delecled below quantitation limils } \\
\text { E - Value above quantitation range }\end{array}$ & $\begin{array}{l}\text { A - RPD outside accepted recovery limits } \\
\text { X-Duplicale sample(s) }<5 \text { times limil } \\
\text { S - Spike recovery oulside accepted recovery limils }\end{array}$ & $\begin{array}{l}Y \text { - Unspiked sample }>4 \text { limes amount spiked } \\
\text { B - Analyte delected in the assacialed method blank } \\
Z \text { - Sample }>10 \text { times blank result }\end{array}$ \\
\hline
\end{tabular}



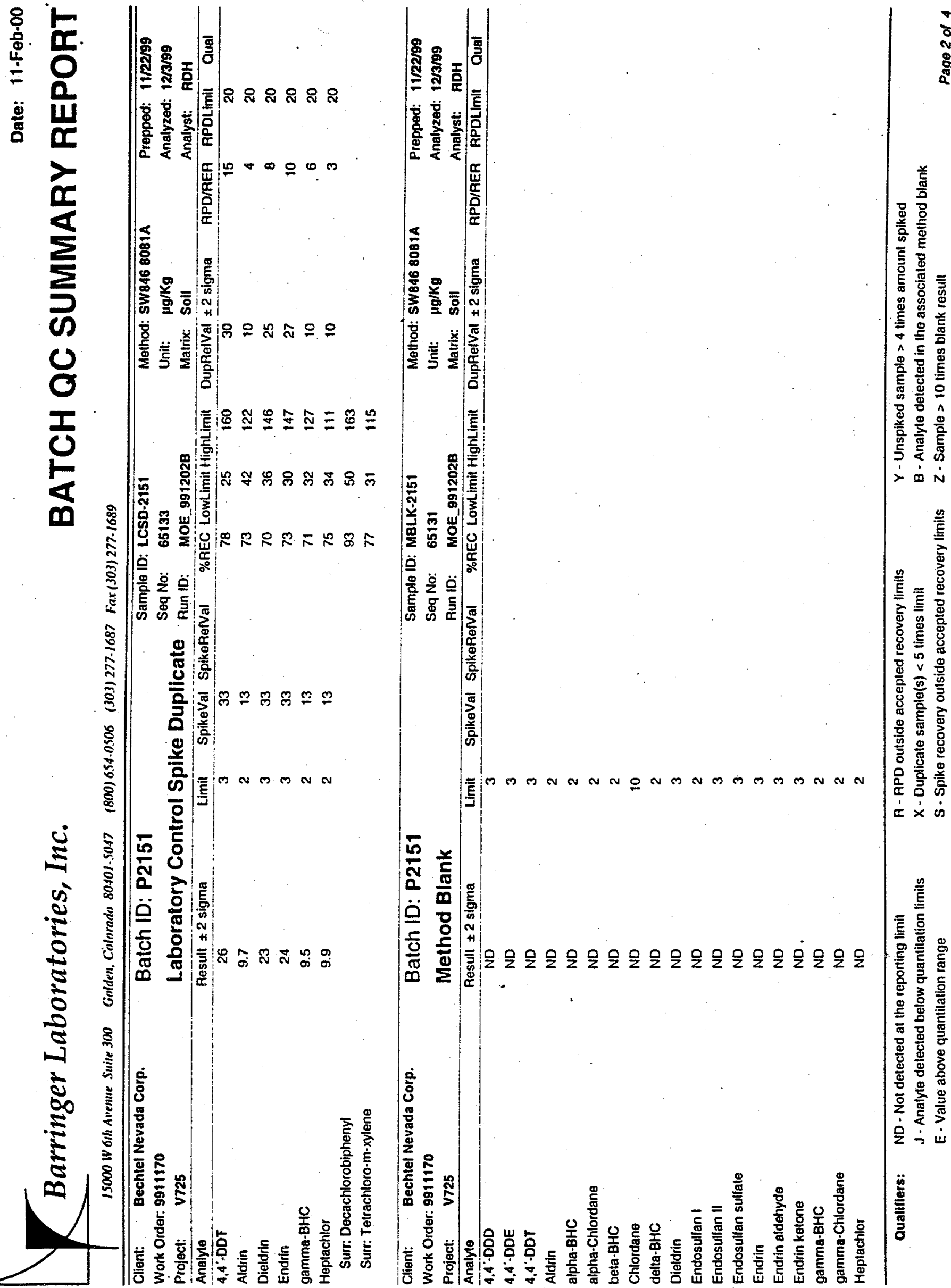
Date: 11-Feb-00 Barringer Laboratories, Inc.

BATCH QC SUMMARY REPORT

15000 W 6ih Avemue Suite 300 Gulden, Colorado 80401-5047 (800) 654-0506 (30.3) 277-1687 Fax (30.3) 277-1689

$\begin{array}{llr}\text { Heptachlor epoxide } & \text { ND } \\ \text { Methoxychlor } & \text { ND } & 20\end{array}$

Toxaphen

ND

20

Surr: Decachlorobiphenyl

200

Surr: Tetrachloro-m-xylene

$116 \quad 50 \quad 163$

\begin{tabular}{lcc}
\hline Client: Bechtel Nevada Corp. & Balch ID: P2151 \\
Work Order: 9911170 & Matrix Spike \\
Project: V725 & Result \pm 2 sigma & Limit \\
\hline Analyte & 190 & 3 \\
\hline $4,4 \cdot$ ODI & 12 & 2 \\
Aldrin & 26 & 3 \\
Dieldrin & 28 & 3 \\
Endrin & 10 & 2 \\
gamma-BHC & 12 & 2 \\
Heptachlor & &
\end{tabular}

Surr: Tetrachloro-m-xylen

$\begin{array}{rrr}90 & 31 & 115\end{array}$

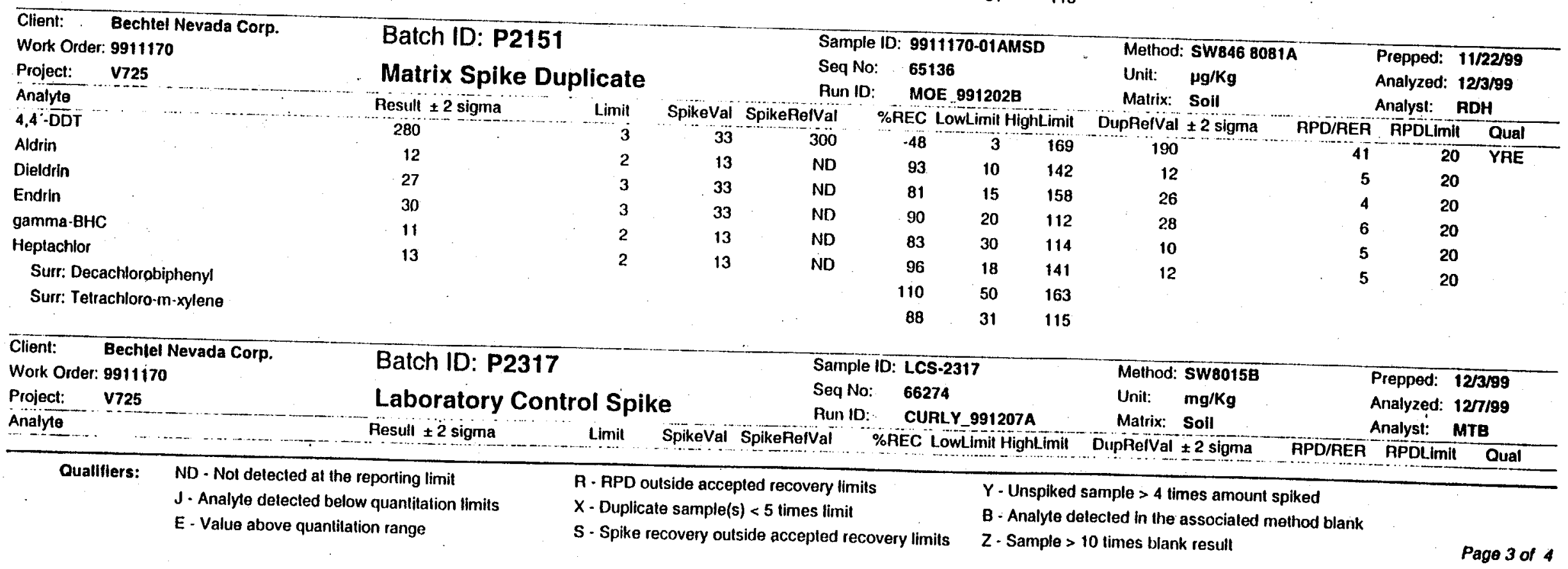


Date: 11-Feb-00 Barringer Laboratories, Inc.

BATCH QC SUMMARY REPORT

$\begin{array}{lllll}15000 \text { W Gth Avenue Suive } 300 \quad \text { Golden, Colurado 80401-5047 } & \text { (800) 6.54-0.506 (303) 277-1687 Fax (.30.3) 277.1689 }\end{array}$

\begin{tabular}{ccccccc}
\hline T/R Hydrocarbons: C10-C22 & 25 & 10 & 33 & 76 & 30 & 120 \\
Surr: 4-Terphenyl-d14 & & & & 81 & 30 & 120
\end{tabular}

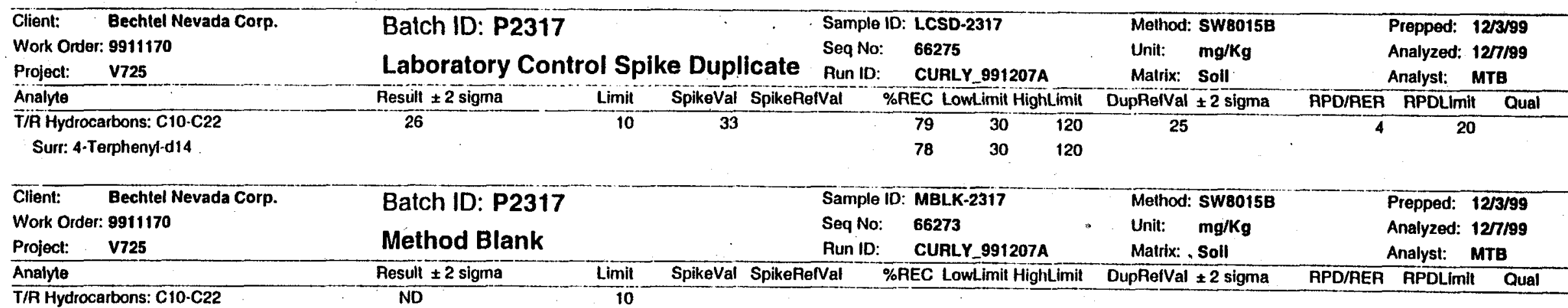

$80 \quad 30 \quad 120$

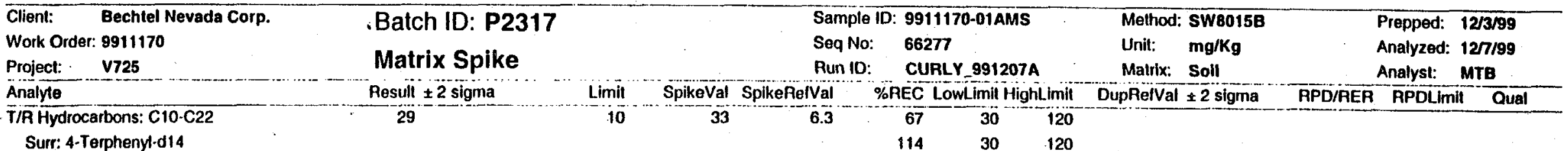

Surr: 4-Terphenyl-d14

$\begin{array}{lll}114 & 30 & 120\end{array}$

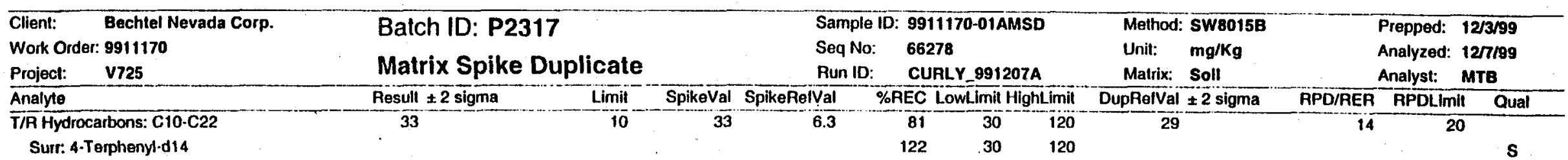

Qualliters: ND - Nol detected at the reporting limit

J. Analyte detecled below quantitation limits

E. Value above quantitation range
$R$ - RPD outside accepted recovery limits

$X$ - Duplicate sample(s) $<5$ limes limit

S - Spike recovery outside accepted recovery limits
$Y$ - Unspiked sample $>4$ times amount spiked

$B$ - Analyte delected in the associaled method blank

Z - Sample $>10$ times blank result 
Job Number: 23220

Cooler 1D:

Facllity ID: NTS

Sampling Event: CaU 340

COC Number: CNU 340-5

Laboratory: BARIYOR
Cooler Temp:

Number of Coolers:
Dellvery Order 1D:

SEIR NO: CAU 340-2-0

Charge Code: Cooo24M
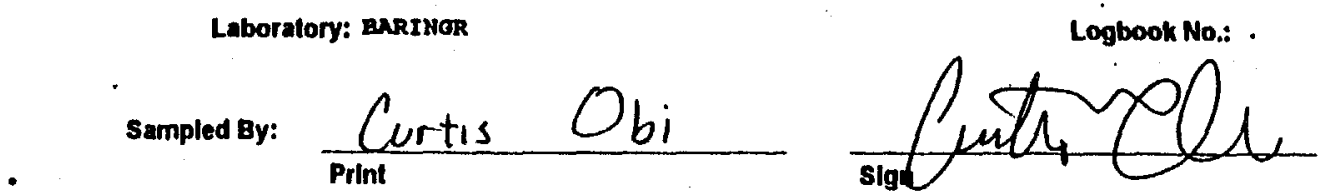

Print

slgn

Requested Analysis:

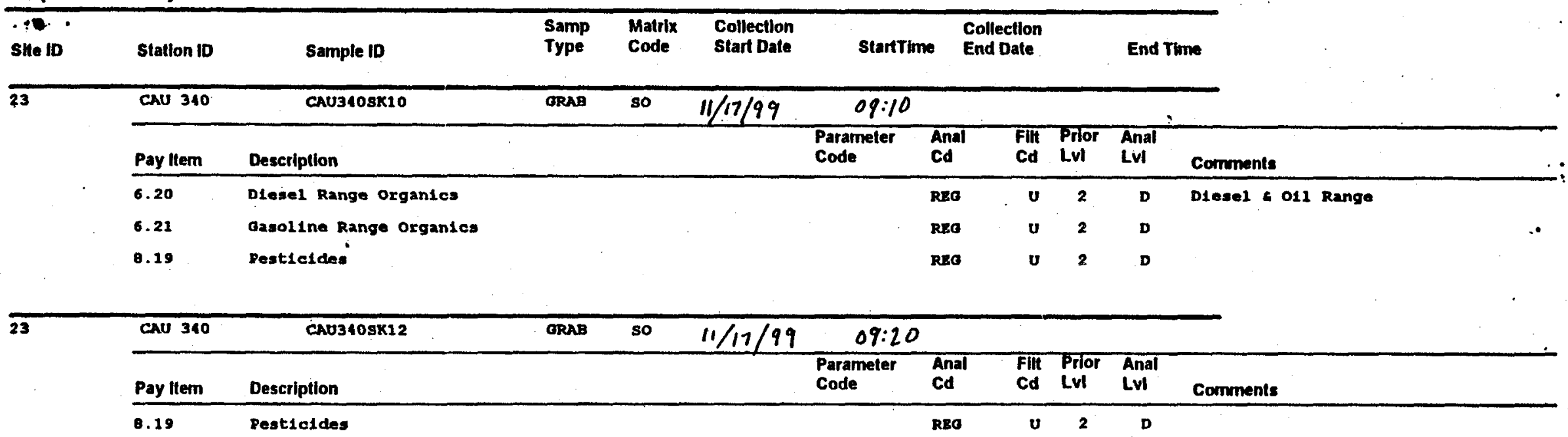

\section{3}

\begin{tabular}{llcccc} 
CAU 340 & CAU340SK13 & ORAB & so & $11 / 12 / 99$ & $09: 30$ \\
\hline Pay hem & Description & & $\begin{array}{l}\text { Parameter } \\
\text { Code }\end{array}$
\end{tabular}

Pay Hern Description

Code

$\begin{array}{lll}\begin{array}{l}\text { Anal } \\ \text { Cd }\end{array} & \begin{array}{l}\text { Fith Prior Anal } \\ \text { Cd }\end{array} & \begin{array}{l}\text { Avl } \\ \text { LvI }\end{array} \text { Comments }\end{array}$

This package conforms to the conditions and limitations specified in 49 CFR 173.421 for excepted radioactive material, limited quanlity, n.0.8., UN2910. 
Requested Analysis:

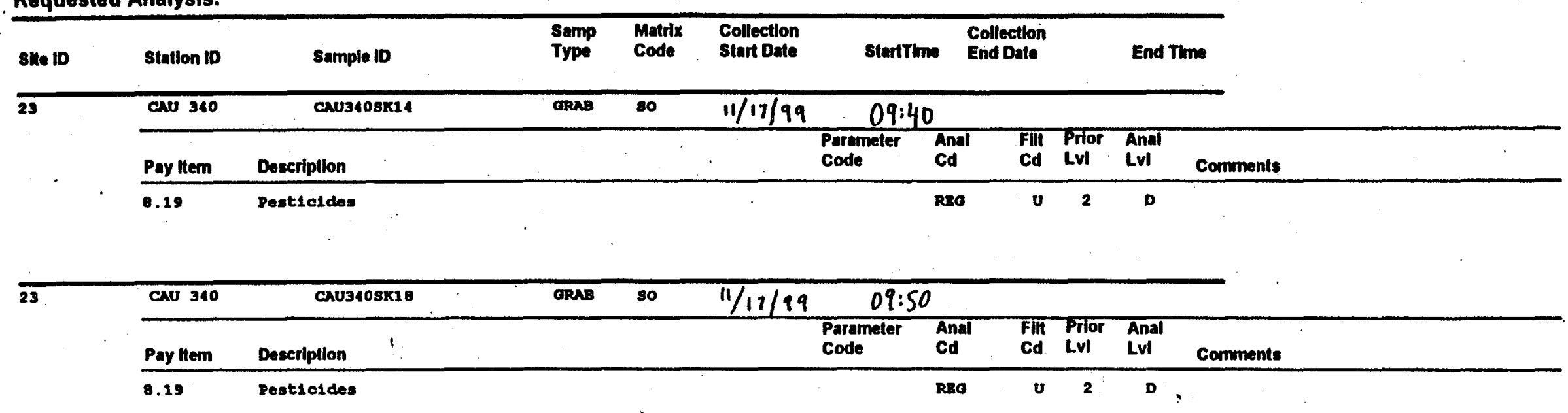


Containers Included on C.o.C.:

\begin{tabular}{|c|c|c|c|c|c|c|}
\hline 8 enple to & Container to & Preservatlve & $\begin{array}{l}\text { Filter } \\
\text { Code }\end{array}$ & pH & Container Type & Cormments \\
\hline 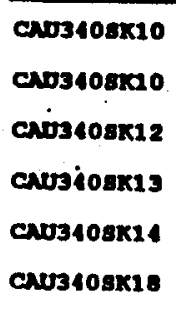 & 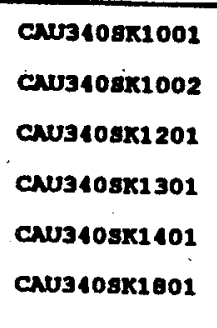 & 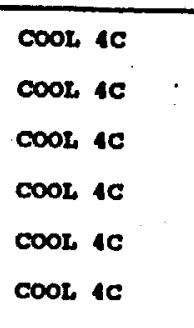 & & & 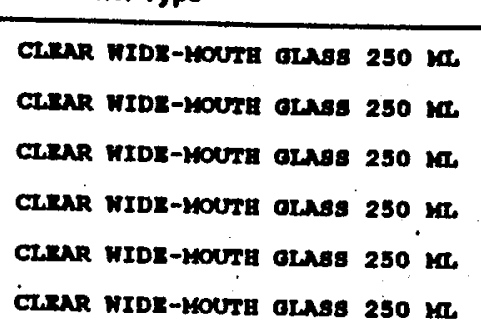 & $\begin{array}{l}\text { Cas, Dlesel, a } 011 \text { Range } \\
\text { pentioldes }\end{array}$ \\
\hline
\end{tabular}


Transfer Informatlon:

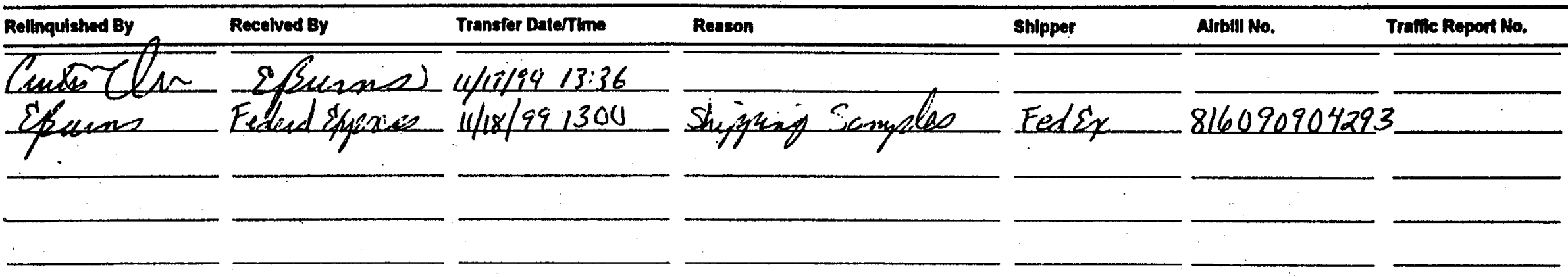

Comments:

Potential Contamination Yes No

$\begin{array}{r}\text { Radiological } \\ \text { Chemical }\end{array}-$




\section{Barringer Laboratories, Inc.}

Date: $17-J a n-00$

$15000 \mathrm{~W}$ 6th Avenue Suite 300

Golden, Colorado 80401-5047

(800)654-0506 (303) 277-J687 Fax (303) 277-1689

Ted Redding

Bechtel Nevada Corp.

P.O. Box 98521

MS NTS 273

Las Vegas, NV 89193-8521

Phone: 1-702-295-7220

Fax: $1-702-295-4773$

Work Order: 0001029

Project: 23220

Dear Ted Redding,

Barringer Laboratories received 6 samples on $01 / 06 / 00$ for the analyses presented in the following report.

There were no problems with the analyses and all data for associated QC met EPA or laboratory specifications except where noted in the Case Narrative.

If you have any questions regarding these test results, please feel free to call.
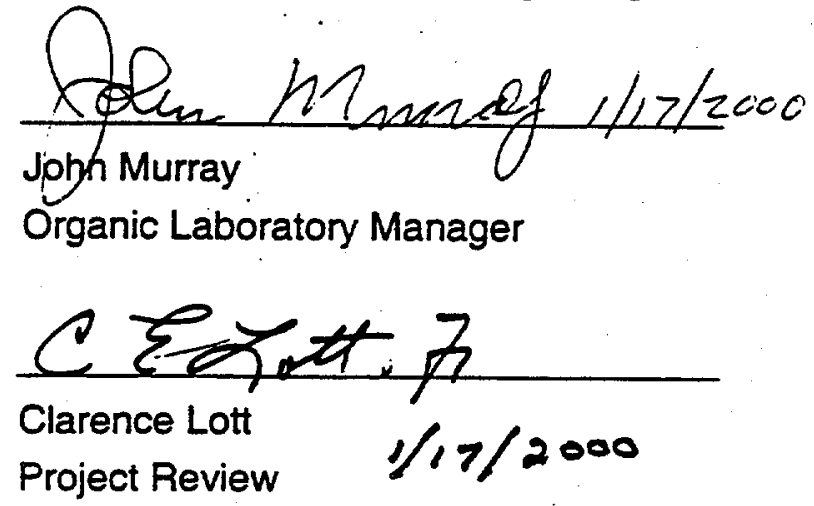
THIS PAGE INTENTIONALLY LEFT BLANK

is 
Date: 17-Jan-00

\section{Barringer Laboratories, Inc.}

15000 W 6th Avenue Suite 300 Golden. Colorado 80401-5047 (800) 654-0506 (303) 277-1687 Fax (303) 277-1689

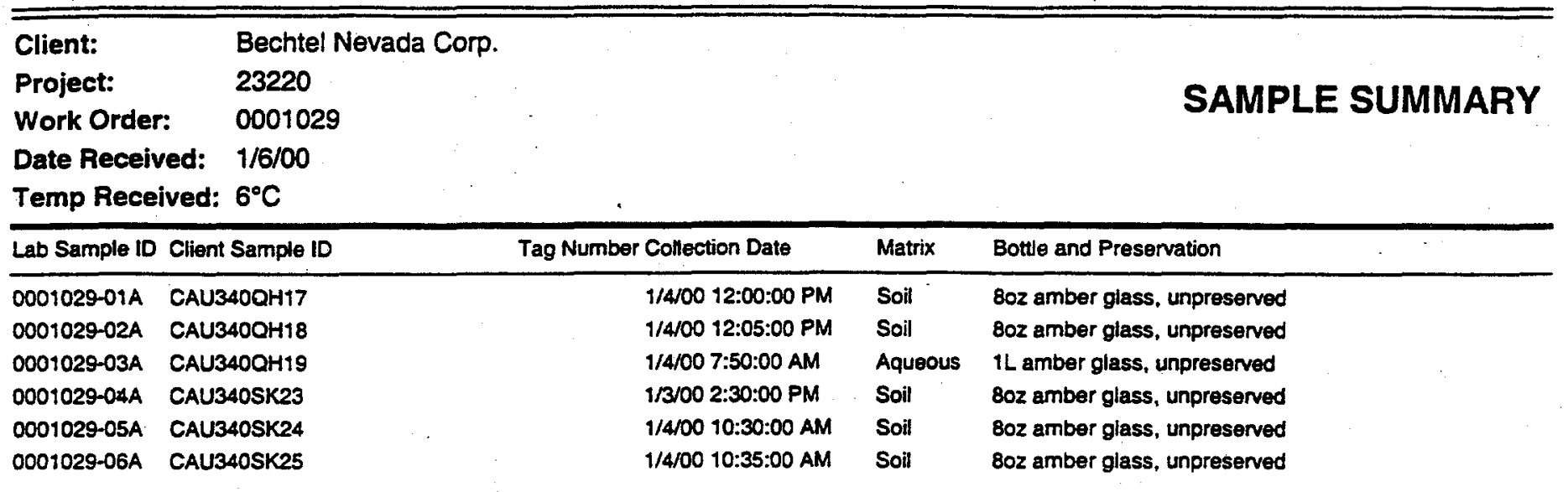


Date: 17-Jan-00

\section{Barringer Laboratories, Inc.}

15000 W 6th Avenue Suire 300 Golden, Colorado 80401.5047

(800) 654-0506

(303) $277-1687 \operatorname{Fax}(303) 277-1689$

Client: Bechtel Nevada Corp.

Project: 23220

Work Order: $\quad 0001029$

CASE NARRATIVE

All reported values in this report have been rounded to the correct number of significant figures. All calculations have been performed before applying significant figures, therefore, not all calculations may be reproducible with the results printed in this report. 
Date: 17-Jan-00 Barringer Laboratories, Inc.

CLIENT SAMPLE REPORT

15000 W 6th Avemue Suite 100 Golden, Collorado 80401-5047 (800) 6.54.0506 (30.3) 277.1687 Far (30.3) 277.1689

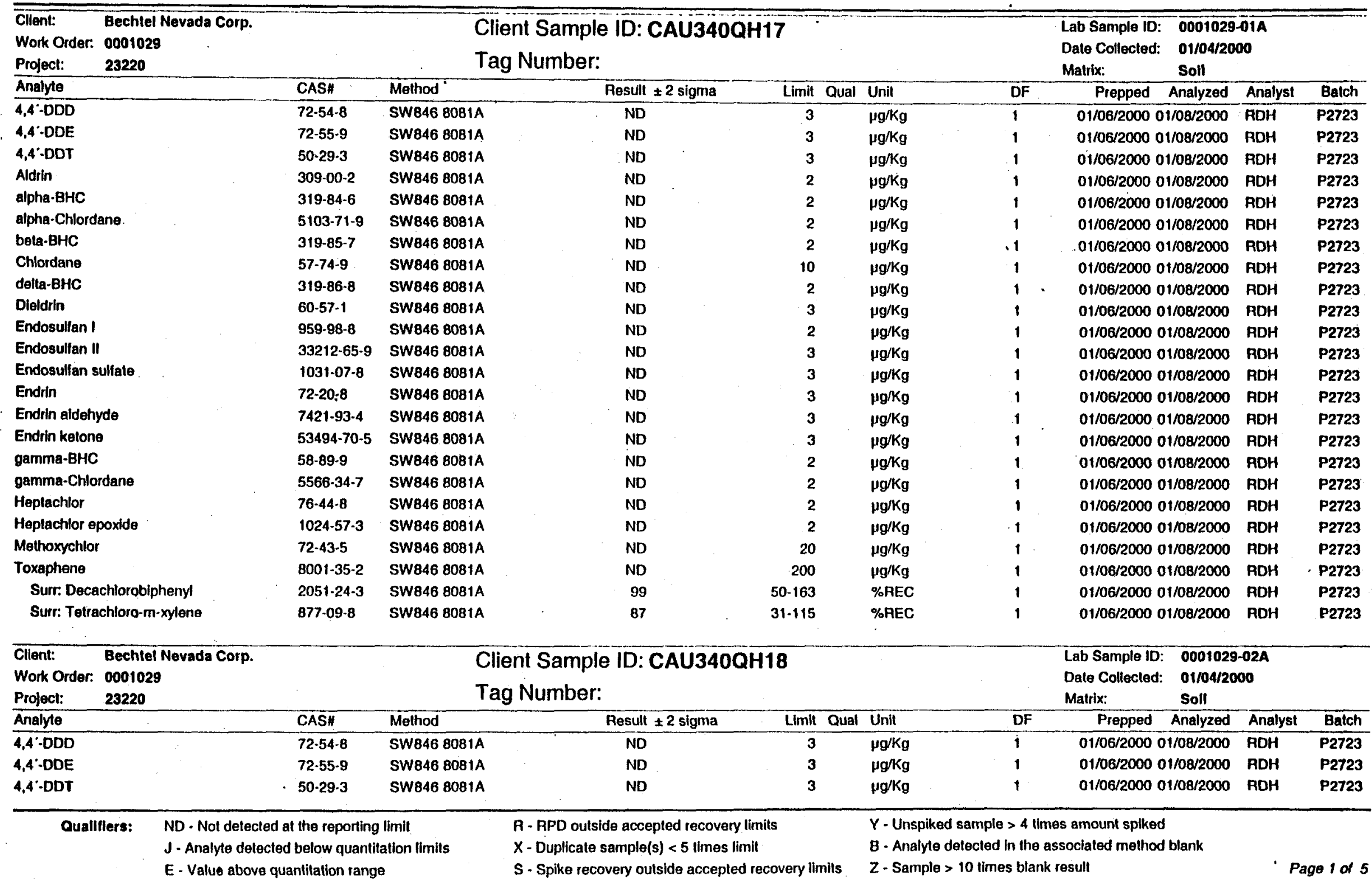


Date: 17-Jàn-00 Barringer Laboratories, Inc.

\section{CLIENT SAMPLE REPORT} 15000 W 6th Avenue Suite 300 Golden, Colorado 80401.5047 (800)654-0.506 (303) 277.1687 Fax (303) 277-1689

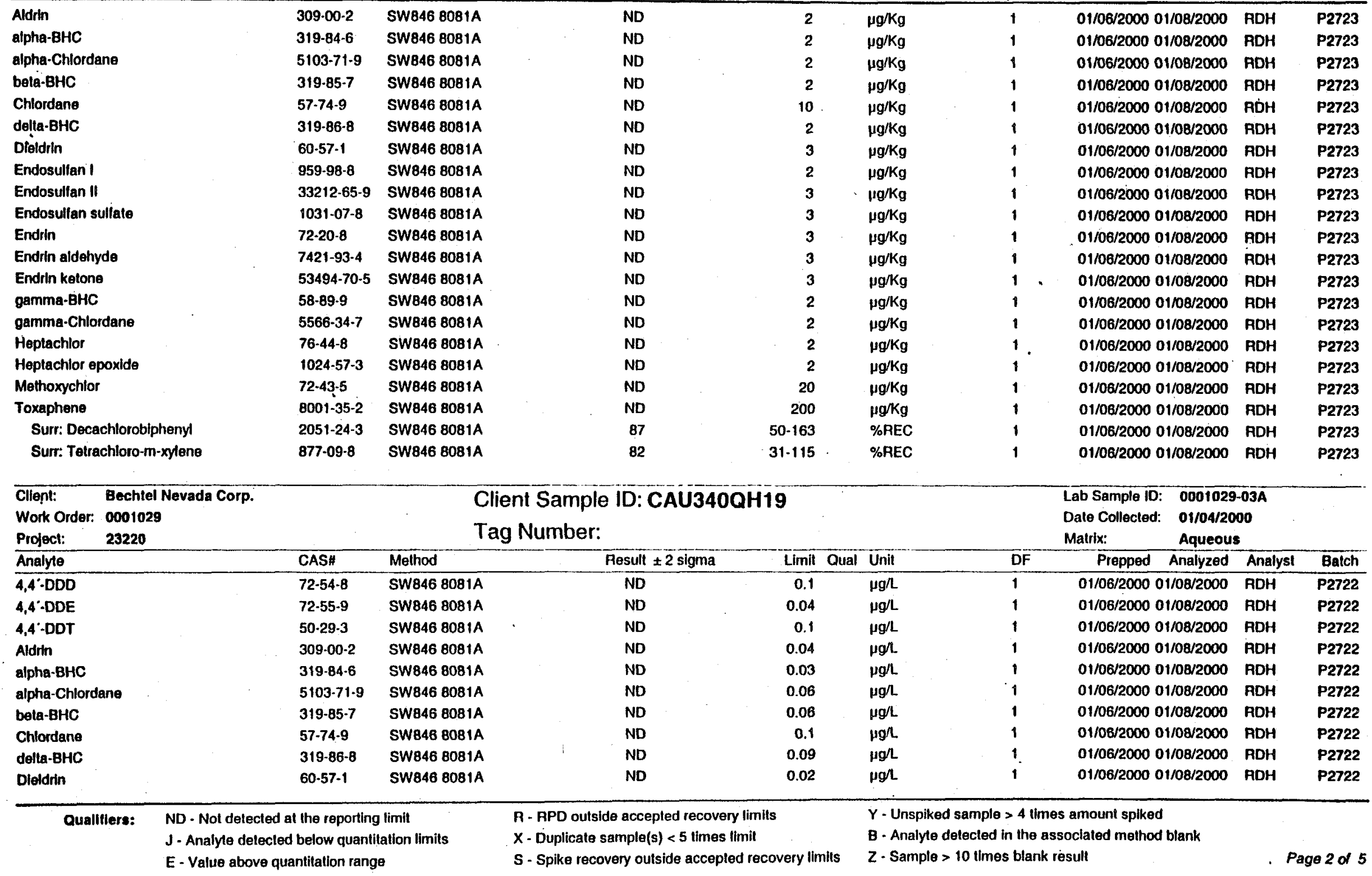


Date: 17-Jan-00 Barringer Laboratories, Inc.

CLIENT SAMPLE REPORT

15000 W6 6h Avemue Suite 300 Golden, Colorado 80401.5047 (800)6.54.0506 (30.3) 277.1687 Fax (303) 277.1689

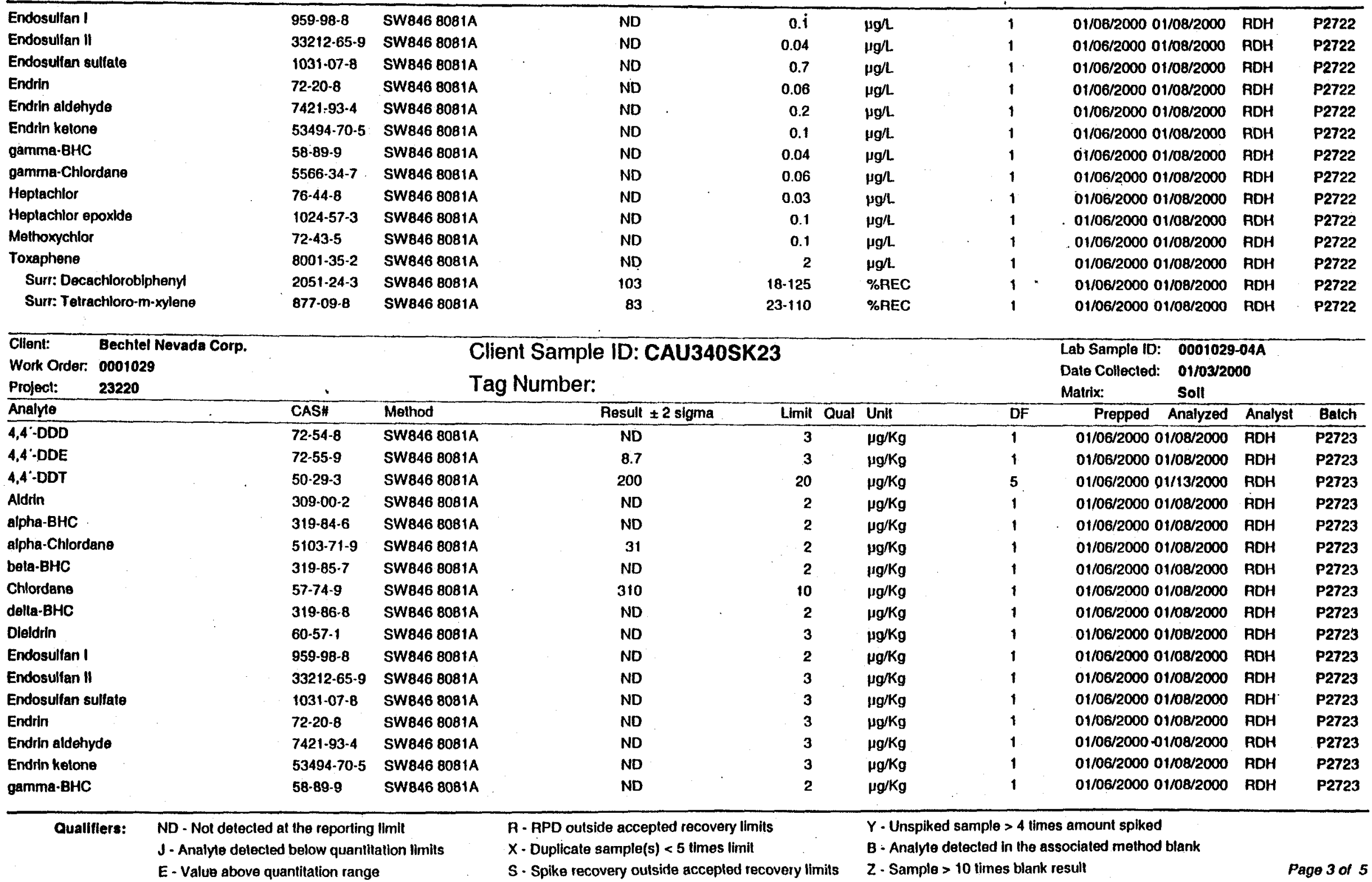


Date: 17-Jan-00 Barringer Laboratories, Inc.

CLIENT SAMPLE REPORT

15000 W 6ih Avemue Suire 300 Golden, Colorado 80401.5047

(800) 654-0506 (.303) 277-1687 $\operatorname{Fax}(303) 277.1689$

\begin{tabular}{llll}
\hline gamma-Chlordane & $5566-34-7$ & SW846 8081A & S3 \\
Heplachlor & $76-44-8$ & SW846 8081A & \\
Heplachlor epoxide & $1024-57-3$ & SW846 8081A & ND \\
Melhoxychlor & $72-43-5$ & SW846 8081A & ND \\
Toxaphene & $8001-35-2$ & SW846 8081A & 102 \\
$\quad$ Surr: Decachlorobiphenyl & $2051-24-3$ & SW846 8081A & 91 \\
Surr: Tetrachloro-m-xylene & $877-09-8$ & SW846 8081A &
\end{tabular}

$\begin{array}{rrrr}33 & 2 & \mu g / \mathrm{kg} & 1 \\ 15 & 2 & \mu g / \mathrm{Kg} & 1 \\ \text { ND } & 2 & \mu g / \mathrm{Kg} & 1 \\ \text { ND } & 20 & \mu g / \mathrm{Kg} & 1 \\ \text { ND } & 200 & \mu g / \mathrm{Kg} & 1 \\ 102 & 50-163 & \% \mathrm{REC} & 1 \\ 91 & 31-115 & \text { \%REC } & 1\end{array}$

01/06/2000 01/08/2000 RDH

P2723

$01 / 06 / 2000$ 01/08/2000 RDH

01/06/2000 01/08/2000 RDH

01/06/2000 01/08/2000 ADH

01/06/2000 01/08/2000 RDH

$01 / 06 / 2000$ 01/08/2000 RDH

$01 / 06 / 2000$ 01/08/2000 ADH

22723

P2723

P2723

P2723

P2723

P2723

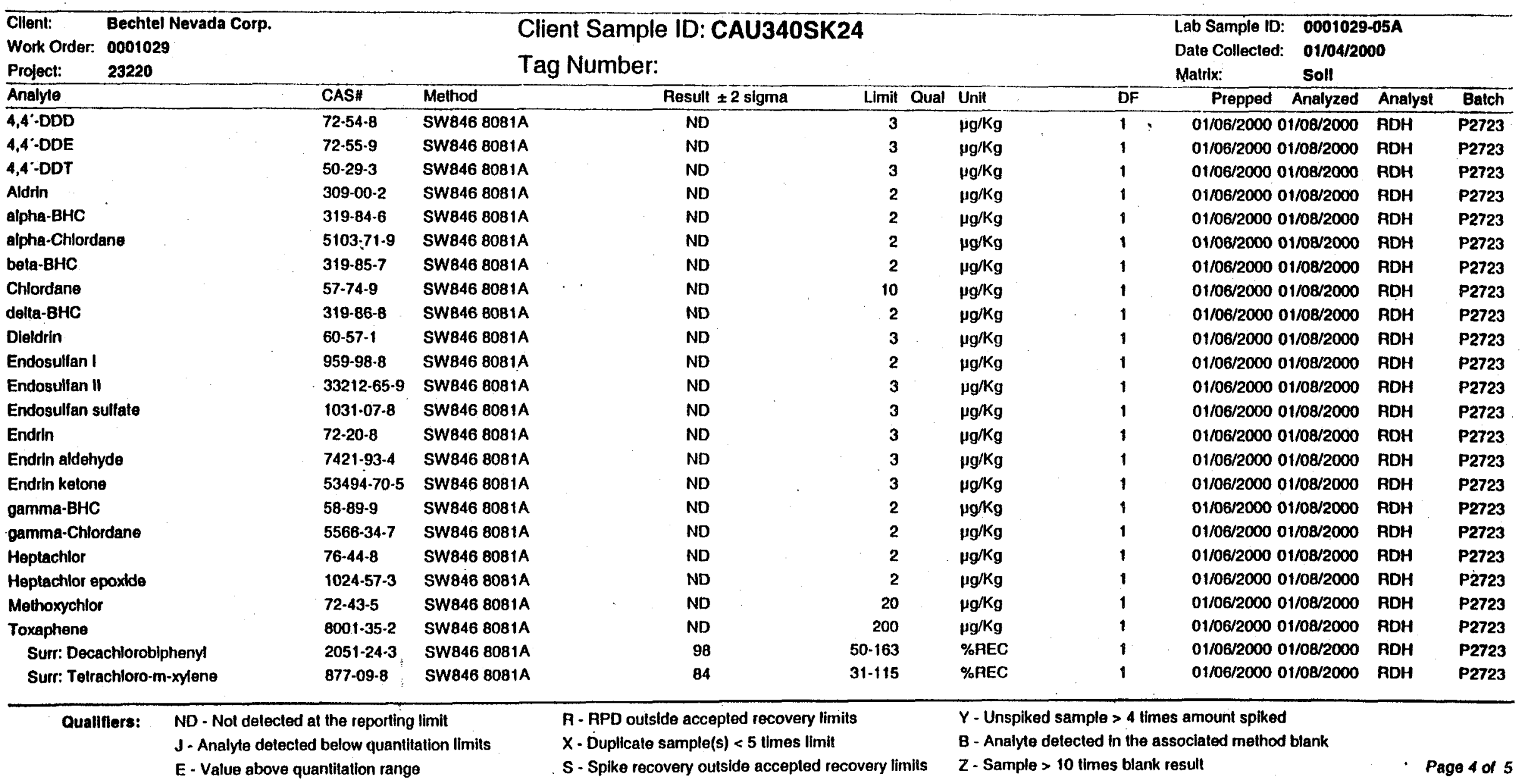


Date: 17-Jan-00 Barringer Laboratories, Inc.

CLIENT SAMPLE REPORT

15000 W Gih Avenue Suile 300 Golden, Colorado 80401.5047 (800) $6.54 .0506 \quad$ (303) 277.1687 Fax (303) 277.1689

\begin{tabular}{|c|c|c|c|c|c|c|c|c|c|c|c|}
\hline $\begin{array}{ll}\text { Cllent: } & \text { Bechtel Nevada Corp. } \\
\text { Work Order: } & 0001029 \\
\text { Project: } & 23220 \\
\end{array}$ & & & e ID: CAU34C & & & & & $\begin{array}{l}\text { Lab Sample ID: } \\
\text { Date Collected: } \\
\text { Malrix: }\end{array}$ & $\begin{array}{l}0001029 \\
01 / 04 / 20 \\
\text { Soll }\end{array}$ & & \\
\hline Analyte & CAS\# & Molhod & Result \pm 2 sigma & Limil & Qual & Unil & DF & Prepped & Analyzod & Analyst & Balch \\
\hline $4,4^{\circ} \cdot \mathrm{DDD}$ & $72 \cdot 54-8$ & SW846 8081A & ND & 3 & & $\mu g / \mathrm{kg}$ & 1 & $01 / 06 / 20000$ & $01 / 08 / 2000$ & RDH & P2723 \\
\hline 4,4'DDE & $72-55-9$ & SW846 8081A & ND & 3 & & $\mu g / K g$ & 1 & $01 / 06 / 20000$ & $01 / 08 / 2000$ & RDH & P2723 \\
\hline $4,4^{\circ}-\mathrm{DDT}$ & $50-29-3$ & SW846 8081A & ND & 3 & & $\mu g / K g$ & 1 & $01 / 06 / 2000$ & $01 / 08 / 2000$ & RDH & P2723 \\
\hline Aldrin & $309-00-2$ & SW846 8081A & ND & 2 & & $\mu g / K g$ & 1 & $01 / 06 / 2000$ & $01 / 08 / 2000$ & RDH & P2723 \\
\hline alpha-BHC & $319-84 \cdot 6$ & SW846 8081A & ND & 2 & & $\mu g / \mathrm{Kg}$ & 1 & $01 / 06 / 2000$ & $01 / 08 / 2000$ & RDH & P2723 \\
\hline alpha-Chlordane & $5103-71.9$ & SW846 8081A & ND & 2 & & $\mu g / \mathrm{Kg}$ & 1 & $01 / 06 / 2000$ & $01 / 08 / 2000$ & RDH & P2723 \\
\hline bela-BHC & $319-85-7$ & SW846 8081A & ND & 2 & & $\mu g / K g$ & 1 & $01 / 06 / 2000$ & $01 / 08 / 2000$ & RDH & P2723 \\
\hline Chlordane & $57-74-9$ & SW846 8081A & ND & 10 & & $\mu g / \mathrm{Kg}$ & $1:$ & $01 / 06 / 2000$ & 01/08/2000 & RDH & P2723 \\
\hline delta-BHC & $319-86-8$ & SW846 B081A & ND & 2 & & $\mu g / \mathrm{Kg}$ & 1 & $01 / 06 / 2000$ & $01 / 08 / 2000$ & RDH & P2723 \\
\hline Dleldrin & $60-57-1$ & SW846 B081A & ND & 3 & & $\mu g / \mathrm{Kg}$ & 1 & $01 / 06 / 2000$ & $01 / 08 / 2000$ & RDH & P2723 \\
\hline Endosullan I & 959-98-8 & SW846 8081A & ND & 2 & & $\mu g / K g$ & 1 & $01 / 06 / 2000$ & $01 / 08 / 2000$ & RDH & P2723 \\
\hline Endosullan II & $33212-65-9$ & SW846 B081A & ND & 3 & & $\mu g / K g$ & 1 & $01 / 06 / 2000$ & $01 / 08 / 2000$ & RDH & P2723 \\
\hline Endosullan sullate & $1031 ; 07-8$ & SW846 B081A & ND & 3 & & $\mu \mathrm{g} / \mathrm{Kg}$ & 1 & $01 / 06 / 2000$ & $01 / 08 / 2000$ & $\mathrm{ADH}$ & P2723 \\
\hline Endrin & $72-20-8$ & SW846 8081A & ND & 3 & & $\mu g / K g$ & 1 & $01 / 06 / 2000$ & $01 / 08 / 2000$ & RDH & P2723 \\
\hline Endrin aldehyde & $7421-93-4$ & SWB46 B081A & ND & 3 & & $\mu g / \mathrm{Kg}$ & 1 & $01 / 06 / 2000$ & $01 / 08 / 2000$ & RDH & P2723 \\
\hline Endrin ketone & $53494-70-5$ & SW846 8081A & ND & 3 & & $\mu g / K g$ & 1 & $01 / 06 / 2000$ & $01 / 08 / 2000$ & RDH & P2723 \\
\hline gamma-BHC & $58-89-9$ & SW846 8081A & ND & 2 & & $\mu g / \mathrm{Kg}$ & 1 & $01 / 06 / 2000$ & $01 / 08 / 2000$ & ADH & P2723 \\
\hline gamma-Chlordane & $5566-34-7$ & SW846 8081A & ND & 2 & & $\mu g / K g$ & 1 & $01 / 06 / 2000$ & $01 / 08 / 2000$ & ROH & P2723 \\
\hline Heptachlor & $76-44-8$ & SW846 8081A & ND & 2 & & $\mu g / K g$ & 1 & $01 / 06 / 2000$ & $01 / 08 / 2000$ & RDH & P2723 \\
\hline Heptachlor epoxide & $1024-57-3$ & SWB46 B081A & ND & 2 & & $\mu g / \mathrm{Kg}$ & 1 & $01 / 06 / 2000$ & $01 / 08 / 2000$ & RDH & P2723 \\
\hline Methoxychlor & $72-43-5$ & SW846 8081A & ND & 20 & & $\mu g / \mathrm{Kg}$ & 1 & $01 / 06 / 2000$ & $01 / 08 / 2000$ & RDH & P2723 \\
\hline Toxaphene & $8001 \cdot 35-2$ & SW846 B081A & ND & 200 & & $\mu g / \mathrm{Kg}$ & 1 & $01 / 06 / 2000$ & $01 / 08 / 2000$ & ADH & P2723 \\
\hline Surr: Decachlorobiphenyl & $2051 \cdot 24-3$ & SWB46 B081A & 106 & $50-163$ & & $\%$ REC & 1 & $01 / 06 / 2000$ & $01 / 08 / 2000$ & ADH & P2723 \\
\hline Surr: Tetrachloro-m-xylene & $877-09-8$ & SWB46 B081A & 95 & $31-115$ & & $\%$ REC & 1 & $01 / 06 / 2000$ & $01 / 08 / 2000$ & RDH & P2723 \\
\hline
\end{tabular}

$\begin{array}{ll}\text { Quallifers: } & \text { ND - Not delected at the reporting limit } \\ & \mathrm{J} \text { - Analyte detected below quantitation limits } \\ \mathrm{E}-\text { Value above quantilation range }\end{array}$
A - RPD outside accepled recovery limits

$X$ - Duplicate sample(s) $<5$ times limil

S - Spike recovery outside accepted recovery limits
$Y$ - Unspiked sample $>4$ times amount splked

B - Analyte detected in the assoclated method blank

Z. Sample > 10 times blank resull 

Date: 17-Jan-00 Barringer Laboratories, Inc.

BATCH QC SUMMARY REPORT

15000 W 6th Avenue Suile 300 Golden, Colorado 80401-5047 (800)654-0506 (30.3) 277-1687 Fax (303) 277-1689

\begin{tabular}{|c|c|c|c|c|c|c|c|c|c|c|c|c|c|}
\hline \multirow[t]{2}{*}{$\begin{array}{l}\text { Cllent: } \quad \text { Bechlel Nevada Corp. } \\
\text { Work Order: } 0001029 \\
\text { Prolect: } \quad 23220 \\
\end{array}$} & \multicolumn{3}{|c|}{$\begin{array}{l}\text { Batch ID: P2722 } \\
\text { Laboratory Control Spike }\end{array}$} & & $\begin{array}{l}\text { Seq No: } \\
\text { Run ID: }\end{array}$ & \multicolumn{3}{|c|}{$\begin{array}{l}76347 \\
\text { MOE_000107A }\end{array}$} & $\begin{array}{l}\text { Unit: } \\
\text { Malrix: }\end{array}$ & $\begin{array}{l}\mu g / L \\
\text { Aqueous }\end{array}$ & $\begin{array}{l}\text { Analyzed: } \\
\text { Analyst: } \\
\end{array}$ & \multicolumn{2}{|c|}{$\begin{array}{l}\text { Prepped: } 1 / 6 / 00 \\
\text { Analyzed: } 1 / 8 / 00 \\
\text { Analyst: } \mathrm{RDH}\end{array}$} \\
\hline & Result \pm 2 sigma & Limit & SpikeVal & SpikeRe & $\mathrm{Nal}$ & $\%$ REC & Limit Hig & hlimil & DupReIVal & \pm 2 sigma & APD/REA & APDLimit & Qual \\
\hline $4,4^{\circ} \cdot \mathrm{DDT}$ & $0 . \overline{84}$ & 0.1 & 1.0 & & & 84 & 33 & 143 & & & & & \\
\hline Aldrin & 0.31 & 0.04 & 0.40 & & & 77 & 1 & 136 & & & & & \\
\hline Dleldrin & 0.75 & 0.02 & 1.0 & & & 75 & 15 & 138 & & . & & & \\
\hline Endrin & 0.78 & 0.06 & 1.0 & & & 78 & 1 & 150 & & & & & \\
\hline gamma-BHC & 0.30 & 0.04 & 0.40 & & & 74 & 7 & 124 & & . & & & \\
\hline Heplachlor & 0.30 & 0.03 & . $\quad 0.40$ & & & 75 & 24 & 132 & & & & & . \\
\hline Surr: Decachloroblphenyl & & & . & & & 101 & 18 & 125 & & .. & & & \\
\hline Surr: Tetrachloro-m-xylene & & & & & & 85 & 23 & 110 & . & & & . & \\
\hline $\begin{array}{ll}\text { Client: } & \text { Bechtel Nevada Corp. } \\
\text { Work Order: } 0001029 \\
\text { Project: } \quad 23220 \\
\end{array}$ & $\begin{array}{l}\text { Batch ID: P } \\
\text { Laboratory }\end{array}$ & ol Sp & re Dupl & Icate & $\begin{array}{l}\text { Sample } \\
\text { Seq No: } \\
\text { Run ID: }\end{array}$ & 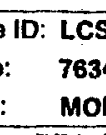 & 2722 & & $\begin{array}{l}\text { Method: } \\
\text { Unit: } \\
\text { Matrix: }\end{array}$ & $\begin{array}{l}\text { SW846 } 8081 A \\
\mu g h \text {. } \\
\text { Aqueous }\end{array}$ & & $\begin{array}{l}\text { Prepped: } \\
\text { Analyzed: } \\
\text { Analyst: }\end{array}$ & $\begin{array}{l}1 / 6 / 00 \\
1 / 8 / 00 \\
\text { RDH }\end{array}$ \\
\hline Analyte & Resull \pm 2 sigma & Limit & SpikeVal & Spike & afval & $\%$ REC & Limit Hi & hLlmil & DupRelval & \pm 2 sigma & RPD/AER & APOLIIII & Qual \\
\hline $4,4^{\circ} \cdot \mathrm{DOT}$ & .0 .96 & 0.1 & 1.0 & & & 96 & 33 & 143 & 0.84 & & 13 & 20 & 0 \\
\hline Aldrin & 0.33 & 0.04 & 0.40 & & & 82 & 1 & 136 & 0.31 & & 6 & 20 & 0 \\
\hline Dleldrin & 0.80 & 0.02 & 1.0 & & & 80 & 15 & 138 & 0.75 & & 7 & 20 & 0 \\
\hline Endrin & 0.84 & 0.06 & 1.0 & & & 84 & 1 & 150 & 0.78 & & 8 & 20 & 0 \\
\hline gamma-BHC & 0.32 & 0.04 & 0.40 & & & 79 & 7 & 124 & 0.30 & & 7 & 20 & 0 \\
\hline Heptachlor & 0.33 & 0.03 & 0.40 & & & 82 & 24 & 132 & 0.30 & & 8 & 20 & 0 \\
\hline Surr: Decachloroblphenyl & & & & & & 106 & 18 & 125 & & & & & \\
\hline Surr: Tetrachloro-m-xylene & & & & & & 89 & 23 & 110 & & & & & \\
\hline $\begin{array}{ll}\text { Cllent: } & \text { Bechtel Nevada Corp. } \\
\text { Work Order: } 0001029 \\
\text { Project: } \quad 23220 \\
\end{array}$ & $\begin{array}{l}\text { Batch ID: P } \\
\text { Method Ble }\end{array}$ & & & & $\begin{array}{l}\text { Sample } \\
\text { Seq No: } \\
\text { Run ID: }\end{array}$ & $\begin{array}{l}10: \overline{B B} \\
\quad 763 \\
M O t\end{array}$ & 2722 & & $\begin{array}{l}\text { Melhod: } \\
\text { Unit: } \\
\text { Matrix: }\end{array}$ & $\begin{array}{l}\text { SW846 8081A } \\
\mu g / \text {. } \\
\text { Aqueous }\end{array}$ & & $\begin{array}{l}\text { Prepped: } \\
\text { Analyzed: } \\
\text { Analyst: }\end{array}$ & $\begin{array}{l}1 / 6 / 00 \\
1 / 8 / 00 \\
\text { ADH }\end{array}$ \\
\hline Analyte & Resull \pm 2 sigma & Limit & SpikeVal & SpikeRe & $3 \mathrm{Nal}$ & \%REC I & Limil Hi & hLimit & DupRefval & \pm 2 sigma & RPD/AER & APDLimit & ilt Qual \\
\hline $4,4-D D D$ & ND & 0.1 & & & & & & & & & & & \\
\hline $4,4^{\prime \prime}$-DDE & ND & 0.04 & & & & & & & & & & & \\
\hline $4,4^{\circ}-D D T$ & ND & 0.1 & & & & & & & & . & & & \\
\hline Aldrin & ND & 0.04 & & & & & & & & & & & \\
\hline alpha-BHC & ND & 0.03 & & & & & & & & & & & \\
\hline alphà.Chlordane & ND & 0.06 & & & & & & & . & & & & \\
\hline
\end{tabular}

Quallfiers: ND - Nol delected at the reporting limit

$J$ - Analyte delecled below quantitation limits

$E \cdot$ Value above quantitation range
R - APD outside Bccepted recovery limits

$X$ - Duplicate sample(s) < 5 limes limil

S - Spike recovery outside accepted recovery limits
$Y$ - Unspiked sample > 4 limes amount spiked

B - Analyte detected in the associated method blank

$Z$ - Sample $>10$ times blank result 
Date: 17-Jan-00

Barringer Laboratories, Inc.

BATCH QC SUMMARY REPORT

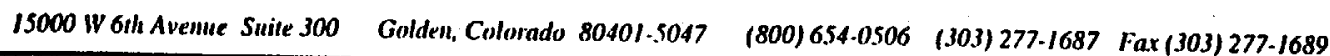

\begin{tabular}{llr}
\hline beta-BHC & ND & 0.06 \\
Chlordane & ND & 0.1 \\
delta-BHC & ND & 0.09 \\
Dieldrin & ND & 0.02 \\
Endosullan I & ND & 0.1 \\
Endosullan II & ND & 0.04 \\
Endosullan sulfale & ND & 0.7 \\
Endrin & ND & 0.06 \\
Endrin aldehyde & ND & 0.2 \\
Endrin kelone & ND & 0.1 \\
gamma-BHC & ND & 0.04 \\
gamma-Chlordane & ND & 0.06 \\
Heplachlor & ND & 0.03 \\
Heptactilor epoxlde & ND & 0.1 \\
Methoxychlor & ND & 0.1 \\
Toxaphene & ND & 2 \\
Surr: Decechlorobipheny & &
\end{tabular}

Surr: Decachiorobiphenyl

Surr: Tetrachloro-m-xylene

$105 \quad 18 \quad 125$

$91 \quad 23 \quad 110$

Client: Bechtel Nevada Corp.

Work Order, 0001029

Project: 20220

Batch ID: P2723

Analyte Laboratory Control Spike

Sample 1D: LCS-2723

Seq No: 76351

Run ID: MOE_000107B

Method: SW $8468081 A$

MOE_000107B Matrix: Soil

Prepped: 1/6/00

Analyzed: 1/8/00

Analysi: ROH

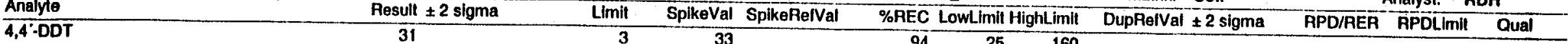

Aldrin

Dleldrin

Endrin

gamma-BHC

Heptachior

27

Surr: Decachiorobiphenyl

9.7

$\begin{array}{ll}3 & 33 \\ 2 & 13\end{array}$

$25 \quad 160$

Surr: Tetrachloro-m-xylene

$\begin{array}{rrr}78 & 42 & 122 \\ 76 & 36 & 146 \\ 81 & 30 & 147 \\ 79 & 32 & 127 \\ 73 & 34 & 111 \\ 107 & 50 & 163 \\ 82 & 31 & 115\end{array}$

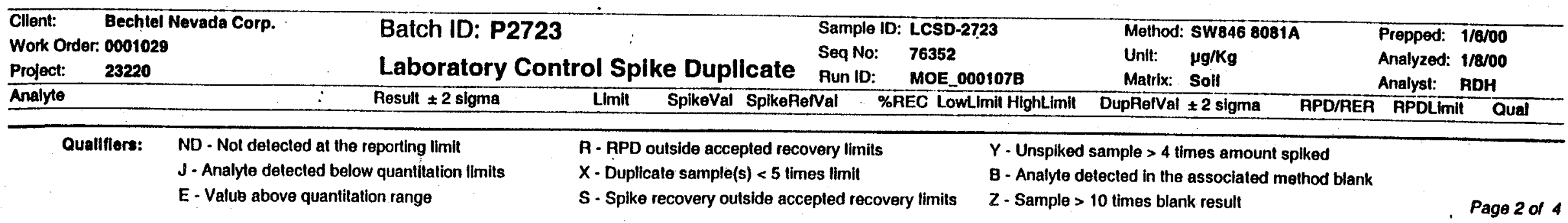


-

\section{BATCH QC SUMMARY REPORT}

\begin{tabular}{|c|c|c|c|}
\hline 1 & $15000 \mathrm{~W}$ 6th Avemue Suite 300 & Golden, Colorndo 80401-5047 & $(800) 6.54-0506$ \\
\hline $4,4^{\prime} \cdot$ DDT & & 29 & 3 \\
\hline Aldrin & & 9.8 & 2 \\
\hline Dleidrin & & 24 & 3 \\
\hline Endrin & & 25 & 3 \\
\hline gamma-вHC & & 9.8 & 2 \\
\hline $\begin{array}{l}\text { Heptachlor } \\
\text { Surr: Decac }\end{array}$ & chlorobiphenyl & 9.6 & 2 \\
\hline
\end{tabular}

Surr: Decachlorobiphenyl

$\begin{array}{lrllllll}33 & 87 & 25 & 160 & 31 & 8 & 20 \\ 13 & 74 & 42 & 122 & 10 & 6 & 20 \\ 33 & 72 & 36 & 146 & 25 & 6 & 20 \\ 33 & 76 & 30 & 147 & 27 & 6 & 20 \\ 13 & 73 & 32 & 127 & 10 & 7 & 20 \\ 13 & 72 & 34 & 111 & 9.7 & 2 & 20 \\ & 101 & 50 & 163 & & & \end{array}$

\section{Client: Bechlel Nevada Corp.}

Work Order: 0001029

Project: 23220

Analyte

23220

Batch ID: P2723

4,4'DDD

4,4:DOE

4.4'-DDT

Aldrin

alpha-BHC

alpha-Chlordane

bela-BHC

Chlordane

delta-BHC

Dieldrin

Endosulian I

Endosultan II

Endosulfan sulfate

Endrin

Endrin aldehydo

Endin kelone

gamma-BHC

gamma-Chlordane

Heptactilor

Heplachlor epoxide

Methoxychlor

Toxaphene

Method Blank

3

Fesult \pm 2 sigma

Result
ND
ND

ND

ND

ND

ND

ND.

ND

ND

ND

ND

ND

ND

ND

ND

ND

ND

ND

ND

ND

ND

ND

ND

31

115

Surr: Decachloroblphenyl

Quallfiers:
ND - Not delecled at the reporting limit
J - Analyte detected below quantitation limits
E. Value above quanlitation range

R - RPD outside accepted recovery limits

110

50

163

$X$ - Duplicate sample(s) $<5$ times limil

$Y$ - Unsplked sample > 4 limes amount spiked

S - Spike recovery outside accepted recovery limils

$B$ - Analyle detected in the assoclated melhod blank

Prepped: 1/6/00

Analyzed: 1/8/00

Analyst: RDH

Matrix: Soll

RPD/RER APDLimil

Qual


Date: 17-Jan-00 Barringer Laboratories, Inc.

BATCH QC SUMMARY REPORT

I5000 W 6th Avenue Suile 300 Goldell, Colorado 80401-5047 (800) 654-0506 (303) 277-1687 Fax (303) 277.1689

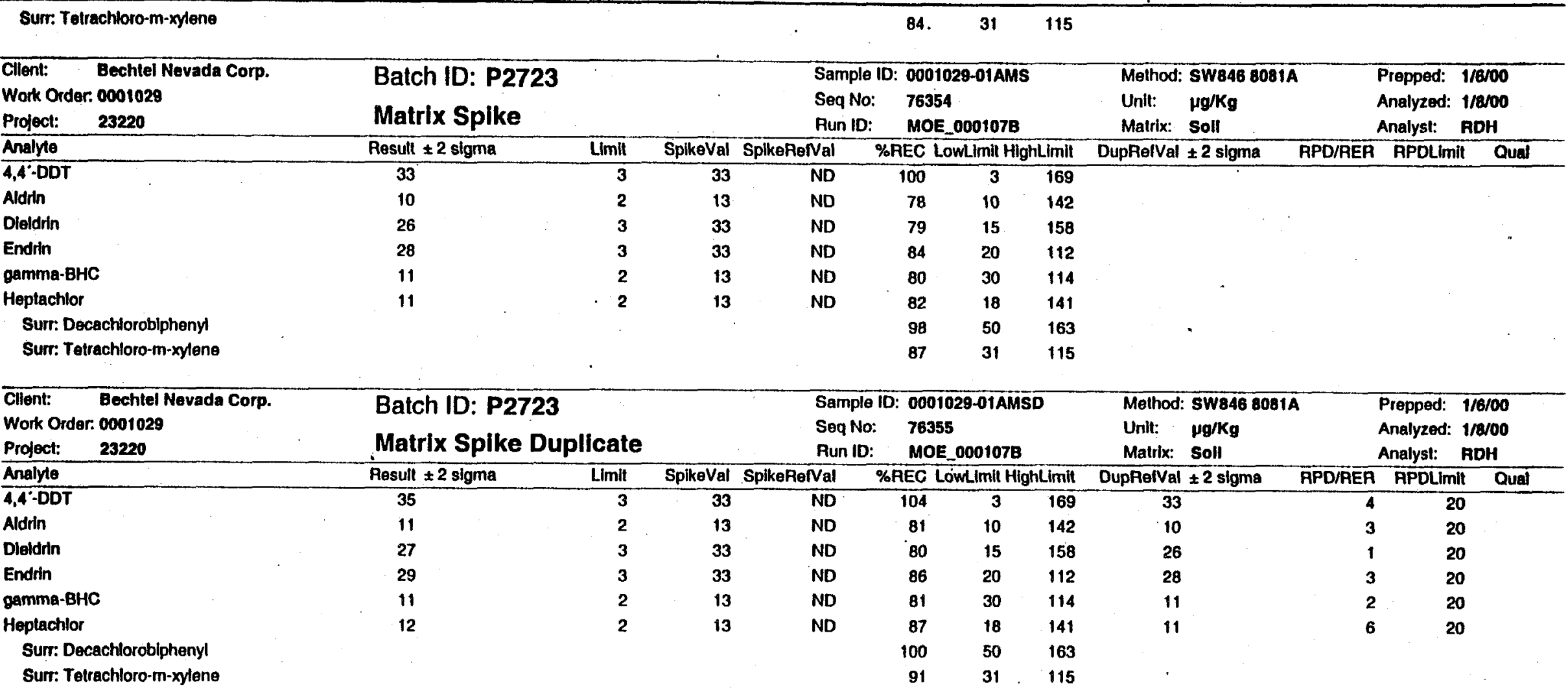

\footnotetext{
Qualifiers: ND - Not detected at the reporting limit

$J$ - Analyte delected below quantitation limits

E - Value above quantitallon range
}

R - APD outside accepted recovery limits

$Y$ - Unspiked sample $>4$ times amount spiked

$X$ - Duplicate sample(s) $<5$ times limit

$B$ - Analyte detected in the assoclaled method blank

S - Splke recovery outside accepted recovery limils $Z$ - Sample $>10$ times blank result 


\section{NTS - PRODUCTION}

CHAIN OF CUSTODY RECORD: CAU 340-6

Cooler Temp:

Number of Coolers:

Cooler Units:

Logbook No.: .

$$
\text { Facllity ID: NTS }
$$

Sampling Event: cav 340

COC Number: cAU 340-6
30G 据 V744

Delivery Order ID:

wi1786

SEIR No.: CAU 340-5-0

Laboratory: BARINGR

Print

Sign

Requested Analysis:

\begin{tabular}{|c|c|c|c|c|c|c|c|c|}
\hline Site ID & Station ID & Sample ID & $\begin{array}{l}\text { Samp } \\
\text { Type }\end{array}$ & $\begin{array}{l}\text { Matrlx } \\
\text { Code }\end{array}$ & $\begin{array}{l}\text { Collection } \\
\text { Start Date }\end{array}$ & StartTIme & $\begin{array}{l}\text { Collection } \\
\text { End Date }\end{array}$ & End Time \\
\hline \multirow[t]{2}{*}{23} & CAU 340 & CAU340QH17 & GRAB & so & $i-4-2000$ & 12:00 & $1-4-2000$ & $12: 00$ \\
\hline & Pay Item & Description & & & & $\begin{array}{l}\text { Parametor } \\
\text { Code }\end{array}$ & $\begin{array}{l}\text { Fill Prior } \\
\text { Cd Lvl }\end{array}$ & $\begin{array}{l}\text { Añal } \\
\text { Lvi }\end{array}$ \\
\hline
\end{tabular}

\begin{tabular}{lllllllll}
\hline 23 & CAU 340 CAU340QH18 & GRAB & So & $1-4-2000$ & $12: 05$ & $1-4-20200$ & $12: 05$
\end{tabular}

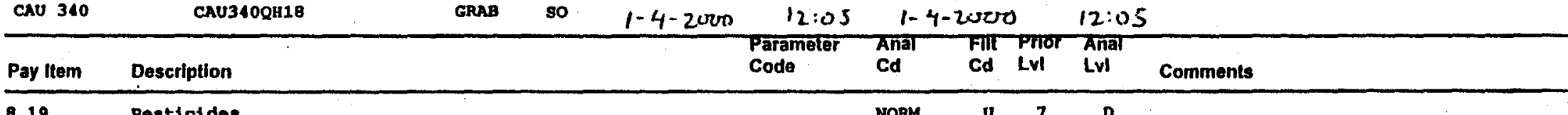

\section{3}

\begin{tabular}{|c|c|c|c|c|c|c|c|c|c|c|}
\hline Cav 340 & CAU340QH19 & EB & $\omega$ & $1-4-2000$ & $07: 50$ & & $4-20700$ & & $07: 50$ & \\
\hline Pay Item & Description & & & & $\begin{array}{l}\text { Parameter } \\
\text { Code }\end{array}$ & $\begin{array}{l}\text { Anat } \\
\text { Cd }\end{array}$ & $\begin{array}{l}\text { FilI } \\
\text { Cd }\end{array}$ & $\begin{array}{l}\text { Prid } \\
\text { LvI }\end{array}$ & $\begin{array}{l}\text { Añal } \\
\text { LvI }\end{array}$ & Comment \\
\hline 3.4 & Organochlorine Pesticides & & & & & REG & v & 7 & D & \\
\hline
\end{tabular}

This package conforms to the conditions and limilations specified in 49 CFR 173.421 for excepled radioactive malerial, llimited quanlity, n.0.s., UN2910. 
Requested Analysis:

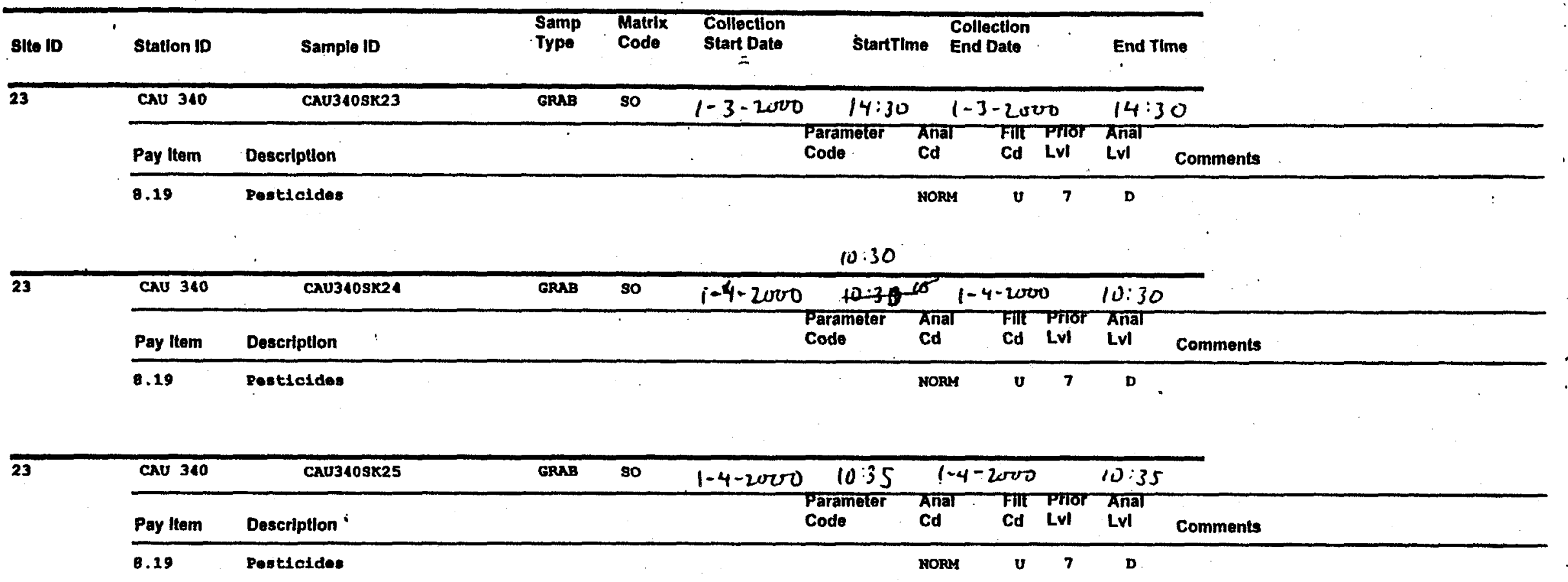


Containers Included on C.o.C.:

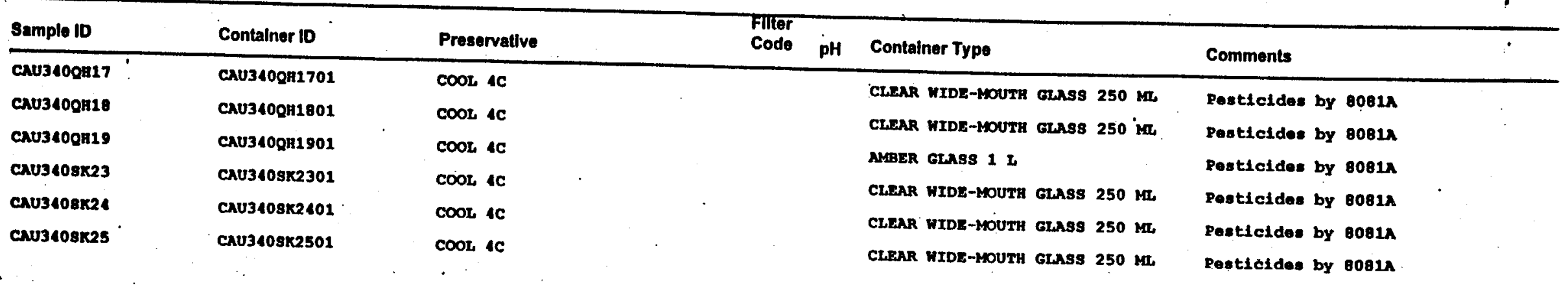




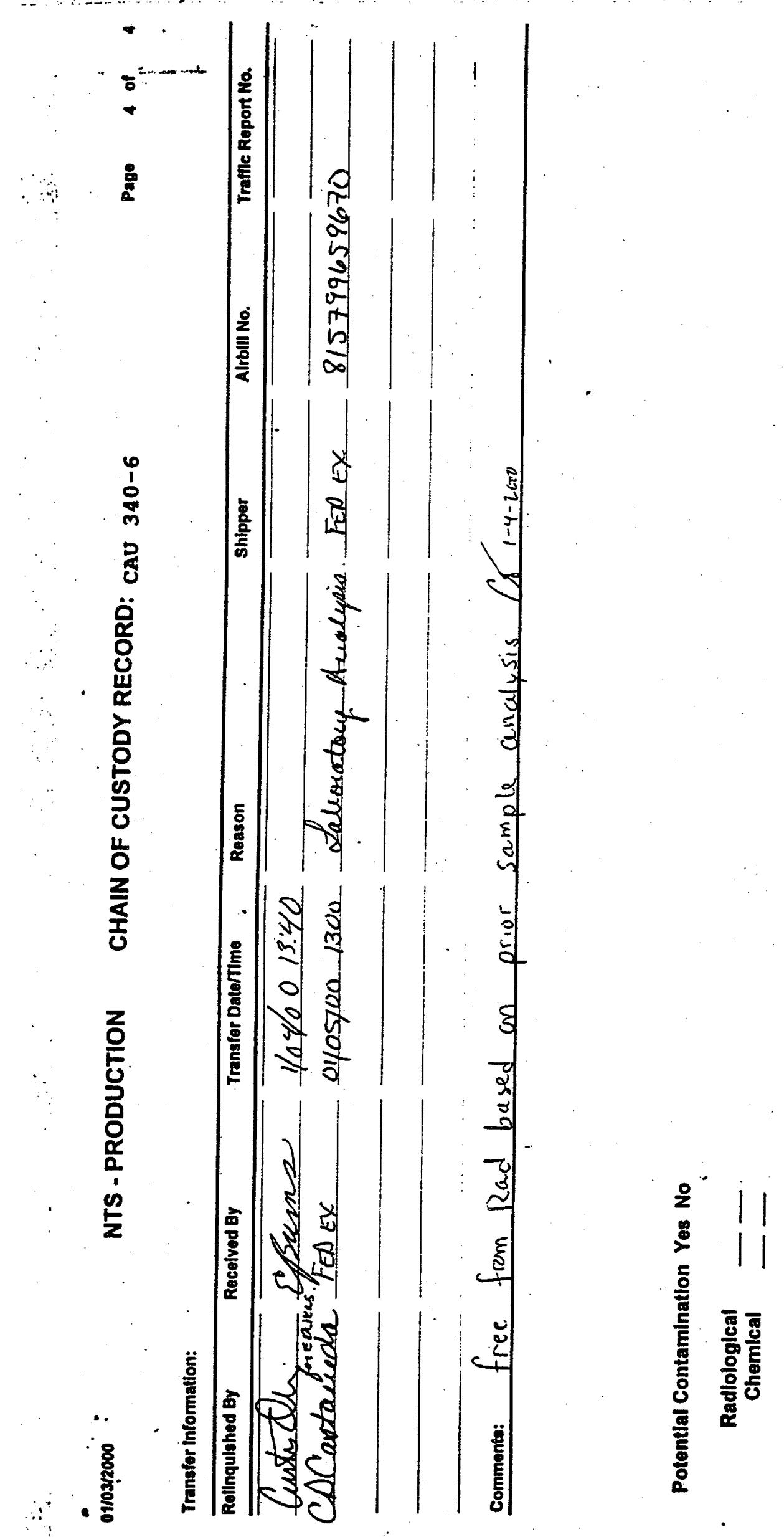

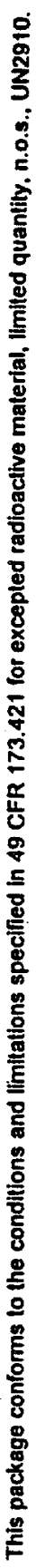




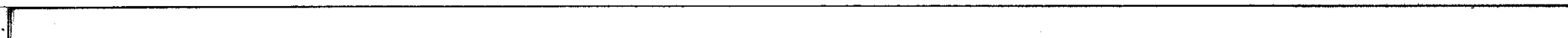

돌

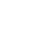




\section{DISTRIBUTION LIST}




.




\section{DISTRIBUTION LIST}

* Provide copy of initial distribution or Revision 0; remainder of list gets Revision 0 if approved without changes. The entire list receives Recision 1 , if issued.

\section{Nevada Division of Environmental Protection}

Paul Liebendorfer

2 (Controlled)*

Bureau of Federal Facilities

Division of Environmental Protection

333 W. Nye Lane, Room 13B

Carson City, NV 89706-0866

Mike McKinnon

1 (Controlled)*

Bureau of Federal Facilities

Division of Environmental Protection

Sawyer State Building

555 E. Washington, Suite 4300

Las Vegas, NV 89101-1049

\section{U.S. Department of Energy}

Janet Appenzeller-Wing

1 (Uncontrolled)*

Environmental Restoration Division

U.S. Department of Energy, Nevada Operations Office

P.O. Box $98518 \mathrm{M} / \mathrm{S} 505$

Las Vegas, NV 89193-8518

Clayton Barrow

1 (Uncontrolled)*

Environmental Restoration Division

U.S. Department of Energy, Nevada Operations Office

P.O. Box $98518 \mathrm{M} / \mathrm{S} 505$

Las Vegas, NV 89193-8518

Sabrina Lawrence

1 (Controlled)*

Environmental Restoration Division

U.S. Department of Energy, Nevada Operations Office

P.O. Box $98518 \mathrm{M} / \mathrm{S} 505$

Las Vegas, NV 89193-8518

U.S. Department of Energy

1 (Uncontrolled,

Office of Scientific and Technical Information electronic copy)

P.O. Box 62

Oak Ridge, TN 37831-0062 


\section{DISTRIBUTION LIST (continued)}

\section{U.S. Department of Energy (continued)}

U.S. Department of Energy

1 (Uncontrolled)

Nevada Operations Office

Technical Information Resource Center

P.O. Box 98518 , M/S 505

Las Vegas, NV 89193-8518

Manager, Southern Nevada

1 (Controlled) \&

FFACO Public Reading Room

1 (Uncontrolled)

P.O. Box 98521 , M/S NLV040

Las Vegas, NV 89193-8521

Manager, Northern Nevada

1 (Uncontrolled)

FFACO Public Reading Room

Nevada State Library and Archives Federal Publications

100 North Stewart Street

Carson City, NV 89701-4285

\section{Bechtel Nevada}

Correspondence Control

1 (Uncontrolled)*

Bechtel Nevada

P.O. Box 98521 M/S NLV008

Las Vegas, NV 89193-8521

Environmental Management Library

1 (Uncontrolled) *

Bechtel Nevada

P.O. Box 98521, M/S NLV080

Las Vegas, NV 89193-8521

David Cowser

1 (Uncontrolled)*

Bechtel Nevada

P.O. Box 98521 M/S NLV082

Las Vegas, NV 89193-8521

Ann Heidema

1 (Uncontrolled) *

Bechtel Nevada

P.O. Box 98521, M/S NLV022

Las Vegas, NV 89193-8521 


\section{DISTRIBUTION LIST (continued)}

\section{Bechtel Nevada (continued)}

Wayne Johnson

1 (Uncontrolled)*

Bechtel Nevada

P.O. Box 98521, M/S NTS306

Las Vegas, NV 89193-8521

Steve Nacht

1 (Uncontrolled)*

Bechtel Nevada

P.O. Box $98521 \mathrm{M} / \mathrm{S}$ NTS306

Las Vegas, NV 89193-8521

Shannon Parsons-DePry

1 (Uncontrolled)*

Bechtel Nevada

P.O. Box 98521 M/S NTS306

Las Vegas, NV 89193-8521

Curtis Obi

1 (Uncontrolled)*

Bechtel Nevada

P.O. Box 98521 M/S NTS306

Las Vegas, NV 89193-8521

\section{IT Corporation}

Brad Jackson

2 (Uncontrolled) *

IT Corporation

P.O. Box 93838, M/S NLV439

Las Vegas, NV 89193-3838

IT FFACO Support Office

1 (Controlled)

IT Corporation

P.O. Box 93838, M/S NLV439

Ĺas Vegas, NV 89193-3838 



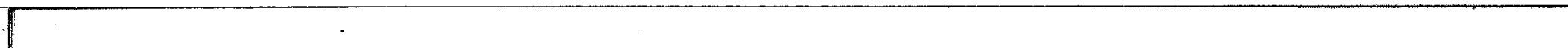


NEVADA DIVISION OF ENVIRONMENTAL PROTECTION DOCUMENT REVIEW SHEET 


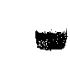




\section{DOCUMENT REVIEW SHEET}

Document Title/Number: Draft Closure Report for Corrective Action Unit 340: NTS Pesticide Release Sites

Document Date: March 2000

Revision Number: DRAFT

Originator/Organization: Bechtel Nevada /Environmental Restoration

Date Comments Due: March 31, 2000

Reviewer/Organization: NDEP (letter dated May 10, 2000, from Michael D. McKinnon to Runore C. Wycoff)

\begin{tabular}{||c|c|l|l|c||}
\hline $\begin{array}{c}\text { Comment } \\
\text { Number/ } \\
\text { Location }\end{array}$ & Type & Comment & Comment Response & Accept \\
\hline \hline 1 & $\mathrm{M}$ & $\begin{array}{l}\text { Since this CAU was remediated to } \\
\text { Industrial PRG standards, a land-use } \\
\text { restriction (LUR) must be imposed } \\
\text { and registered which restricts future } \\
\text { use of this site to industrial uses. }\end{array}$ & $\begin{array}{l}\text { Wording has been added to text in } \\
\text { Section 1 to reiterate that FFACO } \\
\text { sites are remediated to industrial } \\
\text { standards and future uses are } \\
\text { restricted to industrial uses. } \\
\text { Therefore, formal land-use } \\
\text { restrictions are not necessary. }\end{array}$ & No \\
\hline
\end{tabular}


